PRRC 04-21

\title{
IMPROVING $\mathrm{CO}_{2}$ EFFICIENCY FOR RECOVERING OIL IN HETEROGENEOUS RESERVOIRS
}

\author{
DOE CONTRACT NO. DE-FG26-01BC15364
}

New Mexico Petroleum Recovery Research Center

New Mexico Institute of Mining and Technology

801 Leroy Place

Socorro, NM 87801

(505) $835-5142$

Report Date: October 31, 2004

Contract Date: September 28, 2001

Completion Date: September 27, 2005

DOE Award of Third year: $\$ 337,000$

Program Manager: Reid B. Grigg

Principal Investigator: Reid B. Grigg

Other Major Contributors: Robert K. Svec

Zhengwen Zeng

Baojun Bai

Yi Liu

Contracting Officer's Representative: Paul West

Reporting Period: $\quad$ Oct. 1, 2003-Sept. 30, 2004 


\section{Disclaimer}

This report was prepared as an account of work sponsored by an agency of the United States Government. Neither the United States Government nor any agency thereof, nor any of their employees, makes any warranty, express or implied, or assumes any legal liability or responsibility for the accuracy, completeness, or usefulness of any information, apparatus, product, or process disclosed, or represents that its use would not infringe privately owned rights. Reference herein to any specific commercial product, process, or service by trade name, trademark, manufacturer, or otherwise does not necessarily constitute or imply its endorsement, recommendation, or favoring by the United States Government or any agency thereof. The views and opinions of authors expressed herein do not necessarily state or reflect those of the United States Government or any agency thereof. 


\section{Abstract}

The third annual report of "Improving $\mathrm{CO}_{2}$ Efficiency for Recovery Oil in Heterogeneous Reservoirs" presents results of laboratory studies with related analytical models for improved oil recovery. All studies were designed to optimize utilization and extend the practice of $\mathrm{CO}_{2}$ flooding to a wider range of reservoirs.

Chapter 1 describes the behavior at low concentrations of the surfactant Chaser International CD1045 ${ }^{\mathrm{TM}}$ (CD) versus different salinity, pressure and temperature. Results of studies on the effects of $\mathrm{pH}$ and polymer (hydrolyzed polyacrylamide-HPAM) and $\mathrm{CO}_{2}$ foam stability after adsorption in the core are also reported. Calcium lignosulfonate (CLS) transport mechanisms through sandstone, description of the adsorption of CD and CD/CLS onto three porous media (sandstone, limestone and dolomite) and five minerals, and the effect of adsorption on foam stability are also reported.

In Chapter 2, the adsorption kinetics of CLS in porous Berea sandstone and non-porous minerals are compared by monitoring adsorption density change with time. Results show that adsorption requires a much longer time for the porous versus non-porous medium. CLS adsorption onto sandstone can be divided into three regions: adsorption controlled by dispersion, adsorption controlled by diffusion and adsorption equilibrium. $\mathrm{NaI}$ tracer used to characterize the sandstone had similar trends to earlier results for the CLS desorption process, suggesting a dual porosity model to simulate flow through Berea sandstone.

The kinetics and equilibrium test for $\mathrm{CD}$ adsorption onto five non-porous minerals and three porous media are reported in Chapter 3. CD adsorption and desorption onto non-porous minerals can be established in less than one hour with adsorption densities ranging from 0.4 to $1.2 \mathrm{mg}$ of CD per $\mathrm{g}$ of mineral in decreasing order of montmorillonite, dolomite, kaolinite, silica and calcite. The surfactant adsorption onto three porous media takes much longer than one hour, with Berea sandstone requiring the longest time.

In Chapter 4, comparisons of static adsorption of CLS, CD, and CLS/CD mixtures onto five pure minerals showed that the presence of CLS decreased the adsorption of CD onto the five minerals by 20 to $70 \%$. Dynamic CLS/CD mixture adsorption tests onto Berea sandstone and Indian limestone cores showed that competitive adsorption between CD and CLS generally takes several days to reach equilibrium. Foam stability and interfacial tension tests on both injected and effluent samples were performed which showed that both foam stability and IFT decreased due to adsorption. Also it appears that there is a chromatographic effect on the surfactants in flow through porous media. Progress was realized in developing general equations for stress sensitivity on non-Darcy parameters (permeability and non-Darcy coefficient), and the multiphase flow induced by a high flow rate was confirmed as a mechanism for injectivity loss in $\mathrm{CO}_{2}$ flooding.

In Chapter 5, a general equation is defined based on 60 general equations of permeability stress sensitivity and non-Darcy coefficient stress sensitivity and definitions of nominal permeability, nominal non-Darcy coefficient, permeability stress sensitivity, and non-Darcy coefficient stress sensitivity. The equations of stress sensitivity are independent of pressure, temperature, and rock properties and existing empirical correlations of the nominal permeability and nominal non-Darcy coefficient can be used when laboratory data are not available. This provides a tool to quantify the change of permeability and non-Darcy coefficient due to change of effective stress resulted from reservoir injection and/or production. 
Results presented in Chapter 6 compare the high rate flow behavior of $\mathrm{N}_{2}$ and $\mathrm{CO}_{2}$ and show that the permeability and non-Darcy coefficient are different under similar experimental conditions. Under normal reservoir conditions, $\mathrm{N}_{2}$ does not deviate significantly from an ideal gas while $\mathrm{CO}_{2}$ might be gas like, liquid like, described as supercritical fluid, or transforming from one state to another. This phenomenon creates unstable regions and is difficult to predict results, including additional phases, rapid pressure changes, and localized reservoir temperature reduction. Each condition can result in increased pressure gradients, reduced injectivity and productivity, and formation damage. 


\section{TABLE OF CONTENTS}

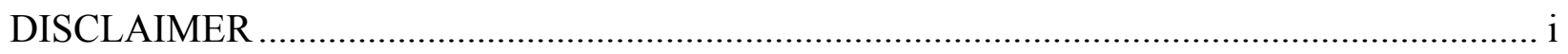

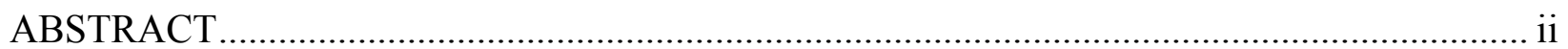

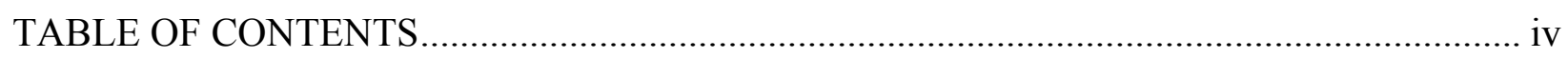

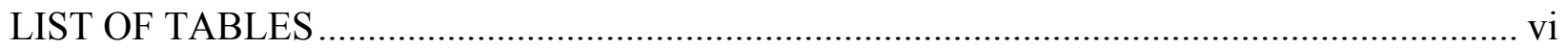

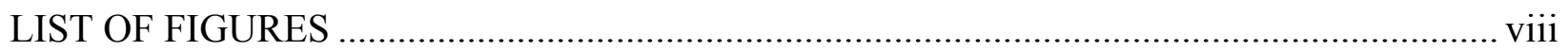

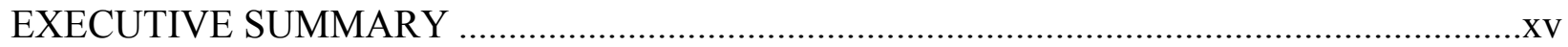

CHAPTER 1. INTERFACIAL TENSION AND CO2 FOAM STABILITY ............................. 1-1

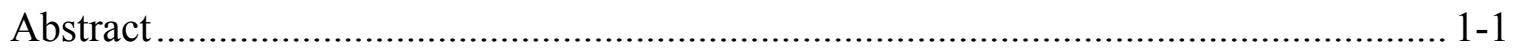

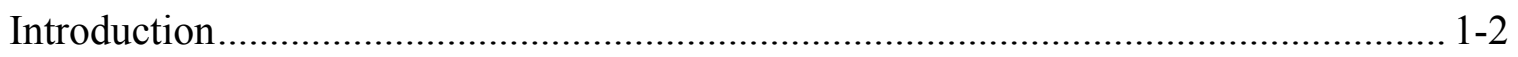

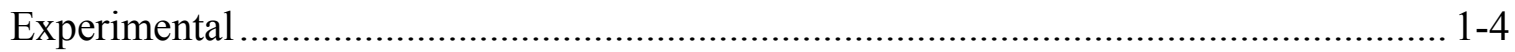

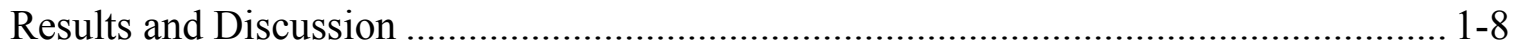

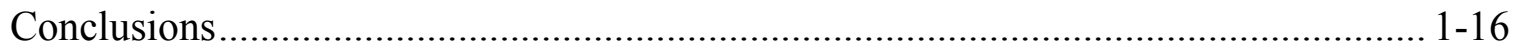

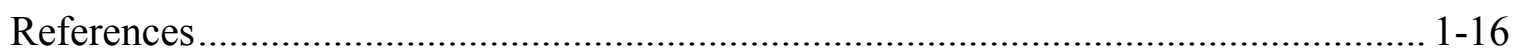

CHAPTER 2. CALCIUM LIGNOSULFONATE TRANSPORT THROUGH BEREA

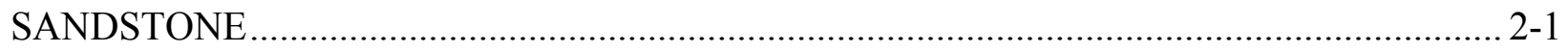

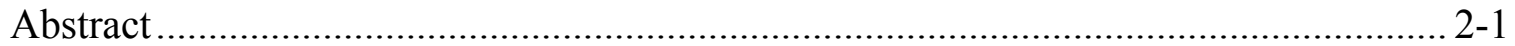

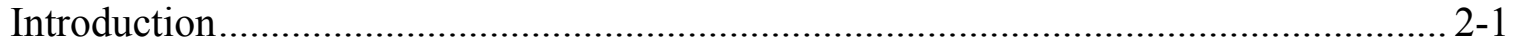

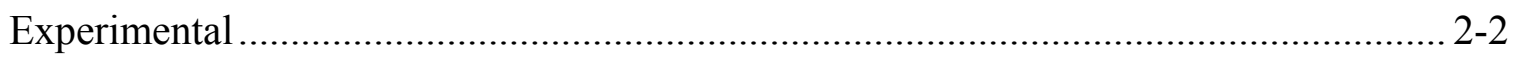

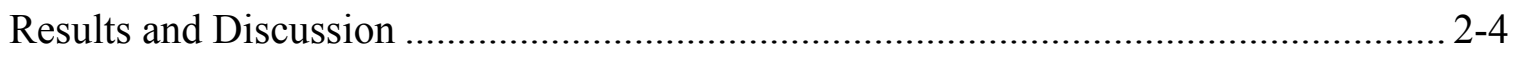

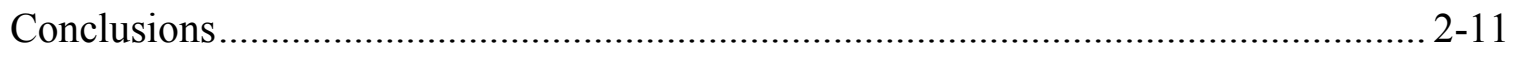

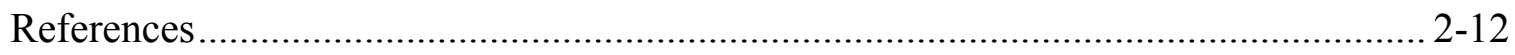

CHAPTER 3. SURFACTANT ADSORPTION AND DESORPTION ONTO FIVE MINERALS

AND THREE POROUS MEDIA …………………....................................................

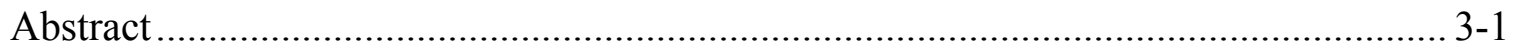

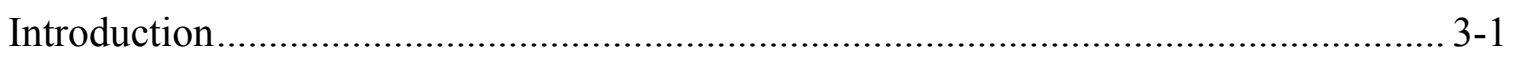

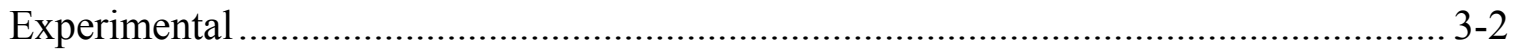

Results and Discussion .................................................................................. 3-5

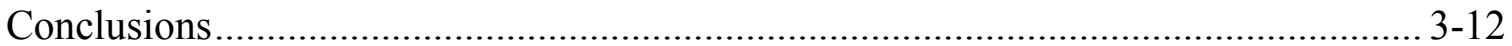

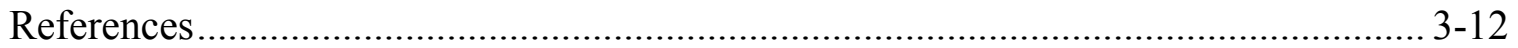


CHAPTER 4. COMPETITIVE ADSORPTION OF A HYBRID SURFACTANT SYSTEM ONTO FIVE MINERALS, BEREA SANDSTONE, AND LIMESTONE.

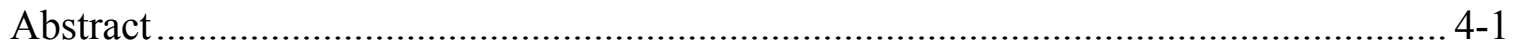

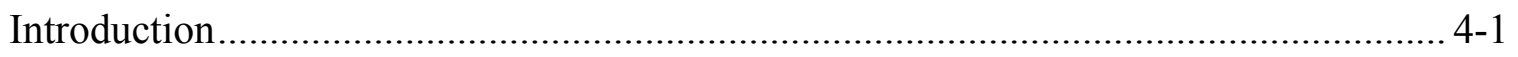

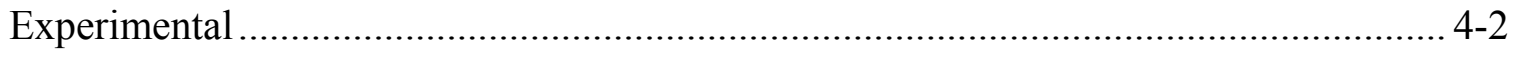

Results and Discussion ............................................................................... 4-11

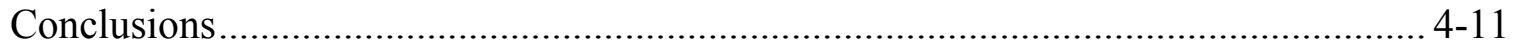

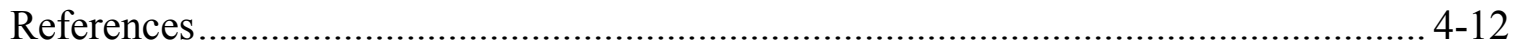

CHAPTER 5. INJECTIVITY LOSS: DEVELOPMENT OF GENERAL EQUATIONS TO QUANTIFY STRESS-SENSITIVITIES OF PERMEABILITY AND NON-DARCY

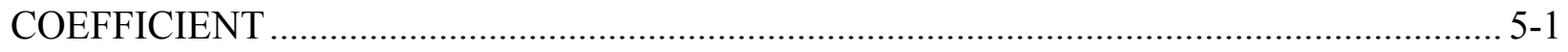

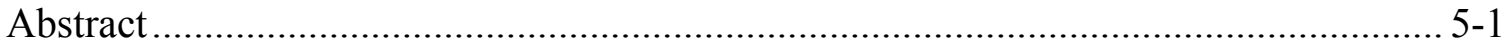

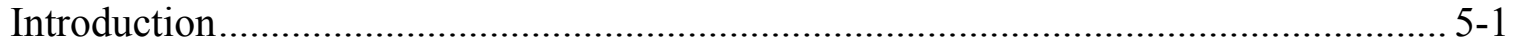

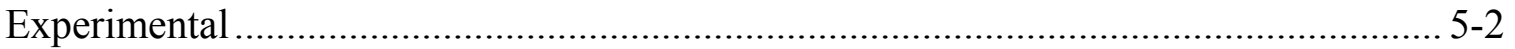

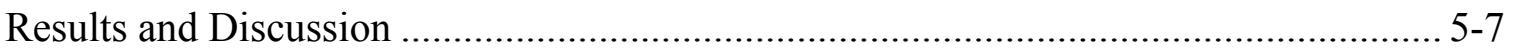

Conclusions ................................................................................................ 5-14

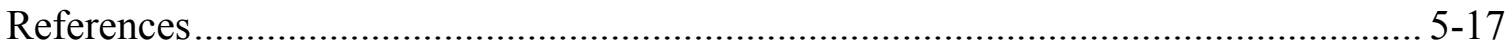

CHAPTER 6. INJECTIVITY LOSS: COMPARISON OF NON-DARCY FLOW OF $\mathrm{CO}_{2}$ AND

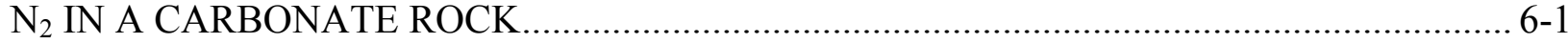

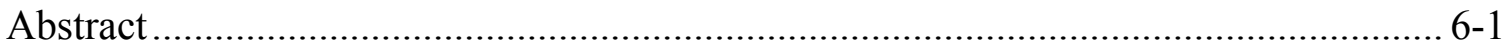

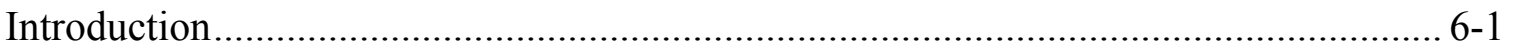

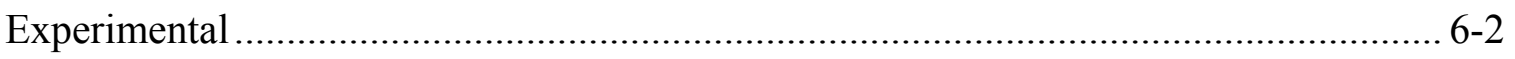

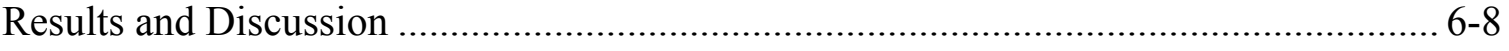

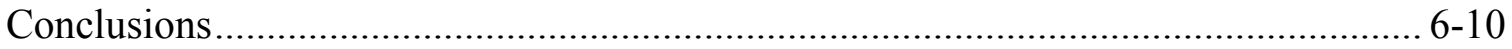

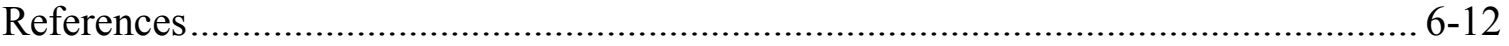




\section{List of Tables}

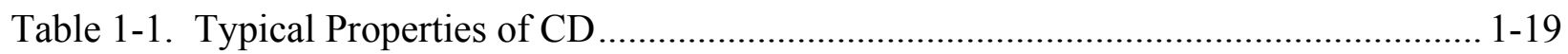

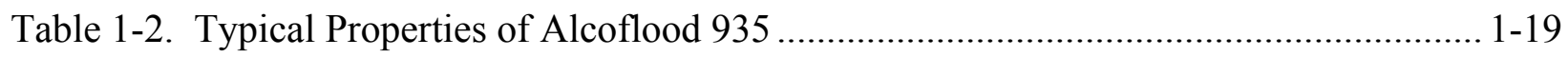

Table 1-3. Adsorptions in the Limestone.......................................................................... 1-19

Table1-4. IFT Values before and after Adsorption in the Limestone....................................... 1-19

Table 1-5. Stability before and after Adsorption in the Limestone ......................................... 1-20

Table 1-6. Adsorptions in the Berea Sandstone .................................................................. 1-20

Table 1-7. IFT before and after Adsorption in the Berea Sandstone ...................................... 1-20

Table 1-8. Stability before and after Adsorption in the Berea Sandstone ............................... 1-20

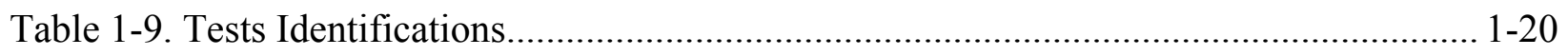

Table 1-10. The Results of Tests Exposed to the Light ....................................................... 1-22

Table 1-11. The Results of Tests in the Dark ………...................................................... 1-25

Table 2-1. Source and Main Composition of Five Minerals .................................................. 2-13

Table 2-2. Properties of Berea Core Samples ................................................................... 2-14

Table 2-3. Schedule of Tracer Breakthrough Experiments ..................................................... 2-14

Table 3-1. Summary of Mixture Components for Making Indicator Solution ......................... 3-14

Table 3-2. Parameters of Three Porous Media ………........................................................ 3-14

Table 3-3. Peak Absorbance of CD Solution..................................................................... 3-14

Table 3-4. 500mg/l CD Adsorption onto Three Porous Media …………................................ 3-14

Table 4-1. Standard Curve Equations of CD1045

with Different Concentration CLS................................................................................. 4-14

Table 4-2. Parameters of Berea Sandstone and Indiana Limestone........................................... 4-14

Table 4-3. Experiment Schedule of CLS, CD and Their Hybrid

Adsorption/Desorption onto Berea Sandstones and Limestone.................................................. 4-14 
Table 4-4. Equilibrium Concentration for Sandstone and Limestone with Starting Concentration of $500 \mathrm{mg} / 1$ of CD and $5000 \mathrm{mg} / 1$ of CLS

Table 4-5. IFT values before and after Adsorption for Sandstone and Limestone with Starting Concentration of $500 \mathrm{mg} / \mathrm{l}$ of CD and $5000 \mathrm{mg} / \mathrm{l}$ of CLS.

Table 4-6. Stability before and after Adsorption for Sandstone and Limestone with Starting Concentration of $500 \mathrm{mg} / \mathrm{l}$ of CD and $5000 \mathrm{mg} / \mathrm{l}$ of CLS

Table 5-1. Selected Experimental Conditions $5-18$

Table 5-2. Sample Specifications $5-19$

Table 5-3. Summary of Test Conditions for Each Sample $5-19$

Table 5-4. Example of Measured and Calculated Values $5-19$

Table 5-5. Summary of $k$ and $\beta$ of All the Tests... $5-20$

Table 6-1. Experiments and Calculated Values..... 6-13

Table 6-2. Relative Change of $\mathrm{k}$ and $\beta$ under Different Pore Pressures 6-13 


\section{List of Figures}

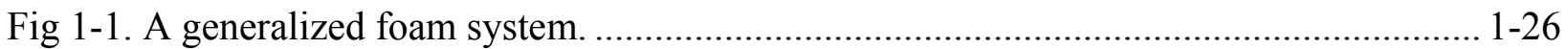

Fig 1-2. Image of $\mathrm{CO} 2$ foams in $\mathrm{CD}$ 0.025\% solution. ................................................... 1-26

Fig 1-3. Monomer (X1) and aggregate (micelle, $\mathrm{Xn}$ ) concentrations as a function of total concentration.

Fig. 1-4. Cartoon of capillary and drop used to determine IFT in the drop weight method...... 1-27

Fig. 1-5. Harkins-Brown correction factor for drop-weight method after Adamson. ............... 1-28

Fig. 1-6. Foam stability apparatus setup ....................................................................... 1-28

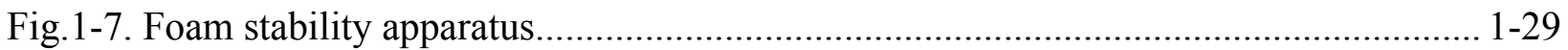

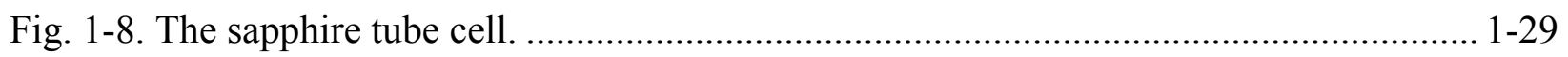

Fig 1-9. Example of a section of lignosulfonate molecule.................................................... 1-30

Fig. 1-10. CMC determination for CD.............................................................................. 1-30

Fig. 1-11 Salinity effect on IFT of CD solutions............................................................... 1-31

Fig. 1-12. IFT vs. temperature and surfactant concentration in CD solutions.......................... 1-31

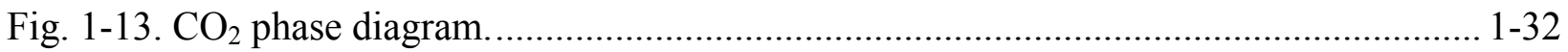

Fig. 1-14. IFT vs. pressure and surfactant concentration.................................................... 1-32

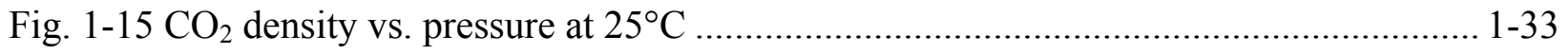

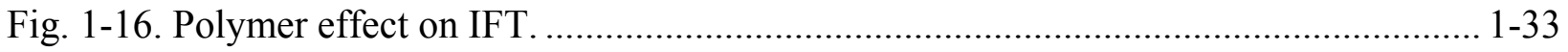

Fig. 1-17. Dynamic IFT for polymer in CD1045 brine solution. ........................................... 1-34

Fig. 1-18. Polymer effect on IFT in CD brine solution. .......................................................... 1-34

Fig. 1-19. pH effect on IFT for CD in 2\% brine solution. ..................................................... 1-35

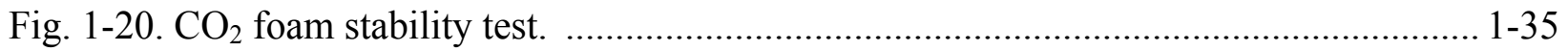

Fig. 1-21. Foam stability at different $\mathrm{CD}$ concentrations. ....................................................... 1-36

Fig. 1-22. Small volume changes occur with time from gravity drainage even for stable foams.1-36 
Fig. 1-23. Unstable foam bubbles are neither well defined nor homogeneous shapes. $1-37$

Fig. 1-24. Gravity drainage ..................................................................................... 1-37

Fig. 1-25. $\mathrm{CO}_{2}$ traversed the oil in a string of connected bubbles. .................................. 1-38

Fig. 1-26. Foam stability at different CD concentrations .......................................... 1-38

Fig.1-27. Salinity versus $\mathrm{CO}_{2}$ foam stability....................................................... 1-39

Fig. 1-28. Gravity drainage at different salinities................................................... 1-39

Fig. 1-29. Temperature effect on $\mathrm{CO}_{2}$ foam stability. .............................................. 1-40

Fig. 1-30. Pressure effect on $\mathrm{CO}_{2}$ foam stability....................................................... 1-41

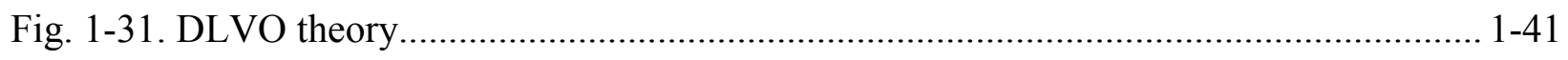

Fig. 1-32. Effect of HPAM concentration in 2\% brine solution after bubble generation......... 1-42

Fig. 1-33. Effect of HPAM concentration on $\mathrm{CO}_{2}$ coalescing in a solution of

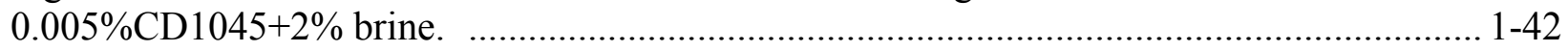

Fig. 1-34. Foam stability at $0.005 w t \%$ CD1045 in $2 \%$ brine. .......................................... 1-43

Fig. 1-35. Foam images at $0.005 \%$ CD1045 in 2\% brine................................................. 1-44 $1-43$

Fig. 1-36. Effect of indicated $\mathrm{pH}$ on $\mathrm{CO}_{2}$ foam stability at $0.05 \% \mathrm{CD}$ in $2 \%$ brine............... 1-44

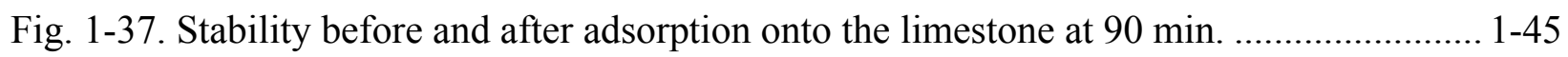

Fig. 1-38 Stability before and after adsorption in the limestone for hybrid surfactant systems.1-45

Fig. 1-39. Stability before and after adsorption in the Berea sandstone for hybrid surfactant

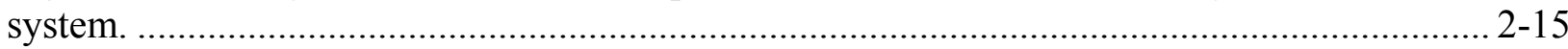

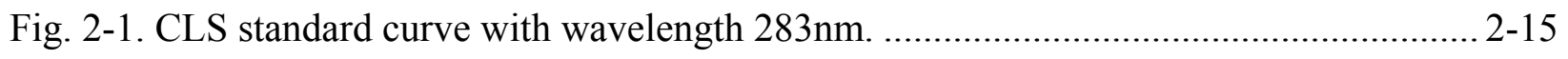

Fig. 2-2. Schematic diagram of flow-through method.................................................. 2-16

Fig. 2-3. Schematic diagram of circulation method.................................................... 2-16

Fig. 2-4. Schematic diagram of static adsorption. ..................................................... 2-17

Fig. 2-5Adsorption dynamic of CLS onto five minerals. ............................................... 2-17 
Fig. 2-6. Adsorption and desorption kinetics of CLS onto silica and kaolinite. $2-18$

Fig. 2-7. CLS adsorption isotherm onto four minerals. $2-18$

Fig. 2-8. CLS adsorption onto Berea sandstone. 2-19

Fig. 2-9. CLS adsorption onto Berea sandstone. 2-19

Fig. 2-10. Dispersion mechanisms of CLS through sandstone (Fetter, C.W.:Applied Hydrogeology., 2d ed., New York, Macmillan Publishing Company, 1988). $2-20$

Fig. 2-11. Characterization of Berea sandstone. $2-20$

Fig. 2-12. Effect of post-flush rate on CLS desorption from Berea sandstone. $2-20$

Fig. 2-13. Flow interruption experiment results. $2-21$

Fig. 2-14. Tracer flow through Berea sandstone. $2-21$

Fig. 2-15. Breakthrough curves during tracer injection.

Fig. 2-16. Interruption experiment results for experiment No.2

( stopped at 5.18 PV, 11.12PV).

Fig. 2-17. Comparison of breakthrough curves for first two experiment during brine injection. $2-23$

Fig. 2-18. Interruption experiment results for experiment No.3 (stopped at 5.78PV, 11.06PV).

Fig. 3-1. standard curve of CD at $295 \mathrm{~nm}$. $3-15$

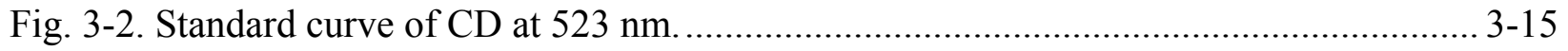

Fig. 3-3. CD adsorption process onto five adsorbents. .............................................. 3-16

Fig. 3-4. Adsorption density of CD onto five minerals (adsorption results). ....................... 3-16

Fig. 3-5. CD desorption from five minerals as a function of time................................... 3-17

Fig. 3-6. Adsorption density of CD onto five minerals (desorption results). ....................... 3-17

Fig. 3-7. Comparison of $\mathrm{CD}$ adsorption and desorption onto five minerals. ....................... 3-18

Fig. 3-8. Comparison of CD adsorption density onto five adsorbents.................................. 3-18 
Fig. 3-9. A normal plot of CD adsorption density with time (Berea).

Fig. 3-10. A semilog plot of CD adsorption density with time (Berea). ................................... 3-19

Fig. 3-11. Percent of CD desorbed from Berea sandstone vs. pore volume............................. 3-20

Fig. 3-12. CD desorption from Berea sandstone as a function of time. .................................. 3-20

Fig. 3-13. A normal plot of $\mathrm{CD}$ adsorption density with time (limestone). .............................. 3-21

Fig. 3-14. A semilog plot of CD 1045 adsorption onto limestone........................................... 3-21

Fig. 3-15. CD desorption from limestone vs. injection pore volume. ..................................... 3-22

Fig. 3-16. CD desorption from limestone as a function of time (direct flow) ......................... 3-22

Fig. 3-17. A normal plot of $\mathrm{CD}$ adsorption onto dolomite as a function of time. .................... 3-23

Fig. 3-18. A semiplot of CD adsorption onto dolomite as a function of time. .......................... 3-23

Fig. 3-19. CD concentration in solution as a function of time during its desorption from dolomite.3-24

Fig. 3-20. A semi-log plot of CD desorption from dolomite as a function of time. .................. 3-24

Fig. 3-21. Comparison of $\mathrm{CD}$ adsorption onto three porous media......................................... 3-25

Fig. 3-22. Surfactant loss due to adsorption onto rocks....................................................... 3-25

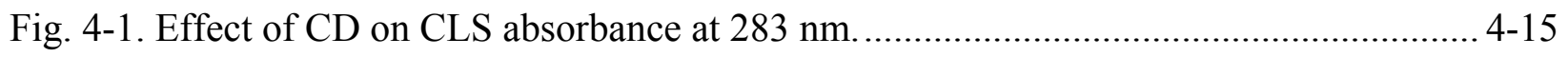

Fig. 4-2. CD standard curve at different concentration of CLS.............................................. 4-16

Fig.4-3. The relationship of CLS concentration and slope of fit equations............................. 4-16

Fig.4-4. The relationship of CLS concentration and intercept of fit equations. ....................... 4-17

Fig. 4-5. The sapphire tube cell with capillary inlet. ........................................................... 4-18

Fig. 4-6. CD adsorption density comparison for only CD and mixture $(500 \mathrm{mg} / \mathrm{l} \mathrm{CD}, 5,000 \mathrm{mg} / \mathrm{l}$ CLS, @ 40oC, 2\% brine)................................................................................................ 4-18

Fig. 4-7. CD adsorption reduction when mixing with CLS................................................. 4-19

Fig. 4-8. CLS adsorption density comparison for CLS and mixture solution. ........................ 4-19 
Fig. 4-9. CLS adsorption reduction when mixing with CD.

Fig. 4-10. CD concentration change versus time (sandstone). ............................................... 4-20

Fig. 4-11. CD adsorption onto sandstone versus time......................................................... 4-21

Fig. 4-12. CLS concentration change with time (Berea sandstone). ....................................... 4-21

Fig. 4-13. CLS adsorption change with time onto sandstone ............................................ 4-22

Fig. 4-14. Adsorption profile comparison of CD and CLS during mixture injection................ 4-22

Fig. 4-15. Desorption profile comparison of CD and CLS after mixed injection. ................... 4-23

Fig. 4-16. CD adsorption and CLS desorption from Berea sandstone...................................... 4-23

Fig. 4-17. CD and CLS desorption from Berea sandstone. ................................................... 4-24

Fig. 4-18. Comparison of CD and CLS adsorption onto Berea sandstone for different schemes.4-24

Fig. 4-19. Relative adsorption of CD1045 and CLS onto Berea sandstone for different schemes.4-25

Fig. 4-20. CD1045 concentration vs. time (limestone).......................................................... 4-25

Fig. 4-21. CD adsorption vs. time onto limestone. ………................................................. 4-26

Fig. 4-22. CLS concentration vs. time (limestone) …….................................................... 4-26

Fig. 4-23. CLS adsorption density vs. time onto limestone..................................................... 4-27

Fig. 4-24. Adsorption profile comparison of CD and CLS mixture on limestone.................... 4-27

Fig. 4-25. Comparison of $\mathrm{CD}$ adsorption onto limestone using

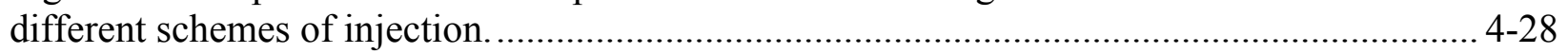

Fig.4-26. Relative adsorption of CD1045 and CLS onto limestone....................................... 4-28

Fig.4-27. Stability before and after adsorption in the limestone............................................ 4-29

Fig.4-28. Stability before and after adsorption in the limestone

for hybrid surfactant system..

Fig. 4-29. Stability before and after adsorption in the Berea sandstone

for hybrid surfactant system. 4-30

Fig. 5-1. Setup of the experiment. $5-25$ 
Fig. 5-2. Example of determining $\mathrm{k}$ and $\beta$ from a non-Darcy flow experiment.

Fig. 5-3. Effective stress vs. permeability relations under hydrostatic in-situ stress field at $100^{\circ} \mathrm{F}, 150^{\circ} \mathrm{F}$ and $200^{\circ} \mathrm{F}$ in four samples.

Fig. 5-4. Effective stress vs. non-Darcy flow coefficient relations under hydrostatic in-situ stress field at $100^{\circ} \mathrm{F}, 150^{\circ} \mathrm{F}$ and $200^{\circ} \mathrm{F}$ in four samples.

Fig. 5-5. $\mathrm{k}\left(\sigma_{\mathrm{eff}}\right)$ and $\beta\left(\sigma_{\text {eff }}\right)$ relations under differential in-situ stress fields at three temperatures in Dakota sandstone.

Fig. 5-6. $\sigma_{\text {eff }}-\mathrm{k}$ and $\sigma_{\text {eff }}-\beta$ relations under different reservoir pressures at $100^{\circ} \mathrm{F}$ in high-perm Indian limestone.

Fig. 5-7. Relations between stress sensitivities and their nominal non-Darcy flow parameters. 5-29

Fig. 5-8. $\tau$-k and $\tau-\beta$ curves under temperatures of $100^{\circ} \mathrm{F}, 150^{\circ} \mathrm{F}$ and $200^{\circ} \mathrm{F}$ in Dakota sandstone: widely scattered distribution of the points indicates poor correlation.

Fig. 5-9. $\tau-\mathrm{k}$ and $\tau-\beta$ curves under outlet BPR pressures of 500 psi and 1500 psi in high-perm Indiana limestone: widely scattered distribution of the points indicates poor correlation.

Fig. 6-1. Comparison of trend lines in $\mathrm{N}_{2}$ and $\mathrm{CO}_{2}$ flooding through low permeability Indiana limestone under effective stress of about 3300 psi.

Fig. 6-2 Relationship between shear stress and non-Darcy flow parameters in IL301 at temperature of $100^{\circ} \mathrm{F}$ : (a) permeability, (b) non-Darcy flow coefficient.

Fig. 6-3. Comparison of trend lines in $\mathrm{N}_{2}$ and $\mathrm{CO}_{2}$ flooding in IL301 under effective stress of about 5300 psi. 6-16

Fig. 6-4. Comparison of $\left(\mathrm{k}_{\mathrm{N} 2}, \beta_{\mathrm{N} 2}\right)$ and $\left(\mathrm{k}_{\mathrm{CO} 2}, \beta_{\mathrm{CO} 2}\right)$ under different effective stresses. 6-16

Fig. 6-5. Comparison of pore pressure effects on permeability in $\mathrm{N}_{2}$ and $\mathrm{CO}_{2}$ flooding.

Fig. 6-6. Comparison of pore pressure effects on non-Darcy flow coefficient in $\mathrm{N}_{2}$ and $\mathrm{CO}_{2}$ flooding. 6-18

Fig. 6-7. Comparison of temperature effects on permeability in $\mathrm{N}_{2}$ and $\mathrm{CO}_{2}$ flooding. 6-19

Fig. 6-8. Comparison of temperature effects on non-Darcy flow coefficient in $\mathrm{N}_{2}$ and $\mathrm{CO}_{2}$ flooding. 
Fig. 6-9. Stable thermal equilibrium in $\mathrm{N}_{2}$ flooding experiments in test $\mathrm{N}_{2} \_100 \_4000 \_4000 \_500$

in IL301: no change of temperature with the increase of flow rate and inlet pressure.

Fig. 6-10. Unstable thermal equilibrium in $\mathrm{CO}_{2}$ flooding experiments in test $\mathrm{CO}_{2}$ 100_4000_2000_500 in IL301: temperature decreases rapidly with increase of flow rate and inlet pressure.

Fig. 6-11. Unstable thermal equilibrium in $\mathrm{CO}_{2}$ flooding experiments in test $\mathrm{CO}_{2}$ 100_6000_3000_1000 in IL301: temperature started decreasing rapidly at relatively low flow rate (outlet pressure was set at about $1000 \mathrm{psi}$ ).

Fig. 6-12. Cooling effect started when bottomhole conditions approached the critical conditions of $\mathrm{CO}_{2}$. Bottomhole pressure did not recover. 


\section{Executive Summary}

The third annual report of "Improving $\mathrm{CO}_{2}$ Efficiency for Recovery Oil in Heterogeneous Reservoirs" presents results of laboratory studies with related analytical models for improved oil recovery. All studies have been undertaken with the express intention to optimize utilization and extend the practice of $\mathrm{CO}_{2}$ flooding to a wider range of reservoirs.

Last year we reported on the effects of salinity, pressure, temperature, surfactant concentration, and the presence of oil on the interfacial tension (IFT) and carbon dioxide $\left(\mathrm{CO}_{2}\right)$ foam stability. This year behavior at low concentrations of the surfactant Chaser International CD1045 ${ }^{\mathrm{TM}}$ (CD) versus different salinity, pressure and temperature are reported in Chapter 1. Also the effects of $\mathrm{pH}$ and polymer (hydrolyzed polyacrylamide- $\mathrm{HPAM}$ ) and $\mathrm{CO}_{2}$ foam stability after adsorption in the core are reported. The results of this work show IFT to be sensitive to the changes of temperature, pressure, surfactant concentration and presence of polymer in brine but relatively insensitive to salinity, $\mathrm{pH}$ and polymer in a surfactant solution. $\mathrm{CO}_{2}$ foam stability has been determined to be sensitive to salinity, $\mathrm{pH}$, polymer, pressure, temperature, oil presence and surfactant concentration at low surfactant concentrations, but insensitive to each of the above variables at higher surfactant concentrations. Coinjection of CLS and CD is favorable to generate $\mathrm{CO}_{2}$ foam in the core at low concentration of $\mathrm{CD}$ brine solutions. But chromatographic effects in the core were observed for $\mathrm{CD}$ brine solutions.

Calcium lignosulfonate (CLS) and CD, shown respectively to be a good sacrificial/enhancing agent and a good foaming agent in earlier reports projects, were selected to be studied for equilibrium and kinetics of surfactant adsorption onto reservoir core. The last annual report described adsorption equilibrium of CLS onto Berea sandstone and five minerals (silica, montmorillonite, kaolinite, dolomite, and calcite) common in reservoir rocks. The effects of surfactant concentration, salinity, temperature, $\mathrm{pH}$, and injection rate on equilibrium adsorption density were determined. This report includes CLS transport mechanisms through sandstone, description of the adsorption of $\mathrm{CD}$ and $\mathrm{CD} / \mathrm{CLS}$ onto three porous media (sandstone, limestone and dolomite) and five minerals, and the effect of adsorption on foam stability. 
The adsorption kinetics of CLS in porous Berea sandstone and non-porous minerals are compared in Chapter 2 by monitoring adsorption density change with time. Results show that adsorption requires a much longer time for the porous versus non-porous medium. CLS adsorption onto sandstone can be divided into three regions: adsorption controlled by dispersion, adsorption controlled by diffusion and adsorption equilibrium. $\mathrm{NaI}$ tracer used to characterize the sandstone had similar trends to earlier results for the CLS desorption process, suggesting a dual porosity model to simulate flow through Berea sandstone.

The kinetics and equilibrium test for CD adsorption onto five non-porous minerals and three porous media are reported in Chapter 3. CD adsorption and desorption onto non-porous minerals can be established in less than one hour with adsorption densities ranging from 0.4 to $1.2 \mathrm{mg}$ of CD per $\mathrm{g}$ of mineral in decreasing order of montmorillonite, dolomite, kaolinite, silica and calcite. The surfactant adsorption onto three porous media takes much longer than one hour, with Berea sandstone requiring the longest time.

In Chapter 4 comparisons of static adsorption of CLS, CD, and CLS/CD mixtures onto five pure minerals show that the presence of CLS decreased the adsorption of CD onto the five minerals decreases by 20 to $70 \%$. Dynamic CLS/CD mixture adsorption tests onto Berea sandstone and Indian limestone cores showed that competitive adsorption between CD and CLS generally takes several days to reach equilibrium. Foam stability and interfacial tension tests on both injected and effluent samples were performed which showed that both foam stability and IFT decreased due to adsorption. Also it appears that there is a chromatographic effect on the surfactants in flow through porous media.

In the last annual report, typical field conditions of $\mathrm{CO}_{2}$ flooding were summarized, similar laboratory experimental parameters were determined, experimental facilities were updated and high flow rate gas flooding experiments were completed on five representative rocks versus pressure, temperature, and flow rate. Related theoretical formulas were developed, and experimental data processed, resulting in 60 correlations based on the measured permeabilities and non-Darcy coefficients. This report documents progress in developing general equations for the stress sensitivity on non-Darcy parameters (permeability and non-Darcy coefficient), and the 
confirmation of multiphase flow induced by high-flow rate as a mechanism for injectivity loss in $\mathrm{CO}_{2}$ flooding.

A general equation is defined in Chapter 5 based on the aforementioned 60 general equations of permeability stress sensitivity and non-Darcy coefficient stress sensitivity and definitions of nominal permeability, nominal non-Darcy coefficient, permeability stress sensitivity, and nonDarcy coefficient stress sensitivity. The equations of stress sensitivity are independent of pressure, temperature, and rock properties and existing empirical correlations of the nominal permeability and nominal non-Darcy coefficient can be used when laboratory data are not available. This provides a tool to quantify the change of permeability and non-Darcy coefficient due to change of effective stress resulted from reservoir injection and/or production.

Results presented in Chapter 6 compare the high rate flow behavior of $\mathrm{N}_{2}$ and $\mathrm{CO}_{2}$ and show that the permeability and non-Darcy coefficient are different under similar experimental conditions. Under normal reservoir conditions $\mathrm{N}_{2}$ does not deviate significantly from an ideal gas while $\mathrm{CO}_{2}$ might be gas like, liquid like, described as supercritical fluid, or transforming from one state to another. This phenomenon creates unstable regions and is difficult to predict results, including additional phases, rapid pressure changes, and localized reservoir temperature reduction. Each condition can result in increased pressure gradients, reduced injectivity and productivity, and formation damage. 


\title{
CHAPTER 1. INTERFACIAL TENSION AND $\mathrm{CO}_{2}$ FOAM STABILITY
}

\begin{abstract}
The foam stability apparatus, a bubble tube, is used to screen surfactant candidates for $\mathrm{CO}_{2}$ application and optimizing surfactant concentrations. The interfacial tension (IFT) determined between high-pressure, high-temperature (HPHT) $\mathrm{CO}_{2}$ and brine/surfactant solution using a drop weight method, critical micelle concentration (CMC) determined by plotting IFT versus concentration, and $\mathrm{CO}_{2}$ foam stability at reservoir temperature and pressure conditions determined visually are all performed in this bubble tube apparatus. This system has been used to determine effects of salinity, pressure, temperature, surfactant concentration, $\mathrm{pH}$, polymer and the presence of oil on IFT and $\mathrm{CO}_{2}$ foam stability. All tests in this section were performed with the surfactant CD1045 ${ }^{\mathrm{TM}}$ (CD). IFT has been determined to:
\end{abstract}

1. Be insensitive to brine concentration over a wide range with a minimum around $10 \%$.

2. Decrease with surfactant concentration below the CMC and be essentially constant above the CMC.

3. Increase with the increase of temperature.

4. Decrease with the increase of pressure.

5. Decrease after adding polymer into brine solution but be insensitive to polymer after adding polymer to $\mathrm{CD}$ brine solution.

6. Be insensitive to $\mathrm{pH}$ change from 1 to 12 .

Stability of $\mathrm{CO}_{2}$ foam has been determined to:

1. Be insensitive to brine concentration over a wide range of concentrations.

2. Increase with surfactant concentration to the CMC.

3. Decrease with increase of temperature.

4. Decrease with pressure increase at the low surfactant concentration of $0.005 \mathrm{wt} \%$, and be insensitive to pressure at $\mathrm{CD}$ concentrations of $0.025 \mathrm{wt} \%$ and above.

5. Increase with polymer by impeding lamellae thinning due to its high viscosity.

6. Decrease with $\mathrm{pH}$ decrease at the low surfactant concentration of $0.005 \mathrm{wt} \%$ and be insensitive to $\mathrm{pH}$ at $\mathrm{CD}$ concentration of $0.05 \mathrm{wt} \%$ and above. 
In general, bubbles in stable foams are polyhedral, smaller and more homogeneous than in unstable foams. Foam volume in stable foams decreases with time from gravity drainage due to lamella thinning. In the presence of oil, $\mathrm{CO}_{2}$ bubbles have irregular shapes and it appears that gravity drainage is impeded, even while the irregular bubbles coalesce.

\section{Introduction}

A foam is a special kind of colloidal dispersion: one in which a gas is dispersed in a continuous liquid phase. ${ }^{1}$ For high pressure $\mathrm{CO}_{2}$ foam, the dispersed phase is dense $\mathrm{CO}_{2}$ whose density ranges from 0.5 to $0.9 \mathrm{~g} / \mathrm{cm}^{3}$, which is strictly emulsion, to which Wellington gave a special name: "foamulsion." The term foam is retained here because it is the conventional way to identify $\mathrm{CO}_{2}$ foam in the petroleum industry. In our experiment, we generated $\mathrm{CO}_{2}$ foam whose dispersed phase is dense $\mathrm{CO}_{2}$. The dispersed phase is sometimes referred to as the internal phase, and the continuous phase as the external phase. A two-dimensional slice of a general foam system is shown in Fig 1-1. ${ }^{1}$ The general foam structure is contained on the bottom by the bulk liquid and on the upper side by a second bulk phase (in the $\mathrm{CO}_{2}$ foam, dense $\mathrm{CO}_{2}$ ). Within the magnified region, the various parts of the foam structure are clarified. The upper phase $\left(\mathrm{CO}_{2}\right.$ foam-dense $\mathrm{CO}_{2}$ ) is separated from the thin liquid-film, by a two-dimensional interface. In a persistent foam, the spherical bubbles become transformed into foam cells, polyhedral (almost dodecahedral) separated by almost flat liquid films, which result from the surface tensions. A similar $\mathrm{CO}_{2}$ foam structure (Fig 1-2) was observed in our foam static stability tests. Dictated by mathematical convenience, the physical behavior of this interfacial region is approximated by a two-dimensional surface phase (the Gibbs surface).

Laurier L. Schramm ${ }^{1}$ defined a lamella as the region that encompasses the thin film, the two interfaces on either side of the thin film, and part of the junction to other lamellae. The connection of three lamellae, at a $120^{\circ}$ angle, is referred to as the Plateau border. Figure 1-1 represents only a two-dimensional slice, as the Plateau border extends perpendicularly, out of the page. In three dimensions, four Plateau borders meet at a point at the tetrahedral angle, approximately $109^{\circ}$. 
The surfactants are added to the solution to generate a more stable foam by reducing interfacial tension and to form stable lamellae. The reason for this is that the adsorption of surfactant at the gas-liquid interface promotes thin-film stability between the bubbles and lends a certain persistence to the foam structure. Thus, when two bubbles of gas approach, the liquid-film thins down to a persistent lamella instead of rupturing at the point of closest approach.

Foams have been of great practical interest because of their widespread occurrence and their important properties. In the oil and gas sector, foams may be applied or encountered at all stages in the petroleum recovery and processing industry such as oil well drilling, injection, oil well production, and process plant foams. ${ }^{1}$ The idea of using foam for mobility control was proposed and patented by Bond and Holbrook in 1958. ${ }^{2}$ Fried conducted foam drive experiments and reported a sharp pressure drop across the foam bank and reduced gas mobility through porous media. ${ }^{3}$ There have been a number of reviews on foam research that include Heller and Taber, ${ }^{4}$ Heller et al., ${ }^{5}$ Marsden, ${ }^{6}$ and Hirasaki. ${ }^{7,8}$ The apparent viscosity of $\mathrm{CO}_{2}$ foam is much higher than that of dense $\mathrm{CO}_{2}$. The $\mathrm{CO}_{2}$ foam will increase the displacing fluid's apparent viscosity and improve the oil recovery by decreasing mobility. Using surfactants will generate more stable $\mathrm{CO}_{2}$ foam that will reduce viscous fingering, improve sweep efficiency, and if successful, improve oil recovery compared to $\mathrm{CO}_{2}$ and water.

Screening surfactant candidates and obtaining the optimum formation for $\mathrm{CO}_{2}$ floods are important. The properties of foam generated by different surfactants were determined using a high-pressure test apparatus constructed in our laboratory. ${ }^{9}$ The optimum surfactant mixture and its concentration were determined by comparing the foaming ability and the foam stability of different surfactants.

The study of surfactant interfacial tension and $\mathrm{CO}_{2}$ foam stability at in-situ conditions will provide general information about the properties of $\mathrm{CO}_{2}$ foam and the baseline properties of $\mathrm{CD}$ over a wide range of pressure, temperature, salinity, $\mathrm{pH}$ and the presence of oil. This data can be used to develop a CD-co-surfactant system that has the appropriate physical properties and favorable economic potential for field application. 


\section{Experimental}

\section{Definitions. $^{10}$}

Interfacial tension (IFT): Surface tension is a measurement of the cohesive energy present at an interface. The molecules of a liquid attract each other. The interactions of a molecule in the bulk of a liquid are balanced by an equal attractive force in all directions. Molecules on the surface of a liquid experience an imbalance of forces as indicated below.

The net effect of this situation is the presence of free energy at the surface. The excess energy is called surface free energy and can be quantified as a measurement of energy/area. It is also possible to describe this situation as having a line tension or surface tension quantified as a force/length measurement. The common units for surface tension are milliNewtons/meter $(\mathrm{mN} / \mathrm{m})$ or dynes/centimeter. It is useful in analyzing foaming, spreading, emulsification, wettability and other fluid characteristics. Conventionally, if one of the fluids is the vapor phase of a liquid being tested the measurement is referred to as surface tension ( $\sigma$ or "gamma"). If the surface investigated is the interface of two liquids, the measurement is referred to as the interfacial tension. In either case the more dense fluid is referred to herein as the "heavy phase" and the less dense fluid is referred to as the "light phase."

Critical micelle concentration (CMC). ${ }^{10}$

When the monomer concentration, X1, approaches exp $\left[-\left(\mu_{1}{ }^{o}-\mu_{n}{ }^{o}\right) / k T\right]$, it can increase no further (Fig 1-3). The monomer concentration (X1) crit at which this occurs is referred to as the critical aggregation concentration (CAC) though it is common to use the more conventional term critical micelle concentration (CMC) to demote the critical concentration of all self-assembled structures.

\section{IFT Measurement Methods. Drop Weight Method: ${ }^{11-17}$}

The drop weight method of measuring the interfacial tension of liquid with respect to air (dense $\mathrm{CO}_{2}$ in $\mathrm{CO}_{2}$ foam) consists of determining the number of drops falling from a capillary (Fig 1-4). The drops are allowed to fall into a container until enough have been collected so that the weight per drop can be determined accurately. The principle of the method is that the size of the drop 
falling from a capillary tube depends on the surface tension of the liquid. The maximum of liquid weight $\mathrm{W}$, which can hang from a capillary tube with radius $r$ without falling, depends on the surface tension as observations of falling drops show that a considerable portion of the drop (up to $40 \%$ ) may remain attached to the capillary end. This effect is compensated with HarkingBrown correction factor, $f$, as described by Adamson ${ }^{2}$ (Fig 1-5),

$$
\mathrm{W}=\Delta \mathrm{mg}
$$

where

$$
\begin{array}{clll}
\Delta \mathrm{m} & = & \text { differential mass between the two fluids, } \mathrm{g} \\
\mathrm{g} & = & \text { gravitational force, } \mathrm{cm} / \mathrm{sec}^{2} \\
\mathrm{r} & = & \text { needle radius, } \mathrm{cm} \\
\sigma & = & \text { IFT }\left(\mathrm{CO}_{2} \text { and aqueous solution in this study }\right) \text {, dynes } / \mathrm{cm}
\end{array}
$$

$$
\text { or }
$$$$
\frac{4}{3} \pi R^{3}\left(\rho_{\text {surf }}-\rho_{\mathrm{CO}_{2}}\right) g=2 \pi r \sigma f
$$

$$
\text { where }
$$

$\begin{array}{lll}\mathrm{R} & = & \text { average bubble radius, } \mathrm{cm} \\ \rho & = & \text { fluid densities, } \mathrm{g} / \mathrm{cm}^{3} \\ \mathrm{f} & = & \text { correction factor }\end{array}$

This factor takes into account effects of attraction to the end of the tube and imperfections in the system. It ranges from 0.5 to 1.0 (see Fig. 1-5). Most of the experiments in this study exhibit $f$ in the range from 0.5 to 0.7 . This is a fairly accurate method and perhaps the most convenient in the laboratory for measuring both gas-liquid and liquid-liquid interfacial tensions. Design and construction of the apparatus was based on this method.

\section{Apparatus and Experimental Procedures.}

The foam stability apparatus built in the laboratory was used for testing surfactant properties at high pressure, thus allowing the evaluation of these solutions for reservoir use. An earlier stability apparatus was modified with the following additions:

1. A protective frame was constructed,

2. The valves were fixed on a panel to reduce vibration (especial sapphire tube), and 
3. The system was simplified without changing its functions (Figs. 1-6 through 1-8).

The apparatus consists of a $\mathrm{CO}_{2}$ source tank, a visual cell made from a transparent sapphire tube, an oil/surfactant-solution cylinder, a positive displacement pump and a cathetometer for measuring the level of bubble decay versus elapsed time (Fig. 1-6). The $\mathrm{CO}_{2}$ tank and the sapphire tube high-pressure cell are major parts of the system that are contained in a temperature controlled water bath (Figs. 1-7 \& 1-8). The pump and the oil/surfactant-solution cylinder are installed outside the water bath and their temperatures are maintained at the test temperature through an independent temperature control system.

During the stability experiment, the sapphire visual cell (Fig 1-8) was first filled with the solution to be tested. The aqueous system was brought to the desired pressure by means of the Ruska positive displacement pump. The pressure difference between the $\mathrm{CO}_{2}$ tank and the oil/surfactant-solution tank was determined by a Honeywell pressure transducer and brought to zero by fine adjustment of a Ruska positive displacement pump. At this point a valve at the bottom of the water tank was opened that allows flow of $\mathrm{CO}_{2}$ into the surfactant as the pump is driven backward, causing the withdrawal of surfactant solution from the sapphire cell and into the oil/surfactant-solution tank. This drew the dense $\mathrm{CO}_{2}$ upward through a needle at the lower end of the cell. Depending on the effectiveness of surfactants, the bubbles either formed a layer of foam at the top of the sapphire tube or coalesced into a clear layer of dense $\mathrm{CO}_{2}$. After a standard volume of $\mathrm{CO}_{2}$ (1.75 $\mathrm{cc}$ in one hour) was introduced into the sapphire tube, the pump was stopped and the stability of formed foam was measured. When the experiment was finished, the surfactant portion of the contents of the sapphire tube was discarded. The $\mathrm{CO}_{2}$ portion was bled out into the atmosphere. Finally, the sapphire tube was thoroughly rinsed with distilled water.

Foam stability is defined as the fraction of the bubbles that stay intact as a foam layer at the top of the cell. The stability of the foam is obtained in terms of foam decay by measuring the change in the percentage of total injected $\mathrm{CO}_{2}$ as foam versus time since the end of $\mathrm{CO}_{2}$ being bubbled through the surfactant solution. This test also provided the measurement techniques for calculating the interfacial tension between surfactants and dense $\mathrm{CO}_{2}$. This was determined by 
counting the number of bubbles produced at the needle per volume of $\mathrm{CO}_{2}$ injected.

\section{Chemicals.}

Surfactants: CD and calcium lignosulfonate (CLS) are the selected surfactants for this study. CD was identified as one of the best foaming agents in several other studies. ${ }^{18-20}$ It was supplied by Chaser International as $46.7 \mathrm{wt} \%$ active aqueous solution. Its typical properties are given in Table 1-1.

CLS (Lignosite 100 ${ }^{\mathrm{TM}}$ ) was obtained from the Georgia-Pacific Corporation. This product is produced by sulfonation of softwood lignin and is provided in a brown powder form by the company. The structure of a section of lignosulfonate is given in Fig 1-9. Sodium lignosulfonate (SLS) in solution with $46 \%$ active, was obtained from the polychemicals department, Westvaco.

Polymer: Alcoflood 935 is an anionic partially hydrolyzed polyacrylamide (HPAM) copolymer, supplied by Ciba Specialty Chemicals Corporation. Its typical properties are given in Table 1-1.

Other Chemicals: Sodium chloride $(\mathrm{NaCl})$ : A.C.S. reagent, $99+\%$, from Aldrich Chemical Company, Inc.

Calcium chloride dehydrate $\left(\mathrm{CaCl}_{2} \cdot \mathrm{H}_{2} \mathrm{O}\right)$ : A.C.S. reagent, $98+\%$, from Aldrich Chemical Company, Inc.

Nitric acid $\left(\mathrm{HNO}_{3}\right)$ : AR select ${ }^{\mathrm{TM}}, 70 \%$, Mallinckrodt

Sodium hydroxide $(\mathrm{NaOH})$ : A.C.S. reagent, $98.6 \%$, Mallinckrodt

All aqueous solutions had the weight ratio of $3: 1 \mathrm{NaCl}: \mathrm{CaCl}_{2} \cdot \mathrm{H}_{2} \mathrm{O}$ and unless stated otherwise were $2 \mathrm{wt} \%$ brines.

Oil: The light mineral oil (paraffin oil, light) was obtained from Fisher Chemical, Fisher Scientific and had a density of $0.8429 \mathrm{~g} / \mathrm{cm}^{3}$ and average molecular weight of $380 \mathrm{~g} / \mathrm{mole}$ (measured by GC). 


\section{Results and Discussion}

\section{Interfacial Tension}

\section{Surfactant Concentration Effect}

The surfactants were dissolved in synthetic brine and IFT values are determined in the CO2 foam stability apparatus (Fig 1-6 through 1-8) and calculated using Eq. 1.3. For CD, IFT has been determined to decrease with surfactant concentration below CMC and to be essentially constant above the $\mathrm{CMC}$ (Fig. $3-1)$ at $25^{\circ} \mathrm{C}\left(77^{\circ} \mathrm{F}\right)$ and 1500 psig. The characteristic discontinuity in the plots of IFT against surfactant concentration can be observed. Conventionally, the corresponding surfactant concentration at this discontinuity corresponds to the CMC (see Fig. 1-10). The CMC of $\mathrm{CD}$ is around $0.06 \mathrm{wt} \%$.

Mechanisms for this trend for $\mathrm{CD}$ are as follows. At surfactant concentration below the CMC, the surfactant molecules are loosely integrated into the water structure. In the region of the CMC, the surfactant-water structure is changed in such a way that the surfactant molecules begin to build up their own structures - micelles in the interior and monolayer at the interface. Micelles are surfactant aggregates formed in which the hydrophobic sections of the surfactant are stuck together due to the limited solubility of surfactants in aqueous phase and van der Waals attraction among hydrophobic tails (or chains). The number of monomers aggregated at the interface remains the same but the number of micelles will increase when surfactant concentration above the $\mathrm{CMC}$ is increased. IFT is related to the number of monomers aggregated at the interface and is independent to the number of micelles. CMC determined by testing IFT is the measure of saturation of the monomer's adsorption at the interface, which may possibly be lower than the theoretical $\mathrm{CMC}$ value. ${ }^{10}$

\section{Salinity Effect}

A synthetic brine consisting of $\mathrm{NaCl}$ and $\mathrm{CaCl}_{2} \cdot \mathrm{H}_{2} \mathrm{O}$ with weight ratio $3: 1$ was used to dissolve surfactant $\mathrm{CD}$. In order to determine the optimum range of salinity values, IFT between the dense $\mathrm{CO}_{2}$ and the $\mathrm{CD}$ solution were measured at different salinities and $\mathrm{CD}$ concentrations at $1500 \mathrm{psig}$ and $77^{\circ} \mathrm{F}\left(25^{\circ} \mathrm{C}\right)$. 
According to colloid theory, the addition of salt will increase screening between head groups, lower the electrostatic repulsion and allow micellization at lower surfactant concentration. The higher salt concentration also lowers solubility of a hydrophobic chain's solubility by increasing the IFT. ${ }^{10}$

Figure 1-11 shows the results of the IFT measurements. For brine solutions, the IFT increased with salinity increased. But the IFT for the given surfactant concentration has been determined to be insensitive to brine concentration over a wide range with a minimum between 5 and $10 \mathrm{wt} \%$ brine, depending on surfactant concentration. The trends are similar for surfactant concentrations below and above the $\mathrm{CMC}$, which means that $\mathrm{CMC}$ is insensitive to the salinity. IFT will increase with salinity after brine concentration is higher than $10 \mathrm{wt} \%$, as the colloid theory predicts.

\section{Temperature Effect}

In the application of foams in the petroleum industry, the temperature is a very important parameter. Previous studies on the effect of temperature on IFT indicate that observed trends will

depend on the systems studied. ${ }^{14,22-25}$ This phenomenon has not previously been well explained. But according to colloidal theory, the fluidity of the hydrocarbon chains increases as temperature increases, which allow the chains to assume more favorable packing configurations to form micelles, lowering the CMC. As temperature continues to increase, however, the thermal motion of the chains increases to the point where the close packing arrangement is disrupted, causing the $\mathrm{CMC}$ to begin to increase. ${ }^{10}$

Experiments were conducted on aqueous $\mathrm{CD}$ and a dense $\mathrm{CO}_{2}$ system at different $\mathrm{CD}$ concentrations. The temperature at which experiments were conducted ranged from $25^{\circ} \mathrm{C}\left(77^{\circ} \mathrm{F}\right)$ to $75^{\circ} \mathrm{C}\left(167^{\circ} \mathrm{F}\right)$ at $1500 \mathrm{psig}$. The densities of the surfactant solution used in the calculating IFT were measured at atmospheric pressure. The results show that IFT decreased slightly and then increased with temperature with $\mathrm{CD}$ concentration at $0.05 \mathrm{wt} \%$ (close to $\mathrm{CMC}$ ) and above (Fig. 1-12). IFT mainly increased with temperature with CD concentration below $0.05 \mathrm{wt} \%$. Figure 112 clearly shows that the $\mathrm{CMC}$ of $\mathrm{CD}$ is between $0.05 \mathrm{wt} \%$ and $0.1 \mathrm{wt} \%$ and is insensitive to the temperature, which is different from the theoretical prediction. This is either because CMC for 
CD determined by IFT is possibly less than the theoretical CMC value or because there are more complex interactions among other compounds in the CD.

\section{Pressure Effect}

Previous studies about the effects of pressure on IFT observed that trends will depend on the system studied. ${ }^{14,22-30}$ Experiments were conducted on aqueous $\mathrm{CD}$ and a dense $\mathrm{CO}_{2}$ system at different $\mathrm{CD}$ concentrations. The pressure at which experiments were conducted ranged from 800 psig to 2000 psig at $25^{\circ} \mathrm{C}\left(77^{\circ} \mathrm{F}\right) \cdot \mathrm{CO}_{2}$ is a liquid (dense $\left.\mathrm{CO}_{2}\right)$ at 1100 psig and above, while it is gas at $800 \mathrm{psig}$ (Fig 1-13). The results showed that the IFT was dramatically lower at 800 psig compared to the higher pressures, 1100 psig and above (Fig. 1-14). This trend is thought to be mainly due to the increase of $\mathrm{CO}_{2}$ density with pressures (Fig 1-15).

\section{Polymer Effect}

It is generally expected that addition of polymer will lower the IFT of a system. ${ }^{10}$ Our results showed that adding polymer to brine solution will lower the IFT (Fig 1-16). But the extent of decreasing IFT at low concentration of polymer solution is greater than that at high concentration of polymer solution (Fig 1-16). It is difficult to obtain stable IFT values when HPAM is added to the brine solution. This might be due to an unstable orientation of HPAM at the interface of $\mathrm{CO}_{2}$ and HPAM solution. When HPAM to CD was added to brine solutions, the IFT values at the lower concentration (Fig 1-17) of HPAM decreased IFT and CMC while IFT and CMC did not change when a higher concentration of polymer was added (Fig 1-18). A possible explanation for this is that high molecular weight; polymer will adsorb at interface and accelerate the micellization, which results in lowering CMC and IFT when a small amount of polymer is in the solution. When a large amount of polymer exists in the solution, the steric repulsion between molecules of HPAM will be dominant, which will cause the solution to demicellizate and increase IFT.

\section{pH Effect}

The $\mathrm{pH}$ of $\mathrm{CD}$ brine solution was adjusted with $\mathrm{HNO}_{3}$ for $\mathrm{pH}<7$ and $\mathrm{NaOH}$ for $\mathrm{pH}>7$. Three $\mathrm{CD}$ concentrations are tested in this study. They are $0.005 \mathrm{wt} \%, 0.05 \mathrm{wt} \%$ and $0.1 \mathrm{wt} \%$ respectively.

$\mathrm{pH}$ effect on IFT highly depends on the type of surfactants in the aqueous phase. ${ }^{10}$ Our results 
showed that IFT changed less than $1 \mathrm{mN} / \mathrm{m}$ when changing the $\mathrm{pH}$ from 1 to 12 , which indicates that IFT is insensitive to $\mathrm{pH}$ change (Fig. 1-19). Ionic surfactants are sensitive to salinity and $\mathrm{pH}$ while nonionic surfactants are less sensitive to salinity and $\mathrm{pH} .{ }^{10}$ Thus, $\mathrm{CD}$ must contain some nonionic surfactants.

\section{$\mathrm{CO}_{2}$ Foam Stability}

Foam texture is an important parameter for understanding foam transport because foam microstructure or texture (i.e., the size of individual foam bubbles) has important effects on flow phenomena in porous media. $\mathrm{CO}_{2}$ static stability apparatus (Fig 1-6 through 1-8) can determine the size of bubbles while measuring foam stability and IFT. Thus it is important to conduct $\mathrm{CO}_{2}$ foam stability test. By nature, no foams are thermodynamically stable, as are other types of colloidal dispersions. Eventually they all collapse. But it is possible to make surfactantstabilized, static bubbles and films with lifetimes on the order of months and years if suitable surfactants are selected and applied in a carefully controlled environment. That is that foams are not thermodynamically stable but they can exhibit kinetic stability. Thus one concern is $\mathrm{CO}_{2}$ foam kinetic stability. $\mathrm{CO}_{2}$ foam stability is determined by measuring the change of the volume of $\mathrm{CO}_{2}$ foam for 90 minutes after bubbles generation is completed (Fig 1-20). Eq. 1.4 shows how to calculate the $\mathrm{CO}_{2}$ foam stability with the foam height $\mathrm{H} 2$.

$\mathrm{CO}_{2}$ foam stability $=\mathrm{H} 2$ at $90 \mathrm{~min} / \mathrm{H} 2$ at $0 \mathrm{~min}$ 1.4

\section{Effects of Surfactant Concentration on $\mathrm{CO}_{2}$ Foam Stability}

$\mathrm{CD}$ foam does not collapse at concentrations much lower than the CMC (see Fig 1-21). $\mathrm{CO}_{2}$ foam stability of $\mathrm{CD}$ is insensitive to surfactant concentration over a wide range, which indicates that $\mathrm{CD}$ is a good foaming agent for $\mathrm{CO}_{2}$-brine systems. Figure 1-22 is a good example showing the polyhedral shape of a stable $\mathrm{CO} 2$-foam. The bubble size is relatively small and homogeneous compared to those in unstable foams (compare Fig. 1-23).

An interesting phenomenon is that the height of the foam layer decreased a few per cent with time (Fig. 1-22) even though the number of bubbles was constant in the foam (Figs. 1-22 and 124). Generally the reduction in volume increases with the increase in surfactant concentration. This volume reduction occurs because liquid drains in the lamellae due to the force of gravity 
after foam generation. The liquid drains by flowing downward through the liquid films. As the lamellae fluid drains and goes to drier foam the shape of the bubbles changes from spherical to polyhedral. Draining continues until capillary forces are equal to gravity forces. At the plateau borders (lamellae intersections, see Fig. 1-1) the gas-liquid interface curvature increases. The increased curvature generates a low-pressure region in the plateau border area. Because the interface is flat along the thin-film region, a higher pressure resides here. This pressure difference forces liquid to flow toward the plateau borders (Fig 1-1) and causes thinning of the films and motion in the foam.

\section{Oil Presence}

In the presence of oil, the mechanisms of foam stability are more complex than without oil. The density of light mineral oil is higher than the dense $\mathrm{CO}_{2}$ and less than $\mathrm{CD}$ solution at $25^{\circ} \mathrm{C}\left(77^{\circ} \mathrm{F}\right)$ and 1500 psig. Three mililiters of light mineral oil were injected into the sapphire tube. The generated $\mathrm{CO}_{2}$ had to pass through a layer of brine and then a layer of oil. The $\mathrm{CO}_{2}$ accumulated as distinct bubbles as a layer between the brine and oil, then passed as a connected chain through the oil. Contrary to the way bubbles pass through the aqueous phase one by one, bubbles passed through the oil in a string (see Fig. 1-25). This was probably to aid in maintaining aqueous lamellae around each $\mathrm{CO}_{2}$ bubble. It was difficult to determine $\mathrm{CO}_{2}$ foam stability in the presence of oil. The $\mathrm{CO}_{2}$ bubbles had irregular shapes and several clear, foam-free areas within the $\mathrm{CO}_{2}$ foam. The content of these clear areas was uncertain, but believed to be dense $\mathrm{CO}_{2}$ because as oil or water it would drain. Even though foam stability decreased with oil present, the oil appeared to impede drainage from the $\mathrm{CO}_{2}$ foam, even while the irregular bubbles coalesced (see Fig. 1-26). Most of the $\mathrm{CO}_{2}$ foam remained in the same structure for at least $90 \mathrm{~min}$ after the end of $\mathrm{CO}_{2}$ injection. Also, note that no obvious large areas without foam formed at the higher concentration of $\mathrm{CD}$ shown in Fig. 1-26. This indicated an increased stability of the $\mathrm{CO}_{2}$ foam at higher concentrations of $\mathrm{CD}$.

\section{Salinity Effect}

Bubble structure and size, gravity drainage, and foam stability were found to be insensitive to salinity for $\mathrm{CD}$ solutions over a wide range of concentrations below and above the CMC, (Figs. 
1-27 and 1-28). This implies that $\mathrm{CD}$ can be used as stable foam over a wide range of field conditions.

\section{Temperature Effect}

Bubble structure and size, gravity drainage, and foam stability under the test conditions were insensitive to temperatures at $60^{\circ} \mathrm{C}$ and below for the concentrations tested, as the three pictures in the top left of Fig. 1-29 show. Tests at $75^{\circ} \mathrm{C}$ saw a marked decrease in stability with rapid decay for the CD 1045 solution concentration of $0.025 \mathrm{wt} \%$; see top right photos in Fig. 1-29. Increasing the surfactant concentration (bottom row of pictures in Fig. 1-29) resulted in increased foam stability. The results imply that the temperature dependence of stability versus CD 1045 concentrations must be considered when preparing a foam system.

\section{Pressure Effect}

Bubble structure and size, gravity drainage, and foam stability changed with pressure at the low surfactant concentration of $0.005 \mathrm{wt} \%$ (first two photos in Fig. 1-28). At surfactant concentrations of $0.025 \mathrm{wt} \%$ and above, the foam system was stable over the testing conditions (second two photos in Fig. 1-30).

In our study, stable foams are usually associated with low IFT. In this study, IFT decreased with pressure increase but foam stability deceased while IFT decreased. This exception shows that low IFT does not always lead to stable foam. According to DLVO theory (Fig. 1-31), the interaction between bubbles changes from repulsion to attraction, which will cause the coalescence of bubbles if the energy barrier can be overcame.

\section{Polymer Effect}

Even thought HPAM can dramatically decrease brine solution's IFT, it does not improve foam generation in brine solutions, which is different from that in surfactants (Fig. 1-32). HPAM can change surface properties but it does not create stable lamellae. While adding in the CD brine solution, HPAM impedes lamellae thinning as mineral oil does, probably due to increase viscosity of the aqueous phase (Fig. 1-33). This generates stable $\mathrm{CO}_{2}$ foam even when $\mathrm{CD} 1045$ is well below its $\mathrm{CMC}$ when HPAM is in the solution. 


\section{pH Effect}

The surface potential and total interaction potential are a function of $\mathrm{pH}$ of the solution. If surface charge is negative, total interaction potential will increase with $\mathrm{pH}$ increase, which results in positive total interaction potential and form stable foam. ${ }^{31}$ When $\mathrm{CD}$ concentration is as low as $0.005 \mathrm{wt} \%$, foam stability decreased with $\mathrm{pH}$ decreases (Figs. 1-34 and 1-35). But when $\mathrm{CD}$ concentration is $0.05 \mathrm{wt} \%$ and above, the foam stability is insensitive to $\mathrm{pH}$ increase (Fig. 1-36).

\section{$\mathrm{CO}_{2}$ Foam Stability after Adsorption in the Core ${ }^{32}$}

During coreflood tests most (approximately 80\%) of CD will be adsorbed or trapped in the core (Tables 1-3 and 1-6). Naturally, IFT will increase with the decrease of concentration of CD (Tables 1-4 and 1-7). From our tests, $0.01 \mathrm{wt} \% \mathrm{CD}$ generates foam, but effluent from a coreflood with similar concentrations will not generate any foam (Fig. 1-37; Tables 1-5 and 1-8). Therefore, some mechanisms change the surfactant bulk properties.

Also, a great portion (more than $80 \%$ ) of calcium lignosulfonate (CLS) will be adsorbed or trapped at the core. CLS is a weak foaming agent that cannot generate foam at a concentration of $0.5 \mathrm{wt} \%$. However CLS is a good, inexpensive sacrificial agent. After preflushing or adding CLS into $\mathrm{CD}$ brine solution, the loss of $\mathrm{CD}$ in the core will decrease to approximately $60 \%$ of original CD loss. There are no doubts that CLS can dramatically decrease the adsorption of CD 1045 in the core (Tables 1-3 and 1-6).

Adsorption of CD is insensitive to injection strategy (Tables 1-3 and 1-6). The stability of effluents is improved by injecting a mixture of CD and CLS rather than preflushing CLS followed by $\mathrm{CD}$, especially in the Berea sandstone (Figs. 1-38 and 1-39). According to $\mathrm{CO}_{2}$ foam stability studies, CLS can help low concentrations of CD brine solution to generate foam. From this point of view, a mixture of CLS and CD 1045 brine solution will work better in the reservoir. 


\section{Lignosulfonate Solution Stability}

Precipitants have been observed in lignosulfonate solutions. This is a matter of concern especially if injecting these solutions into low permeability reservoirs where reduced permeability and thus reduced injectivity could occur. Besides, this there is the inconvenience involved in filtering the precipitates before using the lignosulfonates solutions, the possibilities that the chemical structure or composition is changing, and the effect these might have on the absorbance of the lignosulfonates.

These concerns prompted this preliminary study on reduction of precipitation. The precipitates were examined to see whether they were biological or chemical, and different methods of preventing formation of precipitants were tested. The observations were recorded over three weeks.

\section{Procedures}

Identifying the Precipitates. Microbial experiments were performed on samples of sodium and calcium lignosulfonates that had been prepared for a long period of time, hence having these precipitates, to identify the nature of the precipitates. The components were observed under an optical microscope.

Preventing the Precipitates. Two batches of solutions were prepared with each batch containing six pairs of $2 \%$ brine and $5000 \mathrm{ppm}$ lignosulfonate solutions. A pair is made up of sodium lignosulfonate (SLS) and CLS. Six different treatments were administered to each pair of these solutions and all the solutions were set under ambient conditions (room temperature and pressure).

The first batch was exposed to light and the second was kept in complete darkness. The batch exposed to light was observed each day for a total of 15 days and the batch in darkness was left in total darkness and observed only after 21 days elapsed. Below is a summary of the composition and treatment administered to each pair of solutions. Table1-9 shows the various treatment types used in the experiments. 


\section{Conclusions}

The results from microscope observation of the precipitates show that they are bacteria, fungi and some dirt. For the various treatments of the solutions prepared, Table 1-10 summarizes the observations made on the solutions kept under normal room lighting for 15 days. Table 1-11 summarizes the observations of samples that were kept in the dark for 21 days.

The results summarized in Tables 1-10 and 1-11 indicate that the addition of the chemicals formaldehyde and sodium azide achieved the required objective of preventing precipitation. Sterilizing the solutions also prevented the growth of the precipitates. In the petroleum industry, formaldehyde is a common antibacterial applied in the field; therefore, for field application the formaldehyde treatment is the most promising.

The use of ultrasonic vibrations and purging with nitrogen has no effect on the growths obtained in the original solutions. Bleaching, another method considered, simply decolorizes the solution and caused a different kind of precipitate.

Blocking light prevented the growth of the precipitate of SLS and has little effect on CLS..

\section{References}

1. Schramm, L.L.: "Foams: Fundamentals and Applications in the Petroleum Industry," American Chemical Society, Washington, DC (1994).

2. Bond, D.C and Holbrook, O.C.: “Gas Drive Oil Recovery Process," U. S. Patent No. 2 866507 (December 1958).

3. Fried, A.N: "The Foam Dirve Process for Increasing the Recovery of Oil, " Report RI5866, U.S. Bureau of Mines (1961).

4. Heller, J.P. and Taber, J.J: "Mobility Control for $\mathrm{CO}_{2}$ Floods-A Literature Survey," topial report, Contract No. DE-AC21-79MC10689 U.S. DOE, Washington, DC (October 1980).

5. Heller, J.P., Lien, C.L, and Kuntamukkula, M.S.: "Foamlike Dispersions for Mobility Control in $\mathrm{CO}_{2}$ Floods," SPEJ (August 1985) 603; Trans., AIME 279.

6. Marsden, S.S: "Foam in Porous Media," topical report, Contract No. DE-AC0381SF11564 (SUPRI TR-49) U.S. DOE, Washington, DC (May 19806) 
7. $\quad$ Hirasaki, G.J.: "The Steam-Foam Process," JPT (May 1989) 449.

8. Hirsaki, G.J.: "Supplement to SPE 19505, The Steam-Foam Process-Review of SteamFoam Process Mechanisms," paper SPE 19518 available from SPE Book order Dept., Richardson, TX (1989).

9. Lee, H.O., Heller, J.P., and Hoefer, A.M.W.: "Change in. Apparent Viscosity of $\mathrm{CO}_{2}$ Foam with Rock Permeability," SPERE (November 1991) 421-428.

10. Israelachbvili, J. N.: Intermolecular and Surface Forces. Harcourt Brace \& Company, (1991).

11. Harkins, W.D. and Brown, J.J., "The Determination of Surface Tension (Free Surface Energy), and the Weight of Falling Drops: the Surface Tension of Water and Benzene by the Capillary Height Method," Amer.Chem. Soc (1919) 41, 499,.

12. Adamson, A.W.: Physical Chemistry of Surfaces. New York, John Wiley \& Sons (1982).

13. Hansen, F.K. and Rodsrun, G.; J. Coll \& Inter Sci (1991) 141, 1-12.

14. McCaffery, F.G.: "Measurement of Interfacial Tensions and Contact Angles at High Temperature and Pressure," JCPT, (July-Sept. 1972), 26-32.

15. Worthington, A.M.: "On Pendant Drops," Proceedings of the Royal Society (1881) 32, 362.

16. Andreas. J. M., Hansen, E. A., and Tucker, W. B.: "Boundary Tension by Pendant Drops," Journal of Physical Chemistry, 42, 1038, p1001.

17. Hjelmeland, O. S.: "Experimental Investigation of the Effects of Temperature, Pressure, and Crude Oil Composition on Interracial Properties," paper SPE 12124 presented at the 1983 SPE Annual Technical Conference and Exhibition, San Francisco. CA, Oct. 5-8.

18. Prieditis, J. and Paulett, G.S.: " $\mathrm{CO}_{2}$-Foam Mobility Tests at Reservoir Conditions, in San Andres Cores," paper SPE24178 presented at the 1992 SPE/DOE Symposium on Enhanced Oil Recovery, Tulsa, April 22-24.

19. Kuehne, D. L., Frazier, R. H., Cantor, J., and Horn, W.: "Evaluation of Surfactants for $\mathrm{CO}_{2}$ Mobility in Dolomite Reservoirs,” paper SPE 24177 presented at the 1992 SPE/DOE Symposium on Enhanced Oil Recovery, Tulsa, April 22-24.

20. Yaghoobi, H. and Heller, J. P.: "Laboratory Investigation of Parameters Affecting $\mathrm{CO}_{2-}$ Foam Mobility in Sandstone at Reservoir Conditions," paper SPE 29168 presented at the1994 Eastern Regional Conference and Exhibition, Charleston, November 8-10. 
21. Syahputra, E.: "Experimental Evaluation of Lignosulfonate as a Sacrificial Agent in $\mathrm{CO}_{2}$ Foam Flooding,” M.S. Thesis, New Mexico Institute of Mining and Technology (1999).

22. Fones, J.B. and Adamson, A.W.: "Temperature Dependence of Contact Angle and of Interfacial Free Energies in the Aaphthalene-Water-Air System," Journal of Physical Chemistry, (Feb. 1968). 72 (2), 646-650.

23. Poston. S. W.; Ysrael, S., Hossain, A. K.M. S., Montgomery. E. F., and Ramey, H. J.: "The Effect of Temperature on Irreducible Water Saturation and Relative Permeability of Unconsolidated Sands,” SPEJ (June 1970) 171-180.

24. Schramm, L.L., Fisher, D.B., Schurch, S., and Cameron, A.: "A Captive Drop Instrument for Surface or Interracial Tension Measurements at Elevated Temperatures and Pressures," Colloids and Surfaces A: Physical and Engineering Aspects (1995) 94, 145 159.

25. Wang, W.and Gupta, A.: "Investigation of the Effect of Temperature and Pressure on Wettability Using Modified Pendant Drop Method,” paper SPE 30544 presented at the 1995 SPE Annual Technical Conference \& Exhibition, Dallas, TX, Oct. 22-25.

26. Mackay, E.J.; Henderson, D.H.; Tehrani, D.H. and Danesh, A.: "The Importance of Interfacial Tension on Fluid Distribution During Depressurization,” paper SPE 38919.

27. Lakatos, I.; Bauer. K.; Lakatos-Szabo, J.: "Injection of Lean Gases into Light Oil Reservoirs: Interfacial Aspects," paper SPE 56605 presented at the 1999 SPE Annual Technical Conference and Exhibition, Houston, Oct. 3-6.

28. Zhang, H.R.; Bjorkvik, B.J.A.: "Measurement of Flow Properties and Interfacial Tensions for Gas Condensate," paper SPE 59774 presented at the 2000 SPE/CERI Gas Technology Symposium, Calgary, April 3-5.

29. Holt, T.; Vassenden, F.: "Effects of Pressure on Foam Stability; Implications for Foam Screening," paper SPE 35398 presented at the 1996 SPE/DOE Symposium on Improved Oil Recovery, Tulsa, April 21-24 .

30. Grigg, R.B., Tsau, J.-S., and Martin, F.D.: "Cost Reduction and Injectivity improvements for $\mathrm{CO}_{2}$ Foam for Mobility Control," paper SPE 75178 presented at the 2002 SPE/DPE Symposium on Improved Oil Recovery, Tulsa, April 13-17.

31. Vaidya, R.N. and Fogler, H.S.: "Formation damage due to Colloidally Induced Fines Migration," Colloids and Surfaces, 50 (1990) 215-229. 
32. Grigg, R.B, Bai, B. and Liu, Y.: "Competitive Adsorption of a Hybrid Surfactant System onto Five Minerals, Berea Sandstone and Limestone," paper SPE 90612 presented at the 2004 SPE Annual Technical Conference and Exhibition, Houston, Sep. 26-29.

Table 1-1 Typical Properties of CD

\begin{tabular}{|l|l|}
\hline Appearance & Clear amber liquid \\
\hline Composition & $46.7 \%$ active in water \\
\hline $\mathrm{pH}$ & 5.5 \\
\hline Density & $1.07 \mathrm{~cm}^{3} / \mathrm{g}$ \\
\hline Viscosity & $200 \mathrm{mPas}$ \\
\hline Water Solubility & $100 \%$ \\
\hline
\end{tabular}

Table 1-2 Typical Properties of Alcoflood 935

\begin{tabular}{|l|l|}
\hline Appearance & White granular powder \\
\hline Composition & $92-95 \%$ activity \\
\hline Specific gravity & 0.8 \\
\hline Molecular mass & $5,000,000 \mathrm{~g} / \mathrm{mol}$ \\
\hline Degree of Hydrolysis & $10 \%$ \\
\hline Water Solubility & soluble \\
\hline
\end{tabular}

Table 1-3 Adsorptions in the Limestone

\begin{tabular}{|l|c|c|c|c|}
\hline \multirow{2}{*}{ Injection strategy } & \multicolumn{3}{|c|}{ concentration, mg/L } \\
\cline { 2 - 5 } & \multicolumn{2}{|l|}{ Before adsorption } & \multicolumn{2}{l|}{ After adsorption } \\
\cline { 2 - 5 } & CD & CLS & CD & CLS \\
\hline $500 \mathrm{mg} / \mathrm{L} \mathrm{CD}$ & 500 & & 108 & \\
\hline $5000 \mathrm{mg} / \mathrm{L} \mathrm{CLS}$ & & 5000 & & 3693 \\
\hline Preflush $5000 \mathrm{mg} / \mathrm{L}$ CLS then $500 \mathrm{mg} / \mathrm{L} \mathrm{CD}$ & 500 & 5000 & 244 & \\
\hline Mixture: $5000 \mathrm{mg} / \mathrm{L}$ CLS and $500 \mathrm{mg} / \mathrm{L} \mathrm{CD}$ & 500 & 5000 & 233 & 4024 \\
\hline
\end{tabular}

Table1-4 IFT Values before and after Adsorption in the Limestone

\begin{tabular}{|l|l|c|c|c|}
\hline \multirow{2}{*}{ Injection strategy } & \multicolumn{3}{l|}{ IFT, mN/m } \\
\cline { 2 - 5 } & \multicolumn{2}{|l|}{ Before adsorption } & \multicolumn{2}{l|}{ After adsorption } \\
\cline { 2 - 5 } & CD & CLS & CD & CLS \\
\hline $500 \mathrm{mg} / \mathrm{L} \mathrm{CD}$ & 4.83121 & & 9.54652 & \\
\hline $5000 \mathrm{mg} / \mathrm{L} \mathrm{CLS}$ & & 16.61888 & & \\
\hline Preflush $5000 \mathrm{mg} / \mathrm{L}$ CLS then $500 \mathrm{mg} / \mathrm{L} \mathrm{CD}$ & 4.83121 & 16.61888 & 19.9189 \\
\hline Mixture: $5000 \mathrm{mg} / \mathrm{L}$ CLS and $500 \mathrm{mg} / \mathrm{L} \mathrm{CD}$ & \multicolumn{2}{|c|}{7.2304} & \multicolumn{2}{|c|}{15.7540} \\
\hline
\end{tabular}


Table 1-5 Stability before and after Adsorption in the Limestone

\begin{tabular}{|c|c|c|c|c|}
\hline \multirow{3}{*}{ Injection strategy } & \multicolumn{4}{|l|}{ IFT, $\mathrm{mN} / \mathrm{m}$} \\
\hline & \multicolumn{2}{|c|}{ Before adsorption } & \multicolumn{2}{|c|}{$\begin{array}{l}\text { After } \\
\text { adsorption }\end{array}$} \\
\hline & $\mathrm{CD}$ & CLS & $\mathrm{CD}$ & CLS \\
\hline $500 \mathrm{mg} / \mathrm{L} \mathrm{CD}$ & Stable foam & & No foam & \\
\hline $5000 \mathrm{mg} / \mathrm{L} \mathrm{CLS}$ & & No foam & & \\
\hline Preflush $5000 \mathrm{mg} / \mathrm{L}$ CLS then $500 \mathrm{mg} / \mathrm{L} \mathrm{CD}$ & Stable foam & No foam & \multicolumn{2}{|c|}{ No foam } \\
\hline Mixture: $5000 \mathrm{mg} / \mathrm{L} \mathrm{CLS}$ and $500 \mathrm{mg} / \mathrm{L} \mathrm{CD}$ & \multicolumn{2}{|c|}{ Stable foam } & Margin & oam \\
\hline
\end{tabular}

Table 1-6 Adsorptions in the Berea Sandstone

\begin{tabular}{|l|c|c|c|c|}
\hline \multirow{2}{*}{ Injection strategy } & \multicolumn{3}{|l|}{ concentration, mg/L } \\
\cline { 2 - 5 } & \multicolumn{2}{|l|}{ Before adsorption } & \multicolumn{2}{l|}{ After adsorption } \\
\cline { 2 - 5 } & CD & CLS & CD & CLS \\
\hline $500 \mathrm{mg} / \mathrm{L} \mathrm{CD}$ & 500 & & 81 & \\
\hline $5000 \mathrm{mg} / \mathrm{L}$ CLS & & 5000 & & 3793 \\
\hline Preflush $5000 \mathrm{mg} / \mathrm{L}$ CLS then $500 \mathrm{mg} / \mathrm{L} \mathrm{CD}$ & 500 & 5000 & 212 & 1389 \\
\hline Mixture: $5000 \mathrm{mg} / \mathrm{L}$ CLS and $500 \mathrm{mg} / \mathrm{L}$ CD & 500 & 5000 & 209 & 4289 \\
\hline
\end{tabular}

Table 1-7 IFT before and after Adsorption in the Berea Sandstone

\begin{tabular}{|l|c|c|c|c|}
\hline \multirow{2}{*}{ Injection strategy } & \multicolumn{3}{|l|}{ IFT, $\mathrm{mN} / \mathrm{m}$} \\
\cline { 2 - 5 } & \multicolumn{2}{|l|}{ Before adsorption } & \multicolumn{2}{l|}{ After adsorption } \\
\cline { 2 - 5 } & CD & CLS & CD & CLS \\
\hline $500 \mathrm{mg} / \mathrm{L}$ & 4.83121 & & 9.63746 & \\
\hline $5000 \mathrm{mg} / \mathrm{L}$ CLS & & 16.61888 & & \\
\hline Preflush $5000 \mathrm{mg} / \mathrm{L}$ CLS then $500 \mathrm{mg} / \mathrm{L} \mathrm{CD}$ & 4.83121 & 16.61888 & 18.1783 \\
\hline Mixture: $5000 \mathrm{mg} / \mathrm{L}$ CLS and $500 \mathrm{mg} / \mathrm{L}$ CD & \multicolumn{2}{|c|}{7.2304} & \multicolumn{2}{|l|}{11.3145} \\
\hline
\end{tabular}

Table 1-8 Stability before and after Adsorption in the Berea Sandstone

\begin{tabular}{|l|c|c|c|c|}
\hline \multirow{2}{*}{ Injection strategy } & \multicolumn{2}{|l|}{ IFT, mN/m } \\
\cline { 2 - 5 } & \multicolumn{2}{|l|}{ Before adsorption } & \multicolumn{2}{l|}{ After adsorption } \\
\cline { 2 - 5 } & CD & CLS & CD & CLS \\
\hline $500 \mathrm{mg} / \mathrm{L} \mathrm{CD}$ & Stable foam & & No foam & \\
\hline $5000 \mathrm{mg} / \mathrm{L} \mathrm{CLS}$ & & No foam & & \\
\hline Preflush $5000 \mathrm{mg} / \mathrm{L}$ CLS then $500 \mathrm{mg} / \mathrm{L} \mathrm{CD}$ & Stable foam & No foam & \multicolumn{2}{|c|}{ No foam } \\
\hline Mixture: $5000 \mathrm{mg} / \mathrm{L}$ CLS and $500 \mathrm{mg} / \mathrm{L} \mathrm{CD}$ & \multicolumn{2}{|c|}{ Stable foam } & \multicolumn{2}{|c|}{$50 \%$ foam } \\
\hline
\end{tabular}


Table 1-9 Tests Identifications

\begin{tabular}{|l|l|}
\hline Treatment Type & Composition \\
\hline No treatment (Standard) & $5000 \mathrm{ppm}$ Ligno $+2 \%$ Brine \\
\hline Ultrasonic & $5000 \mathrm{ppm}$ Ligno $+2 \%$ Brine \\
\hline Bleach (sodium hypochlorite) & $1 \%$ bleach $+5000 \mathrm{ppm}$ Ligno $+2 \%$ Brine \\
\hline Sodium azide (Sodium Trinitride) & $0.1 \%$ sodium azide $+5000 \mathrm{ppm}$ Ligno $+2 \%$ Brine \\
\hline Formaldehyde $(\mathrm{HCHO})$ & $1000 \mathrm{ppm}$ HCHO $+5000 \mathrm{ppm}$ Ligno $+2 \%$ Brine \\
\hline Boiling & $5000 \mathrm{ppm}$ Ligno $+2 \%$ Brine \\
\hline
\end{tabular}


Table 1-10 The Results of Tests Exposed to the Light

\begin{tabular}{|c|c|c|c|c|c|c|}
\hline $\begin{array}{l}\text { TREATMENT } \\
\text { TYPE }\end{array}$ & $\begin{array}{l}\text { SAMPLE } \\
\text { TYPE }\end{array}$ & DAY 1 & DAY 2 & DAY 3 & DAY 4 & DAY 5 \\
\hline \multirow[t]{2}{*}{$\begin{array}{c}\text { STANDARD } \\
\text { (NO TREATMENT) }\end{array}$} & Calcium & None & $\begin{array}{l}\text { Few strands } \\
\text { formed }\end{array}$ & Strands increase & $\begin{array}{c}\text { Strands } \\
\text { entangle to } \\
\text { form a mesh }\end{array}$ & Mesh grows \\
\hline & Sodium & None & $\begin{array}{l}\text { Few strands } \\
\text { formed }\end{array}$ & Strands increase & $\begin{array}{l}\text { Strands increase } \\
\text { and precipitate } \\
\text { forms }\end{array}$ & Mesh formed \\
\hline \multirow{2}{*}{$\begin{array}{c}0.1 \% \\
\text { FORMALDEHYDE }\end{array}$} & Calcium & \multicolumn{5}{|c|}{ None } \\
\hline & Sodium & \multicolumn{5}{|c|}{ None } \\
\hline \multirow{2}{*}{$\begin{array}{c}0.1 \% \text { SODIUM } \\
\text { AZIDE }\end{array}$} & Calcium & \multicolumn{5}{|c|}{ None } \\
\hline & Sodium & \multicolumn{5}{|c|}{ None } \\
\hline \multirow{2}{*}{$\begin{array}{l}\text { ULTRASONIC + } \\
\text { NITROGEN }\end{array}$} & Calcium & None & $\begin{array}{l}\text { Few strands } \\
\text { formed }\end{array}$ & Strands increase & Mesh formed & $\begin{array}{c}\text { Mesh becomes } \\
\text { jelly-like }\end{array}$ \\
\hline & Sodium & None & $\begin{array}{l}\text { Few strands } \\
\text { formed }\end{array}$ & Strands increase & Mesh formed & $\begin{array}{l}\text { Mesh becomes } \\
\text { jelly-like }\end{array}$ \\
\hline \multirow[t]{2}{*}{$\begin{array}{c}1 \% \text { SODIUM } \\
\text { HYPOCHLORITE }\end{array}$} & Calcium & $\begin{array}{c}\text { Solution was } \\
\text { decolorized } \\
\text { and a } \\
\text { precipitate } \\
\text { was formed } \\
\end{array}$ & \multicolumn{4}{|c|}{ No change after 1st day } \\
\hline & Sodium & $\begin{array}{c}\text { Solution was } \\
\text { decolorized } \\
\text { and a } \\
\text { precipitate } \\
\text { was formed }\end{array}$ & \multicolumn{4}{|c|}{ No change after 1st day } \\
\hline \multirow{2}{*}{$\begin{array}{c}\text { STERILIZED } \\
\text { (BOILED \& } \\
\text { FILTERED) }\end{array}$} & Calcium & \multicolumn{5}{|c|}{ None } \\
\hline & Sodium & \multicolumn{5}{|c|}{ None } \\
\hline
\end{tabular}


Table 1-10. The Results of Tests Exposed to the Light (cont.)

\begin{tabular}{|c|c|c|c|c|c|c|}
\hline $\begin{array}{l}\text { TREATMENT } \\
\text { TYPE }\end{array}$ & $\begin{array}{l}\text { SAMPLE } \\
\text { TYPE }\end{array}$ & DAY 6 & DAY 7 & DAY 8 & DAY 9 & DAY 10 \\
\hline \multirow{2}{*}{$\begin{array}{l}\text { STANDARD } \\
\text { (NO TREATMENT) }\end{array}$} & Calcium & $\begin{array}{l}\text { Mesh } \\
\text { becomes } \\
\text { jelly-like }\end{array}$ & Increase & Increase & Increase & Increase \\
\hline & Sodium & $\begin{array}{l}\text { Mesh } \\
\text { becomes } \\
\text { jelly-like }\end{array}$ & Increase & Increase & Increase & Increase \\
\hline \multirow{2}{*}{$\begin{array}{l}0.1 \% \\
\text { FORMALDEHYDE }\end{array}$} & Calcium & \multicolumn{5}{|c|}{ None } \\
\hline & Sodium & \multicolumn{5}{|c|}{ None } \\
\hline \multirow{2}{*}{$\begin{array}{l}0.1 \% \text { SODIUM } \\
\text { AZIDE }\end{array}$} & Calcium & \multicolumn{5}{|c|}{ None } \\
\hline & Sodium & \multicolumn{5}{|c|}{ None } \\
\hline \multirow{2}{*}{$\begin{array}{l}\text { ULTRASONIC + } \\
\text { NITROGEN }\end{array}$} & Calcium & $\begin{array}{l}\text { Increase } \\
\text { (highest } \\
\text { growth) }\end{array}$ & Increase & \multicolumn{3}{|c|}{ No change after $7^{\text {th }}$ day } \\
\hline & Sodium & $\begin{array}{l}\text { Increase } \\
\text { (highest } \\
\text { growth) }\end{array}$ & Increase & \multicolumn{3}{|c|}{ No change after $7^{\text {th }}$ day } \\
\hline \multirow[t]{2}{*}{$\begin{array}{l}1 \% \text { SODIUM } \\
\text { HYPOCHLORITE }\end{array}$} & Calcium & \multicolumn{5}{|c|}{ No change after $1^{\text {st }}$ day } \\
\hline & Sodium & \multicolumn{5}{|c|}{ No change after $1^{\text {st }}$ day } \\
\hline \multirow{2}{*}{$\begin{array}{l}\text { STERILIZED } \\
\text { (BOILED \& } \\
\text { FILTERED) }\end{array}$} & Calcium & \multicolumn{5}{|c|}{ None } \\
\hline & Sodium & \multicolumn{5}{|c|}{ None } \\
\hline
\end{tabular}


Table 1-10. The Results of Tests Exposed to the Light (cont.)

\begin{tabular}{|c|c|c|c|c|c|c|}
\hline $\begin{array}{l}\text { TREATMENT } \\
\text { TYPE }\end{array}$ & $\begin{array}{l}\text { SAMPLE } \\
\text { TYPE }\end{array}$ & DAY 11 & DAY 12 & DAY 13 & DAY 14 & DAY 15 \\
\hline \multirow{2}{*}{$\begin{array}{l}\text { STANDARD } \\
\text { (NO TREATMENT) }\end{array}$} & Calcium & \multicolumn{5}{|c|}{ No change after $10^{\text {th }}$ day } \\
\hline & Sodium & \multicolumn{5}{|c|}{ No change after $10^{\text {th }}$ day } \\
\hline \multirow{2}{*}{$\begin{array}{l}0.1 \% \\
\text { FORMALDEHYDE }\end{array}$} & Calcium & \multicolumn{5}{|c|}{ None } \\
\hline & Sodium & \multicolumn{5}{|c|}{ None } \\
\hline \multirow{2}{*}{$\begin{array}{l}0.1 \% \text { SODIUM } \\
\text { AZIDE }\end{array}$} & Calcium & \multicolumn{5}{|c|}{ None } \\
\hline & Sodium & \multicolumn{5}{|c|}{ None } \\
\hline \multirow{2}{*}{$\begin{array}{l}\text { ULTRASONIC + } \\
\text { NITROGEN }\end{array}$} & Calcium & \multicolumn{5}{|c|}{ No change after $7^{\text {th }}$ day } \\
\hline & Sodium & \multicolumn{5}{|c|}{ No change after $7^{\text {th }}$ day } \\
\hline \multirow{2}{*}{$\begin{array}{l}1 \% \text { SODIUM } \\
\text { HYPOCHLORITE }\end{array}$} & Calcium & \multicolumn{5}{|c|}{ No change after $7^{\text {th }}$ day } \\
\hline & Sodium & \multicolumn{5}{|c|}{ No change after $7^{\text {th }}$ day } \\
\hline \multirow{2}{*}{$\begin{array}{l}\text { STERILIZED } \\
\text { (BOILED \& } \\
\text { FILTERED) }\end{array}$} & Calcium & \multicolumn{5}{|c|}{ None } \\
\hline & Sodium & \multicolumn{5}{|c|}{ None } \\
\hline
\end{tabular}


Table 1-11 The Results of Tests in the Dark

\begin{tabular}{|c|c|c|}
\hline TREATMENT TYPE & $\begin{array}{l}\text { SAMPLE TYPE } \\
\text { (Kept in the dark) }\end{array}$ & Observations after 21 days \\
\hline \multirow{2}{*}{$\begin{array}{c}\text { STANDARD } \\
\text { (NO TREATMENT) }\end{array}$} & Calcium & Jelly like stuff \\
\hline & Sodium & None \\
\hline \multirow[b]{2}{*}{$0.1 \%$ FORMALDEHYDE } & Calcium & None \\
\hline & Sodium & None \\
\hline \multirow{2}{*}{ ULTRASONIC + NITROGEN } & Calcium & Jelly like stuff \\
\hline & Sodium & None \\
\hline \multirow{2}{*}{$1 \%$ SODIUM HYPOCHLORIDE } & Calcium & None \\
\hline & Sodium & None \\
\hline \multirow{2}{*}{$\begin{array}{c}\text { STERILIZED } \\
\text { (BOILED \& FILTERED) }\end{array}$} & Calcium & None \\
\hline & Sodium & None \\
\hline
\end{tabular}




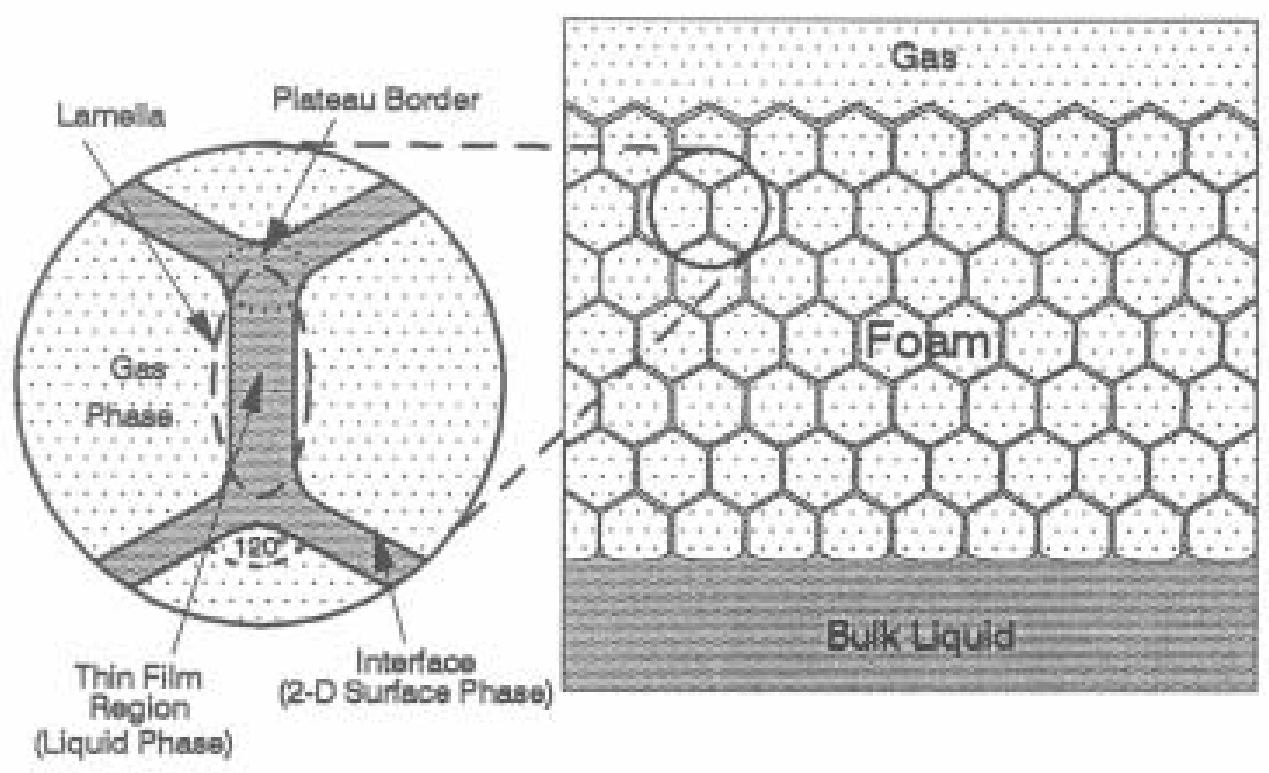

Fig 1-1. A generalized foam system. ${ }^{1}$

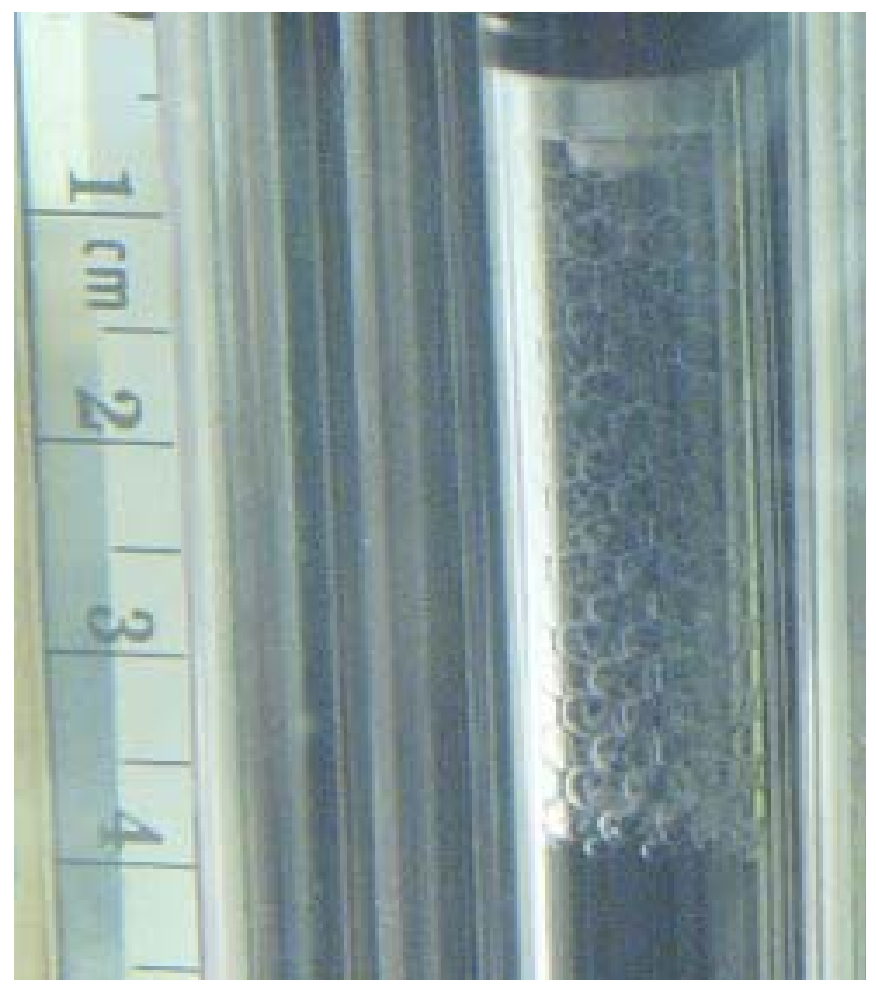

Fig 1-2. Image of $\mathrm{CO}_{2}$ foams in $\mathrm{CD} 0.025 \%$ solution. 


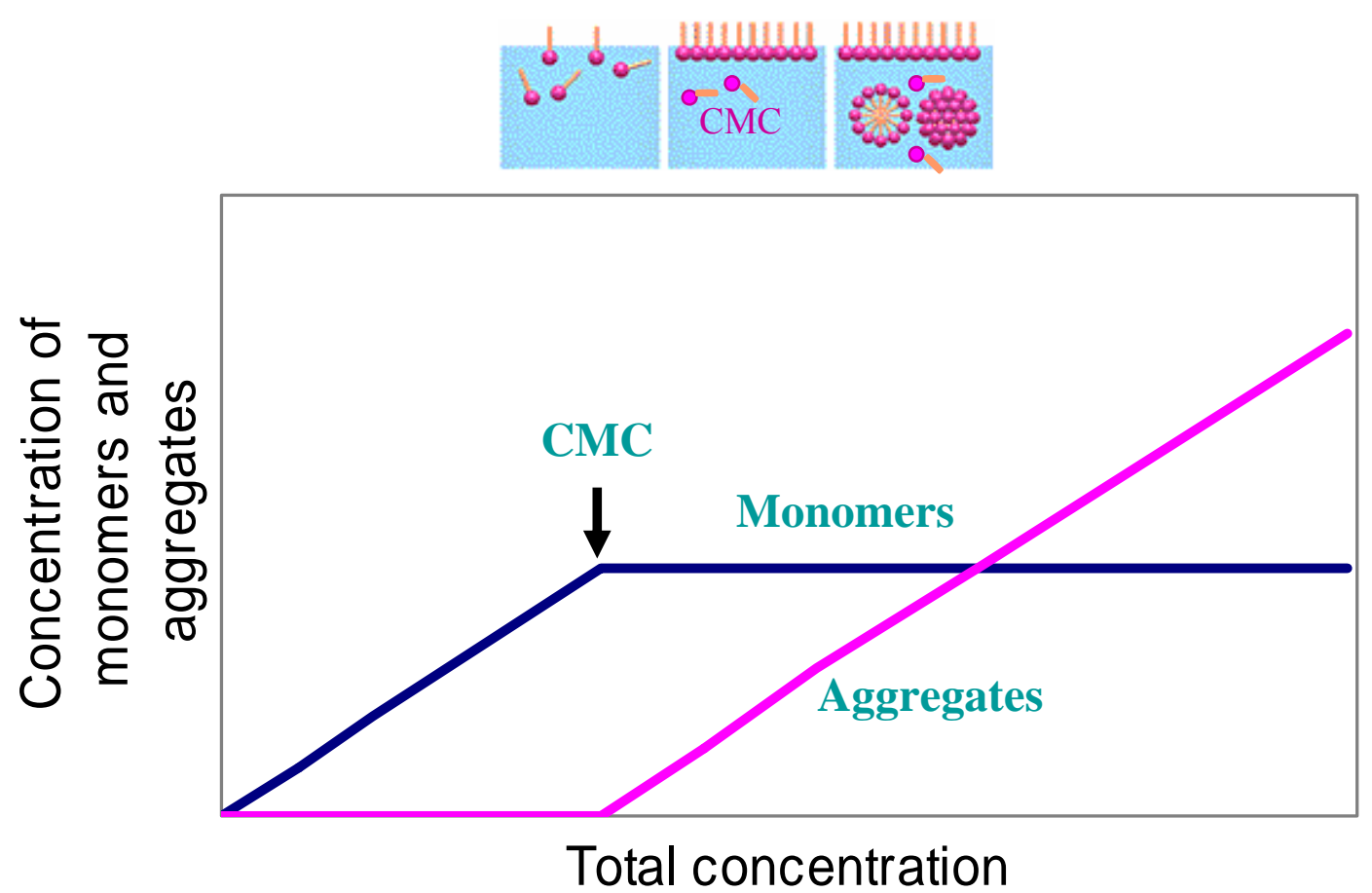

Fig 1-3. Monomer (X1) and aggregate (micelle, $\mathrm{Xn}$ ) concentrations as a function of total concentration. $^{10}$

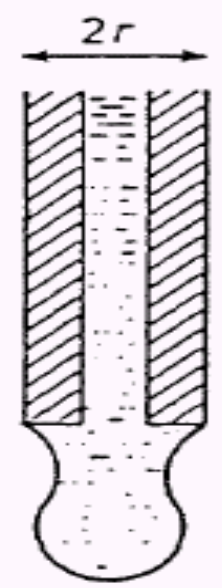

Fig. 1-4. Cartoon of capillary and drop used to determine IFT in the drop weight method. 


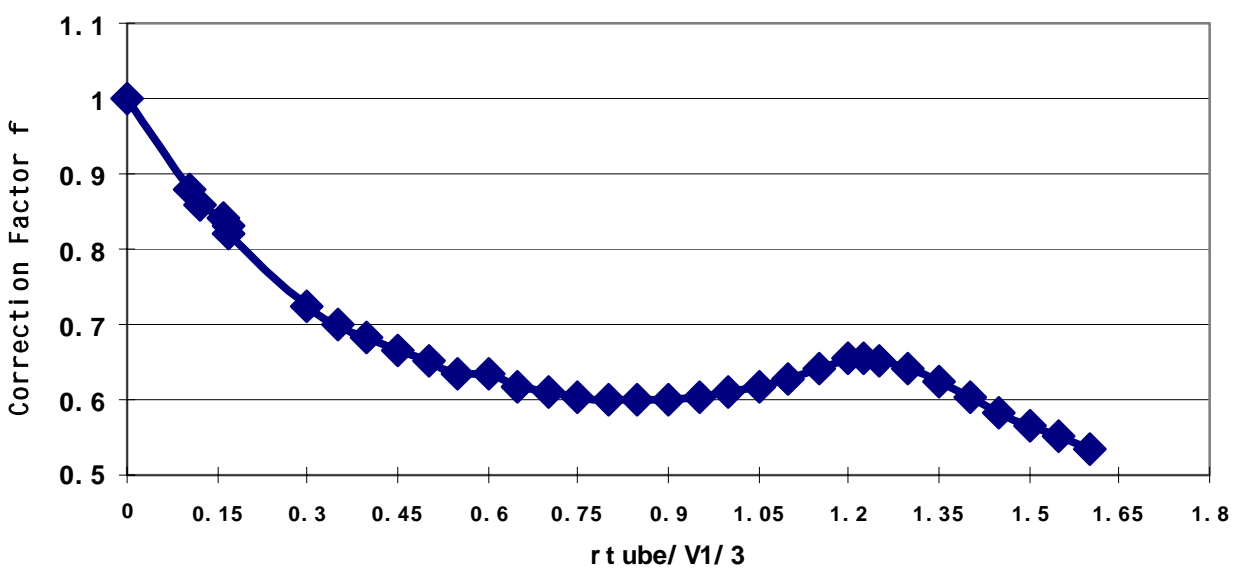

Fig. 1-5. Harkins-Brown correction factor for drop-weight method after Adamson. ${ }^{12}$

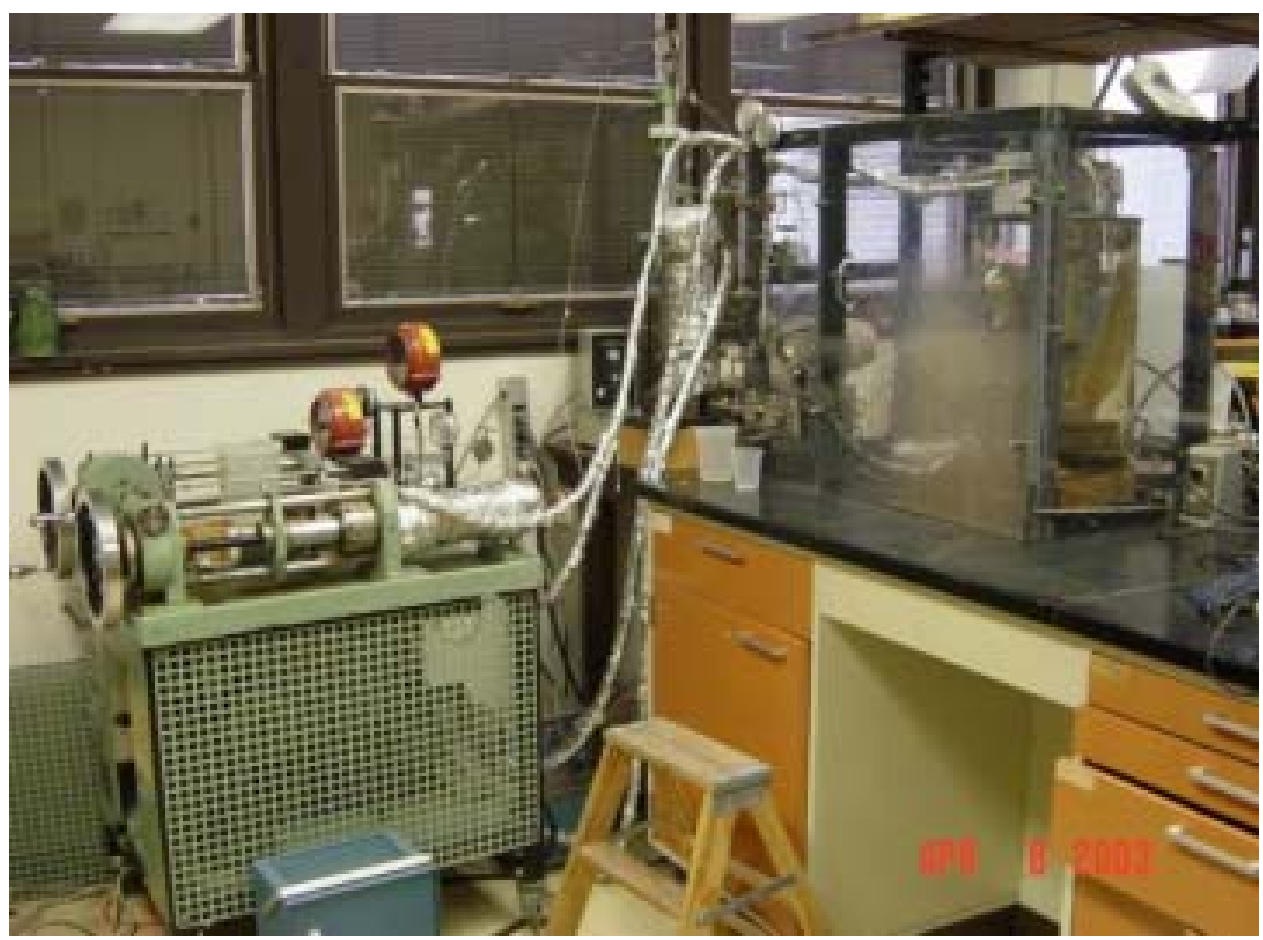

Fig. 1-6. Foam stability apparatus setup. 


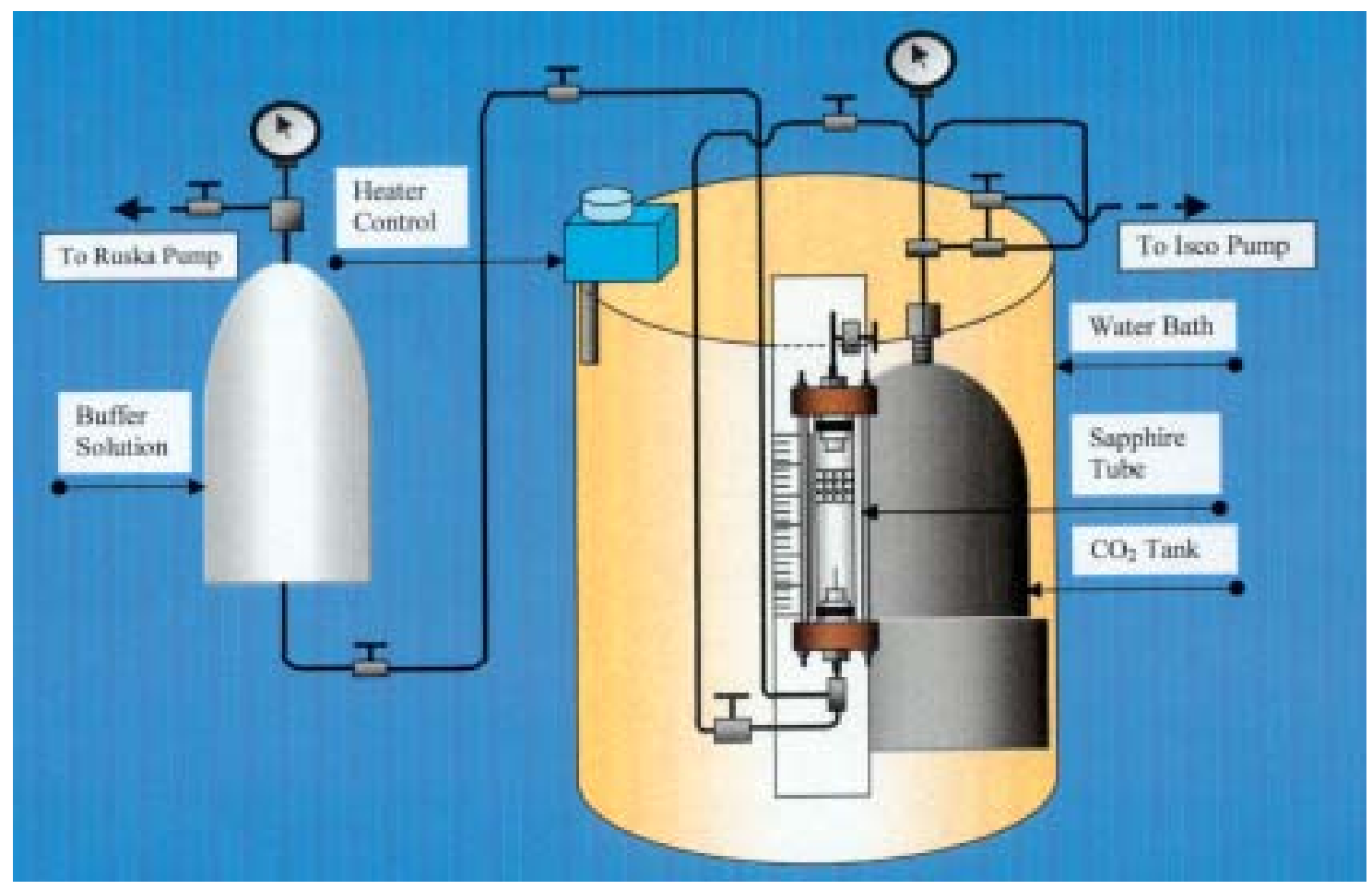

Fig.1-7. Foam stability apparatus.
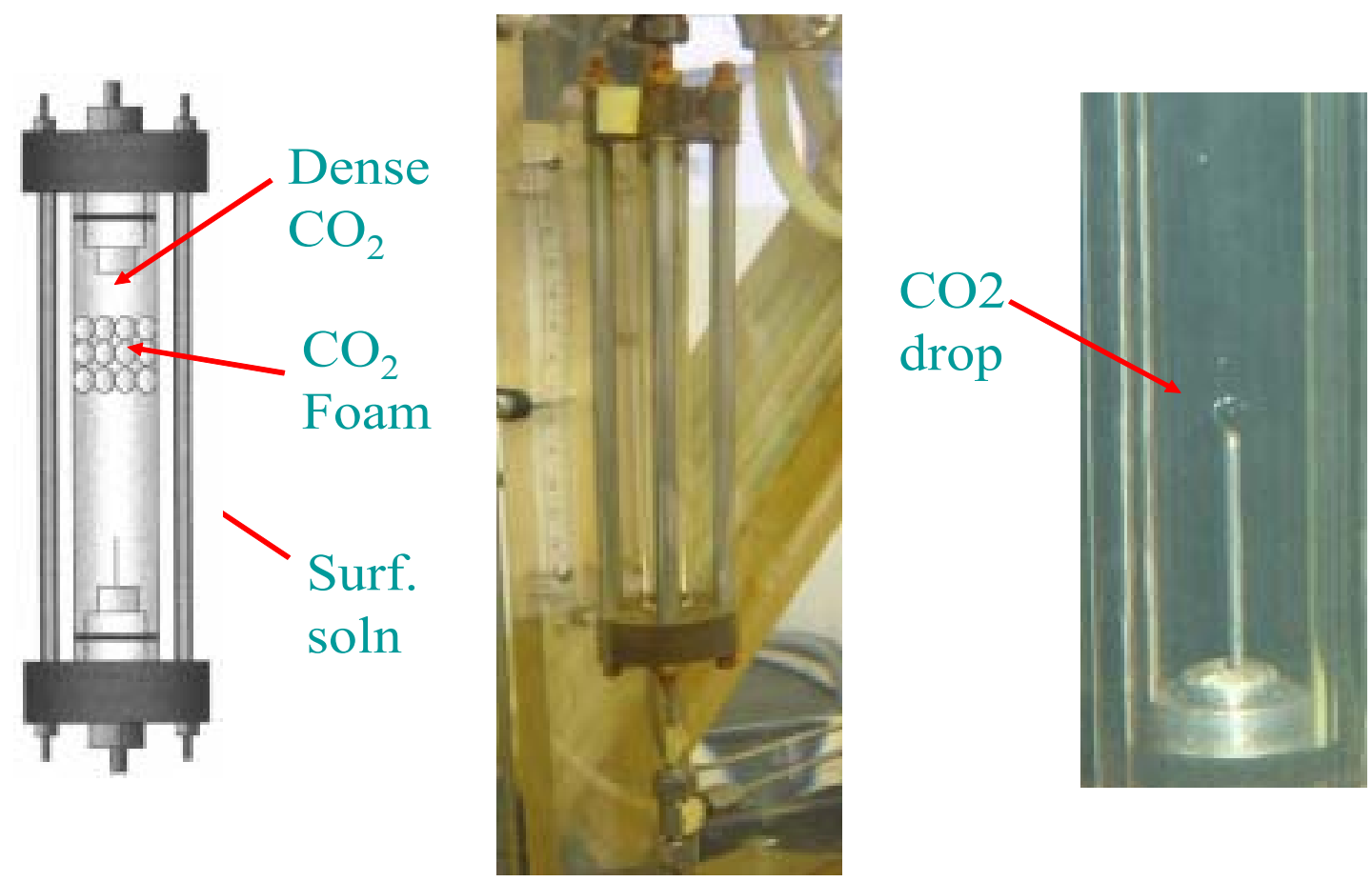

Fig. 1-8. The sapphire tube cell. 


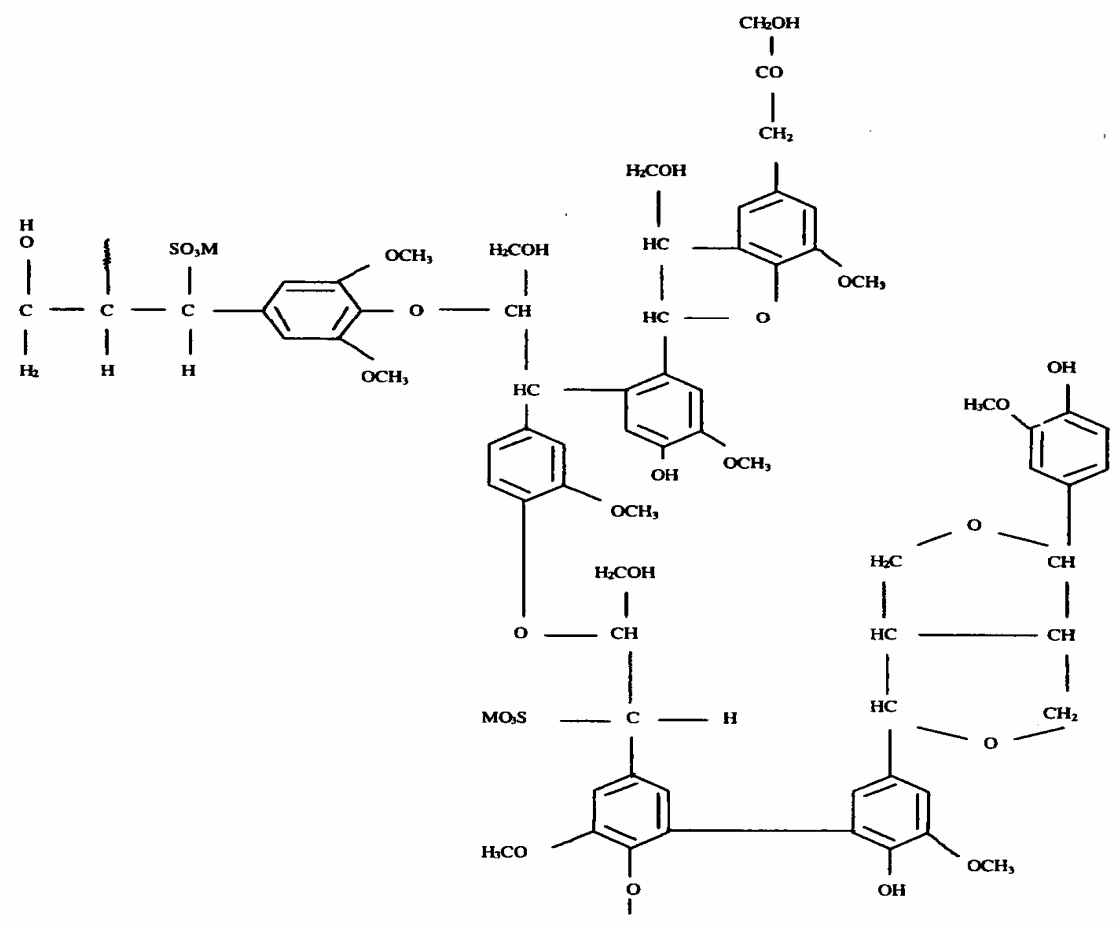

Fig 1-9. Example of a section of lignosulfonate molecule. ${ }^{21}$

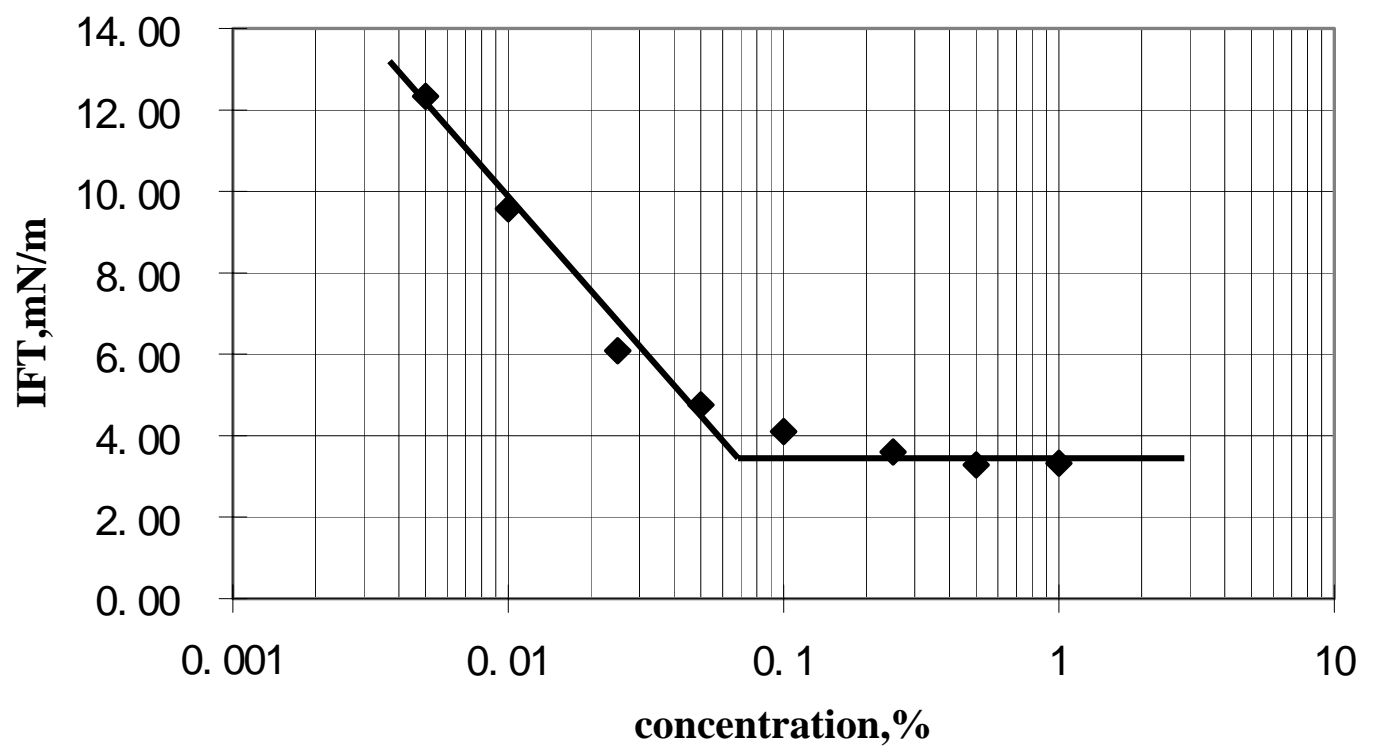

Fig. 1-10. CMC determination for CD. 


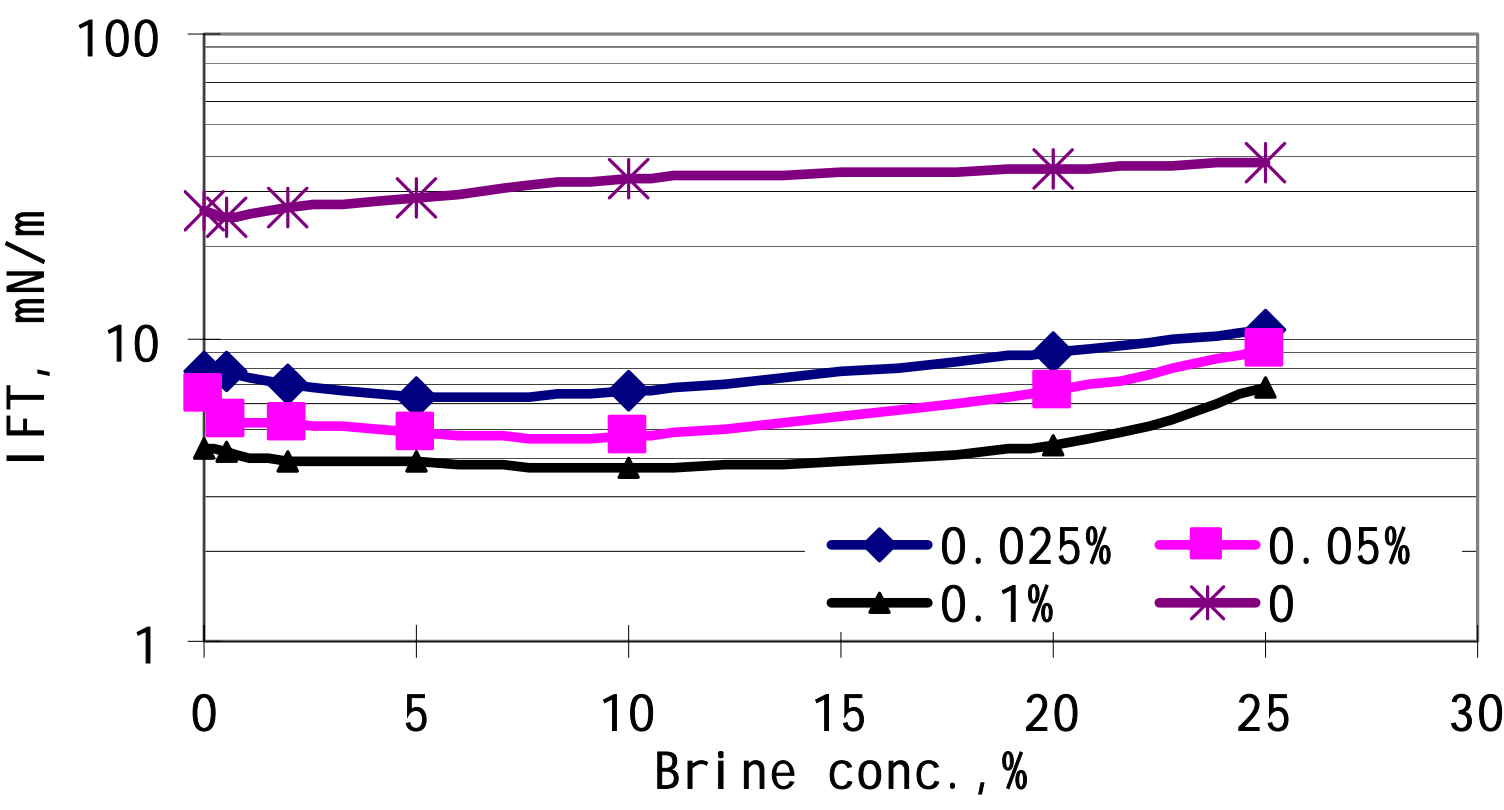

Fig. 1-11 Salinity effect on IFT of CD solutions.

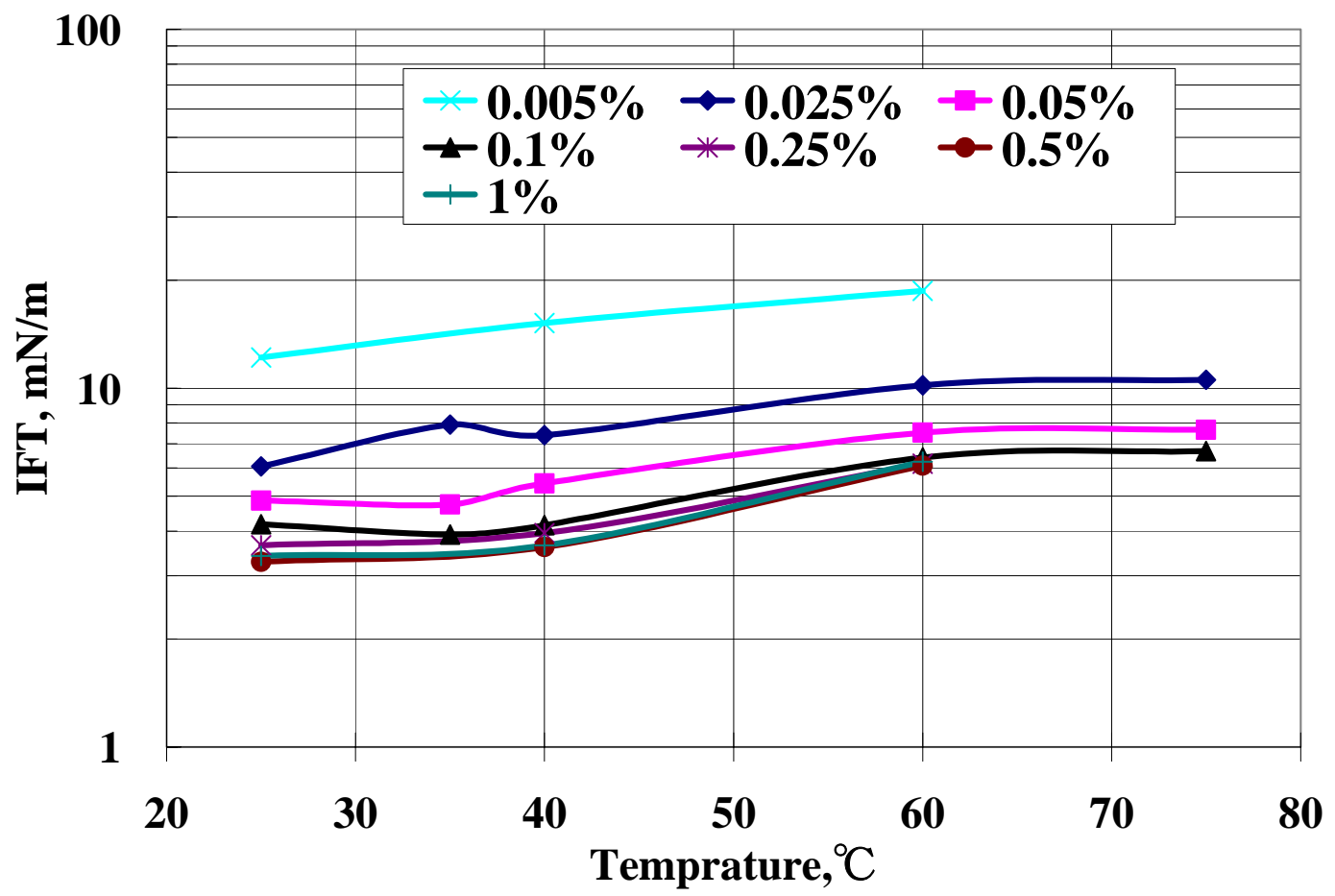

Fig. 1-12. IFT vs. temperature and surfactant concentration in CD solutions. 


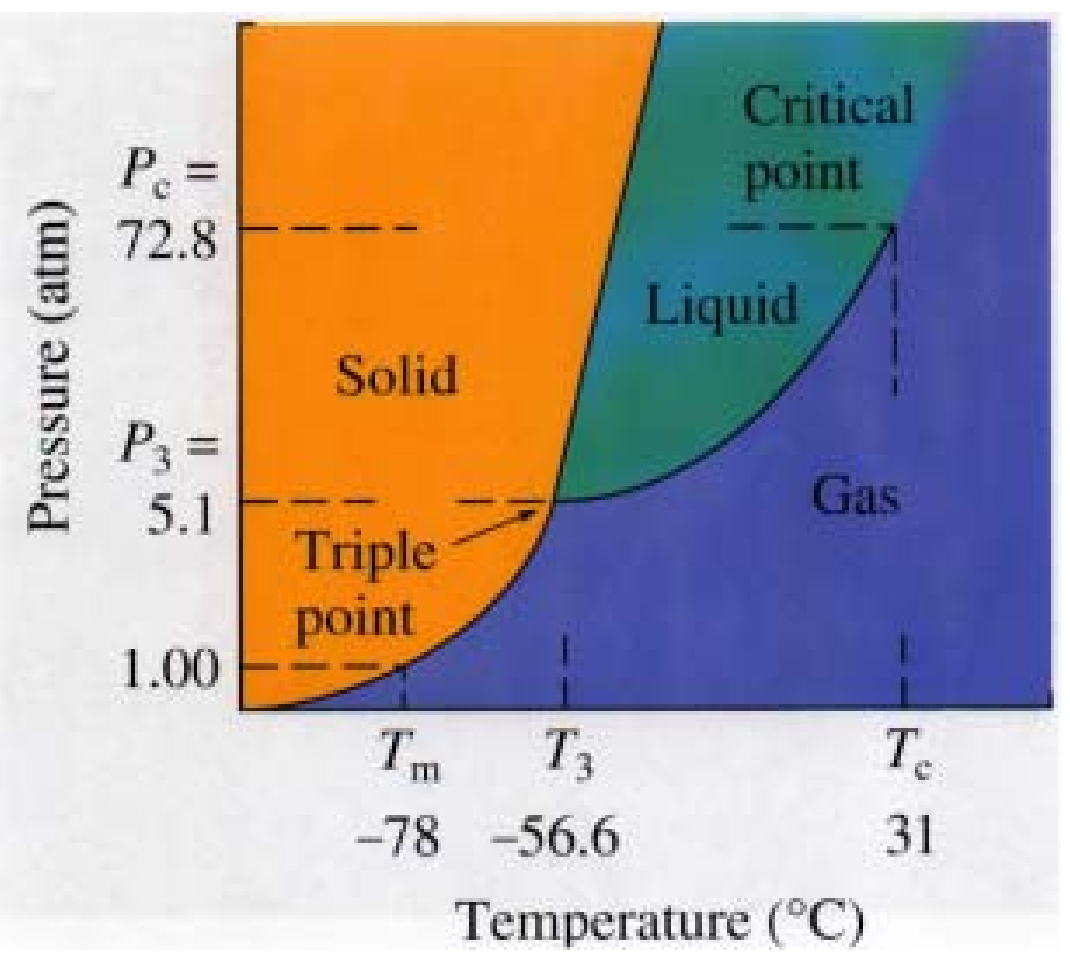

Fig. 1-13. $\mathrm{CO}_{2}$ phase diagram.

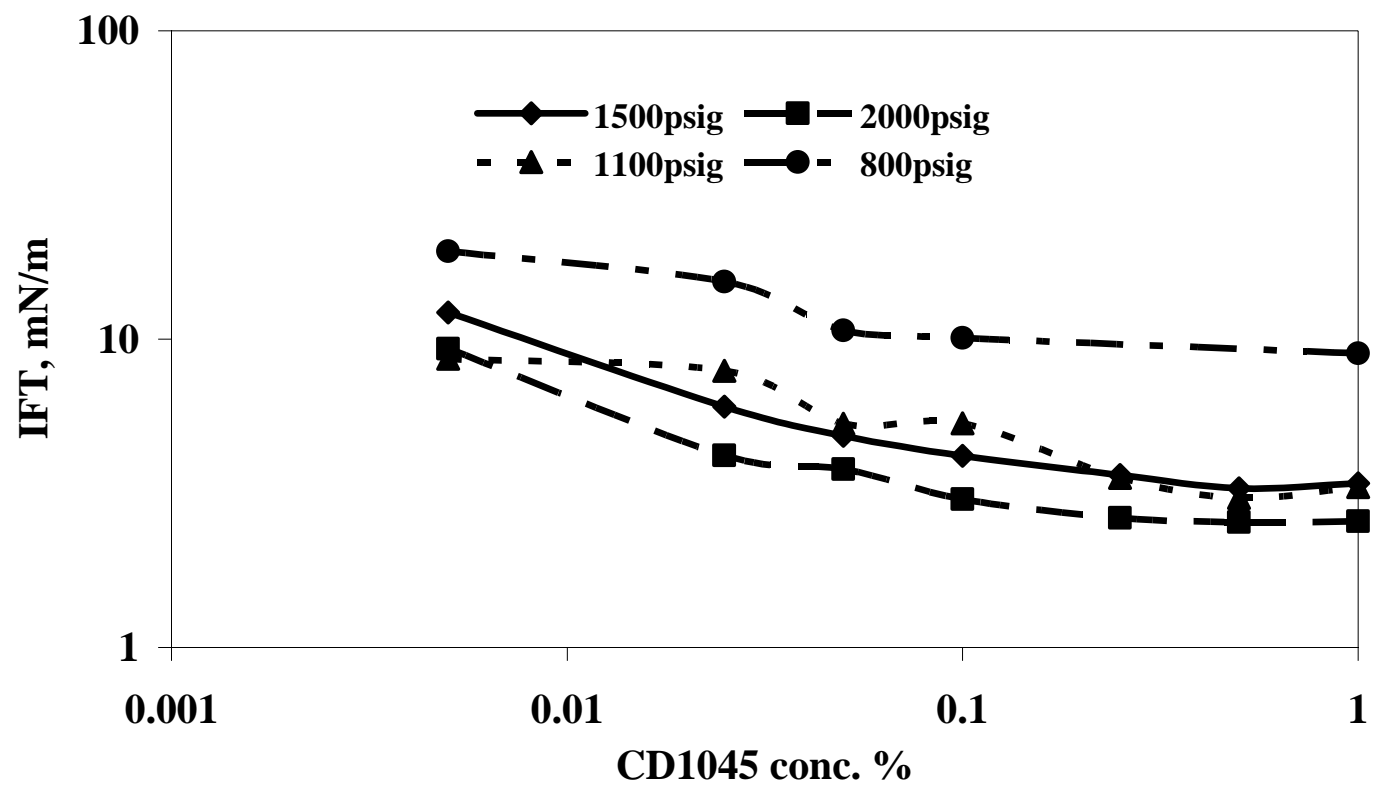

Fig. 1-14. IFT vs. pressure and surfactant concentration. 


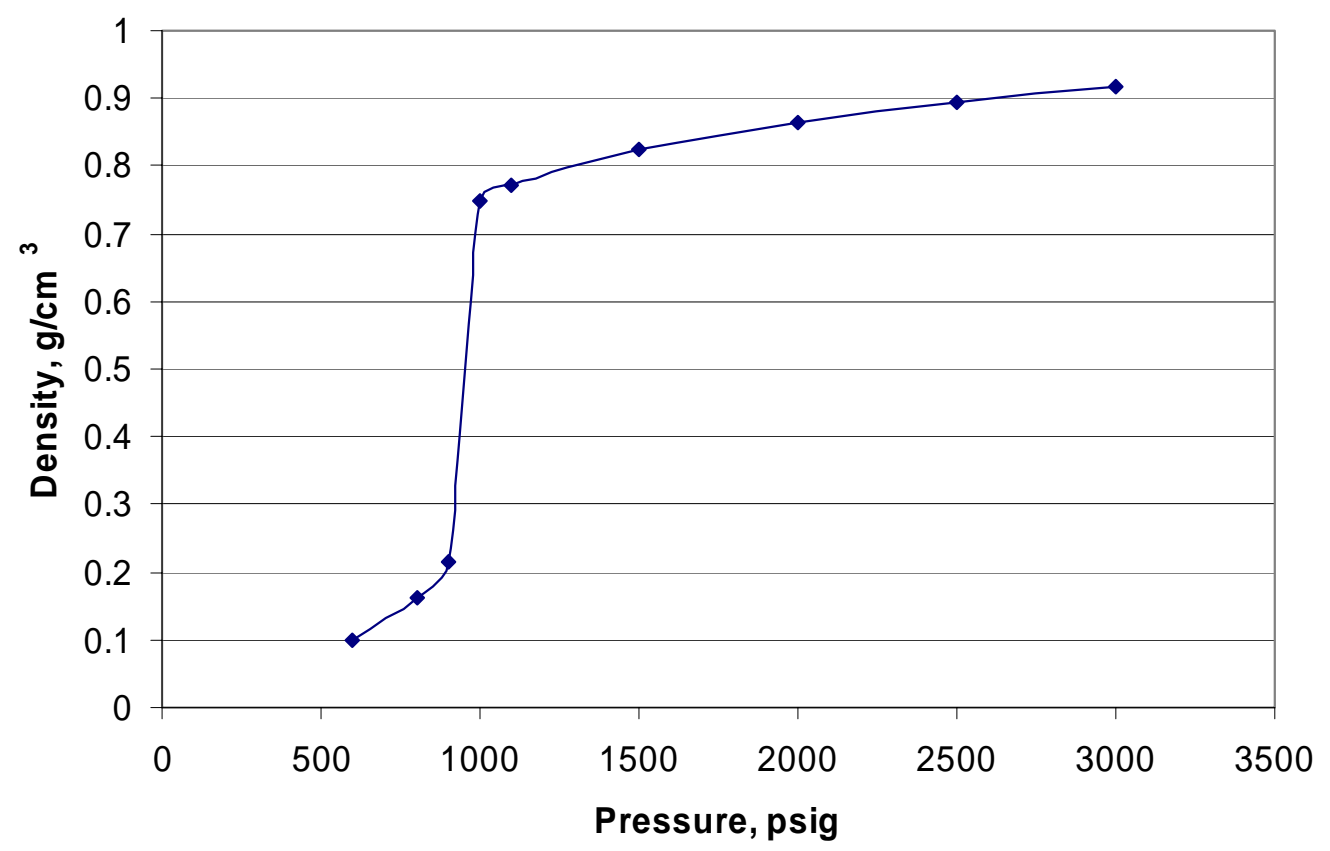

Fig. $1-15 \mathrm{CO}_{2}$ density vs. pressure at $25^{\circ} \mathrm{C}$

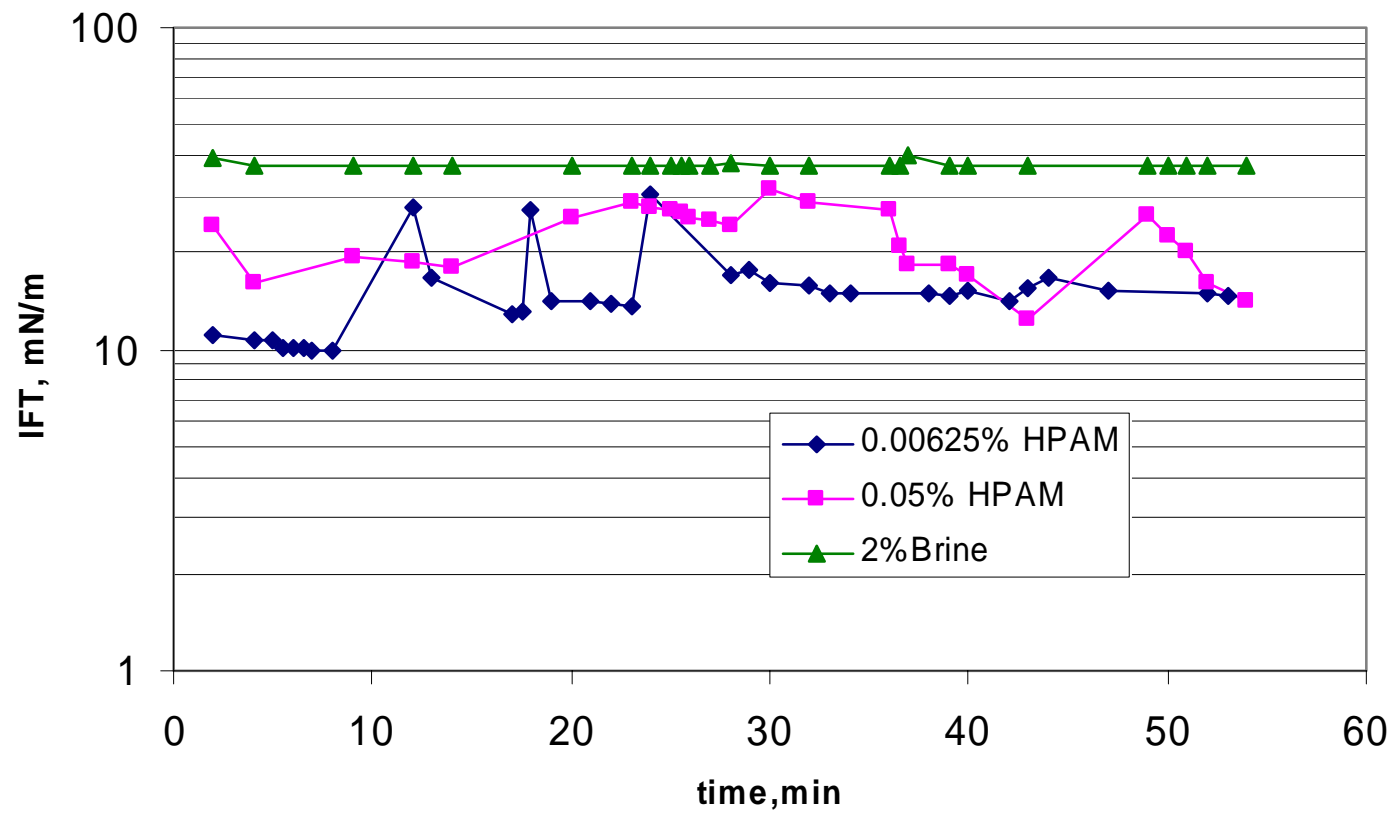

Fig. 1-16. Polymer effect on IFT. 


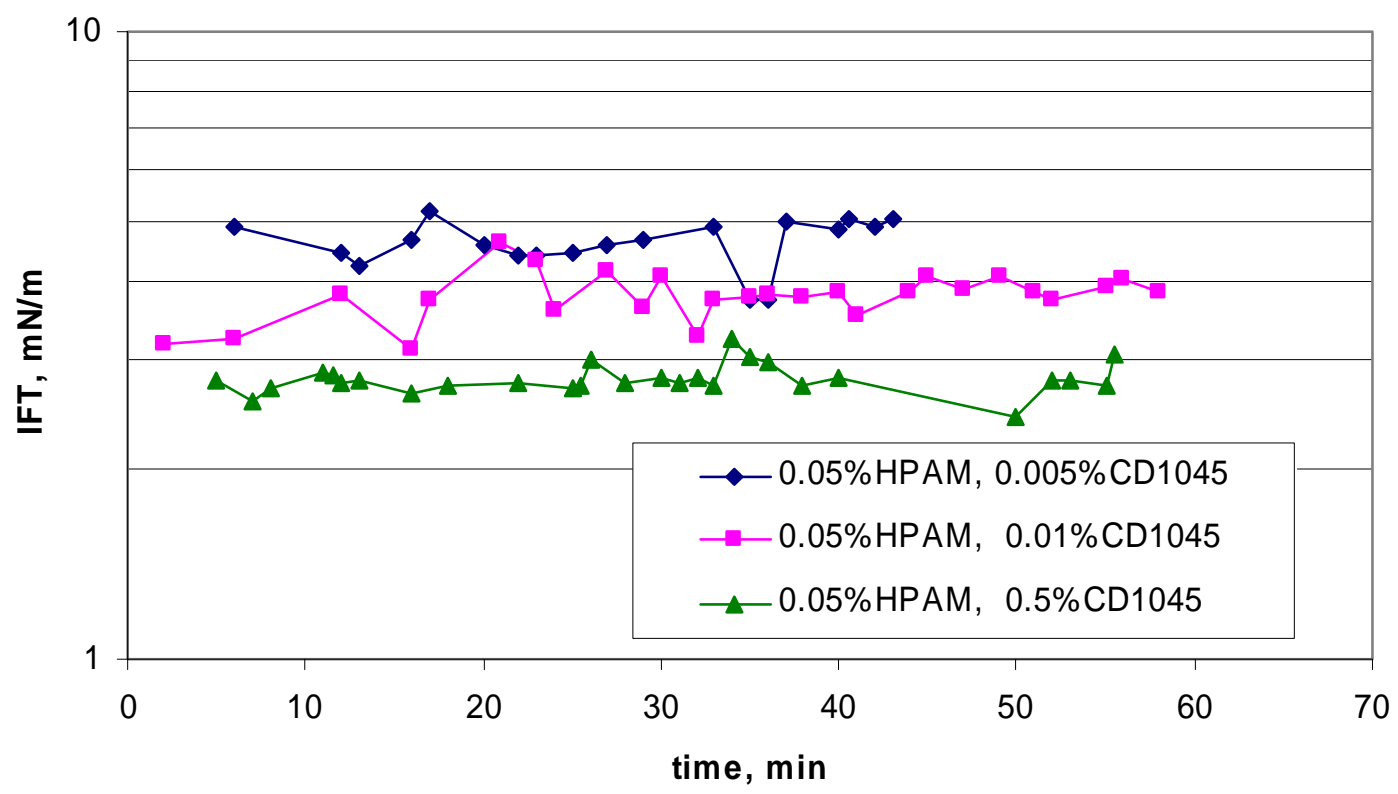

Fig. 1-17. Dynamic IFT for polymer in CD1045 brine solution.

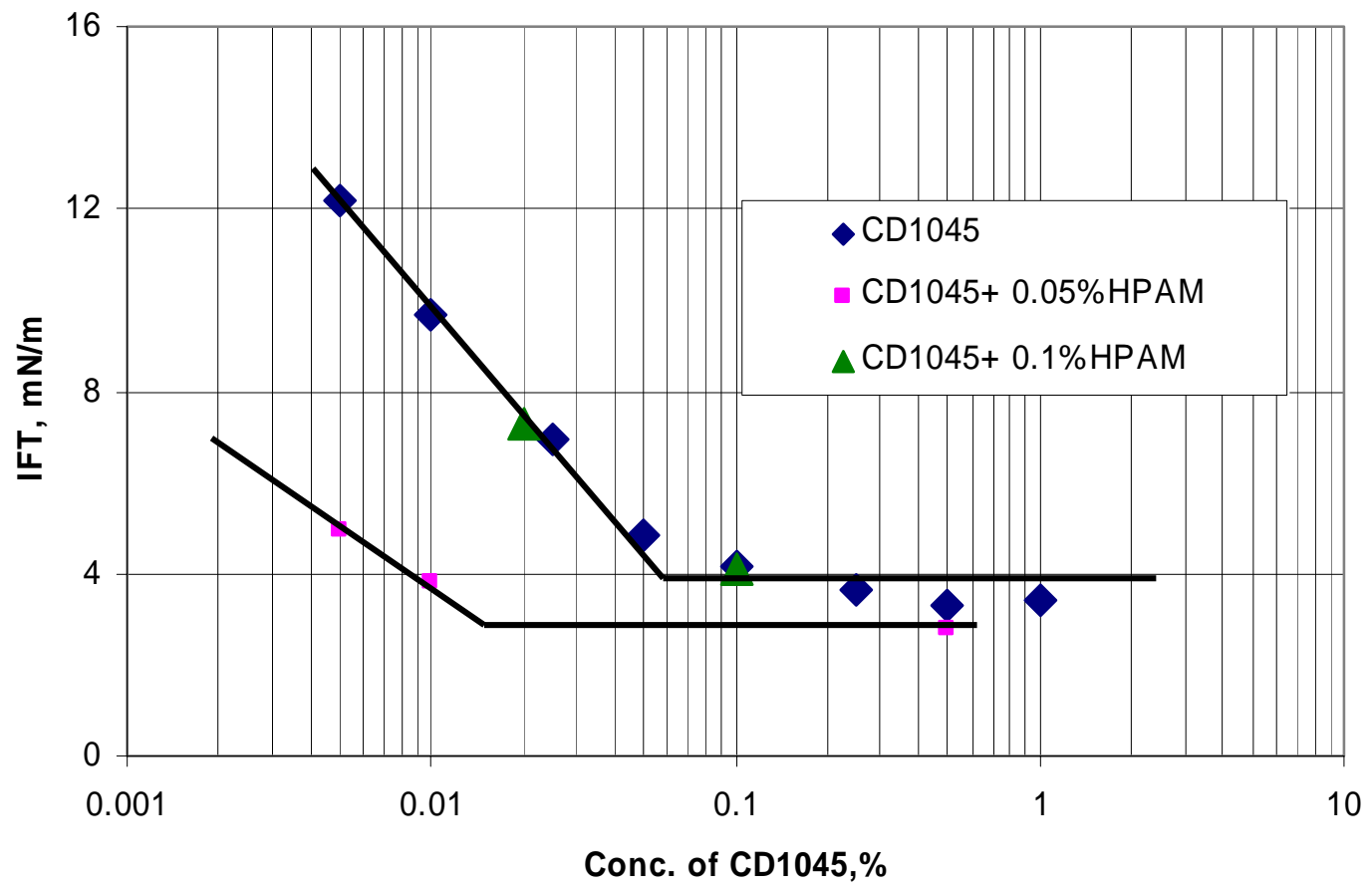

Fig. 1-18. Polymer effect on IFT in CD brine solution. 


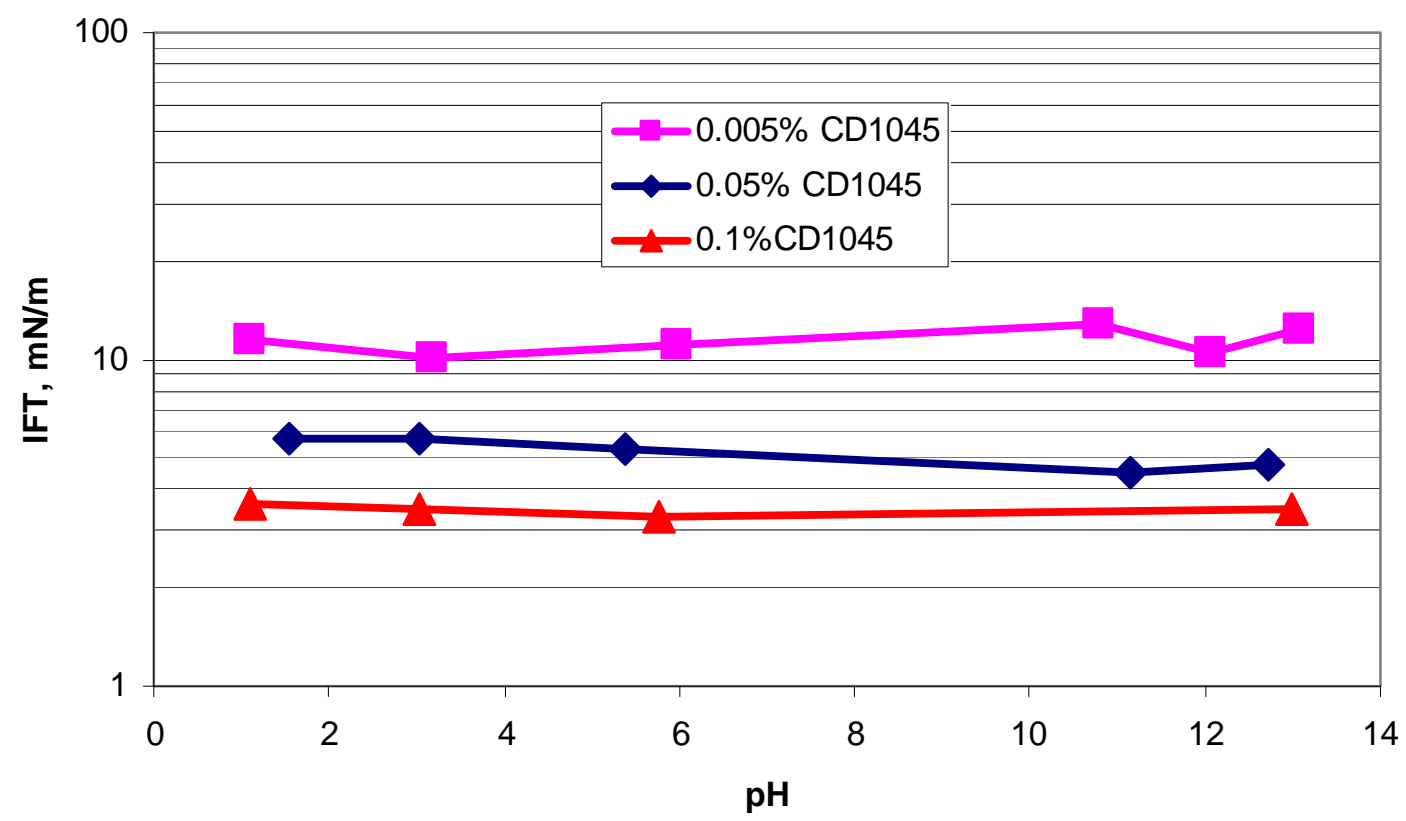

Fig. 1-19. pH effect on IFT for CD in $2 \%$ brine solution.

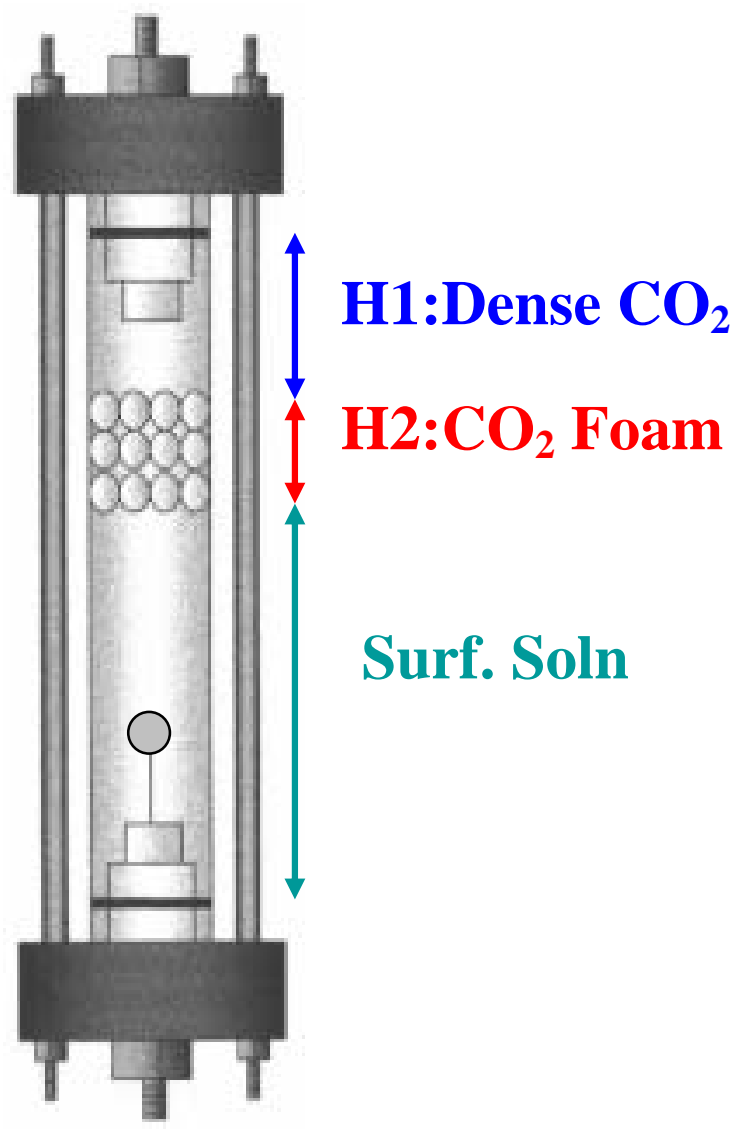

Fig. 1-20. $\mathrm{CO}_{2}$ foam stability test. 


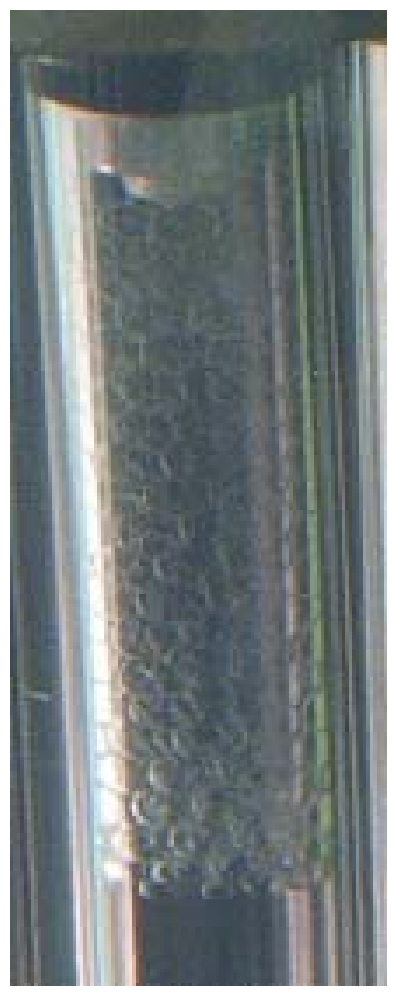

$0.025 \mathrm{wt} \% \mathrm{CD}$

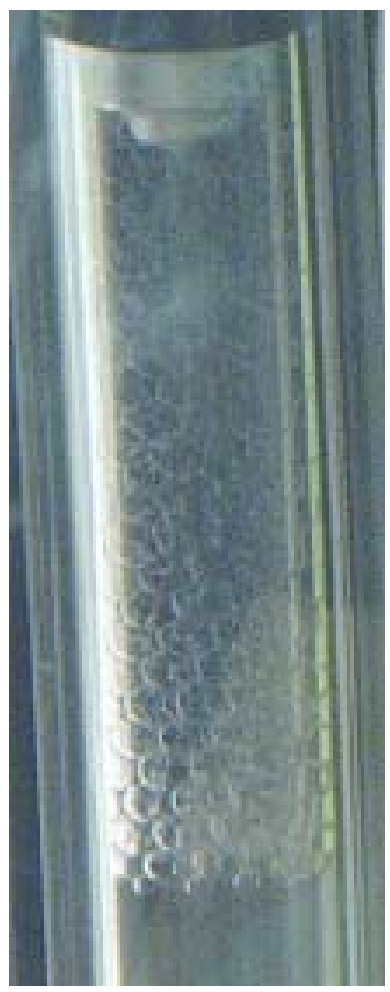

$0.05 \mathrm{wt} \% \mathrm{CD}$

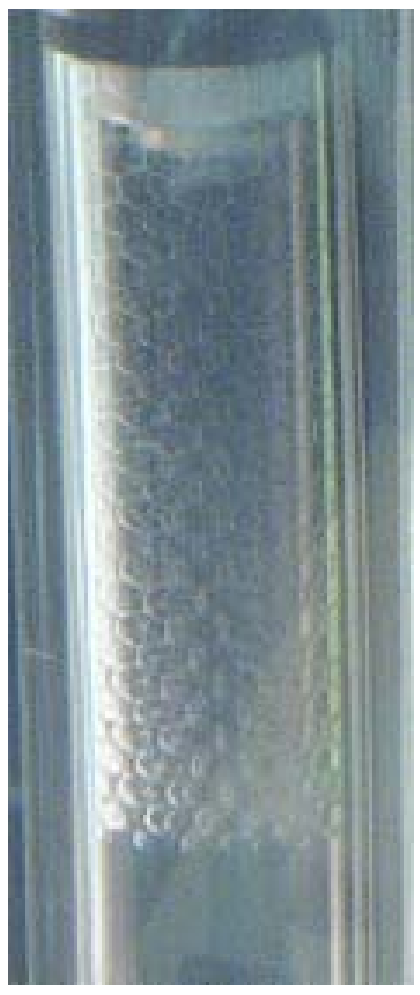

$0.1 \mathrm{wt} \% \mathrm{CD}$

Fig. 1-21. Foam stability at different CD concentrations.

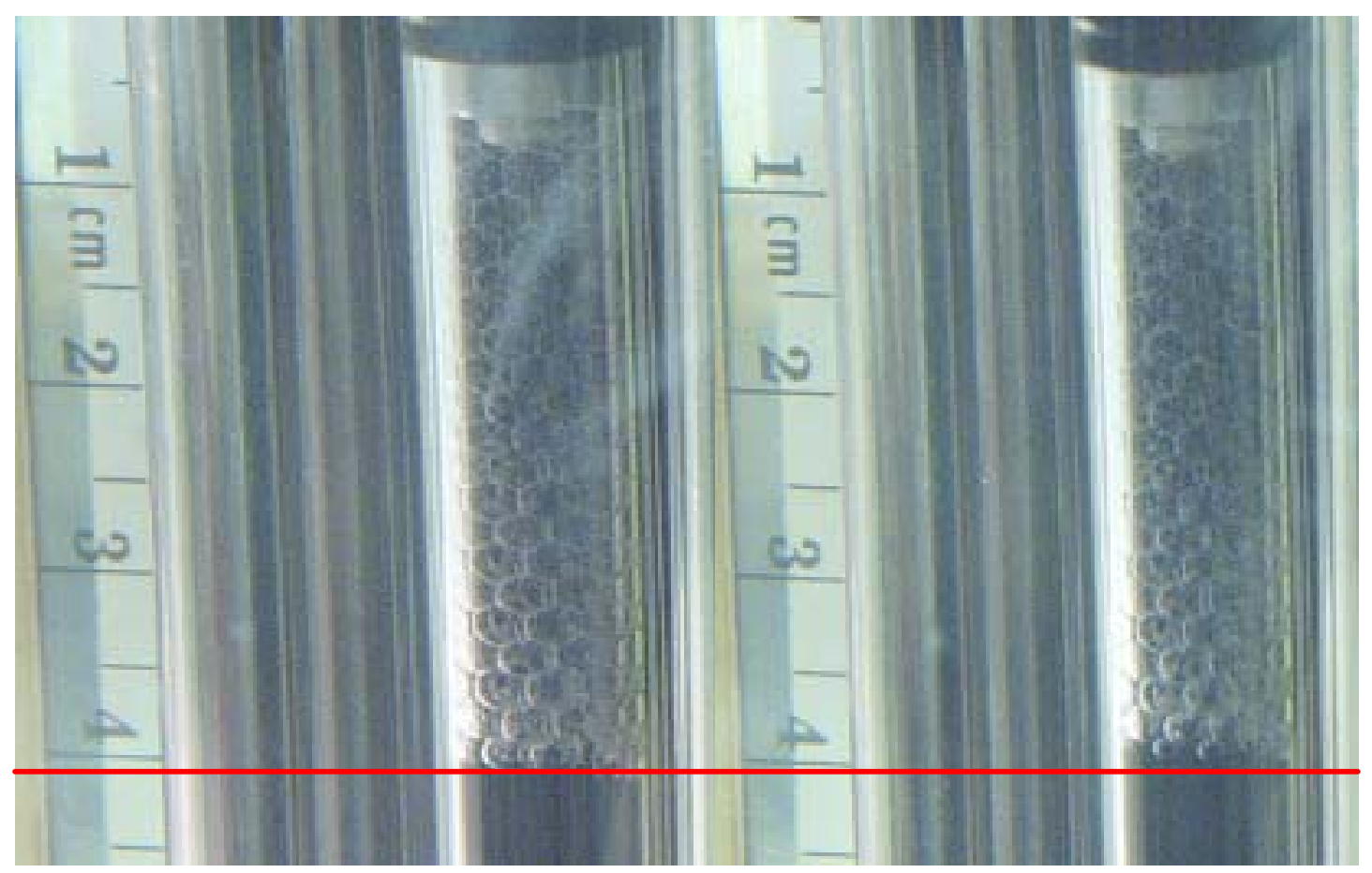

Fig. 1-22. Small volume changes occur with time from gravity drainage even for stable foams. 


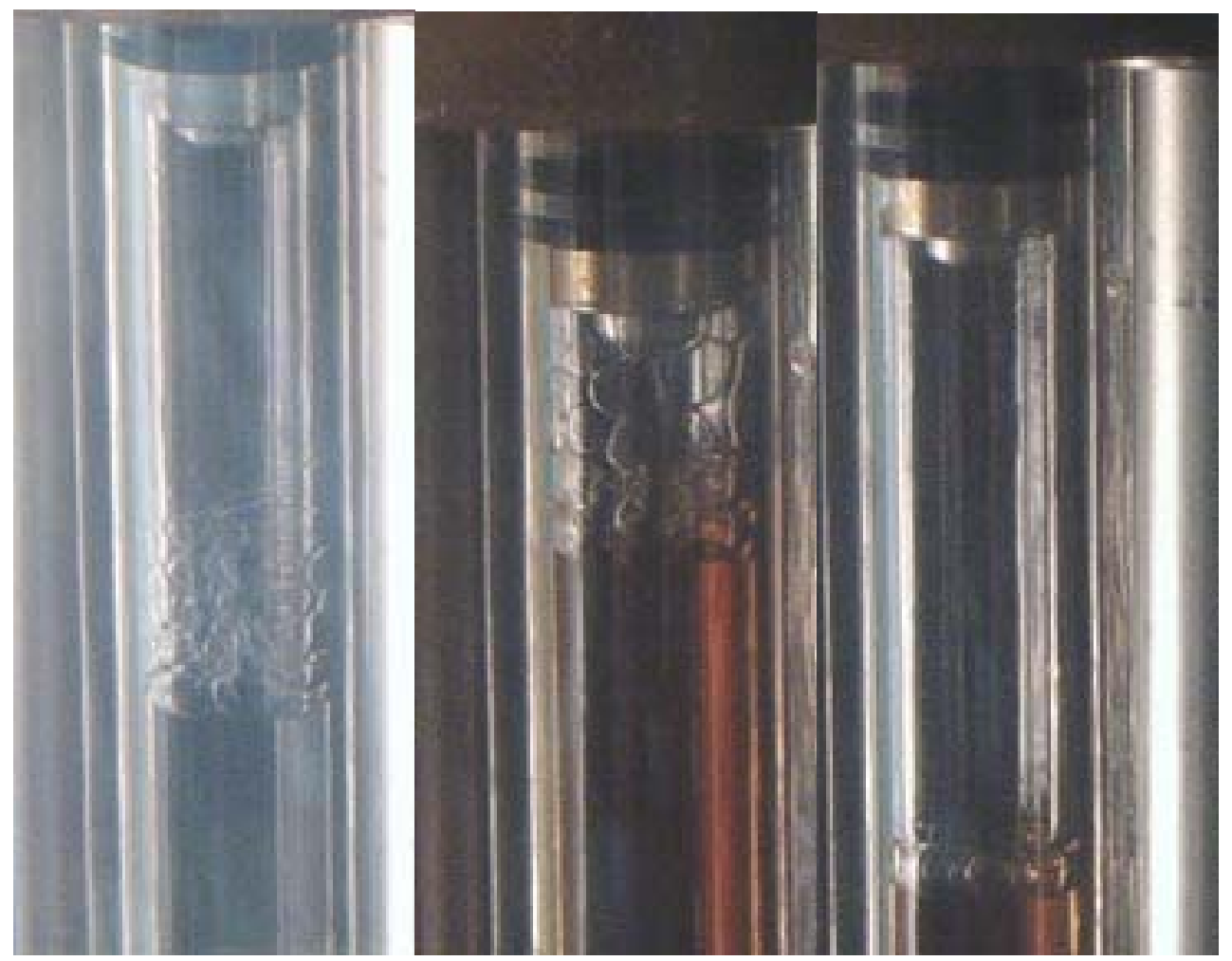

Fig. 1-23. Unstable foam bubbles are neither well defined nor homogeneous shapes.

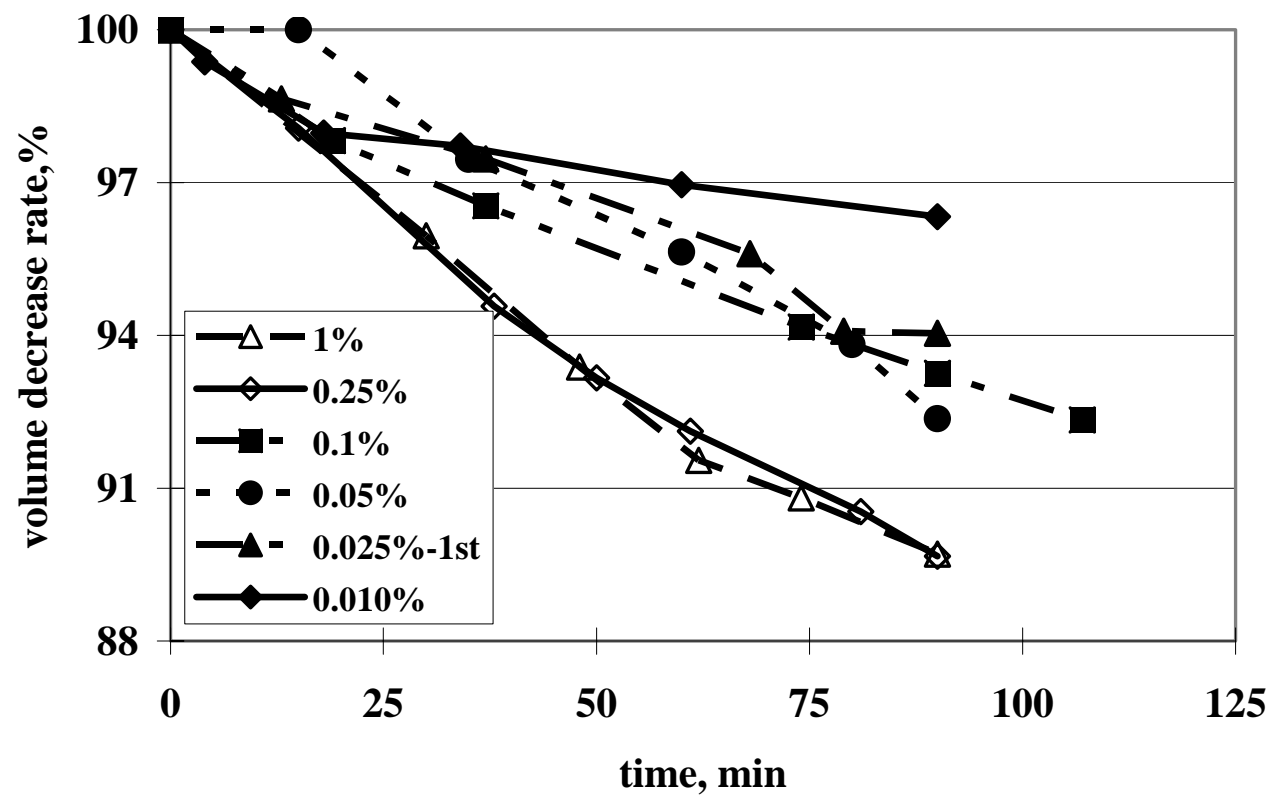

Fig. 1-24. Gravity drainage 

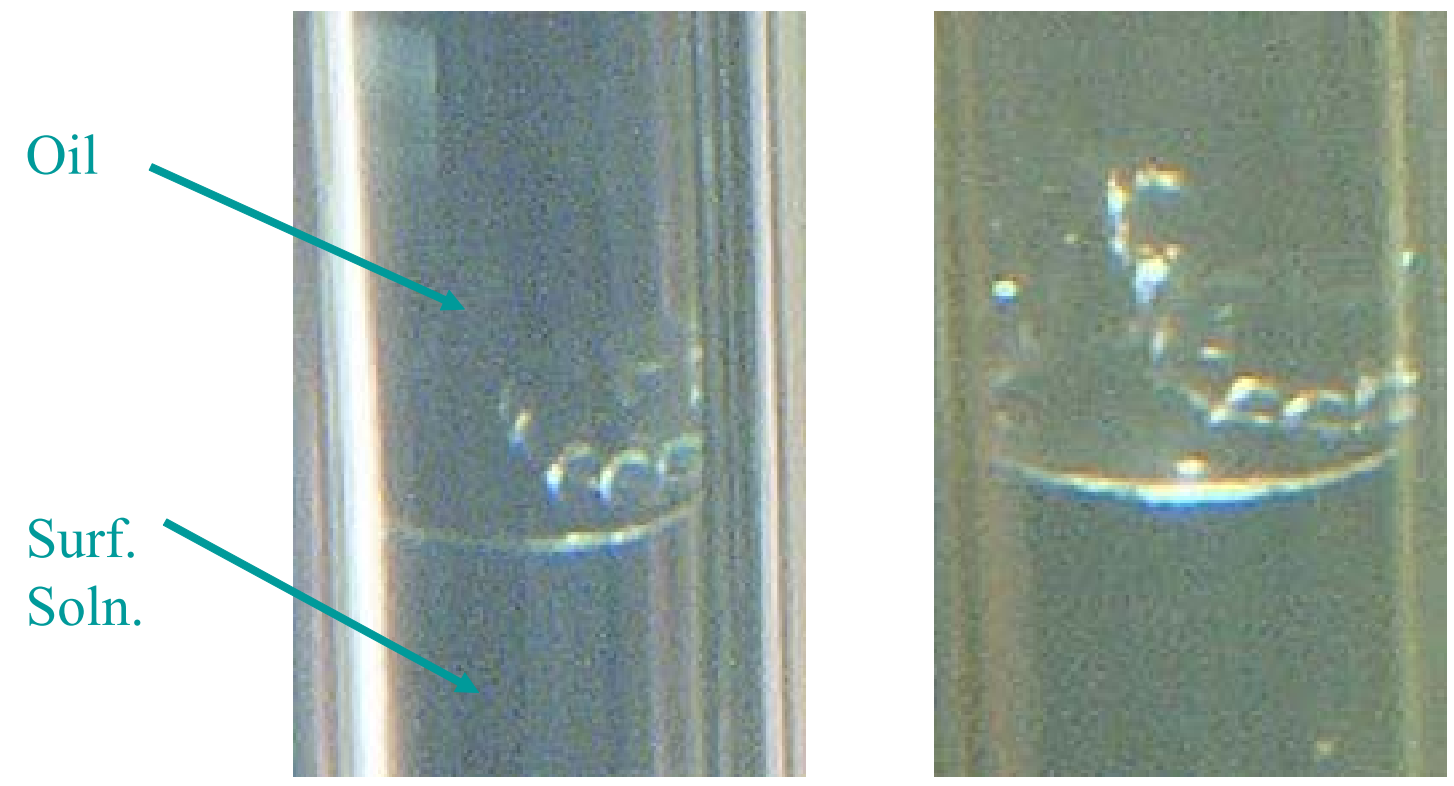

Fig. 1-25. $\mathrm{CO}_{2}$ traversed the oil in a string of connected bubbles.
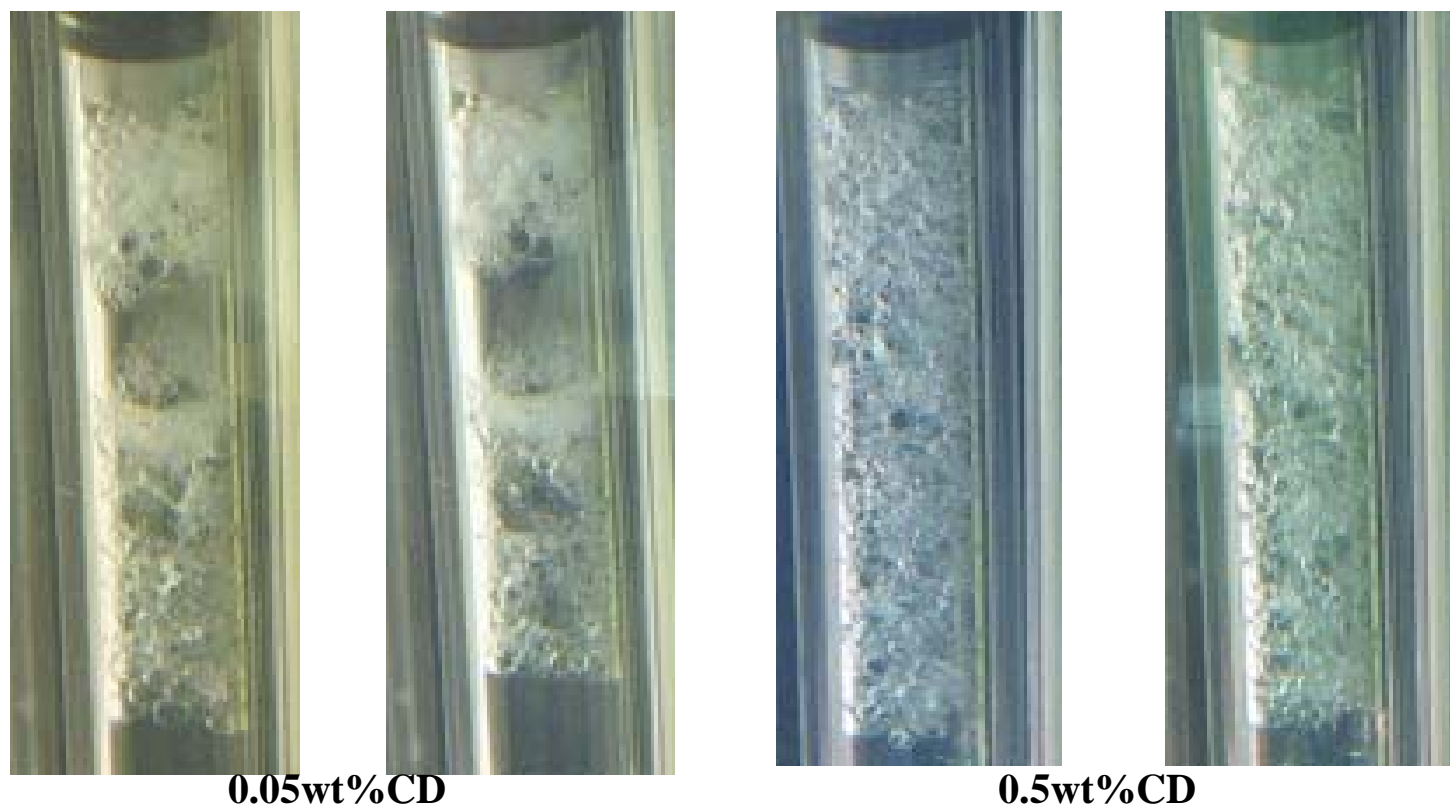

Time since bubble generation ended

0 min

0 min

$90 \mathrm{~min}$

Fig. 1-26. Foam stability at different CD concentrations. 


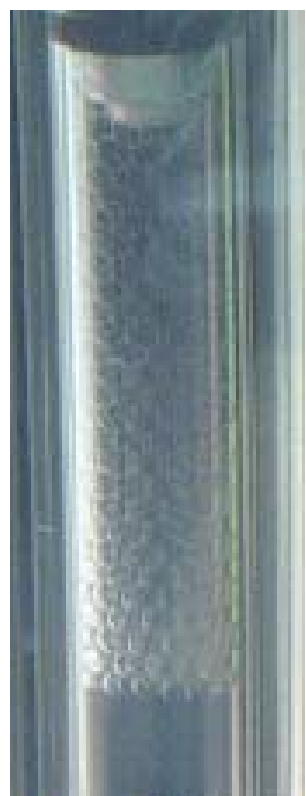

$0.1 \mathrm{wt} \% \mathrm{CD}$

Salinity: $2 \mathrm{wt} \%$

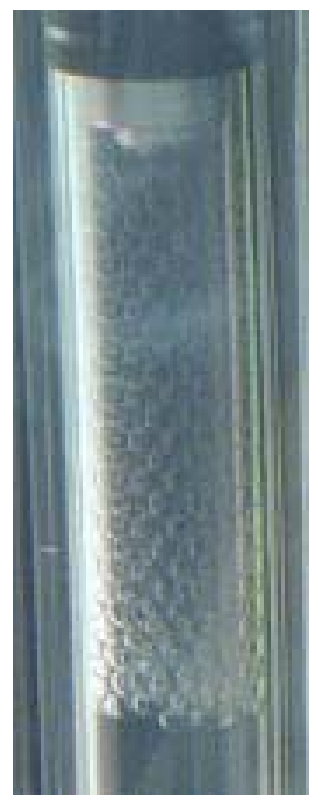

$25 \mathrm{wt} \%$
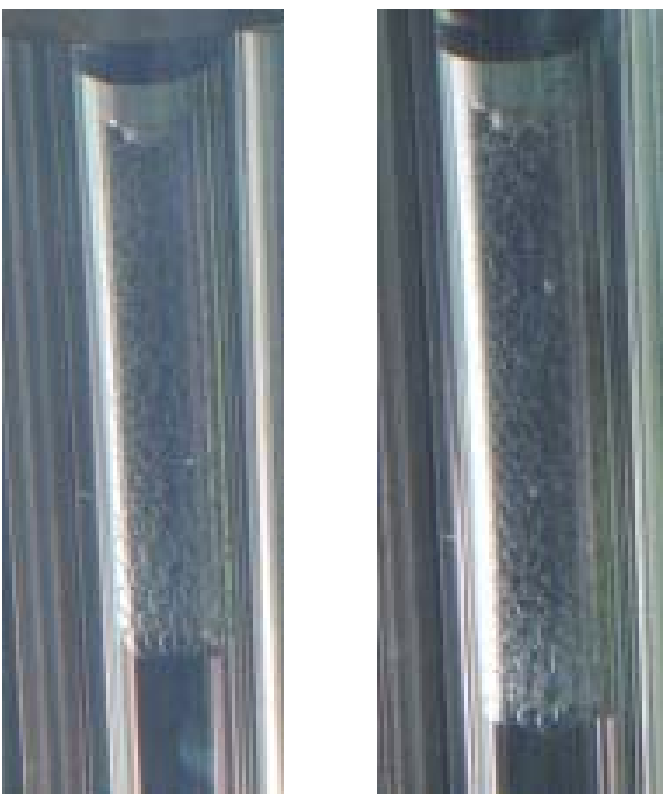

0.05 wt \% CD

$2 \mathrm{wt} \% \quad 25 \mathrm{wt} \%$

Fig.1-27. Salinity versus $\mathrm{CO}_{2}$ foam stability.

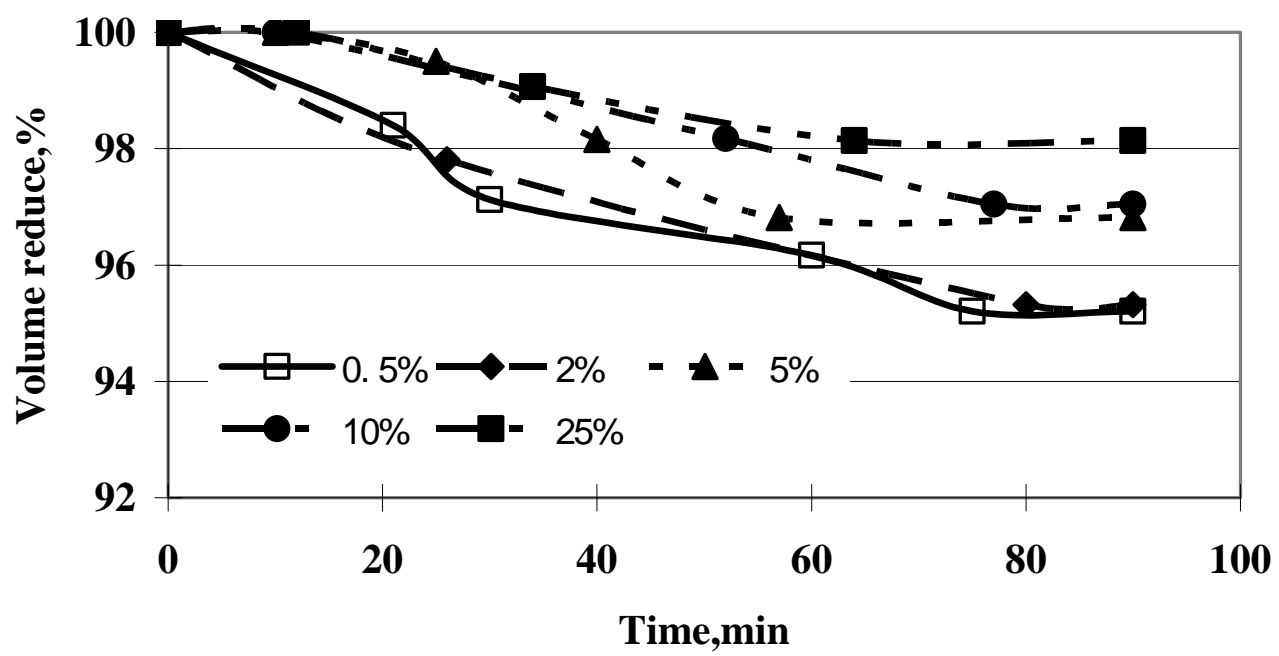

Fig. 1-28. Gravity drainage at different salinities. 


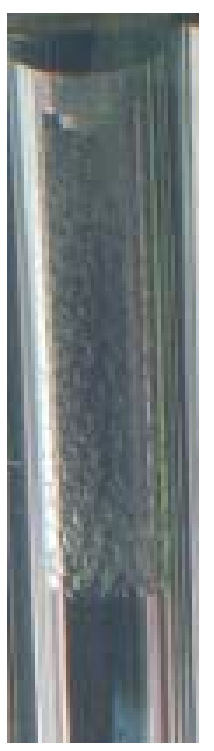

$25^{\circ} \mathrm{C}$

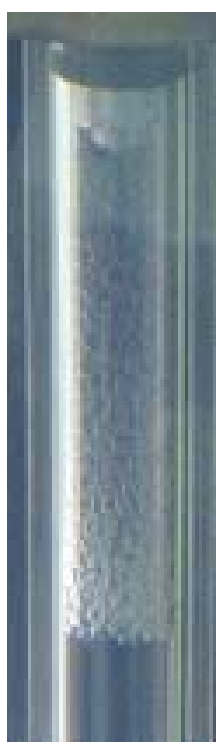

$40^{\circ} \mathrm{C}$
$0.025 w t \%$ CD

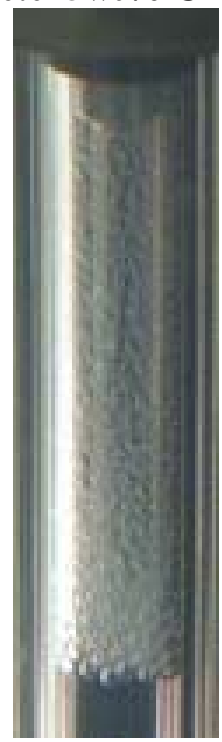

$60^{\circ} \mathrm{C}$

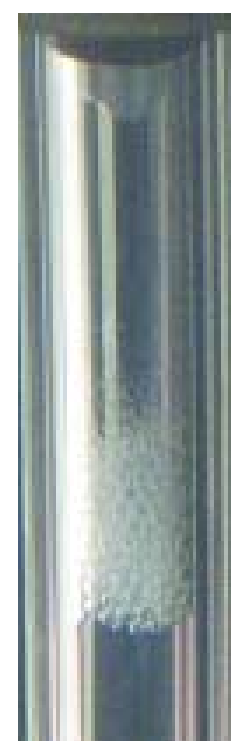

$75^{\circ} \mathrm{C}$ unstable foams

0 minutes

0.1wt \% CD

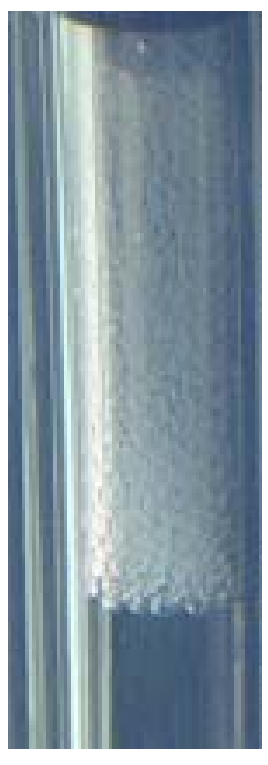

$75^{\circ} \mathrm{C}$

$75^{\circ} \mathrm{C}$

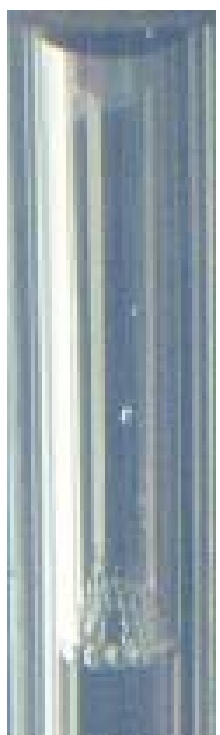

$75^{\circ} \mathrm{C}$

5 minutes

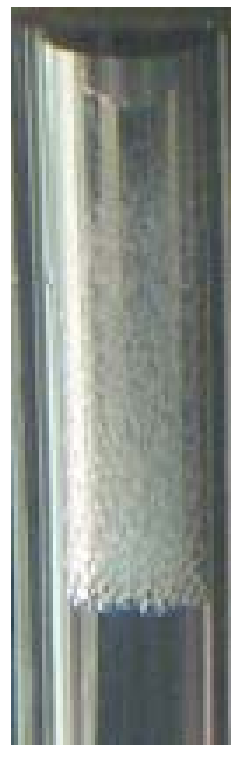

90 min.

17 hrs.

Fig. 1-29. Temperature effect on $\mathrm{CO}_{2}$ foam stability. 

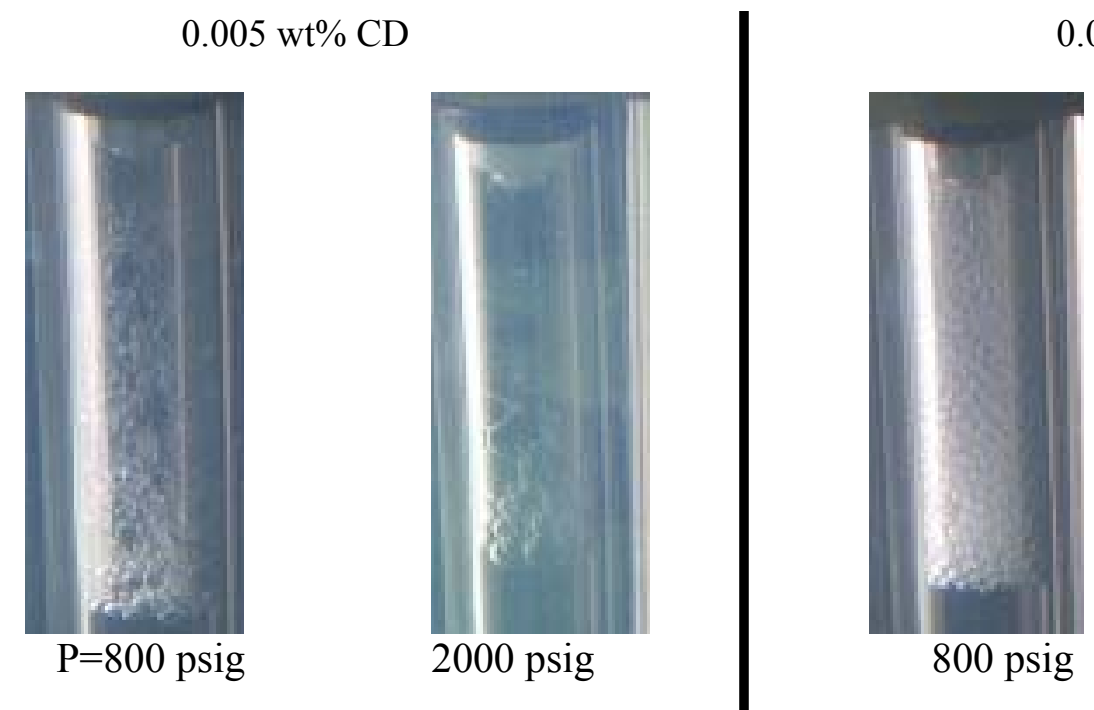

$0.025 w t \% C D$

Fig. 1-30 Pressure effect on $\mathrm{CO}_{2}$ foam stability.

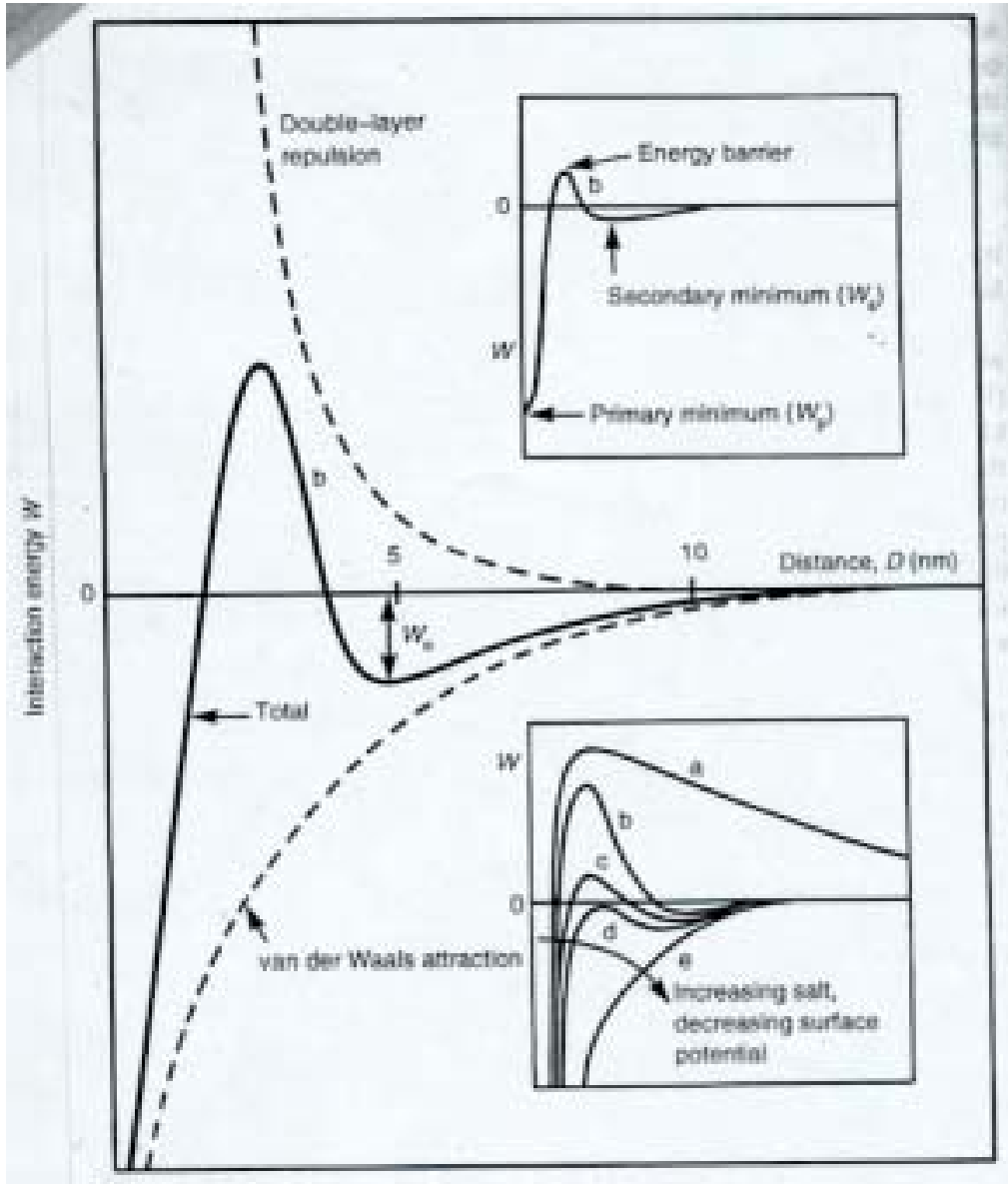

Fig. 1-31. DLVO theory. ${ }^{31}$ 

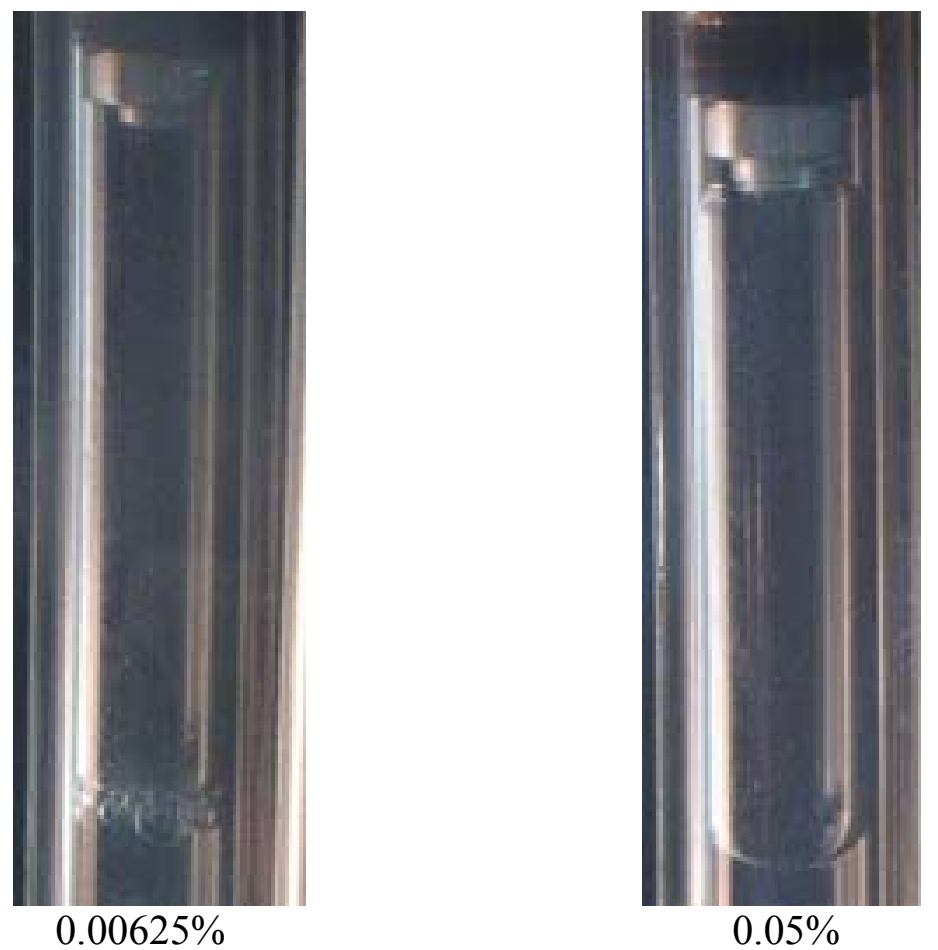

Fig. 1-32. Effect of HPAM concentration in $2 \%$ brine solution after bubble generation.
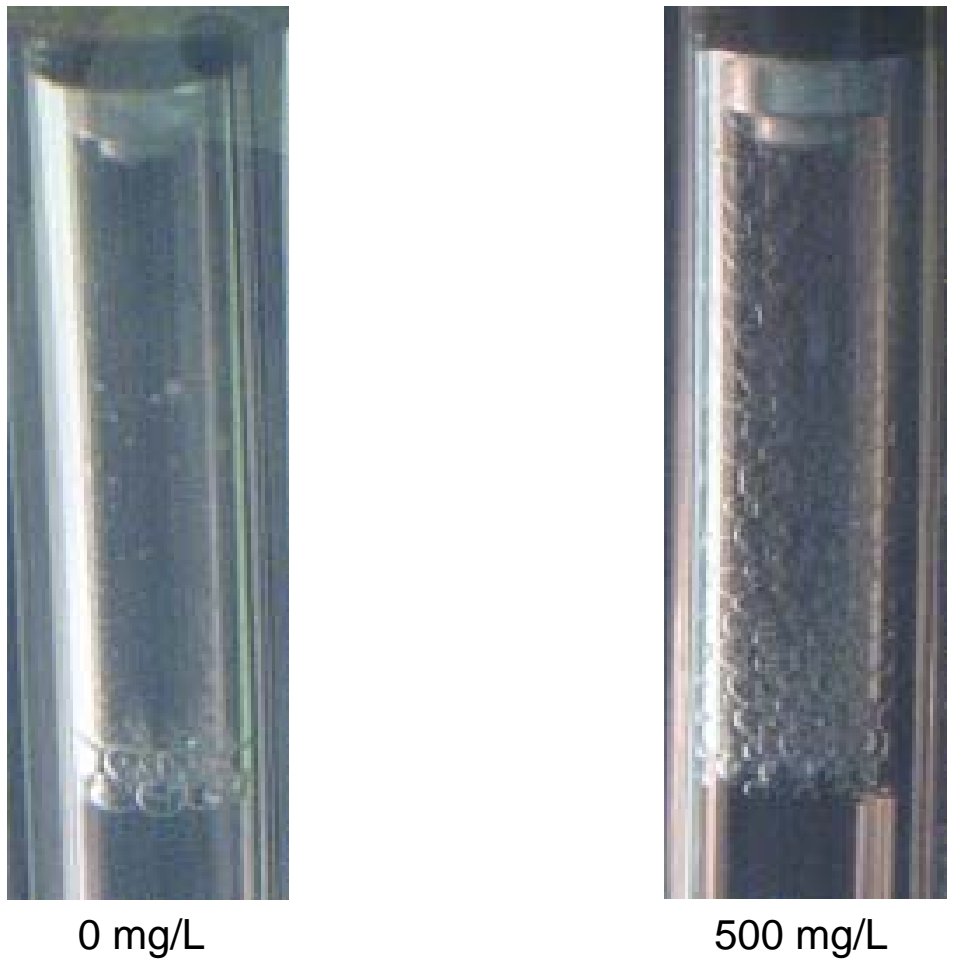

Fig. 1-33. Effect of HPAM concentration on $\mathrm{CO}_{2}$ coalescing in a solution of $0.005 \% \mathrm{CD} 1045+2 \%$ brine. 


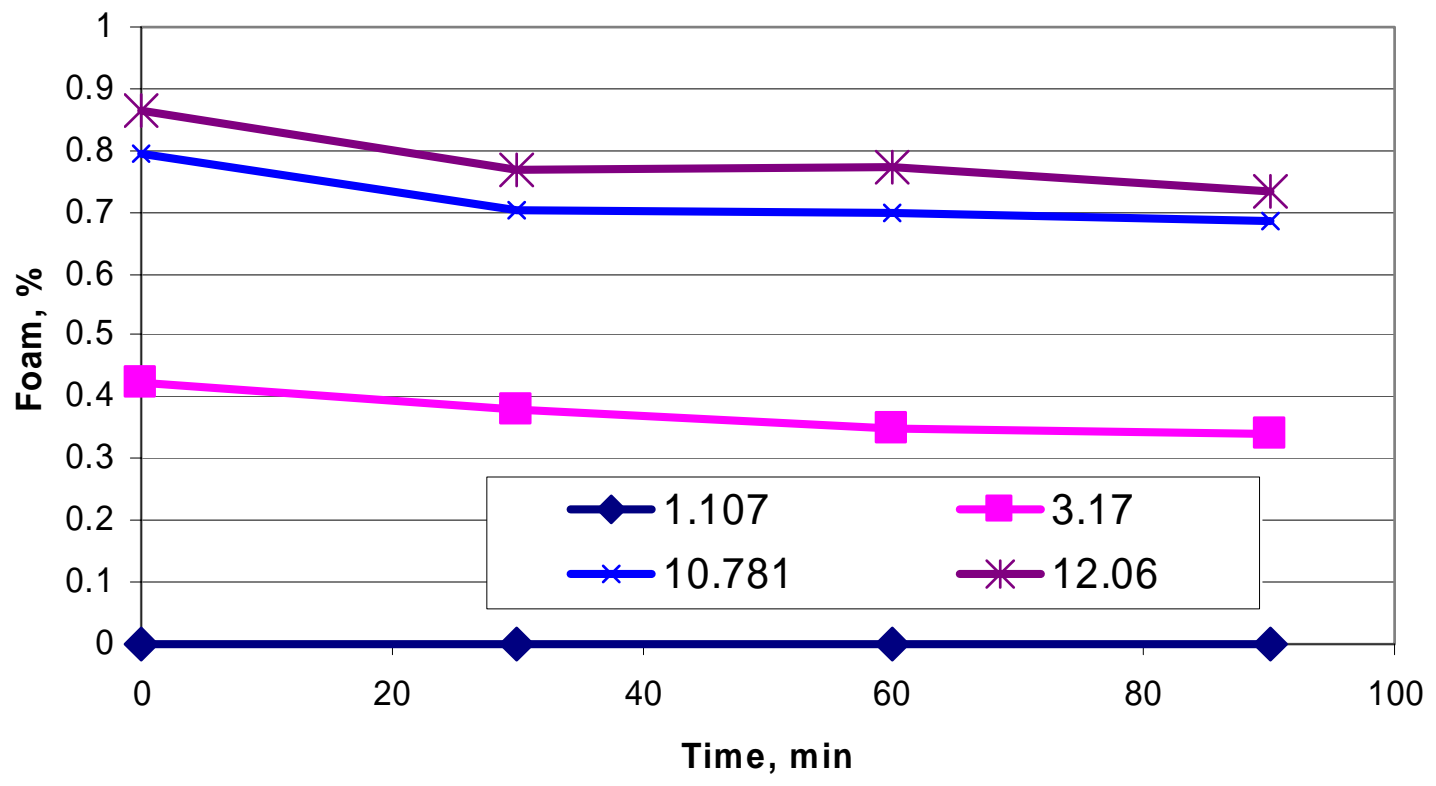

Fig. 1-34. Foam stability at $0.005 w t \%$ CD 1045 in $2 \%$ brine.

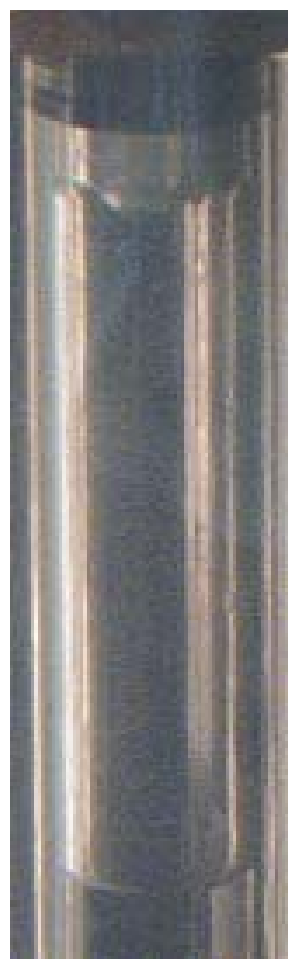

$\mathrm{pH}$ :

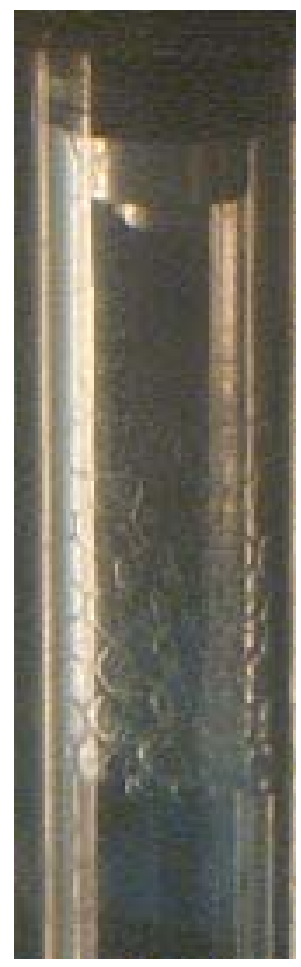

3.17

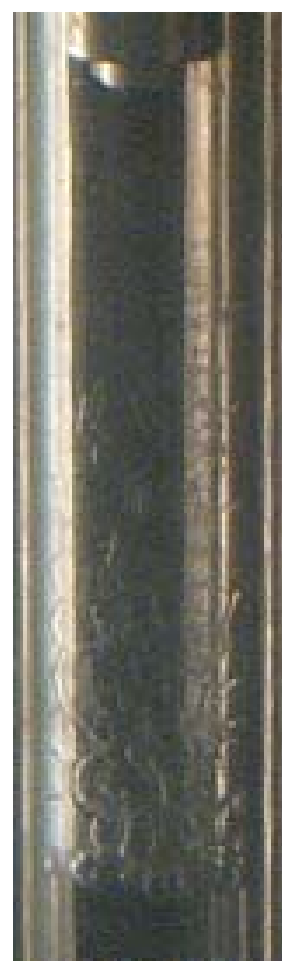

10.871

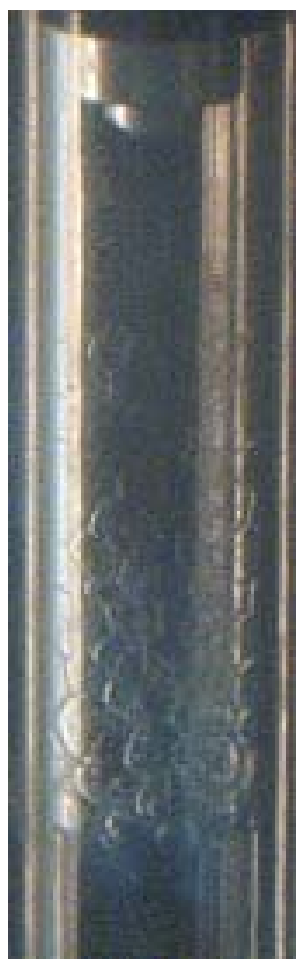

12.06

Fig. $1-35$. Foam images at $0.005 \%$ CD 1045 in $2 \%$ brine. 


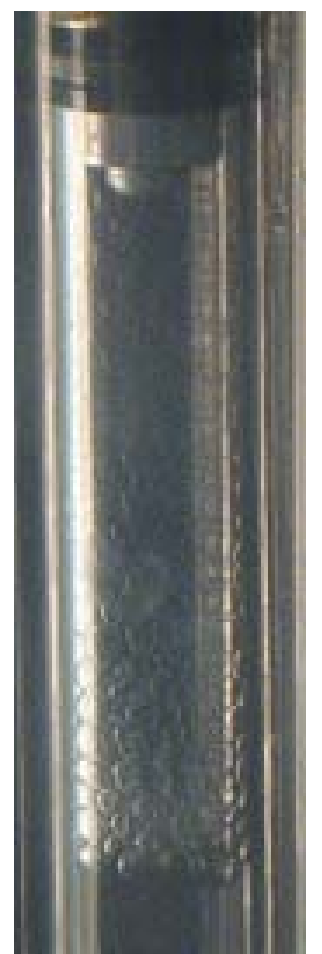

1.537

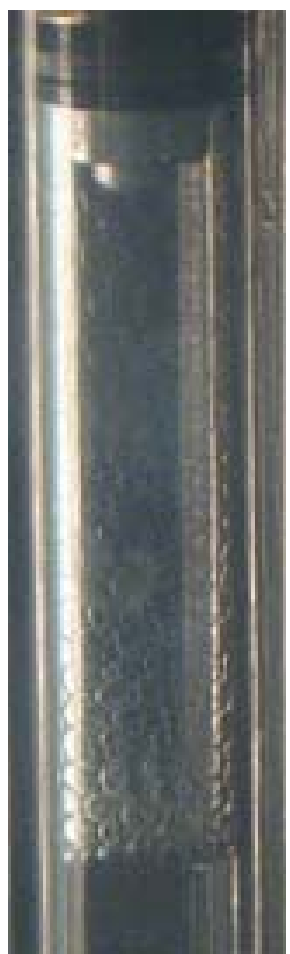

3.033

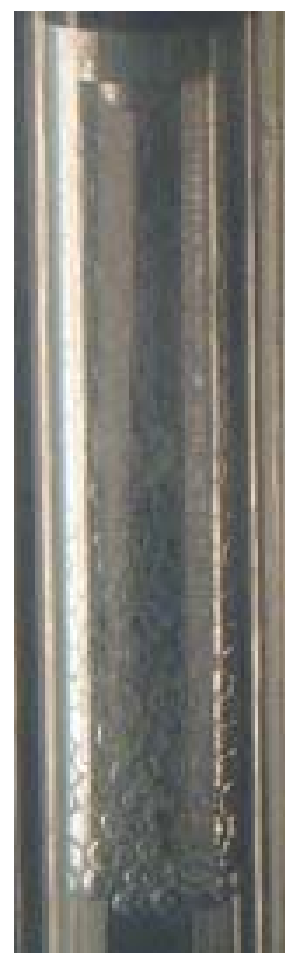

11.14

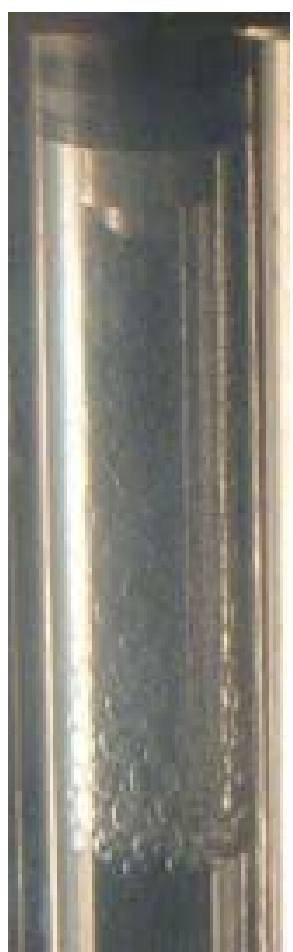

12.724

Fig. 1-36. Effect of indicated $\mathrm{pH}$ on $\mathrm{CO}_{2}$ foam stability at $0.05 \% \mathrm{CD}$ in $2 \%$ brine.

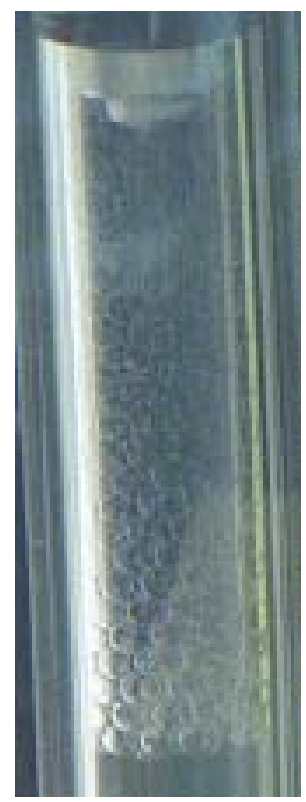

$0.05 \% \mathrm{CD}$

Before

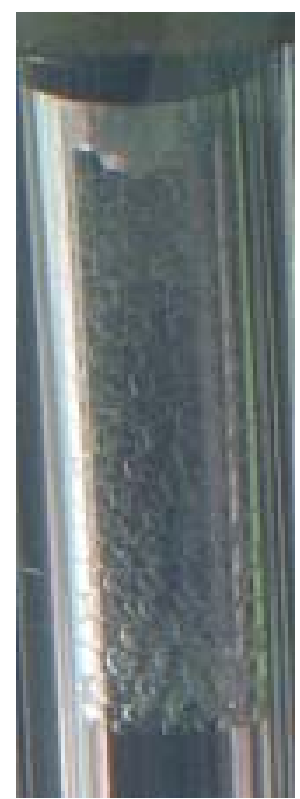

$0.01 \% \mathrm{CD}$

Before
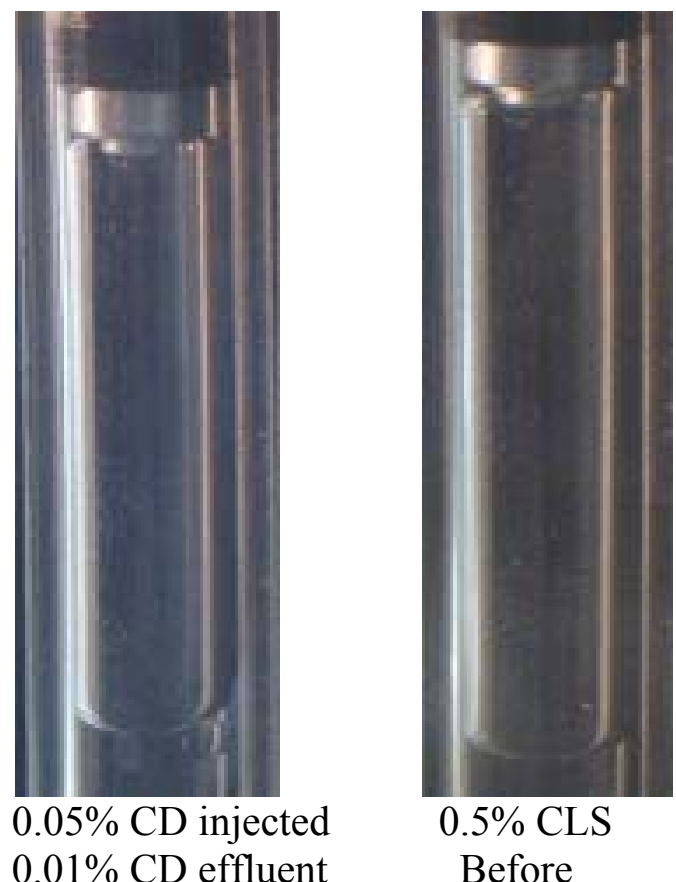

Before

Fig. 1-37. Stability before and after adsorption onto the limestone at $90 \mathrm{~min}$. 


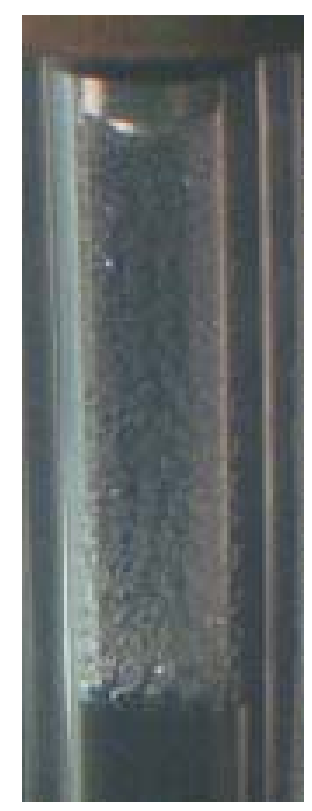

Before adsorption $0.5 \% \mathrm{CLS}+0.05 \% \mathrm{CD}$

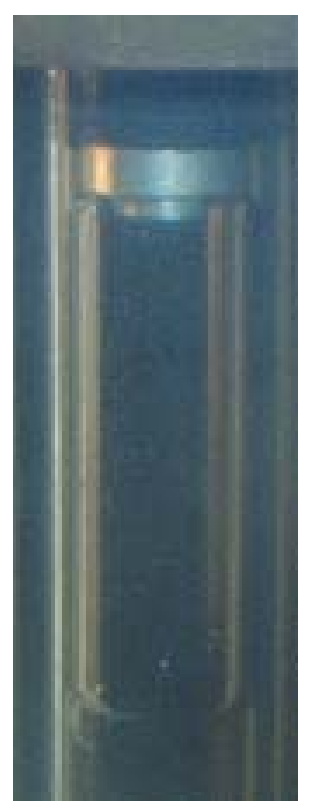

Before adsorption

Preflush $0.5 \%$ CLS then 0.05 CD

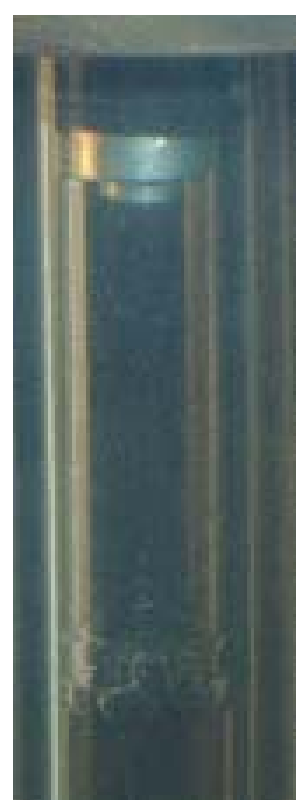

Before adsorption $0.5 \% \mathrm{CLS}+0.05 \% \mathrm{CD}$

Fig. 1-38. Stability before and after adsorption in the limestone for hybrid surfactant systems.

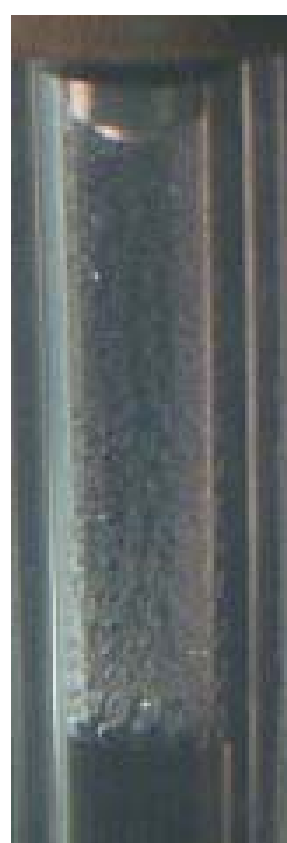

Mixture

$0.5 \% \mathrm{CLS}+0.05 \% \mathrm{CD}$

Before

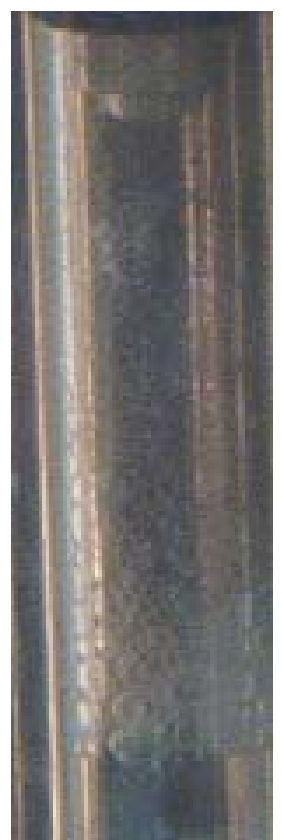

Mixture

$0.43 \%$ CLS $+0.02 \%$ CD

Before

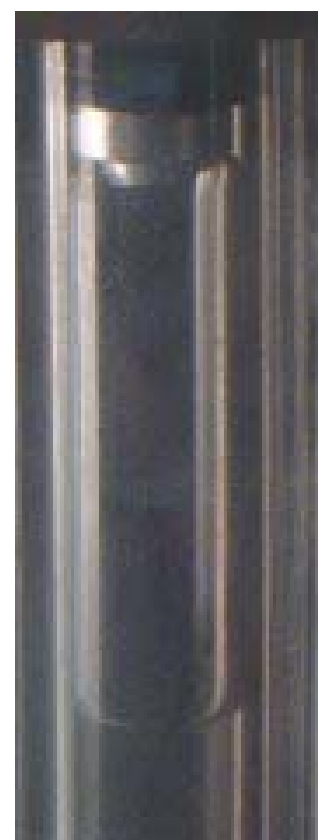

Preflush

$0.5 \%$ CLS followed by $0.05 \% \mathrm{CD}$

After

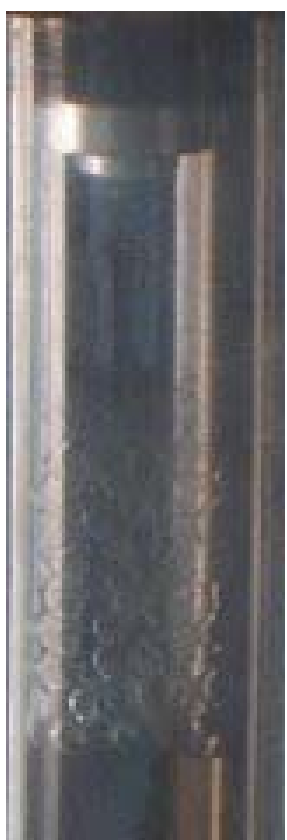

Mixture $0.5 \% \mathrm{CLS}+0.05 \% \mathrm{CD}$

After

Fig. 1-39. Stability before and after adsorption in the Berea sandstone for hybrid surfactant system. 


\title{
CHAPTER 2. CALCIUM LIGNOSULFONATE TRANSPORT THROUGH BEREA SANDSTONE
}

\author{
Abstract: \\ This chapter presents the experiment results of calcium lignosulfonate (CLS) adsorption onto \\ Berea sandstone and five minerals that mainly constitute Berea sandstone, and discusses CLS \\ transport mechanisms through Berea sandstone.
}

The equilibrium time of CLS adsorption onto porous Berea sandstone and its non-porous main component minerals were compared. Results show that the equilibrium time for the former is much longer than that for the latter. Indications are that diffusion is the main mechanism controlling CLS transport through Berea sandstone. Effects of flow interruption or changing postflush rate on effluent concentrations were investigated during desorption. Results show that in a coreflood CLS desorption is a non-equilibrium process under normal reservoir flow rate.

CLS adsorption densities onto five common reservoir minerals were compared. Results show that silica, which constitutes more than $80 \%$ of Berea sandstone and is the bone structure of sandstone, adsorbs little CLS, and that other minerals found in the pores of sandstone contribute most of the adsorption of CLS onto Berea sandstone.

A dual porosity model is suggested to simulate CLS transport through Berea sandstone, even though sandstone is generally regarded as a homogeneous porous medium.

\section{Introduction}

The use of sacrificial agents in either a preflush or the chemical slug can be beneficial for surfactant-based flooding in several ways. Sacrificial agents can alter surfactant loss by preferential adsorbing onto the mineral surface or by reducing exchangeable divalent cations, which can cause surfactant precipitation or loss through phase partitioning. In some cases, these additives may improve the chemical movement through the reservoir by altering fluid mobility.

Sacrificial agents can be inorganic or organic chemicals. Lignosulfonate, a modified waste 
product from the paper industry, has been studied as a sacrificial agent to reduce surfactant adsorption for $\mathrm{CO}_{2}$ foam flooding. The use of lignosulfonate as a sacrificial agent in $\mathrm{CO}_{2}$ foam application was first reported in a patent by Kalfoglou, et al. ${ }^{1}$ They found that lignosulfonate reduced a foaming agent's adsorption on limestone crushed rock samples by 16 to $35 \%$.

The Gas Flooding Processes and Flow Heterogeneities Section (GFPFH) at the New Mexico Petroleum Recovery Research Center (PRRC) has shown that using calcium lignosulfonate (CLS) as a sacrificial agent and a co-surfactant shows synergistic improvement when mixed with the good foaming agents, such as $\mathrm{CD}$ :

- Tsau and Heller established CD adsorption isotherm onto limestone, Baker dolomite, and reservoir dolomite rock samples using distilled water and 4\% synthetic brine at room temperature. ${ }^{2,3}$

- Tsau, Syahputra and Grigg established CD and CLS adsorption isotherms onto Indiana limestone at room temperature and demonstrated that CLS could be used as a sacrificial agent to reduce the adsorption of $\mathrm{CD} .{ }^{4,5,6}$

- Grigg compared the cost reduction by using CLS as a sacrificial agent and cosurfactant according to lab experiment and field test results. ${ }^{7}$

In earlier publications, we reported the adsorption and desorption equilibria of calcium lignosulfonate onto Berea sandstone and five minerals common to reservoirs. ${ }^{8,9}$ The effects of surfactant concentration, temperature, salinity, $\mathrm{pH}$ and injection rate on CLS adsorption and desorption were studied and reported. In this chapter, the kinetics of CLS adsorption onto Berea sandstone and four minerals prevalent in Berea sandstone were studied and compared to understand the transport mechanism of CLS through sandstone.

\section{Experimental}

\section{Materials}

Surfactants. Lignosulfonate used in this study is Lignosite ${ }^{\circledR} 100$ calcium lignosulfonate. All lignosulfonate solutions in this work were prepared in $2 \mathrm{wt} \%$ brine $(1.5 \mathrm{wt} \% \mathrm{NaCl}$ and $0.5 \mathrm{wt} \%$ $\mathrm{CaCl}_{2}$ ). A spectrophotometer was used to determine the concentration of CLS. A $283 \mathrm{~nm}$ wavelength was used in all measurements to analyze the CLS concentration. To calculate CLS 
concentration, a standard calibration curve of CLS in $2 \mathrm{wt} \%$ brine was established (as shown in Fig. 2-1). CLS was diluted to less than $400 \mathrm{mg} / \mathrm{l}$ before its concentration was measured, because concentrations versus absorbance show a straight line only at concentrations below $400 \mathrm{mg} / \mathrm{l}$.

Adsorbents. Five minerals common in oil reservoirs were used as adsorbents: silica, kaolinite, montmorillonite, calcite, and dolomite. All minerals are non-porous. Table 2-1 lists their sources and their chemical compositions.

Silicates are oxides of silicon with traces of other elements constructed of $\mathrm{SiO}_{4}$ tetrahedra that share all four corners with other $\mathrm{SiO}_{4}$ tetrahedra. ${ }^{10}$ Kaolinite is $2 \mathrm{Al}_{2} \mathrm{Si}_{2} \mathrm{O}_{5}(\mathrm{OH})_{4}$ or $2 \mathrm{SiO}_{4} \cdot \mathrm{Al}_{2} \mathrm{O}_{3} \cdot 2 \mathrm{H}_{2} \mathrm{O}$ per unit cell, with no isomorphous substitutions. The montmorillonite is a Wyoming bentonite composed primarily of sodium montmorillonite. It is a hydrous aluminum silicate approximately represented by the formula: $4 \mathrm{SiO}_{2} \cdot \mathrm{Al}_{2} \mathrm{O}_{3} \cdot 2 \mathrm{H}_{2} \mathrm{O}+$ water; but with some of the aluminum cations, $\mathrm{Al}^{3+}$, being displaced by magnesium cations, $\mathrm{Mg}^{2+}$. The name sodium montmorillonite refers to clay minerals in which the loosely held cation is $\mathrm{Na}^{+}$ion. ${ }^{11}$ Calcite and dolomite are carbonate minerals with similar structures. Calcite is formed by alternate layers of calcium ions and carbonate ion groups. Dolomite is composed of alternate layers of calcium ions, magnesium ions, and carbonate ions. Both solids are salt-type minerals; therefore, their solubility in water is higher than oxides and silicates.

Two Berea cores (B02 and B03) were used to determine dynamic adsorption of CLS. Their properties are summarized in Table 2-2.

Tracer: Tracer experiments were performed to characterize Berea sandstone B04 listed in Table 2-2. Sodium iodium (NaI) was used as a tracer. It has a peak at the wavelength of $226 \mathrm{~nm}$. The absorbance has good linear relationship when $\mathrm{NaI}$ concentration is below $40 \mathrm{ppm}$. In the tracer experiments, $200 \mathrm{ppm}$ of NaI solution prepared by $2 \%$ brine were used.

\section{Experimental Methods}

CLS adsorption onto Berea sandstone. Two dynamic methods, circulation and flow-through experiment, were used to study CLS adsorption and desorption onto Berea sandstone. The 
amount of CLS adsorbed is expressed as the unit: mass of CLS adsorbed per weight of rock $(\mathrm{mg} / \mathrm{g})$. Figure 2-2 shows a schematic diagram of the flow-through method apparatus. The source fluid is pumped from a beaker through the pump and into the core holder containing a core. Fluid effluent samples were collected versus time and the concentrations of surfactant were analyzed by spectrophotometer. Figure 2-3 shows a flow chart of the circulation experiment. As shown in this figure, the circulation experimental apparatus consists of:

- A given solution having a known weight in a flask;

- A core of known volume and weight; and

- A metering pump.

A known concentration surfactant solution was circulated through the core to determine adsorption dynamic and adsorption equilibrium.

CLS adsorption onto five minerals: CLS adsorption density onto five minerals was measured by a static experiment method. Figure 2-4 shows the schematic diagram of the static experiment. The objectives of static experiment are to analysis adsorption dynamic and to determine adsorption equilibrium time and adsorption density of CLS onto different minerals. Static adsorption density is obtained by measuring depletion in solute concentration at some time after putting solutions of a known initial concentration and weighed quantities of the dry solids together.

Tracer experiments: The experiment setup and procedures are the same as those of the flow through experiment shown in Fig. 2-2.

\section{Results and Discussion}

\section{CLS adsorption and desorption onto five minerals.}

X-ray diffraction analysis showed that Berea sandstone was composed of 85 90\% silica, 3 6\% feldspar, 1-2\% dolomite, 5-6\% kaolinite, $1 \%$ illite and some other trace components, such as smectite. ${ }^{12,13}$ In our study, five minerals, including silica, kaolinite, dolomite, calcite and montmorillonite (one type of smectite), were selected to determine CLS adsorption dynamic process and to compare CLS adsorption density onto the different components of Berea sandstone. The reason that montmorillonite was selected is that it has a large surface area due to 
its small size $(<2 \mathrm{um})$ even though it is found at relatively low concentrations in Berea sandstone.

Static experiments were performed to study CLS adsorption onto five minerals. In the experiments, a designed volume of solution with a desired CLS concentration was pipetted into a bottle and a weight amount of mineral. The bottle with solution and the mineral were kept in a thermostatic bath to allow the solution to achieve thermal equilibrium. Then the mineral was added into the bottle and shaken vigorously by hand for about a minute. In the experiment to determine adsorption process, the bottle was shaken in the thermostatic shaker bath and samples were taken at designed intervals. For the experiments that determined adsorption density, the bottle was shaken for 24 hours and then left undisturbed for another 48 hours. After pipetting a sample, the sample was centrifuged at $2000 \mathrm{rpm}$ for 20 minutes, and the supernatant solutions were separated by decantation from the vial of the solids after gravity sedimentation. The concentration difference between the stock and the sample was used to evaluate the adsorption density.

CLS adsorption process onto five minerals: Two series of experiment were carried out. For the first series of experiments, the designed volume of solution was $12 \mathrm{~cm}^{3}$ and the adsorbents weight was 4 grams except for 3 grams of montmorillonite, as it swells extensively in solution. A sample was taken every $24 \mathrm{hrs}$ to measure its concentration and a total of five samples were taken from each system of adsorbate and adsorbent. The initial CLS concentration is 5, 000 mg/L. Figure 1-5 shows the adsorption dynamic of CLS onto the five adsorbents. The CLS adsorption densities remained constant after the time the first sample was taken. CLS adsorption onto these four minerals achieves equilibrium in less than $24 \mathrm{hrs}$. Also, the CLS adsorption densities have large differences. The order of decreasing adsorption density is montmorillonite, kaolinite, dolomite, calcite and silica with the adsorption of CLS onto silica being essentially zero.

The second series of experiment was designed to determine how long it takes for adsorption and desorption to reach equilibrium. The designated volume of solution is $100 \mathrm{~cm}^{3}$. Considering the components of Berea sandstone, silica and kaolinite were selected, with weights of $100 \mathrm{~g}$ and 10 
$\mathrm{g}$ respectively. For the desorption process, a total of $50 \mathrm{~cm}^{3} \mathrm{CLS}$ solution (including the volume of samples taken out) was taken out from the equilibrium adsorption system and $50 \mathrm{~cm}^{3}$ of $2 \%$ brine was then added to dilute the left CLS solution in the system of adsorbate and adsorbent.

For both the adsorption and desorption process, the time of sampling was $0.5,1,3,6,9,12$, 24,36, 48, 72, 96 and $120 \mathrm{hrs}$ after mixing the solution and adsorbents together. The initial CLS concentration was $5,000 \mathrm{mg} / \mathrm{L}$. Figure 2-6 shows the adsorption and desorption profiles. Similar to the first adsorption experiment, the CLS adsorption onto silica is essentially zero; the adsorption process cannot be discerned due to the scatter of the data. For the kaolinite, the equilibrium time is very much shorter, less than $0.5 \mathrm{hrs}$, for both the adsorption and desorption processes. Here it should be noted that all minerals selected are non-porous, so adsorption only takes place on the surface of these minerals. It indicates that the physical chemistry process will only take a short time to reach equilibrium for the system of CLS and non-porous media. For the system of CLS and kaolinite, the average adsorption rate is more than $10 \mathrm{mg} / \mathrm{g} / \mathrm{hr}$.

Adsorption isotherm of CLS onto five minerals: Adsorption isotherms of CLS onto five minerals were determined separately. Figure 2-6 shows these results. The adsorption density increases with concentration for all systems. CLS adsorption density onto silica is essentially zero. The order of CLS adsorption at equal concentrations onto these five minerals is: montmorillonite > kaolinite > dolomite >calcite> silica. The differences depend on the mineral surface properties and their surface area. For many surfactants, the adsorption isotherm will plateau at surfactant concentrations greater than its CMC, but no plateau was found for CLS adsorption onto all tested minerals to CLS concentrations greater than $10,000 \mathrm{mg} / \mathrm{l}$. This is believed to be because lignosulfonates lack of amphiphilicity and cannot form micelles. ${ }^{14}$

\section{CLS adsorption and desorption onto Berea sandstone}

CLS adsorption process onto Berea sandstone: Circulation experiments were carried out to determine CLS adsorption dynamics onto Berea sandstone. Earlier experimental results demonstrated that injection rate has little effect on adsorption density of CLS onto Berea sandstone. ${ }^{8,9}$ The injection rate was $0.5 \mathrm{~cm}^{3} / \mathrm{min}$. A total of $100 \mathrm{~cm}^{3}$ (including the brine volume in the core and dead volume of the pump system) of 5,000 mg/l CLS solution was circulated. 
The $5,000 \mathrm{mg} / \mathrm{l}$ is the average concentration of total circulation solution, not the initial concentration in the mixing bottle.

Figures 2-8 and 2-9 show the trends of CLS adsorption density with circulation time. The difference between the two figures is their scale type. Figure 2-8 uses a normal scale (linearlinear) and Fig. 2-9 is a semi-log plot (linear-log). It can be seen from both figures that CLS adsorption onto Berea is a long process, taking more than $72 \mathrm{hrs}$ to reach equilibrium. The adsorption curve can be divided into three regions, represented by three different lines, as shown in both Figs. 2-8 and 2-9. The majority of adsorption occurs in the first region, which is about $80 \%$ of total adsorption density. This stage lasted about nine hours. In this region, the solution in the mixing bottle was diluted by brine in the core and dead volume of the pump system, and adsorption occurs in the pore surface that CLS solution can pass through. As mentioned above, silica adsorbs very little CLS, and thus it can be deduced that some clays and/or carbonate cements exit at the surface of the silica. The second region, shown by another line, occupied about $20 \%$ of total adsorption density. Comparing CLS adsorption process onto non-porous media infers that CLS adsorption was controlled by diffusion. The second process continued for more than 60 hrs. The third region showed a constant adsorption density, which indicates that the final adsorption equilibrium was obtained.

The semi-log plot shown in Fig. 2-9 is often used to analysis adsorption mechanisms. ${ }^{3}$ The linear relationships of the log value of CLS adsorption density and time show different mechanisms controlling CLS adsorption and transportation through Berea sandstone.

When CLS adsorption processes onto five minerals and Berea sandstone are compared, the CLS adsorption rates shows differences between them. There are two differences between the former static experiments and the dynamic experiment. The first difference is that the former are static and the later is dynamic. The second difference is that the adsorbents of the formers are nonporous and the later are porous. The first difference results in adsorption time of the first region for dynamic experiment flow through in a porous media having a longer equilibrium time than the static non-porous experiment. For dynamic experiments, mechanic dispersion is one important mechanism controlling solute transport through porous media. ${ }^{15}$ Dispersion is mixing 
that occurs as a consequence of local variations in velocity around some mean velocity of flow. There are a number of reasons that cause mechanical dispersion for the core experiments. The schematics in Fig. 2-10 demonstrates the three possible causes. ${ }^{15}$ The first is that some pores in the sandstone are larger than others, which allows the CLS flowing through these pores to move faster, as shown in Fig. 2-10(a). The second is that some of the CLS particles will travel along longer flow paths in the porous media than the other particles to go the same linear distance (path length), shown in Fig. 2-10 (b). The third reason is that the fluid will move faster in the center of the pores than along the edges due to viscous friction force effect, wettability variations, adsorption, etc, shown in Fig. 2-10(c). In a reservoir, velocity variations are caused by larger scale heterogeneities, such as layers, cross-beddings, faces changes, and stratifications, so it will take a longer time to reach adsorption equilibrium. In summary, the CLS transport mechanisms in the first region of adsorption process curve is controlled by advective and mechanic dispersion.

For the second region of adsorption performance curve, the CLS adsorption mechanism is regarded as diffusion-controlled adsorption. This can be explained by the pore structure of sandstone. Although Berea sandstone is generally considered to be a homogenous porous medium, its pore-scale structure is not homogenous. One reason is that clays and/or cements exist in the porous media. Figure 2-11 show the microstructure of Berea sandstone and illustrates three types of clay distribution in the rock. ${ }^{16}$ The first type is dispersed shale residing in the pore space. It by means of the dispersed shale coating at the surface of silica that we can explain why so much CLS was adsorbed onto the Berea sandstone at the first region of CLS adsorption process. The second type is laminated shale which alternate layers of shale and sand. This shale has its own porosity constructed by itself. The third type is structural shale, which is individual clay grains, and this type of shale can construct pores by itself or together with silica. The size of pore constructed by the second and the third kind of shales or by both silica and shale is much smaller than that constructed by pure silica. At the condition of normal displacing force, the pores constructed by shale are the main part of dead pores in sandstone. In our experiments, brine trapped in dead pores is static and disconnected with the main flow; the only way that CLS can be moved into the dead-end pore is through mass transfer, due to the difference between CLS concentration in the micro-pore and in the macro-pore, which is mainly because of 
molecular diffusion.

Postflush Rate and Interruption Effect on the Desorption Process. After adsorption equilibrium was established, $2 \%$ brine was injected to displace the CLS solution in the core. A desorption experiment was conducted in core B02 to study the postflush rate effect on desorption. The injection rate changed from $4 \mathrm{~cm}^{3} / \mathrm{hr}$ to $200 \mathrm{~cm}^{3} / \mathrm{hr}$, corresponding to Darcy flow rate from $0.28 \mathrm{ft} / \mathrm{d}$ to $11.03 \mathrm{ft} / \mathrm{d}$. Figure $2-12$ shows that the effluent lignosulfonate concentrations are influenced by the brine flow rate. When flow rates decreased, the effluent sample concentration increased. It showed that desorption is a non-equilibrium process under normal reservoir flow condition in laboratory core. Zhang et al. found the same trend when they studied the mechanism of scale inhibitor adsorption on sandstone. ${ }^{17}$ They attributed the observed results to the difference of fluid transit time and the equilibrium time of the chemical/rock system. The fluid transit time was shorter than the kinetics of desorption of the chemical/rock system, and thus did not have the opportunity to reach equilibrium.

To further study CLS desorption versus the extent of non-equilibrium, a flow interruption experiment was performed. In this test flow was stopped for a time, allowing more time for desorption, and then resumed. Figure 2-13 presents an interruption test results. During running experiments, flow was stopped at 7 pore volumes (PV), 14.7 PV and $21 \mathrm{PV}$ for 12 hours each time. The figure shows that immediately after an interruption the effluent solution concentration increased. Brusseau et al. ${ }^{18}$ found the same trend in bi-porous media; they attributed the physical non-equilibrium to diffusion time (diffusive mass transfer between mobile and immobile zones).

In summary, changing post-flush rate and interruption experiments show that under our tested conditions, CLS equilibrium is not achieved during flow conditions. Zhang et al. and Brusseau et al. indicated different explanations for the slow equilibrium time. Zhang suggested it is chemical or kinetics of desorption at the rock/chemical interface, while Brusseau et al. suggested that in their tests, diffusion time or physical non-equilibrium was the limiting process. Reviewing the desorption tests of CLS from non-porous and the possible pore structures of Berea sandstone, the explanation of Brusseau more closely fits our systems. Effluent concentration that increases after rate is decreased or flow is interrupted indicates that the CLS concentration in the micropores 
(immobile zones) is higher than that in macropores formed by silica (mobile zones). The concentration difference caused CLS to diffuse from micropores to macropores (main path of fluid).

Tracer flow through Berea sandstone: To further check the above explanation about CLS transport mechanisms through Berea sandstone, tracer was injected into Berea sandstone to characterize the core. Iodide anion (200 ppm NaI solution) was used as a tracer in the experiments because it is non-reactive with Berea sandstone. Three breakthrough experiments were carried out in Berea sandstone B04 described in Table 2-2, with about 3 PV of tracer injection followed by the injection of $2 \%$ brine each. The schedule of each experiment is shown in Table 2-3. The injection rate was $40 \mathrm{cc} / \mathrm{hr}$ for the first two experiments and $8 \mathrm{cc} / \mathrm{hr}$ for the third one. The difference between the first two experiments was their different interruption time. Figure 2-14 shows the results of the three experiments. For all the three experiments, a rapid tracer concentration increase was followed by a slow increase during tracer injection, while a rapid initial tracer decline was followed by a very slow decrease over relatively long periods of time (an extended tail) during brine injection. Because NaI does not adsorb onto Berea sandstone, the rapid increase or decrease is attributed to advection/dispersion during flow, while the slow increase and the extending tail is a diffusion-limited process. ${ }^{19}$

Figure 2-15 compares the three curves during the nonsorbing tracer injection. The two curves for the first two experiments almost overlay. It should be noted that the two experiments have the same experimental conditions and the only difference between them is their experiment sequence. The overlaying result indicates the porous structure does not change after the first experiment and the core can be used repeatedly for the following experiments. Comparing the first two experiments with the third one, it can be seen that effluent concentrations for the third experiment are lower than those for the other two experiments after tracer breakthrough from core because the flow rate for the third experiment is much lower than the first two and it has a much longer time to diffuse into micropores in Berea sandstone. It also indicated that the tracer flow is under nonequilibrium state when the flow rate is $40 \mathrm{~cm}^{3} / \mathrm{hr}$.

Figures 2-16 through 2-18 show the interruption effect on the tracer effluent concentration for 
experiment No. 02 and No. 03. Brusseau et al. demonstrated that physical non-equilibrium (i.e. diffusive mass transfer between mobile and immobile regions) is present in the core if the breakthrough curve of nonsorbing solution exhibits concentration increase response after interruption during brine injection. ${ }^{18}$ Figure 2-16 has a concentration rebound for the second stop (11.2 PV) but only a slope change, with no obvious effluent concentration rebound for the first stop (5.18 PV). Upon comparing results with and without interruption (shown in Fig. 2-17), it can be seen that interruption made the effluent concentration (No. 2) higher than no interruption (No. 1). That no obvious rebound was found in the first stop is because advection, dispersion and diffusion occur simultaneously and diffusion is not predominant. Thus, the interpretation of the interruption is important when an interruption experiment is performed. Figure 2-18 shows effluent concentration increases for both stops. All interruption experiments show that mobile and immobile regions exist in the Berea core at the tested rate.

Reviewing the above experiment results, it is inferred that CLS solution through Berea core could be simulated using a dual porosity model. One reason is that CLS does not adsorb onto silica, the bulk bone of Berea sandstone, while the clay that forms micropores contributes all the adsorption of CLS. Another reason is that diffusion is one of the main mechanisms controlling the CLS adsorption. A dual porosity model will better reflect the pore structure of Berea sandstone and CLS transport mechanism through Berea sandstone.

\section{Conclusions}

1. The equilibrium time of CLS adsorption on porous Berea sandstone is much longer than its non-porous main component minerals.

2. Diffusion, not kinetics, is the principle mechanism controlling CLS transport through Berea sandstone.

3. CLS desorption onto Berea sandstone is a non-equilibrium process under normal reservoir flow rate.

4. The adsorption of CLS onto silica, the primary component of Berea sandstone, is essentially zero, and other minerals (minor components) contribute essentially all the adsorption of CLS on Berea sandstone. 
5. A dual porosity model is suggested to simulate CLS transport through Berea sandstone, though sandstone is generally regarded as a homogeneous porous medium.

\section{References}

1. Kalfoglou, G., Prieditis, J. and Paulette, G.S.: "Sacrificial Agents for Carbon Dioxide Foaming Agents," Canadian Patent 2,185,499.

2. Tsau J.S. and Heller J.P.: "Evaluation of Surfactant for $\mathrm{CO}_{2}$-Foam Mobility Control," paper SPE 24013 presented at the 1992 SPE Permian Basin Oil and Gas Recovery Conference, Midland, March 18-20.

3. Tsau, J.S. et al.: " $\mathrm{CO}_{2}$ Foam Field Verification Pilot Test at EVGSAU: Phase IIIA: Surfactant Performance Characterization and Quality Assurance," paper SPE 27785 presented the SPE/DOE Ninth Symposium on Improve Oil Recovery, Tulsa, April 17-21, 1994.

4. Tsau, J-S., Syahputra, A. E, Yaghoobi, H., and Grigg, B.R.: "Use of Sacrificial Agents in $\mathrm{CO}_{2}$ Foam Flooding Application," paper SPE 56609 presented at the 1999 SPE Annual Technical Conference and Exhibition, Houston, Oct. 3-6.

5. Syahputra, A.E., Tsau, J-S., and Grigg, R.B.: "Laboratory Evaluation of Using Lignosulfonate and Surfactant Mixture in $\mathrm{CO}_{2}$ Flooding," paper SPE 59368 presented at the 2000 SPE/DOE Improved Oil Recovery Symposium, Tulsa, April 3-5.

6. Tsau, J., Syahputra, A. E. and Grigg, R.: "Economic Evaluation of Surfactant Adsorption in $\mathrm{CO}_{2}$ Foam Application," paper SPE59365 presented at the 2000 SPE/DOE Improved Oil Recovery Symposium, Tulsa, April 3-5.

7. Grigg, R. et al.: “Cost Reduction and Injectivity Improvements for $\mathrm{CO}_{2}$ Foams for Mobility Control," paper SPE 75178 presented at the 2002 SPE/DOE Improved Oil Recovery Symposium, Tulsa, April 13-17.

8. Grigg, R. et al.: "Improving $\mathrm{CO}_{2}$ Efficiency for Recovering Oil in Heterogeneous Reservoirs," Annual technical progress report for the period September 28, 2002 through September 27, 2003, U.S. DOE contract no. DE-FG26-01BC15364 (2003).

9. Grigg, R., Bai, B., "Calcium Lignosulfonate Adsorption and Desorption on Berea Sandstone," Journal of Colloid and Interface Science, (November 2004) 279(1). 
10. Kuehne, D.L. et al.: "Evaluation of Surfactants for $\mathrm{CO}_{2}$ Mobility in Dolomite Reservoirs," paper SPE 24177 presented at the 1992 SPE/DOE Symposium on Enhanced Oil Recovery, Tulsa, April 22-24.

11. Giese, R. F. and Oss, C.J.: Colloid and Surface Properties of Clays and Related Minerals, Marcel Dekker (2002).

12. Churcher L, et al.: "Rock Properties of Berea Sandstone, Baker Dolomite, and Indiana Limestone," paper SPE 21044 presented at the SPE International Symposium on Oilfield Chemistry, Anaheim, February 20-22, 1991.

13. Mannhardt, K. et al.: "Effect of Rock Type and Brine Composition on Adsorption of Two Foam-Forming Surfactants," SPEATS, 1993.

14. Hornof, V.: "Applications of Lignosulfonate in Enhanced Oil Recovery," Cellulose Chemistry and Technology, (1990) 24, 407-415,

15. Fetter, C.W.: Applied Hydrogeology. 2d ed., New York: Macmillan Publishing Company (1988).

16. Djebbar,T. and Erle, D.: Petrophysics-Theory and Practice of Measuring Reservoir Rock and Fluid Transport Properties.\Gulf Professional Publishing 1996

17. Zhang, H. Sorbie, K.S.: "Non-Equilibrium Adsorption and Precipitation of Scale Inhibitors: Corefloods and Mathematical Modelling," paper SPE 64755 presented at the 2000 SPE International Oil and Gas Conference and Exhibition, Beijing, Nov. 7-10.

18. Brusseau, M.L., Rao, R.E. and Davidson, J.M.: "Flow Interruption: a Method for Investigating Sorption Nonequilibrium”. J. Contam. Hydrol, vol 4, 1989:223-240.

19. Grathwohl, P., "Diffusion in Natural Porous Media: Contamination Transport, Sorption/Desorption and Dissolution Kinetics-Chapter 1," Klumer Academic Publisher, 1998

Table 2-1. Source and Main Composition of Five Minerals

\begin{tabular}{|c|c|c|}
\hline Mineral & Source & Chemical composition \\
\hline Montmorillonite & Wyo-Ben Incorp. & $4 \mathrm{SiO}_{2} \cdot \mathrm{Al}_{2} \mathrm{O}_{3} \cdot 2 \mathrm{H}_{2} \mathrm{O}$ \\
\hline Kaolin & Acro Organics & $\mathrm{Al}_{2} \mathrm{Si}_{2} \mathrm{O}_{5}(\mathrm{OH})_{4} \mathrm{OrSiO}_{4} \cdot \mathrm{Al}_{2} \mathrm{O}_{3} \cdot 2 \mathrm{H}_{2} \mathrm{O}$ \\
\hline Silica & Mo-sci Corp. & $\mathrm{SiO}_{2}$ \\
\hline Calcite & J.T. Baker & $\mathrm{CaCO}_{3}$ \\
\hline Dolomite & Naturaceutical Corp. & $\mathrm{CaMg}\left(\mathrm{CO}_{3}\right)_{2}$ \\
\hline
\end{tabular}


Table 2-2. Properties of Berea Core Samples

\begin{tabular}{|c|c|c|c|c|c|}
\hline No & $\begin{array}{c}\text { Permeability } \\
(\mathrm{md})\end{array}$ & $\begin{array}{c}\text { Porosity } \\
(\%)\end{array}$ & $\begin{array}{c}\text { Length } \\
(\mathrm{cm})\end{array}$ & $\begin{array}{c}\text { Diameter } \\
(\mathrm{cm})\end{array}$ & $\begin{array}{c}\text { Weight } \\
(\mathrm{g})\end{array}$ \\
\hline B02 & 320 & 19.20 & 6.10 & 3.75 & 139.92 \\
\hline B03 & 224 & 16.70 & 6.20 & 3.75 & 142.21 \\
\hline B04 & & 13.11 & 7.00 & 3.75 & \\
\hline
\end{tabular}

Table 2-3. Schedule of Tracer Breakthrough Experiments

\begin{tabular}{|l|c|c|c|}
\hline No. & $\begin{array}{c}\text { Injection Rate } \\
\left(\mathrm{cm}^{3} / \mathrm{hr}\right)\end{array}$ & $\begin{array}{c}\text { Tracer Solution Injection } \\
(\mathrm{PV})\end{array}$ & Interruption Point \\
\hline 1 & 40 & 3.12 & 9.14 \\
\hline 2 & 40 & 3.17 & $5.18,11.12$ \\
\hline 3 & 8 & 3.05 & $5.78,11.06$ \\
\hline
\end{tabular}




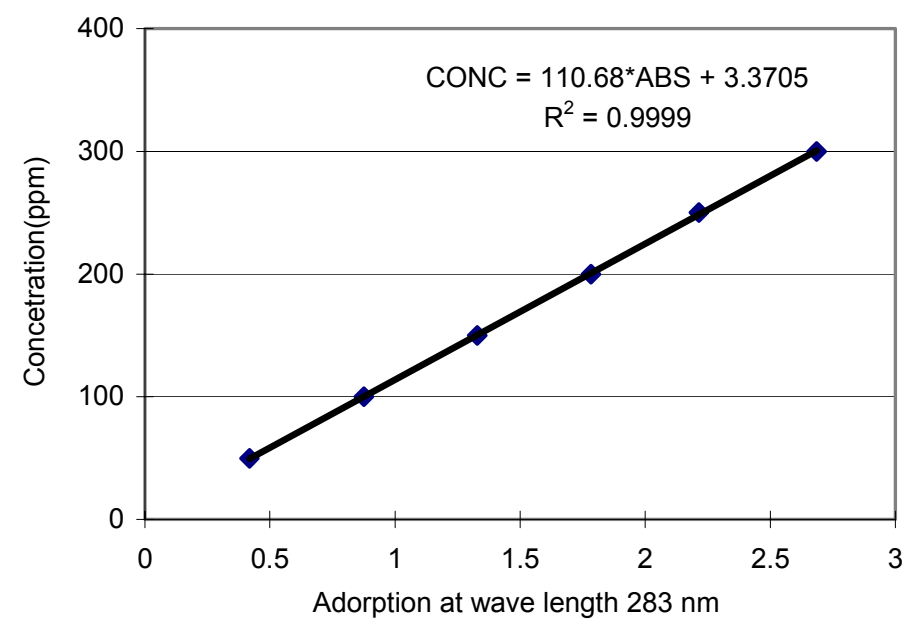

Fig. 2-1. CLS standard curve with wavelength $283 \mathrm{~nm}$.

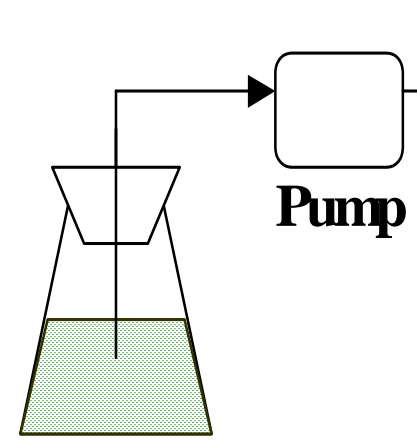

Solution

Fig. 2-2. Schematic diagram of flow-through method. 


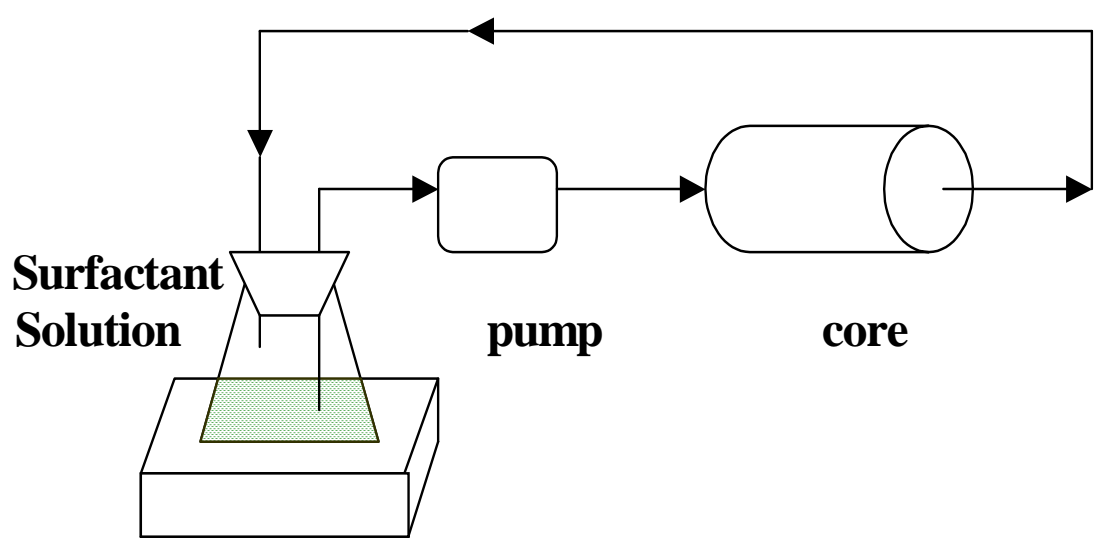

Magnetic stirrer

Fig. 2-3. Schematic diagram of circulation method.

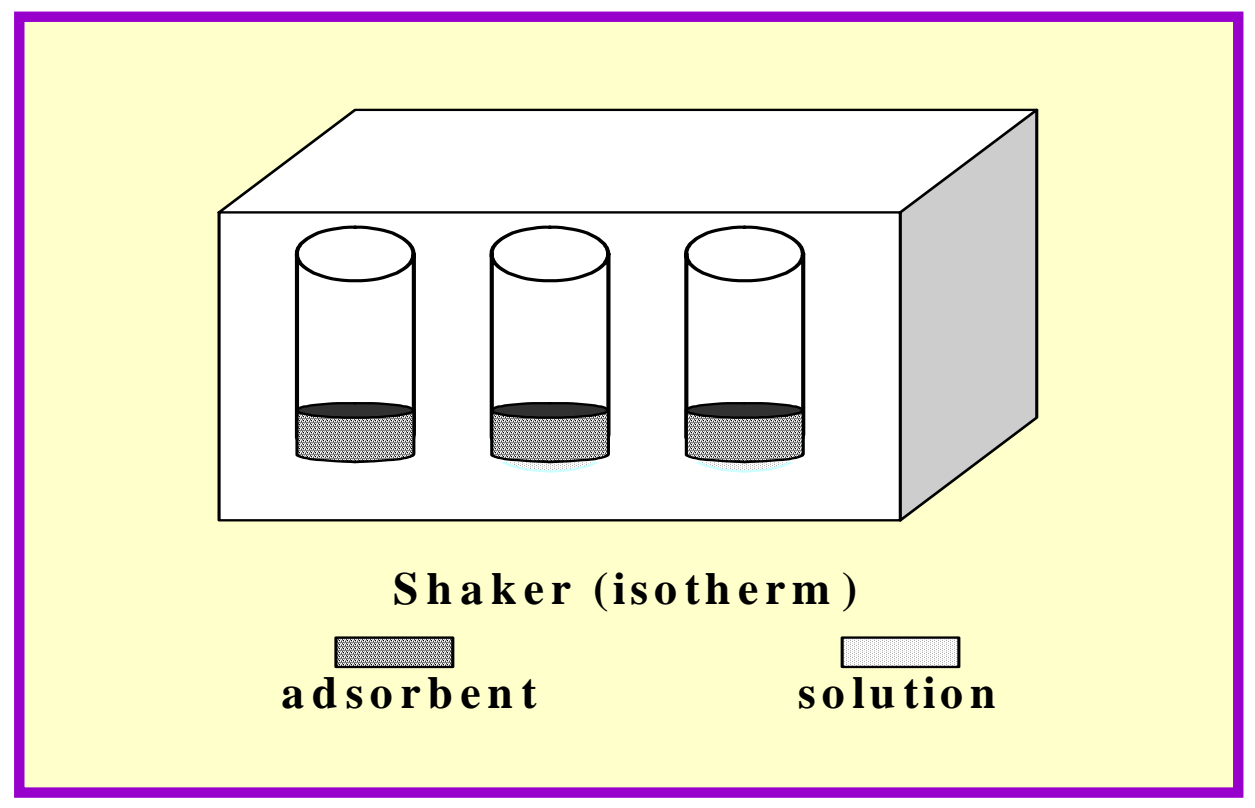

Fig. 2-4. Schematic diagram of static adsorption. 


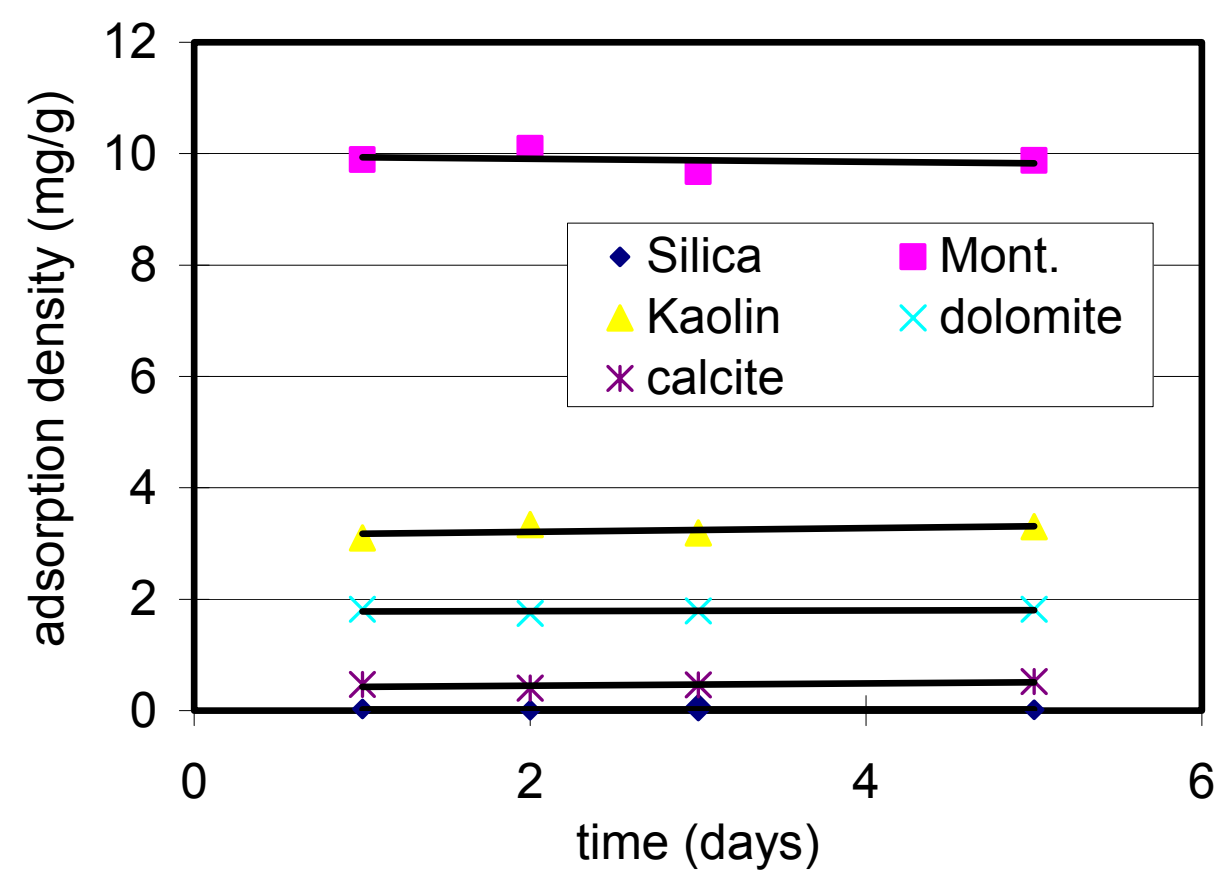

Fig. 2-5. Adsorption dynamic of CLS onto five minerals.

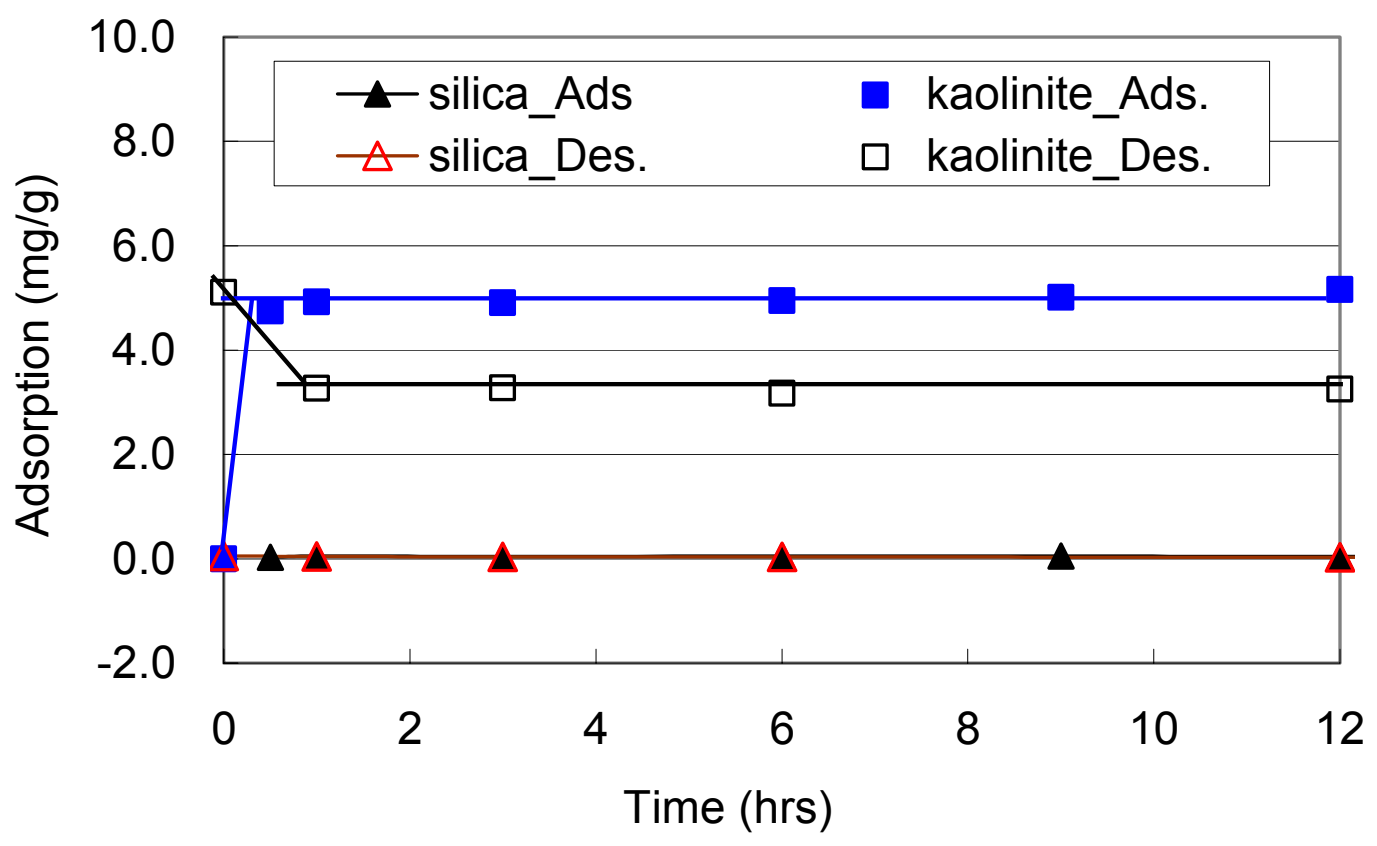

Fig. 2-6. Adsorption and desorption kinetics of CLS onto silica and kaolinite. 


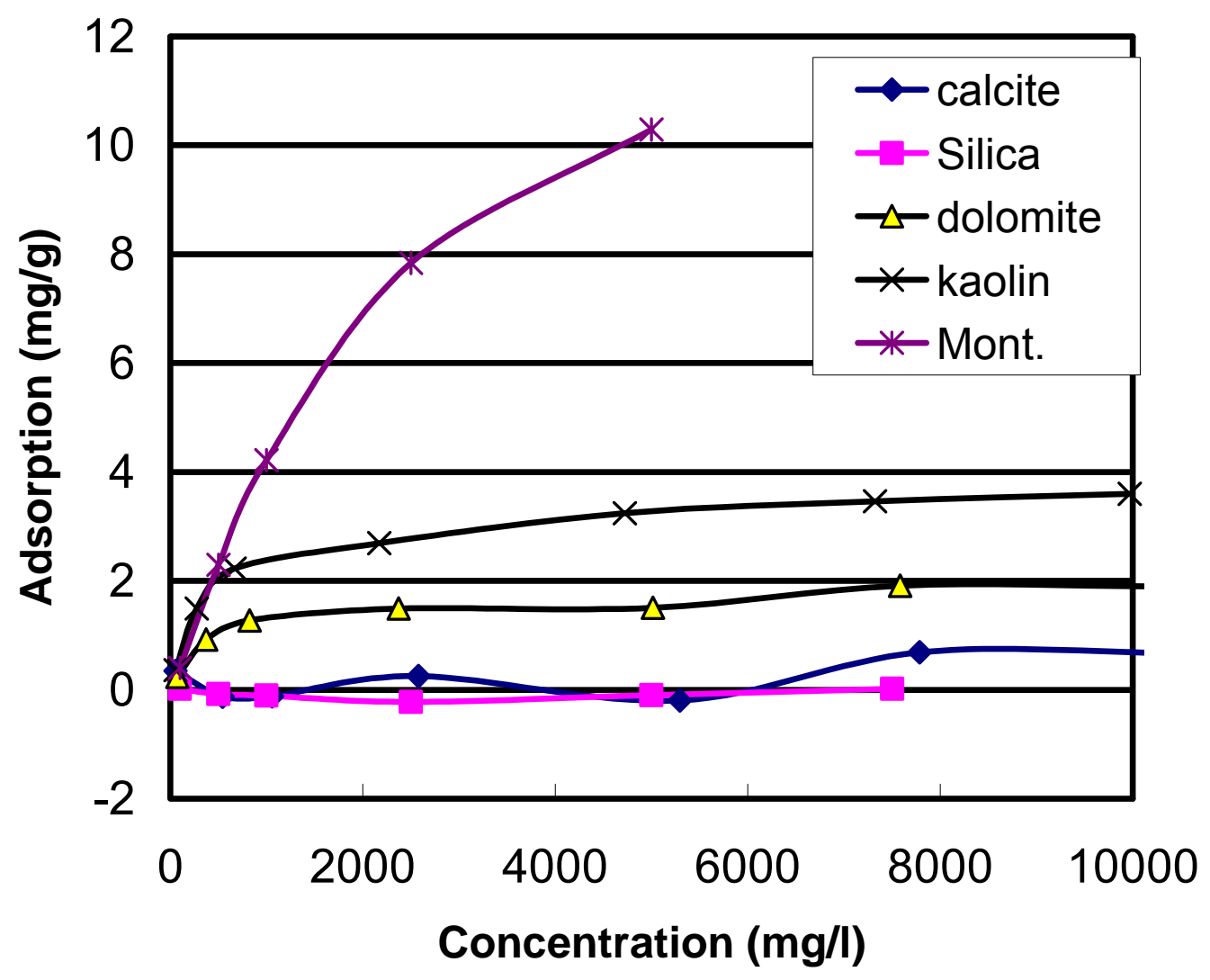

Fig. 2-7. CLS adsorption isotherm onto four minerals.

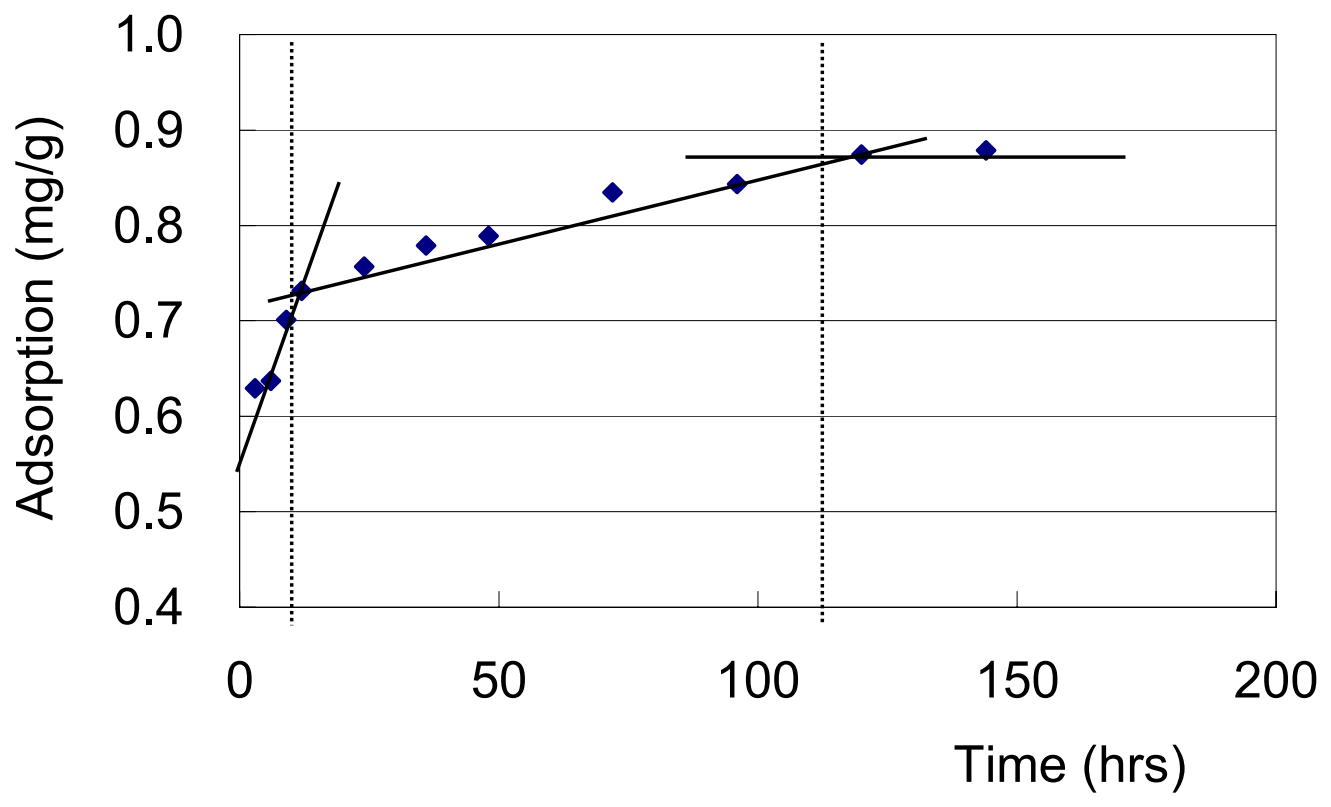

Fig. 2-8. CLS adsorption onto Berea sandstone. 


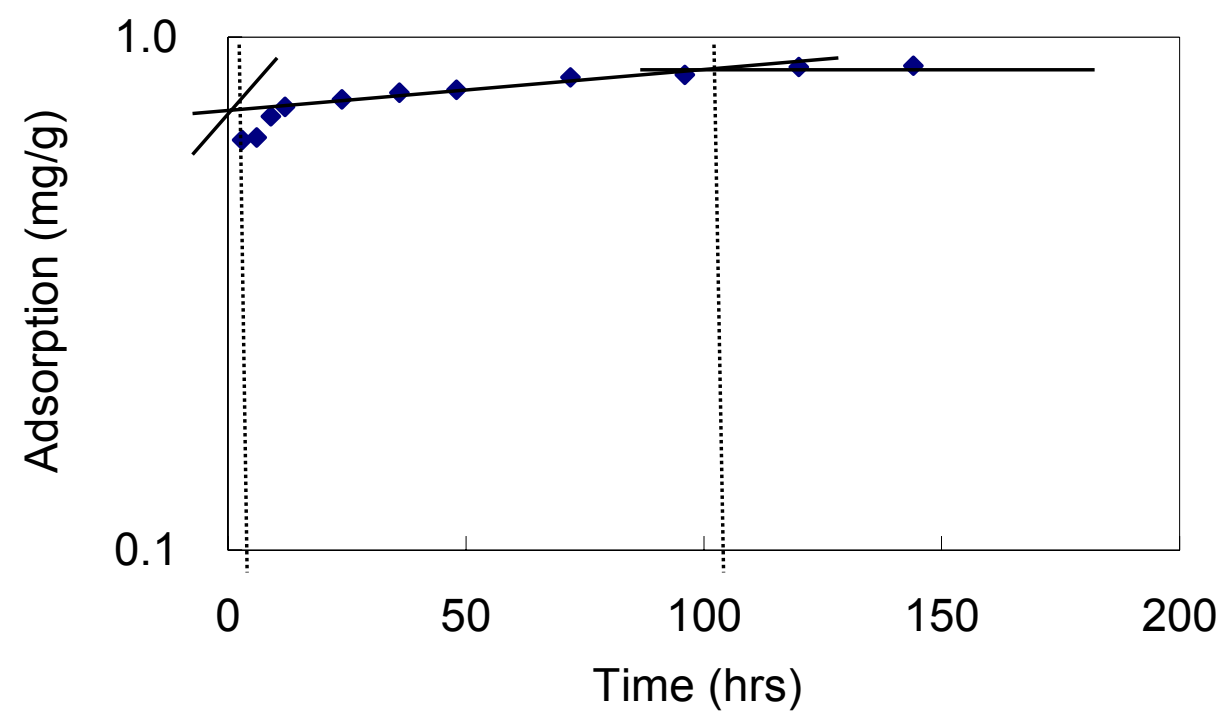

Fig. 2-9. CLS adsorption onto Berea sandstone.

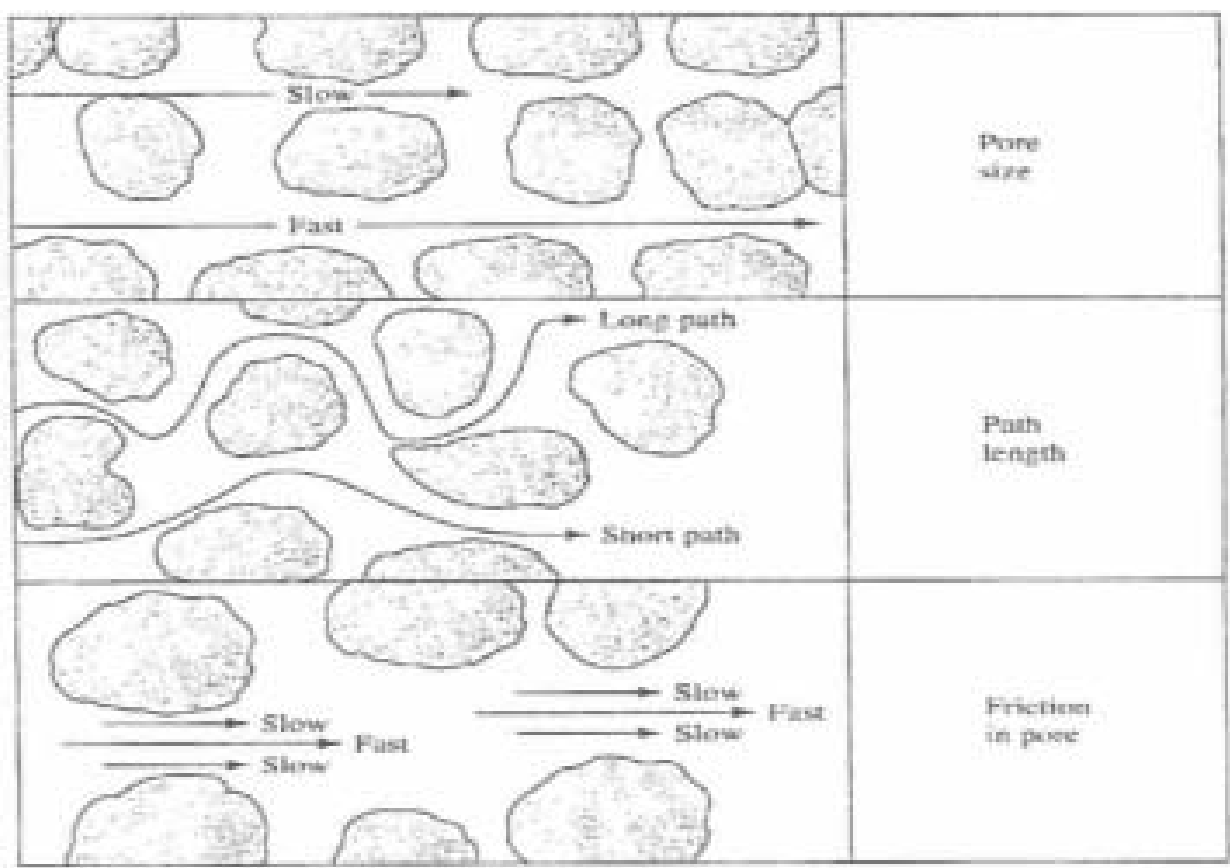

Fig. 2-10. Dispersion mechanisms of CLS through sandstone (Fetter, C.W.:Applied Hydrogeology., 2d ed., New York, Macmillan Publishing Company, 1988) 


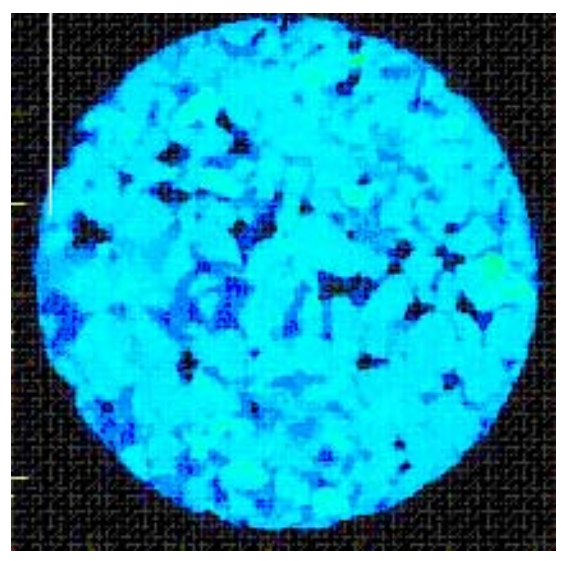

(a) Microstructure of Berea Sandstone

(http://www.bnl.gov/rocks/achievement/AGU1999/berea.html)

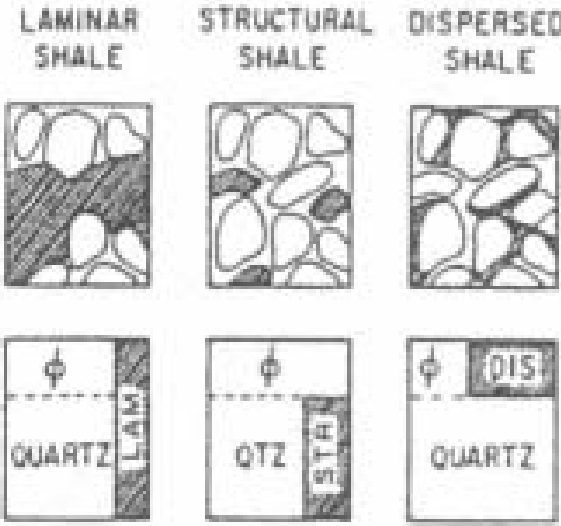

(b) Shale distribution in sandstone

Fig. 2-11. Characterization of Berea sandstone.

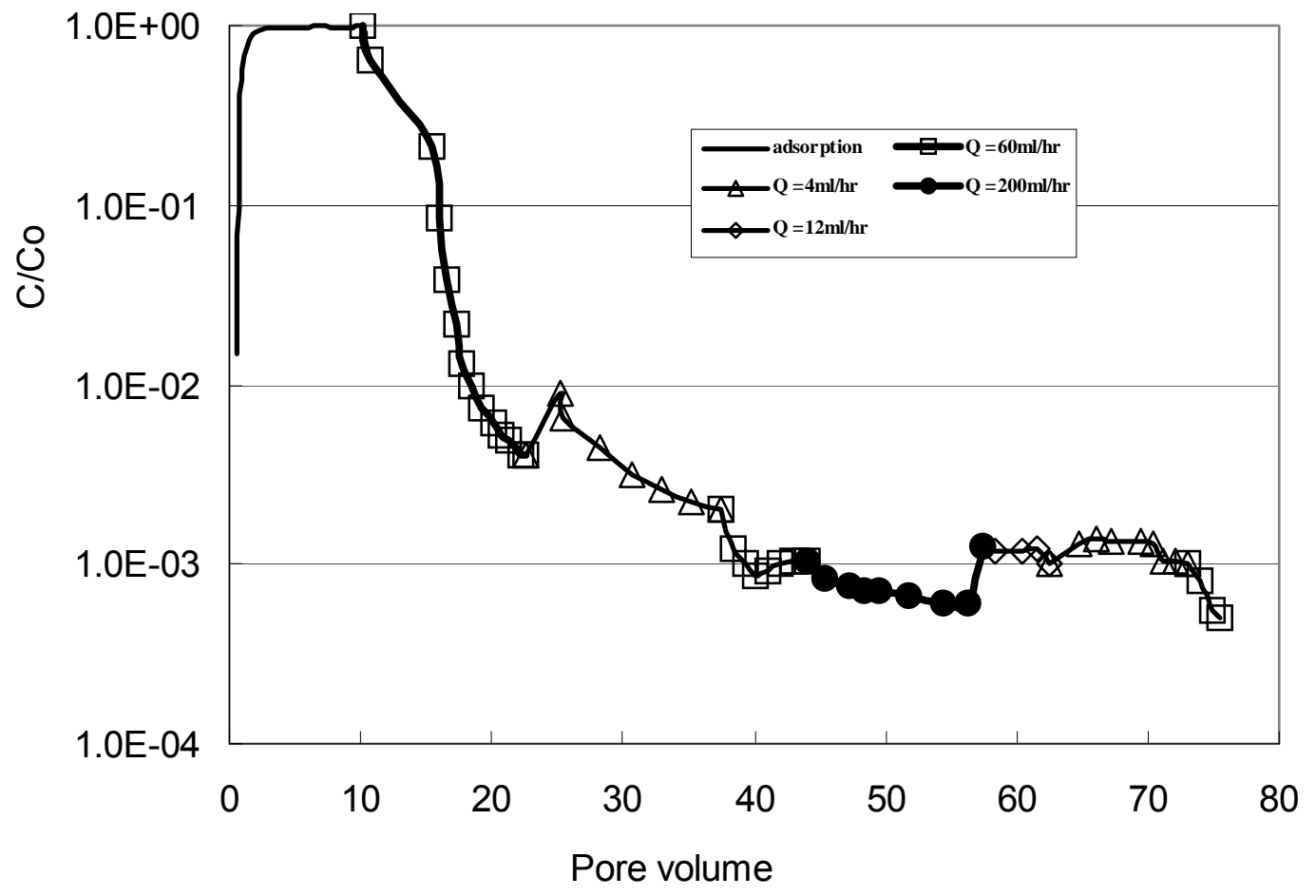

Fig. 2-12. Effect of post-flush rate on CLS desorption from Berea sandstone. 


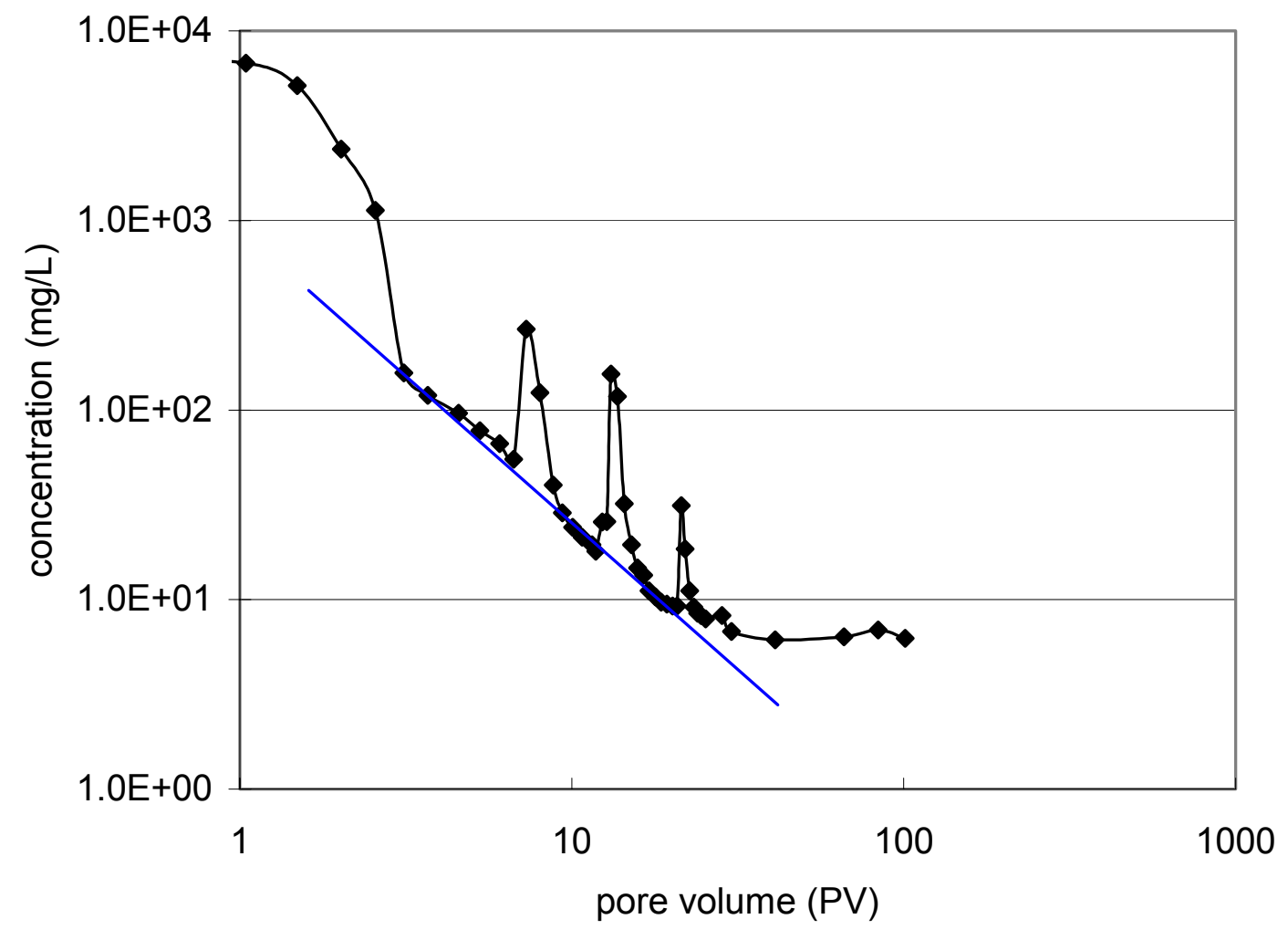

Fig. 2-13. Flow interruption experiment results.

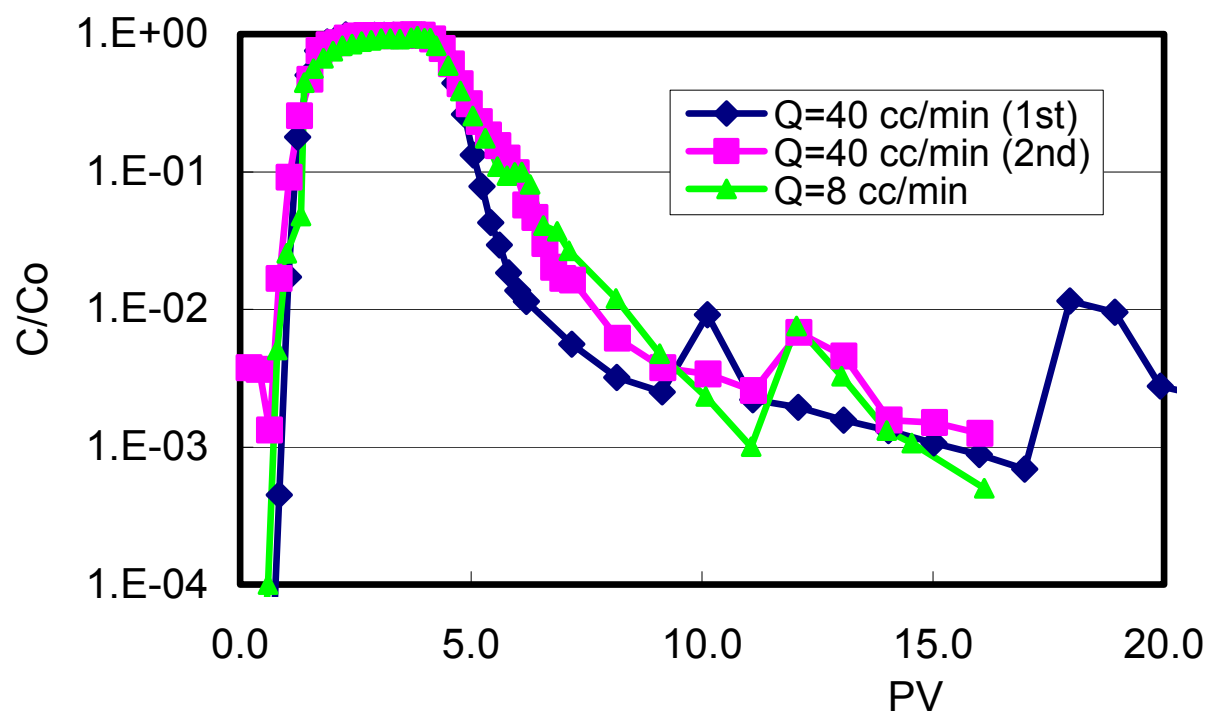

Fig. 2-14. Tracer flow through Berea sandstone. 


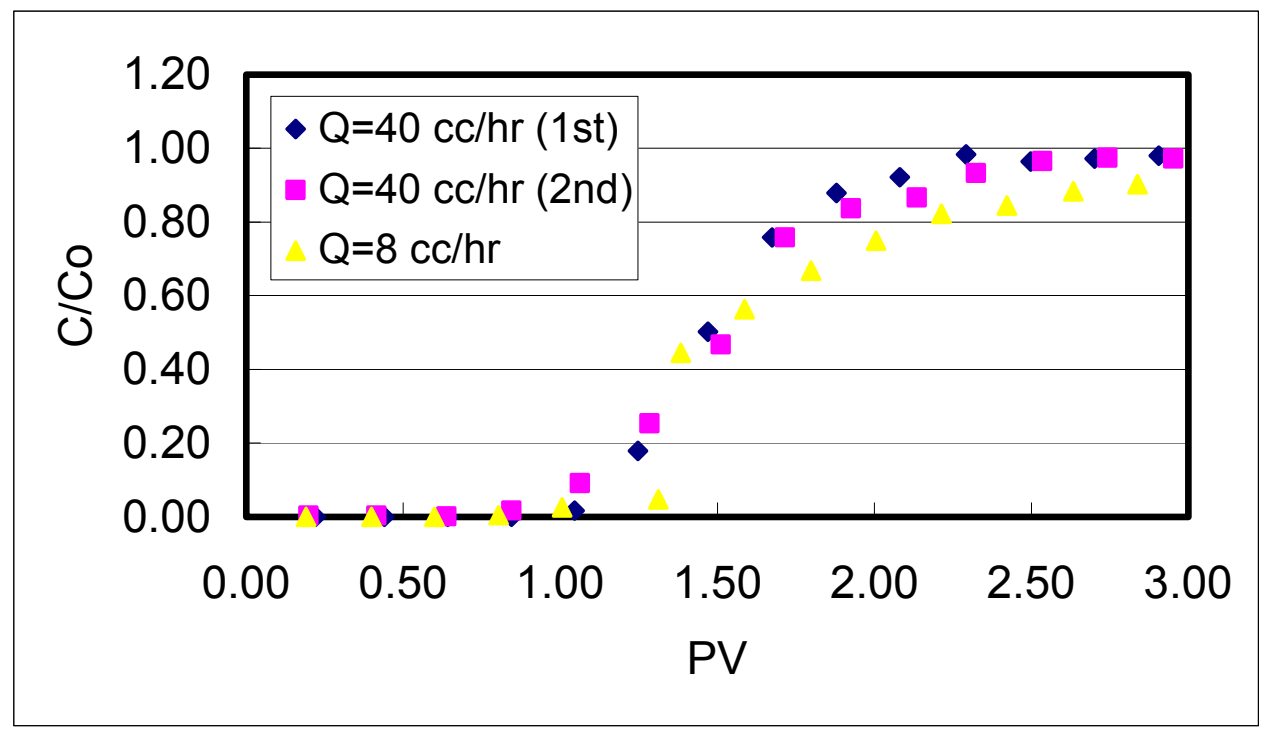

Fig. 2-15. Breakthrough curves during tracer injection.

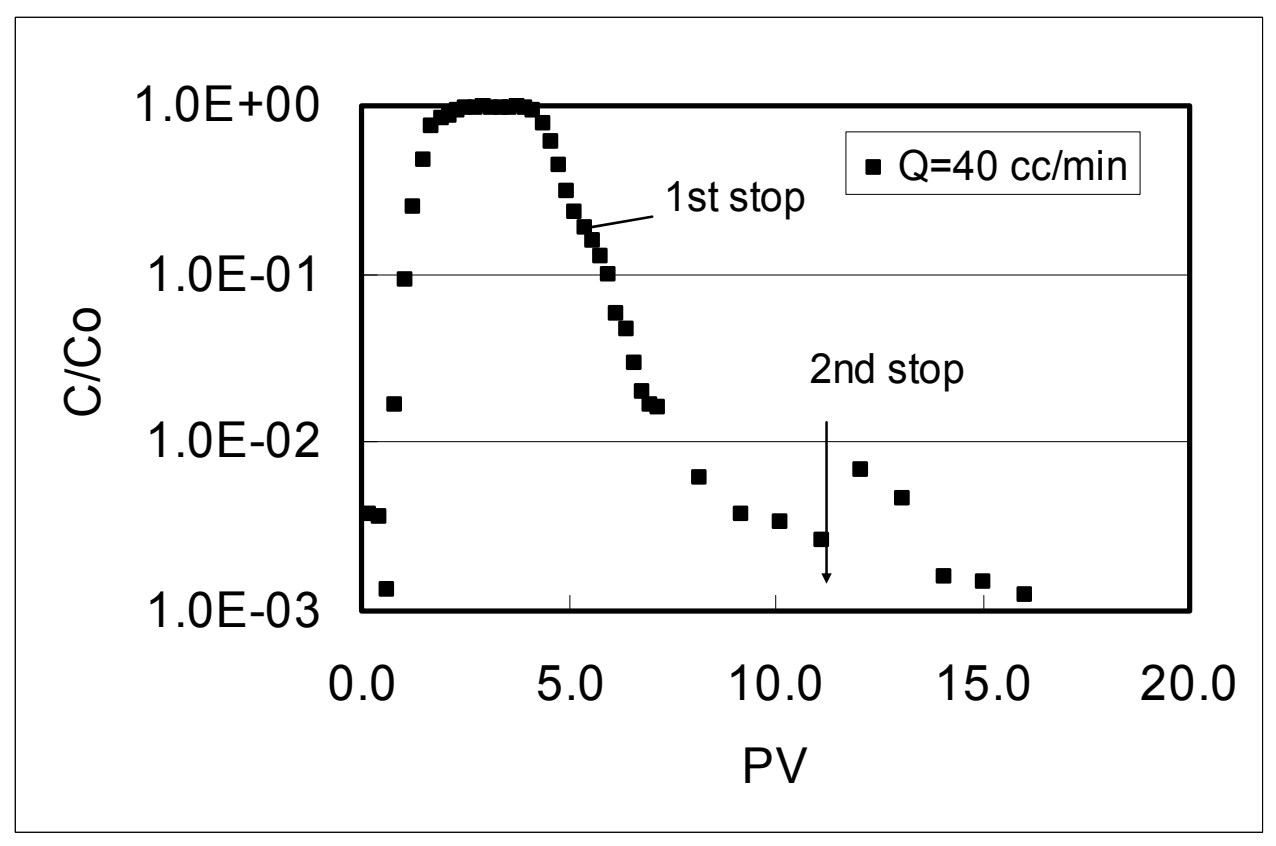

Fig. 2-16. Interruption experiment results for experiment No.2 ( stopped at 5.18 PV, 11.12PV). 


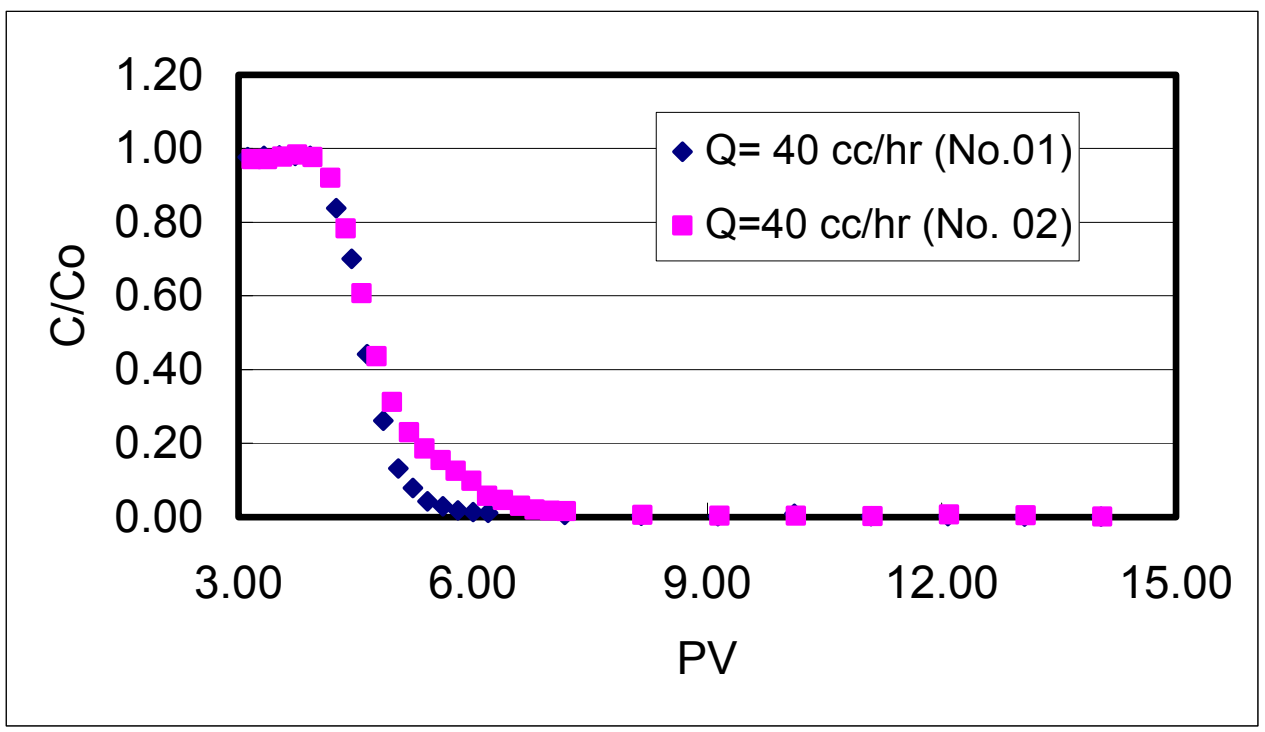

Fig. 2-17. Comparison of breakthrough curves for first two experiment during brine injection.

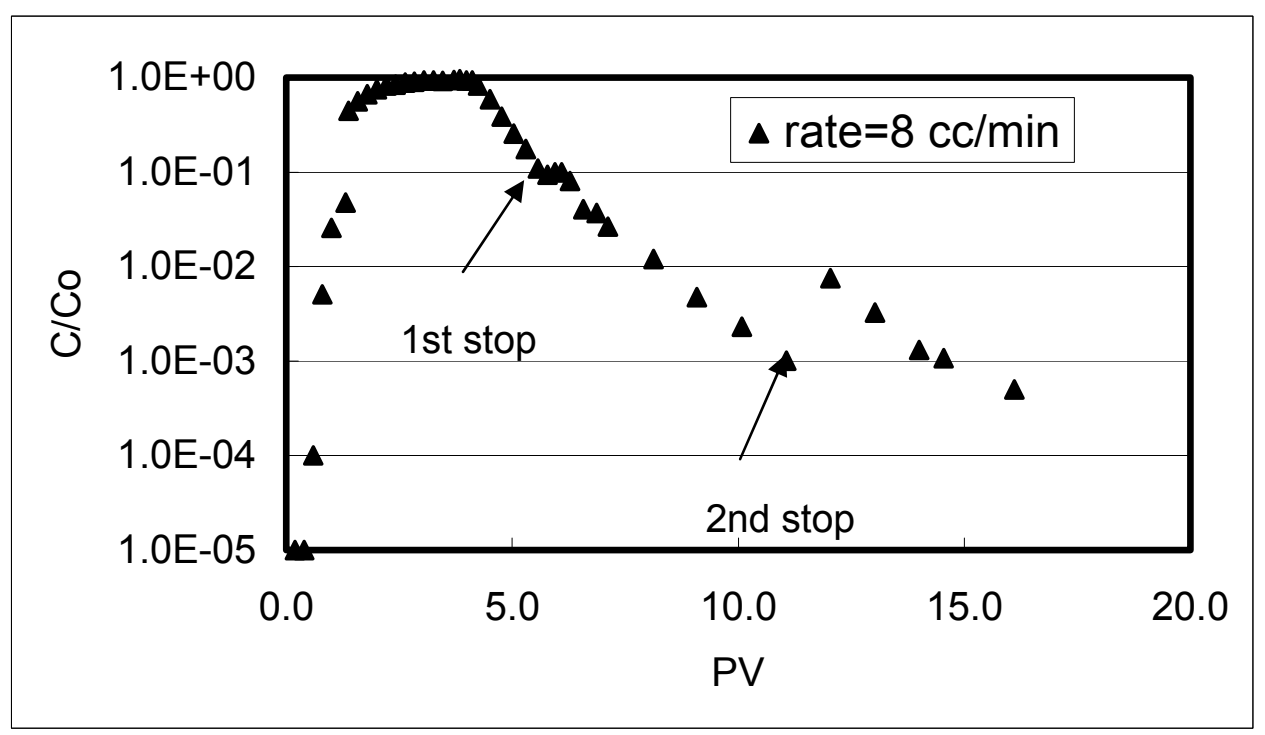

Fig. 2-18. Interruption experiment results for experiment No.3 (stopped at 5.78PV, 11.06PV). 


\title{
CHAPTER 3. SURFACTANT ADSORPTION AND DESORPTION ONTO FIVE MINERALS AND THREE POROUS MEDIA
}

\begin{abstract}
Understanding surfactant adsorption is important for surfactant application in $\mathrm{CO}_{2}$ foam flooding. Adsorption of Chaser International CD1045 ${ }^{\mathrm{TM}}$ (CD) onto five minerals and three rock types were carried out to understand surfactant adsorption behaviors and transport mechanisms through three different porous media. Batch experiments were run to determine the kinetics of the surfactant adsorption (CD, a good foam agent) onto five non-porous minerals common to the selected three porous media. Dynamic experiments were performed to determine the kinetics and equilibria of surfactant adsorption onto three porous media.
\end{abstract}

Results showed that CD adsorption and desorption onto non-porous minerals can be established in one hour. The decreasing order of the surfactant adsorption density onto the five minerals is: montmorillonite, dolomite, kaolinite, silica and calcite. Surfactant adsorption onto the three porous media took much longer than that onto the five non-porous media, and the surfactant adsorption onto Berea sandstone required a longer time than either Indiana limestone or Lockport dolomite due to its multicomponent and complex porous structure. Desorption of the surfactant from the three porous media follows exponent decline equations.

\section{Introduction}

Surfactant-based enhanced oil recovery processes have successfully demonstrated their usefulness to the petroleum industry. ${ }^{1}$ This work focuses on surfactants intended for the application of mobility control and fluid diversions caused by foam. ${ }^{2-7}$ Propagation of foam depends on the propagation of the surfactant, which is strongly affected by adsorption losses at the solid/liquid interface. Surfactant loss in a reservoir due to adsorption in porous media represents the largest consumption of chemicals in a flood, and is thus a major feature governing the economic viability 
of $\mathrm{CO}_{2}$-foam flooding. ${ }^{4}$

Numerous studies by the Gas Flooding Processes and Flow Heterogeneities Group of the PRRC have shown that CD is a good foaming agent. Tsau and Heller established CD adsorption isotherm onto limestone, Baker dolomite, and EVGSAU rock samples using distilled water and 4\% synthetic brine at room temperature. ${ }^{8,9}$ This work mainly focused on studying the adsorption features of $\mathrm{CD}$ onto three porous media and five minerals common to the five mineral.

\section{Experimental Materials}

Surfactant: CD was the surfactant used, which was identified as one of the best foaming agents in several other studies. ${ }^{1,2}$ It was supplied by Chaser International as $46.7 \mathrm{wt} \%$ active aqueous solution. Although the data sheet for this product (MSDS \#5021) states that the composition is a proprietary trade secret, it does reveal that the product contains $2.6 \%$ isopropyl alcohol $\left(\mathrm{C}_{3} \mathrm{H}_{8} \mathrm{O}\right)$. The indicator solution used to measure CD solutions states that it is a solution for anionic surfactant determination.

Dimidium Bromide-Disulphine Blue Indicator: This is a solution for anionic surfactant determination, supplied by BDH laboratory supplies. The instructions to prepare the working solution are as follows:

(1) Dilute $20 \mathrm{ml}$ of stock solution with $200 \mathrm{ml}$ of distilled water;

(2) Add $20 \mathrm{ml}$ of $2.5 \mathrm{M}$ sulfuric acid;

(3) Dilute to $500 \mathrm{ml}$ with distilled water.

Table 3-1 lists the quantity of each component required for $500 \mathrm{ml}$ of working solution.

Chloroform: The chemical formula of chloroform is $\mathrm{CHCl}_{3}$. The type used is HPLC grade and contains approximately $0.75 \%$ ethanol as a preservative. 
Brine: $2 \%$ synthetic brine was used to prepare surfactant CD solution. The brine is composed of $1.5 \% \mathrm{NaCl}$ and $0.5 \% \mathrm{CaCl}_{2}$.

Adsorbents: Five minerals were applied as adsorbents for static experiments; the source and properties of the five minerals are the same as those described in the last chapter and summarized in Table 1-1. Three types of cores were used as porous adsorbents: Berea sandstone, Indiana limestone and Lock Port dolomite. The properties of the three porous media are given in Table 3-2.

\section{Experiment Methods}

Quantitative analysis of CD solution: $\mathrm{CD}$ is a multi-component formulation that requires a special analytical procedure to measure concentration. The analytical methods suggested by the manufacturer include a two-phase hyamine titration method, a refractometric method, and a colorimetric method. All three methods can accurately determine surfactant concentration as long as the surfactant system contains only one component. Colorimetric method was used in the study.

CD solution is clear in color, so an indicator solution should be used to treat it before measurement. It is suggested by the supplier that dimidium bromide-disulphine blue stock solution be used as an indicator. The indicator solution used to measure CD concentration states that it is a solution for anionic surfactant determination. Therefore, it is assumed that CD is an anionic surfactant. The procedure for measuring CD concentration is as follows.

1. Dilute solution within range of 0 to $500 \mathrm{mg} / \mathrm{l}$ with $2 \%$ brine and shake to mix.

2. Add $1 \mathrm{~cm}^{3}$ of diluted solution to a test tube using a pipetter.

3. Add $7 \mathrm{~cm}^{3}$ of indicator solution (working solution of dimidium bromide-disulphine blue indicator) to the test tube.

4. Add $7 \mathrm{~cm}^{3}$ of chloroform (HPLC grade).

5. Cap and shake vigorously for 30 seconds to mix. There are two important notes about this step: 
a. Be sure to use a cap with a septum that is compatible with chloroform. A bad cap will swell and leak chloroform. Good caps (made of PTFE silicone) will not change shape in the presence of chloroform.

b. It is very important to shake the solution vigorously for 30 seconds. The solutions must be properly mixed or there will be variation in the absorption. This is a critical step.

6. Centrifuge at $1500 \mathrm{rpm}$ for 10 minutes.

7. Suction off the top layer with a pipette (yellow-orange in color, assumed to contain leftover dye and other components not removed by chloroform layer) and discard.

8. Save chloroform layer for analysis (clear to pink in color depending on CD composition, strong chloroform odor). Chloroform evaporates rapidly, so one should take care to minimize evaporation during operation.

9. Add $2.5-3.5 \mathrm{~cm}^{3}$ of the chloroform layer to a quartz spectrophotometer cell and analyze to measure absorption at $520 \mathrm{~nm}$. Discard solution after analyzing.

10. Calculate $\mathrm{CD}$ concentration using calibration curve.

The absorbance of CD solution prepared by $2 \%$ brine was measured by spectrophotometer, which was set to scan through a set range of wavelength from 0 to $700 \mathrm{~nm}$. CD solution has three good adsorption peaks; $295 \mathrm{~nm}, 406 \mathrm{~nm}$ and $520 \mathrm{~nm}$. Table 3-3 reports a series of absorbance measurement results of $\mathrm{CD}$ solution with different concentrations. At the peak of $406 \mathrm{~nm}$, absorbance data is very low and is not linear with CD concentration. At the peak of $295 \mathrm{~nm}$ and $523 \mathrm{~nm}$, absorbance is linear versus CD concentration at concentration below $600 \mathrm{mg} / \mathrm{l}$, as shown in Figs. 3-1 and 3-2. The standard equations are:

$$
\begin{array}{ll}
\text { At } 295 \mathrm{~nm}: \mathrm{CONC}=299.85^{*} \mathrm{ABS}-27.41 & \mathrm{R}^{2}=0.9938 \\
\text { At } 523 \mathrm{~nm}: \mathrm{CONC}=2493.77 * \mathrm{ABS}+61.10 & \mathrm{R}^{2}=0.9964
\end{array}
$$

where CONC is the CD concentration and ABS is the absorbance value of the CD solution at 295 $\mathrm{nm}$ or $523 \mathrm{~nm}$. 
In the experiments reported in this chapter, the absorbance of CD solution at the peak of $523 \mathrm{~nm}$ was selected as an evaluation standard of the CD concentration because in earlier tests lignosulfonate is mixed with $\mathrm{CD}$ as a sacrificial agent and it has an absorbance peak at $283 \mathrm{~nm}$, which is close to the $295 \mathrm{~nm} \mathrm{CD}$ peak.

CD adsorption onto five minerals: Static experiments were performed to study surfactant adsorption onto five non-porous minerals. The apparatus and setup were described in detail in Chapter 2. All static adsorption experiments were carried out at $40^{\circ} \mathrm{C}$.

CD adsorption onto three porous media: Dynamic experiments were run in sandstone, limestone and dolomite at $40^{\circ} \mathrm{C}$. Two dynamic methods, circulation and flow-through experiment described in Chapter 2, were used to study surfactant adsorption and desorption onto porous media. The amount of surfactant adsorbed is expressed as the unit: mass of CLS adsorbed per volume of rock $\left(\mathrm{mg} / \mathrm{cm}^{3}\right)$.

\section{Results and Discussion}

\section{Adsorption and Desorption Dynamic onto Five Minerals}

Static experiments were run to study $\mathrm{CD}$ adsorption and desorption onto five minerals common in reservoirs. In each experiment, 100 grams of $500 \mathrm{mg} / \mathrm{l} \mathrm{CD}$ solution was pipetted into a bottle and 25 grams of the selected mineral was weighted. The bottle and measured mineral were kept in a thermostatic bath to allow thermal equilibrium. The mineral was then mixed in the bottle with CD solution and shaken by hand vigorously for about a minute. The bottle was then placed in the thermostatic shaker bath where it was continuously shaken. A sample was taken at a designed interval. After pipetting a sample, the sample was centrifuged at $2000 \mathrm{rpm}$ for $20 \mathrm{~min}$, and the supernatant solutions were separated by decantation from the vial of the solids after gravity sedimentation. The concentration difference between the stock and the sample was used to 
evaluate the adsorption density.

Dynamic performance of $C D$ adsorption onto five minerals. Figure 3-3 shows the dynamic behavior of $\mathrm{CD}$ adsorption onto five minerals. The $\mathrm{CD}$ adsorption equilibrium onto these non-porous minerals was reached in between 0.5 and one hour. Thus, the equilibrium between the surface and CD solution is achieved in less than an hour if the solution is in immediate contact with the surface as in the non-porous case. Comparing the equilibrium time with that of CLS onto the five minerals (which is less than $0.5 \mathrm{hrs}$ ). CD takes a longer time then CLS to reach equilibrium onto the five minerals, which may be because $\mathrm{CD}$ is a multi-component formulation and competitive adsorption of the $\mathrm{CD}$ components onto these minerals increases the adsorption equilibrium time.

Figure 3-4 compares the adsorption density of $\mathrm{CD}$ onto the five minerals. The decreasing order of adsorption density is montmorillonite, dolomite, kaolinite, silica and calcite, which is different from the order of calcium lignosulfonate adsorption onto these mineral. CD adsorption density onto silica is $0.56 \mathrm{mg} / \mathrm{g}$, while CLS adsorption density onto silica is essentially zero. The equilibrium concentrations are 170, 240, 271, 361, and $389 \mathrm{mg} / \mathrm{l}$, or adsorption densities are 1.25, $1.06,0.90,0.56$, and 0.48 , respectively, for the five systems at the initial CD concentration of 500 $\mathrm{mg} / \mathrm{l}$. The CD concentration losses are 66\%, 52\%, 46\%, $27 \%$ and 23\%, respectively.

Dynamic performance of $C D$ desorption from five minerals. After completing the above adsorption experiment, a total of $50 \mathrm{~g}$ of solution (including the weight of those samples taken out for adsorption measurements) was taken out of the equilibrium adsorption system and $50 \mathrm{~g}$ of $2 \%$ brine was added to dilute the remaining CD solution in the system of adsorbate and adsorbent. A sample was taken from each bottle at a designed interval and the concentration was measured. Figure 3-5 shows the dynamic performance of CD desorption from each mineral. The equilibrium time is between three and 12 hours in each system. Figure 3-6 shows the adsorption densities of 
$\mathrm{CD}$ onto five minerals after desorption reaches equilibrium. The order of adsorption densities on the minerals is the same as that of the adsorption process. Their corresponding solution equilibrium concentrations are 72, 75, 103, 168, and $221 \mathrm{mg} / \mathrm{l}$ in solution, and 0.76, 0.59, 0.49, 0.37 , and $0.25 \mathrm{mg} / \mathrm{g}$ density on the minerals, respectively.

Comparison of CD adsorption and desorption: Figure 3-7 compares the adsorption and desorption profiles as a function of time. The equilibrium time of adsorption is much shorter than for desorption; thus the desorption rate is slower than the adsorption rate. Figure 3-8 compares relative adsorption (original adsorption density divided by the adsorption density after circulation fluid is diluted 50\%) of $\mathrm{CD}$ onto five minerals when adsorption and desorption reaches equilibrium. Thirty-five to $50 \%$ of the adsorbed material was desorbed from the surface of minerals when the concentration of equilibrium solution was diluted in half.

\section{Dynamic Performance of CD Adsorption and Desorption onto Berea Sandstone}

Dynamic performance of $C D$ adsorption onto Berea sandstone. Circulation experiments were run in core B03 (shown in Table 3-2) to study CD adsorption density as a function of time. The injection rate was $0.5 \mathrm{~cm}^{3} / \mathrm{min}$, and the initial CD concentration was $500 \mathrm{mg} / \mathrm{l}$ prepared by $2 \%$ brine. As shown in Figs. 3-9 and 3-10, it takes more than $144 \mathrm{hrs}$ to reach equilibrium. The final adsorption density is $0.570 \mathrm{mg} / \mathrm{cm}^{3}$ or $0.283 \mathrm{mg} / \mathrm{g}$. The equilibrium concentration is only $81 \mathrm{mg} / \mathrm{l}$, and $\mathrm{CD}$ concentration loss is over $84 \%$. Referring to the experimental results of CD foam creation and stability, surfactant loss is shown to reduce the effectiveness of the foam.

As shown in Fig. 3-11, the adsorption curve also can be divided by three regions. The first region is caused by the advection and dispersion of CD through porous media saturated by brine (see Chapter 2). The second region is due to the diffusion of CD between micropores or dead pores constructed by clays and macropores formed by silica. The adsorption density controlled by diffusion is about $33 \%$, which is higher than for the CLS case described in the follow chapter. The 
final region is a concentration plateau where no more $\mathrm{CD}$ was adsorbed.

Comparing the equilibrium time of $\mathrm{CD}$ onto five non-porous media, $\mathrm{CD}$ adsorption onto Berea sandstone takes a longer time to reach equilibrium. The mechanisms can also be attributed to the complex pore structures of Berea sandstone and the difference between static and dynamic experiments (dispersion effect). The detail explanation is the same as for CLS adsorption onto Berea sandstone (see Chapter 2).

Comparing equilibrium time of CLS adsorption onto Berea sandstone, CD adsorption takes a longer time to reach equilibrium. One possible explanation is that the molecular of CD is much smaller than that of CLS, so it can enter smaller micropores by diffusion where CLS cannot enter or will plug the entrance of small pores. If the explanation is reasonable, it can explain one possible mechanism for CLS as a sacrificial agent and the reason why the percent of CLS adsorption controlled by diffusion is less than the percent of CD adsorption controlled by diffusion. If no CLS is preadsorbed, $\mathrm{CD}$ will enter those micropores and it will take more time to equilibrate and a higher density of CD will be adsorbed.

Dynamic performance of $C D$ desorption from Berea sandstone. Following the adsorption experiments described above, the direct flow method was used, injecting $2 \%$ brine into the core to determine the desorption behavior of $\mathrm{CD}$. Figure 3-11 shows how much $\mathrm{CD}$ can be desorbed from the Berea sandstone at different pore volume injections of $2 \%$ brine. The desorption rate of CD is very slow. Only about $5 \%$ of CD was desorbed when $1 \mathrm{PV}$ of brine was injected in the pore, and about $20 \%$ was desorbed when injection volume of brine reached $10 \mathrm{PV}$. At the end of the desorption experiment about 115 PV brine was injected, with about $30 \%$ of the adsorbed CD, 0.17 $\mathrm{mg} / \mathrm{cm}^{3}$, remaining in the core.

Figure 3-12 shows the adsorption density as a function of post-flush time for the desorption 
experiment. The plot of time versus log adsorbed has a good linear relationship with $\mathrm{R}^{2}$ of 0.9898 , and the following equation fit:

$$
q=0.5242 \mathrm{e}^{-0.016 \mathrm{t}}
$$

where $\mathrm{q}$ is the adsorption density $\left(\mathrm{mg} / \mathrm{cm}^{3}\right), \mathrm{t}$ is the time $(\mathrm{hrs})$.

\section{Dynamic Performance of CD Adsorption and Desorption onto Limestone}

Dynamic performance of $C D$ adsorption onto limestone. Circulation experiments were run in core L01 in Table 3-2 to study the dynamic performance of CD adsorption onto limestone. The injection rate was $0.25 \mathrm{~cm}^{3} / \mathrm{min}$, and the initial CD concentration was $500 \mathrm{mg} / 1$ prepared in $2 \%$ brine. Figures 3-13 and 3-14 give the adsorption densities with time. The adsorption equilibrium was reached in $12 \mathrm{hrs,}$ which is longer than the equilibrium time of $\mathrm{CD}$ adsorption onto non-porous calcite minerals, but much shorter than the equilibrium time of $\mathrm{CD}$ adsorption onto Berea sandstone. The adsorption density was determined to be about $0.416 \mathrm{mg} / \mathrm{cm}^{3}$, or $0.178 \mathrm{mg} / \mathrm{g}$. The equilibrium concentration was $107 \mathrm{mg} / \mathrm{l}$, and CD concentration loss was about 78\%. Similarly, the adsorption would decrease effectiveness of CD foam.

Comparing Fig. 3-14 for limestone with Fig. 3-10, the adsorption controlled by diffusion is not obvious. Only two regions are clearly shown: the region caused by advection and dispersion of CD and the plateau region. X-ray diffraction data show that the Indiana limestone is made up predominantly of calcite $(99 \%)$ with a small amount of quartz $(1 \%){ }^{10}$ The pore constructed by calcite is relative simple, minimizing the impact of diffusion in this case.

Dynamic performance of $C D$ desorption from limestone. Following the above described adsorption experiments, $2 \%$ brine was injected into the core and the effluent was collected to analysis $\mathrm{CD}$ concentration change during the desorption process. Figure 3-15 represents CD adsorption densities as a function of injection volumes of postflush fluid. As shown in the figure, about $5 \%$ of $\mathrm{CD}$ was desorbed when $1 \mathrm{PV}$ of brine was injected in the core, and about $22 \%$ had 
been desorbed after the injection of $10 \mathrm{PV}$ of brine. At the end of the desorption experiment (about $105 \mathrm{PV}$ brine had been injected), there was about $6 \%$ of adsorbed $\mathrm{CD}, 0.027 \mathrm{mg} / \mathrm{cm}^{3}$ remaining adsorbed.

Figure 3-16 shows the plot of adsorption density and flushing time during desorption. CD adsorption density also shows a good linear relationship with time versus log of CD concentration adsorbed. The $\mathrm{R}^{2}$ is 0.9873 and the fit equation is:

$$
\mathrm{q}=0.4212 \mathrm{e}^{-0.024 \mathrm{t}}
$$

From Equations (3-1) and (3-2), it is found that the CD desorption shows the same rule, both of them following the rule of exponential decline, similar to the rate decline curve for oil production. If this is shown to be a general rule, the model will be used to predict desorption performance. Further study will demonstrate whether this relationship is common to all CD desorption from porous media.

\section{Dynamic Performance of CD Adsorption and Desorption onto Dolomite}

Dynamic performance of CD adsorption onto dolomite. A similar circulation experiment was run in core D01 (shown in Table 3-2) to study the dynamic performance of CD adsorption onto dolomite. As shown in Figs. 3-17 and 3-18, the equilibrium time is about 24 hrs, a little longer than in limestone system but still shorter than in the Berea sandstone system. The equilibrium concentration is $331 \mathrm{mg} / \mathrm{l}$, and the corresponding adsorption density is $0.212 \mathrm{mg} / \mathrm{cm}^{3}$ or 0.091 $\mathrm{mg} / \mathrm{g}$. The concentration loss was about $34 \%$.

X-ray diffraction data show that dolomite is made up predominantly of the mineral dolomite (about 98\%) and trace amounts of illite, quartz and chlorite. ${ }^{10}$ This may be the reason why the equilibrium time of CD adsorption onto dolomite is below 24 hrs because it may have a relatively simple pore structure. It seems that it is reasonable to divide dolomite adsorption curve into two regions, shown in Fig. 3-18. The mechanisms controlling two regions are the same as the CD adsorption on limestone. There is a small transition region that could be diffusion, but is supposed 
to be a short tail of the advection region.

Dynamic performance of $C D$ desorption from dolomite. After the above adsorption experiment, the fluid in the mixing bottle was changed to $2 \%$ brine. A circulation experiment was run to determine the dynamic performance of $\mathrm{CD}$ desorption from dolomite. The total circulation fluid volume was still 100 grams, including the CD solution in the core, the tube and the brine in the mixing bottle. Figures 3-19 shows CD concentration change with time during the desorption process. The CD concentration first shows a rapid increase since the brine in mixing bottle was mixed with the CD left in the core and tube and some adsorbed CD was desorbed from the surface of the core. Then it shows a small increase due to the $\mathrm{CD}$ desorption from the surface and relative small pores in the core. Finally, a constant concentration is shown, indicating equilibrium adsorption was reached at a new $\mathrm{CD}$ concentration. The new equilibrium concentration is about $140 \mathrm{mg} / \mathrm{l}$.

Figure 3-20 shows a semilog plot of $\mathrm{CD}$ adsorption density with postflush time during $\mathrm{CD}$ desorption process. Similar to the adsorption curve of CD onto dolomite, the plot can be divided into two regions. The corresponding mechanisms controlling different regions are the same as the explanations on CD adsorption on limestone.

\section{Comparison of CD Adsorption Density onto Three Porous Media}

Figure 3-21 compares the adsorption density of CD onto Berea sandstone, Indian limestone and dolomite. Figure 3-22 shows the corresponding concentration loss due to $\mathrm{CD}$ adsorption onto these cores. Table 3-4 lists the three dynamic adsorption experiment results, each starting with $500 \mathrm{mg} / \mathrm{l}$ CD solution. Berea sandstone shows the maximum adsorption density and the maximum adsorption loss.

Comparing the adsorption densities of CD onto dolomite and limestone for dynamic adsorption 
and those for static adsorption experiment shows different trends. For the dynamic experiment, the adsorption density of $\mathrm{CD}$ onto limestone is higher than that of $\mathrm{CD}$ onto dolomite, while the trend is opposite for static experiments. Again, this may be due to their different surface areas. The surface area will be measured to see if this helps explain the results.

\section{Conclusions}

1. CD adsorption and desorption onto non-porous minerals can be established in an hour. The decreasing order of $\mathrm{CD}$ adsorption density onto five minerals is montmorillonite, dolomite, kaolinite, silica and calcite.

2. CD adsorption onto three porous media took much longer than that onto five non-porous media. $\mathrm{CD}$ adsorption onto Berea sandstone had the longest time among the three system of $\mathrm{CD}$ and porous media due to its complex pore structure.

3. The curves of $\mathrm{CD}$ adsorption onto Berea sandstone can be divided into three regions, corresponding to three different adsorption mechanisms. The curves of $\mathrm{CD}$ adsorption onto limestone and dolomite appear to have only two regions, adsorption controlled by diffusion is not important for these two systems.

4. Direct flow experiments show that desorption of CD from Berea sandstone and limestone follows exponent decline rule, similar to the rate change for oil production.

5. CD surfactant loss due to adsorption onto rock is from $34 \%$ to $84 \%$ in our experiments at the initial CD concentration of $500 \mathrm{mg} / \mathrm{l}$.

\section{References}

1. Schramm, L.: Foams: Fundamentals and Applications in the Petroleum Industry. American Chemistry Society, Washington, DC, 1994.

2. Tsau, J-S. and Heller, J. "Evaluation of Surfactants for $\mathrm{CO}_{2}$-Foam Mobility Control," paper SPE 24013 presented at the 1992 SPE Permian Basin Oil and Gas Recovery 
Conference, Midland, March 18-20.

3. Kuhlman, M., Lau, H., and Falls, A.: "Surfactant Criteria for Successful Carbon Dioxide Foam in Sandstone Reservoirs," SPERE 3 (2000), 35.

4. Grigg, R., Tsau, J-S. and Martin, F.: "Cost Reduction and Injectivity Improvements for CO2 Foams for Mobility Control," paper SPE 75178 presented at the 2002 SPE/DOE Improved Oil Recovery Symposium, April 13-17.

5. Mannhardt, K., Schramm, L., and Novosad, J.: "Effect of Rock Type and Brine Composition on Adsorption of Two Foam-Forming Surfactants ," paper SPE 20463 presented at the 1990 SPE Annual Technical Conference and Exhibition, New Orleans, Sept. 23-26.

6. Smith, D.: Surfactant-Based Mobility Control. American Chemical Society, 1988.

7. Kuhlman, M., Falls, A., Hara, S. Monger-McClure, T., and Borchardt, J. : " $\mathrm{CO}_{2}$ Foam With Surfactants Used Below Their Critical Micelle Concentrations," SPERE (1992) 7, 445.

8. Tsau, J-S. and Heller, J.: "Evaluation of Surfactants for $\mathrm{CO}_{2}$-Foam Mobility Control," paper SPE 24013 presented at the 1992 Permian Basin Oil and Gas Recovery Conference, Midland, March 18-20.

9. Tsau, J-S. et al.: " $\mathrm{CO}_{2}$ Foam Field Verification Pilot Test at EVGSAU: Phase IIIA: Surfactant Performance Characterization and Quality Assurance," paper SPE 27785 presented at the 1994 SPE/DOE Symposium on Improved Oil Recovery, Tulsa, April 17-21.

10. Churcher L. et al.: "Rock Properties of Berea Sandstone, Baker Dolomite, and Indiana Limestone," paper SPE21044 presented at the 1991 SPE International Symposium on Oilfield Chemistry, Anaheim, February 20-22. 
Table 3-1. Summary of Mixture Components for Making Indicator Solution

\begin{tabular}{|c|c|c|}
\hline Component & Compositions & Amount in 500mL (m) \\
\hline $\begin{array}{c}\text { Dimidium Bromide-Disulphine Blue } \\
\text { Stock Solution }\end{array}$ & $4 \%$ & 20 \\
\hline 2.5M Sulfuric Acid & $4 \%$ & 20 \\
\hline Distilled Water & $92 \%$ & 460 \\
\hline Total & $100 \%$ & 500 \\
\hline
\end{tabular}

Table 3-2. Parameters of Three Porous Media

\begin{tabular}{|c|l|c|c|c|c|c|c|c|}
\hline Code & Core & $\begin{array}{c}\text { Length } \\
(\mathrm{cm})\end{array}$ & $\begin{array}{c}\text { Diameter } \\
(\mathrm{cm})\end{array}$ & $\begin{array}{c}\text { Bulk } \\
\text { volume } \\
(\mathrm{cm} 3)\end{array}$ & $\begin{array}{c}\text { Weight } \\
(\mathrm{g})\end{array}$ & $\begin{array}{c}\text { Pore } \\
\text { Volume }\end{array}$ & $\begin{array}{c}\text { Porosity } \\
(\%)\end{array}$ & $\begin{array}{c}\text { Permeability } \\
(\mathrm{md})\end{array}$ \\
\hline B03 & Sandstone & 6.20 & 3.81 & 70.69 & 142.21 & 11.86 & 16.78 & 224.12 \\
\hline L01 & Limestone & 7.90 & 3.81 & 90.07 & 210.23 & 6.64 & 7.32 & 21.89 \\
\hline D01 & Dolomite & 5.70 & 3.81 & 64.99 & 150.47 & 5.01 & 7.71 & 24.72 \\
\hline
\end{tabular}

Table 3-3. Peak Absorbance of CD Solution

\begin{tabular}{|c|c|c|c|}
\hline \multirow{2}{*}{ Conc. } & \multicolumn{3}{|c|}{ Absorbance at Peak } \\
\cline { 2 - 4 } & $295 \mathrm{~nm}$ & $406 \mathrm{~nm}$ & $523 \mathrm{~nm}$ \\
\hline 10 & 0.1017 & -0.0333 & -0.0227 \\
\hline 50 & 0.2405 & -0.0315 & -0.0095 \\
\hline 100 & 0.4135 & -0.0300 & 0.0125 \\
\hline 150 & 0.5970 & -0.0290 & 0.0340 \\
\hline 200 & 0.8120 & -0.0200 & 0.0600 \\
\hline 250 & 0.8750 & -0.0270 & 0.0730 \\
\hline 300 & 1.0380 & -0.0250 & 0.0910 \\
\hline 400 & 1.5090 & -0.0190 & 0.1340 \\
\hline 500 & 1.7400 & -0.0190 & 0.1750 \\
\hline 600 & 2.1560 & 0.0130 & 0.2160 \\
\hline
\end{tabular}

Table 3-4. 500mg/l CD Adsorption onto Three Porous Media

\begin{tabular}{|l|c|c|c|}
\hline Core & $\begin{array}{c}\text { Adsorption density } \\
\left(\mathrm{mg} / \mathrm{cm}^{3}\right)\end{array}$ & $\begin{array}{c}\text { Equilibrium Conc. } \\
(\mathrm{mg} / \mathrm{L})\end{array}$ & $\begin{array}{c}\text { Conc. Loss } \\
(\%)\end{array}$ \\
\hline Berea & 0.570 & 81 & 84 \\
\hline Limestone & 0.416 & 107 & 78 \\
\hline Dolomite & 0.212 & 331 & 34 \\
\hline
\end{tabular}




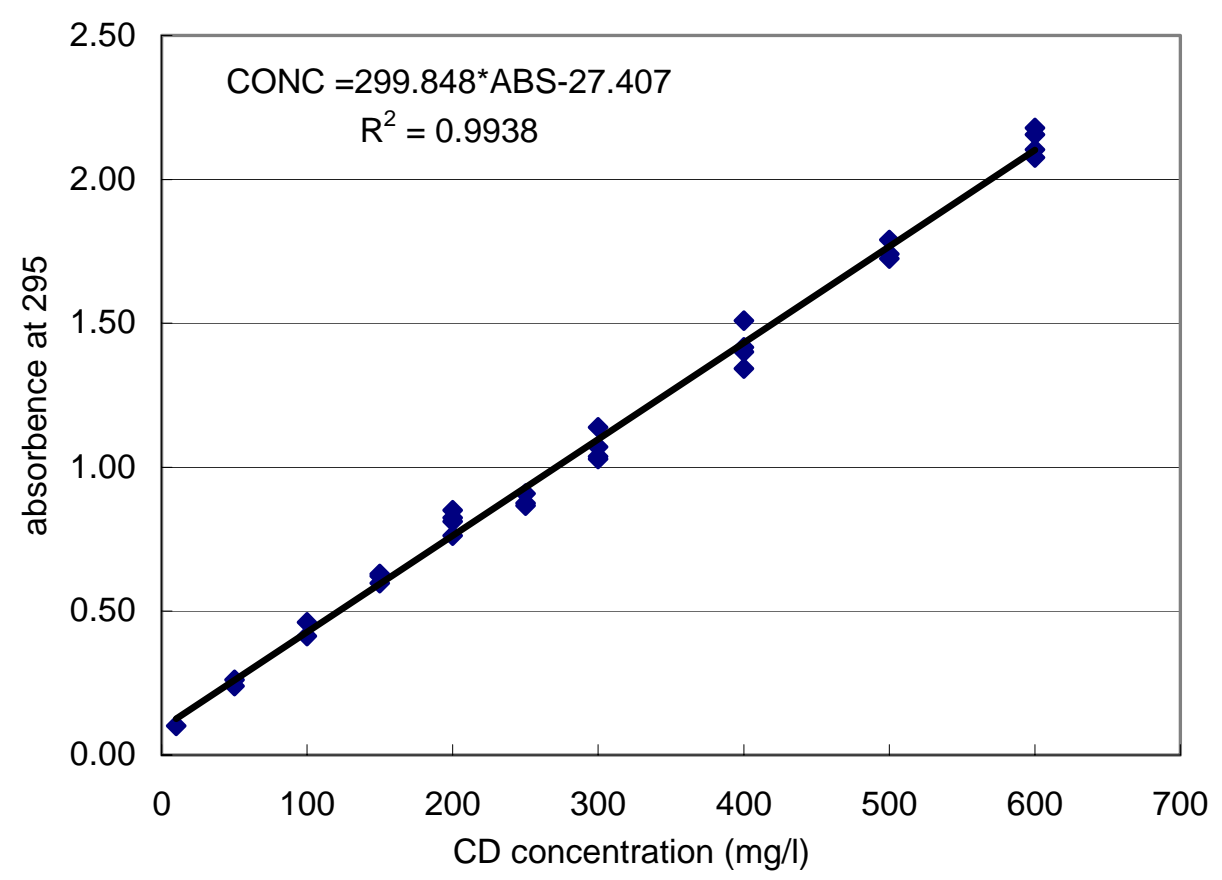

Fig. 3-1. standard curve of CD at 295nm.

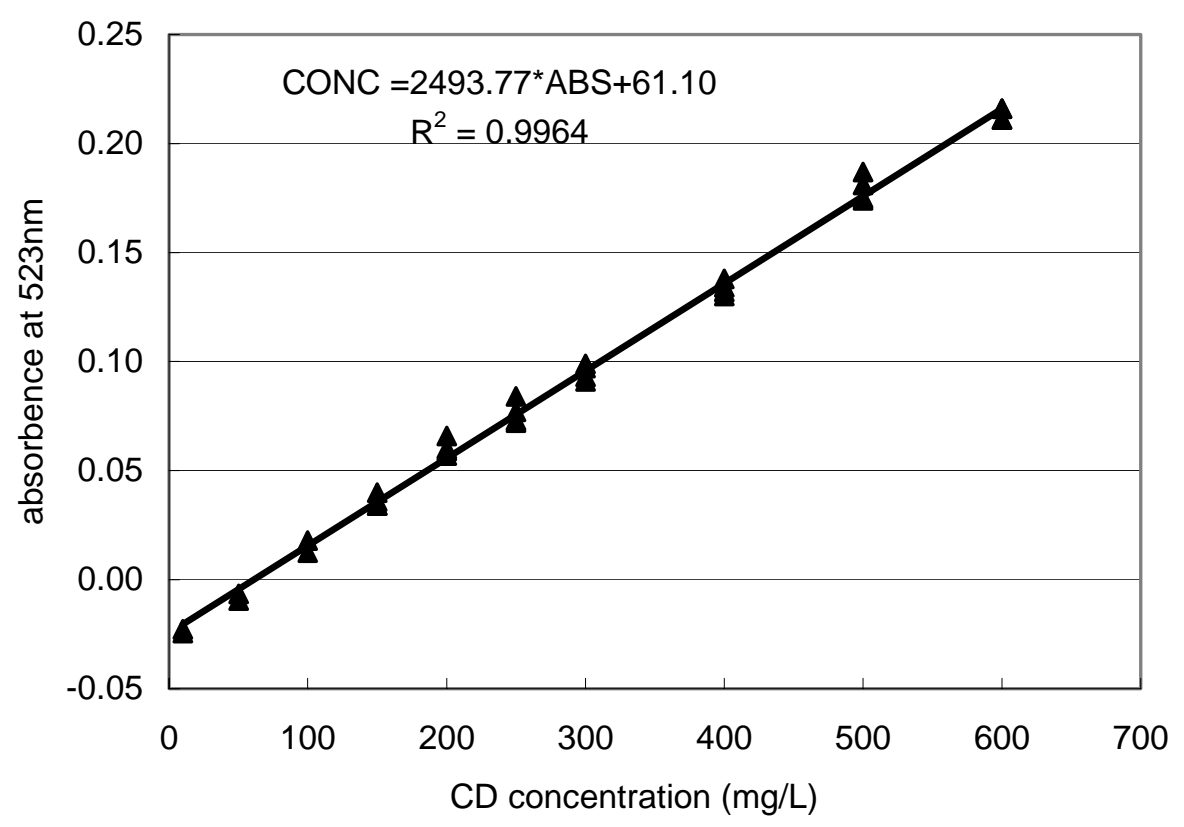

Fig. 3-2. Standard curve of CD at $523 \mathrm{~nm}$. 


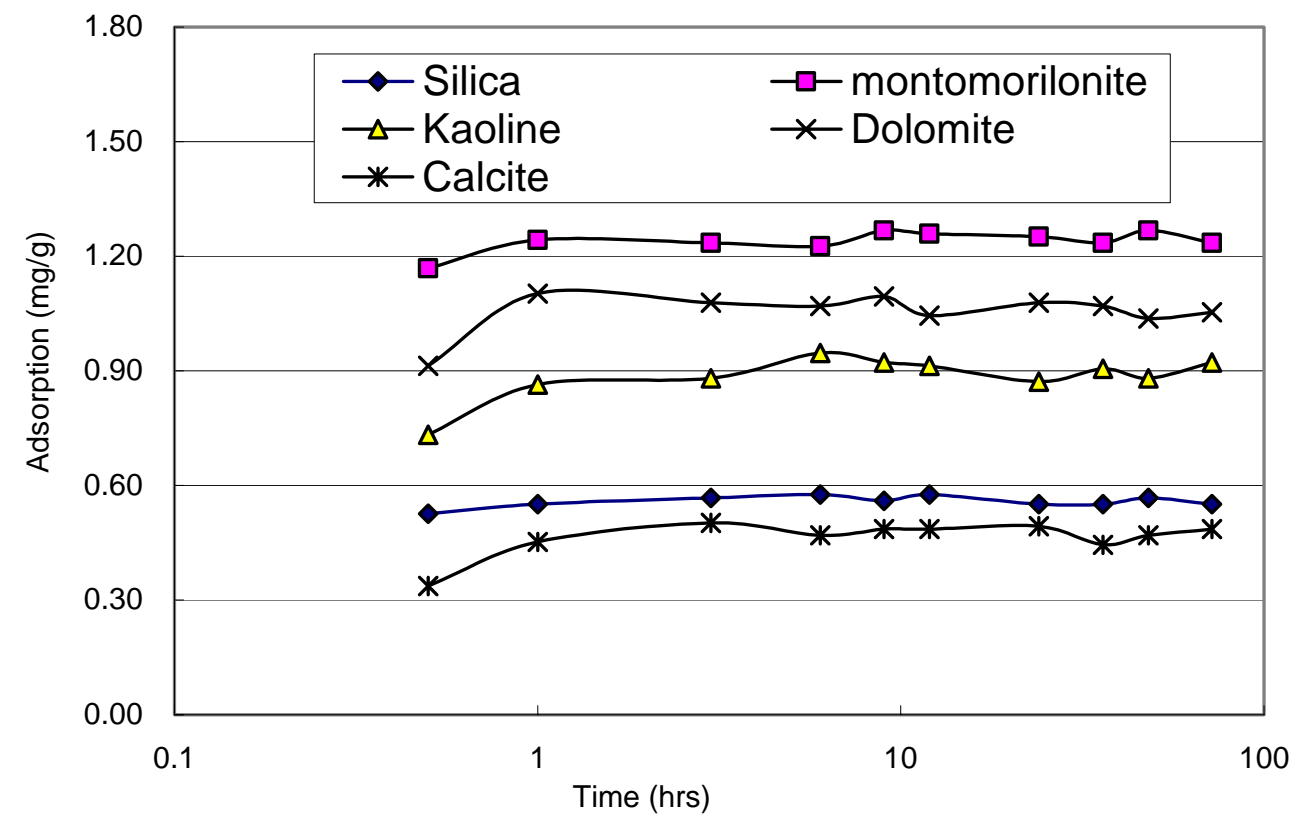

Fig. 3-3. CD adsorption process onto five adsorbents.

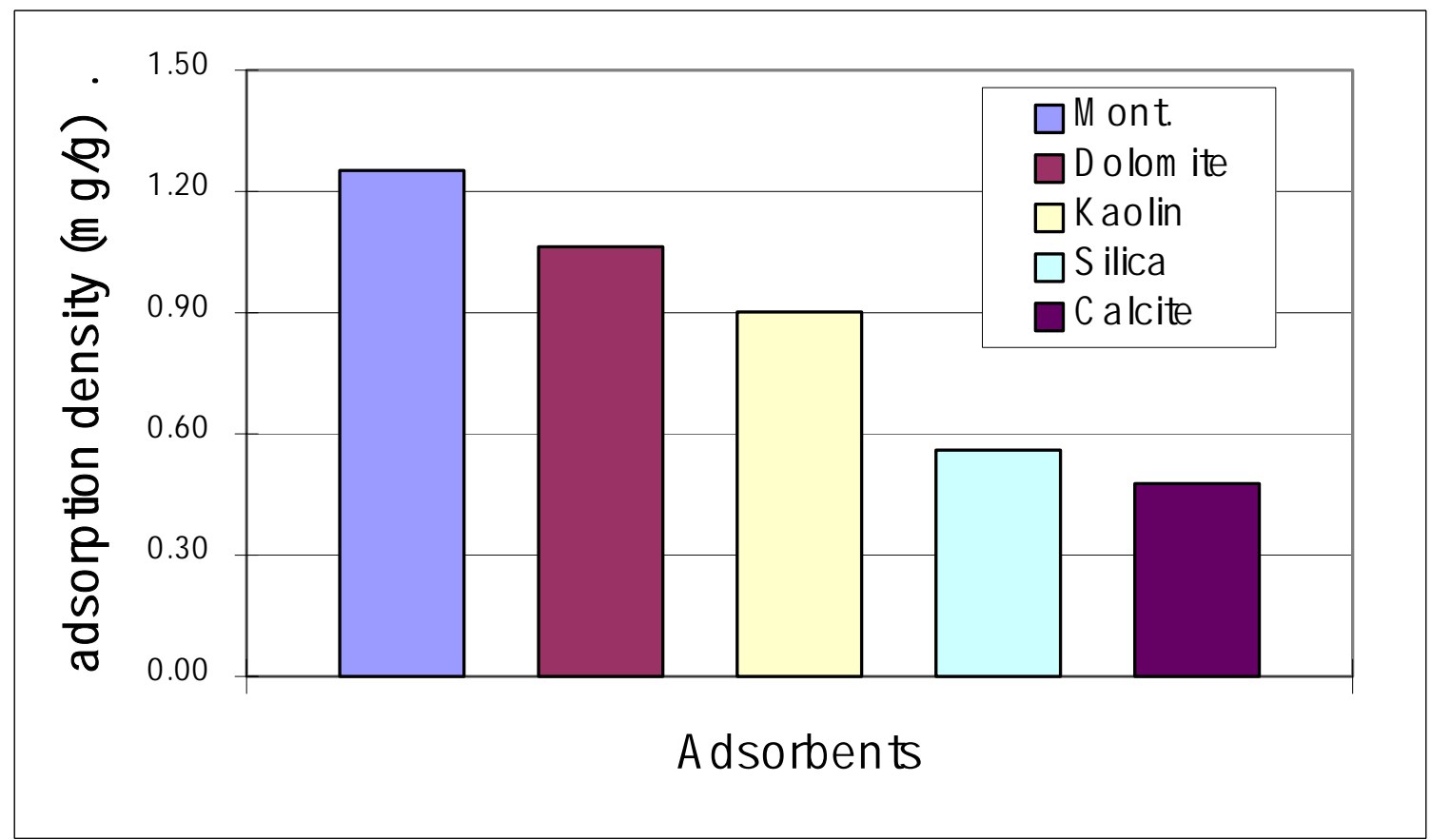

Fig. 3-4. Adsorption density of CD onto five minerals (adsorption results). 


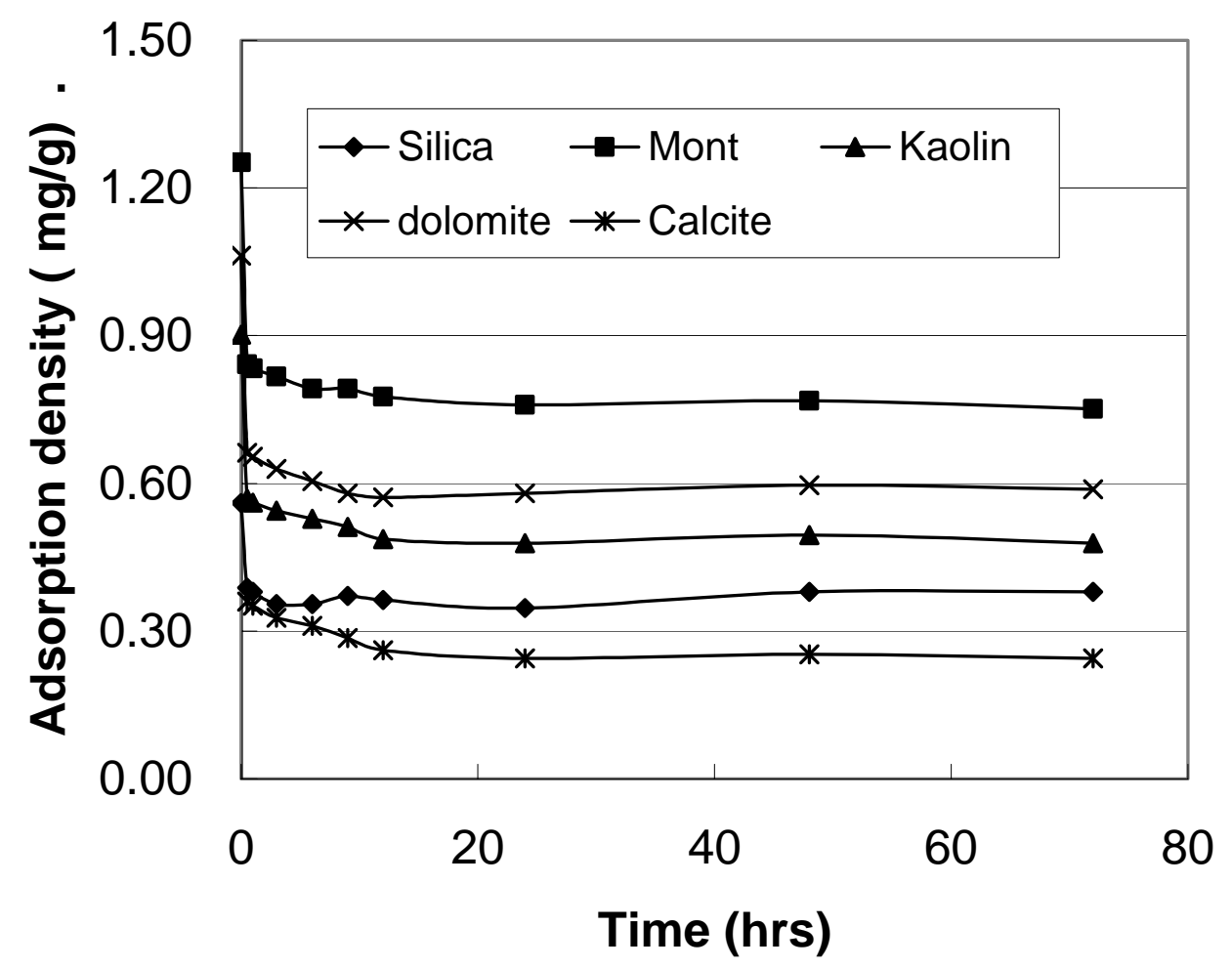

Fig. 3-5. CD desorption from five minerals as a function of time.

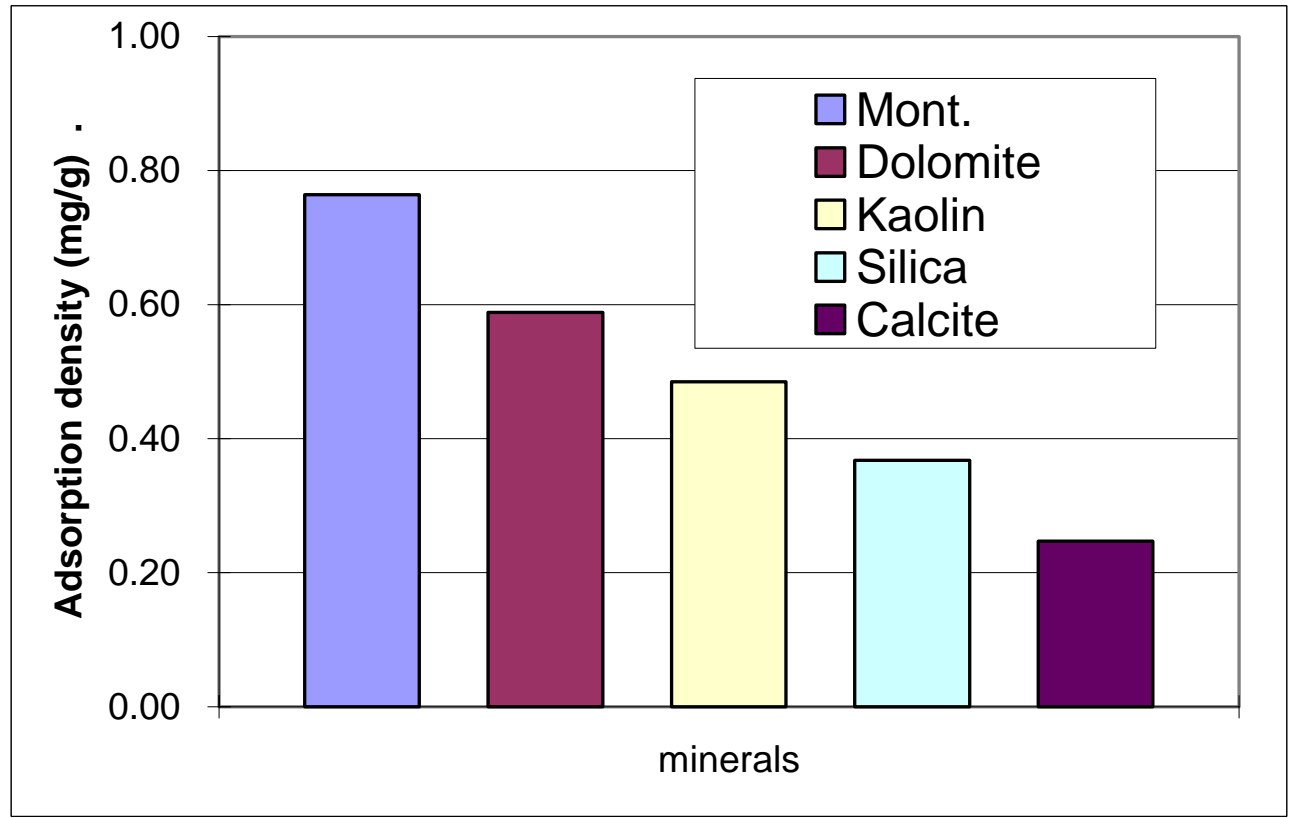

Fig. 3-6. Adsorption density of CD onto five minerals (desorption results). 


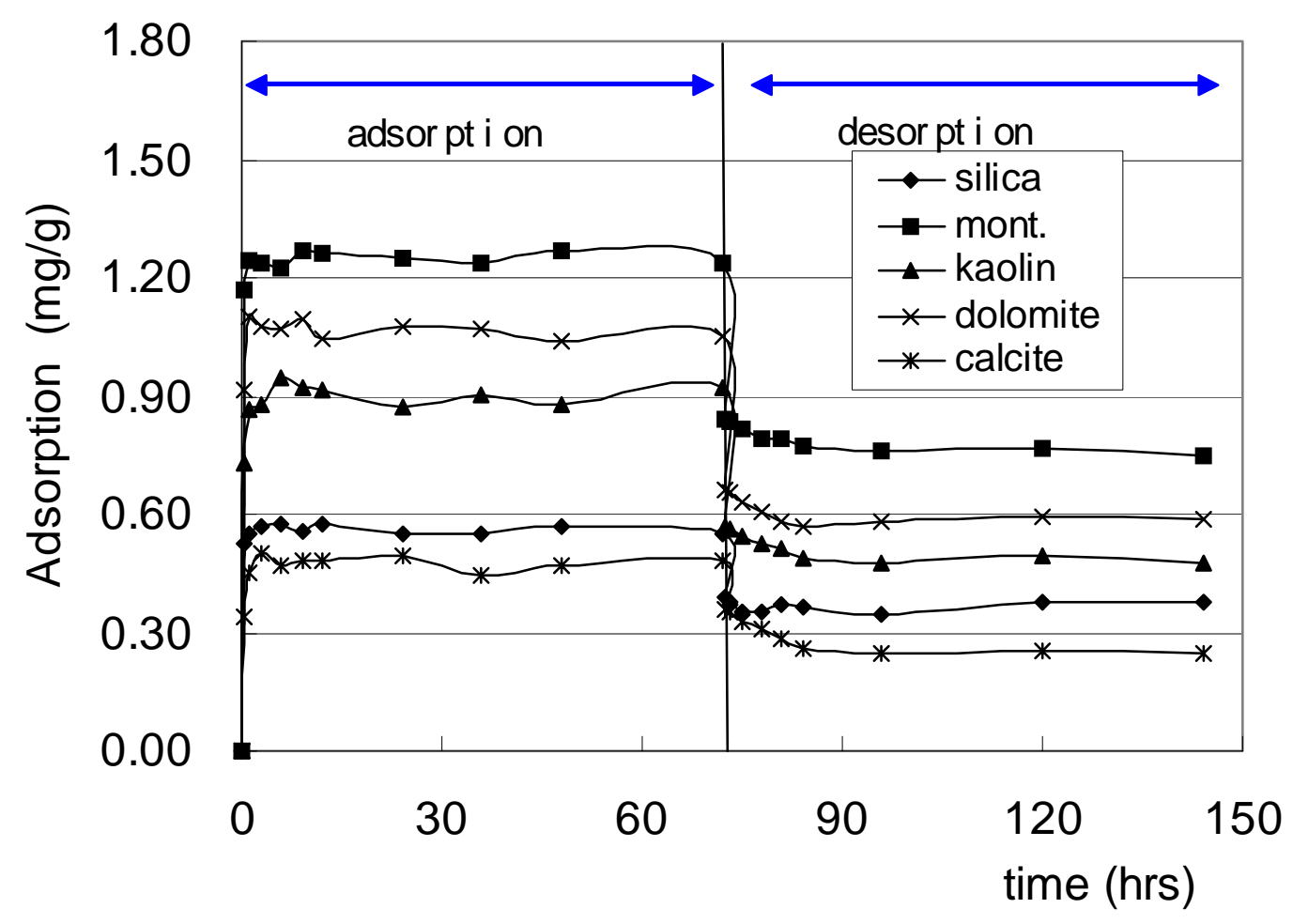

Fig. 3-7. Comparison of CD adsorption and desorption onto five minerals.

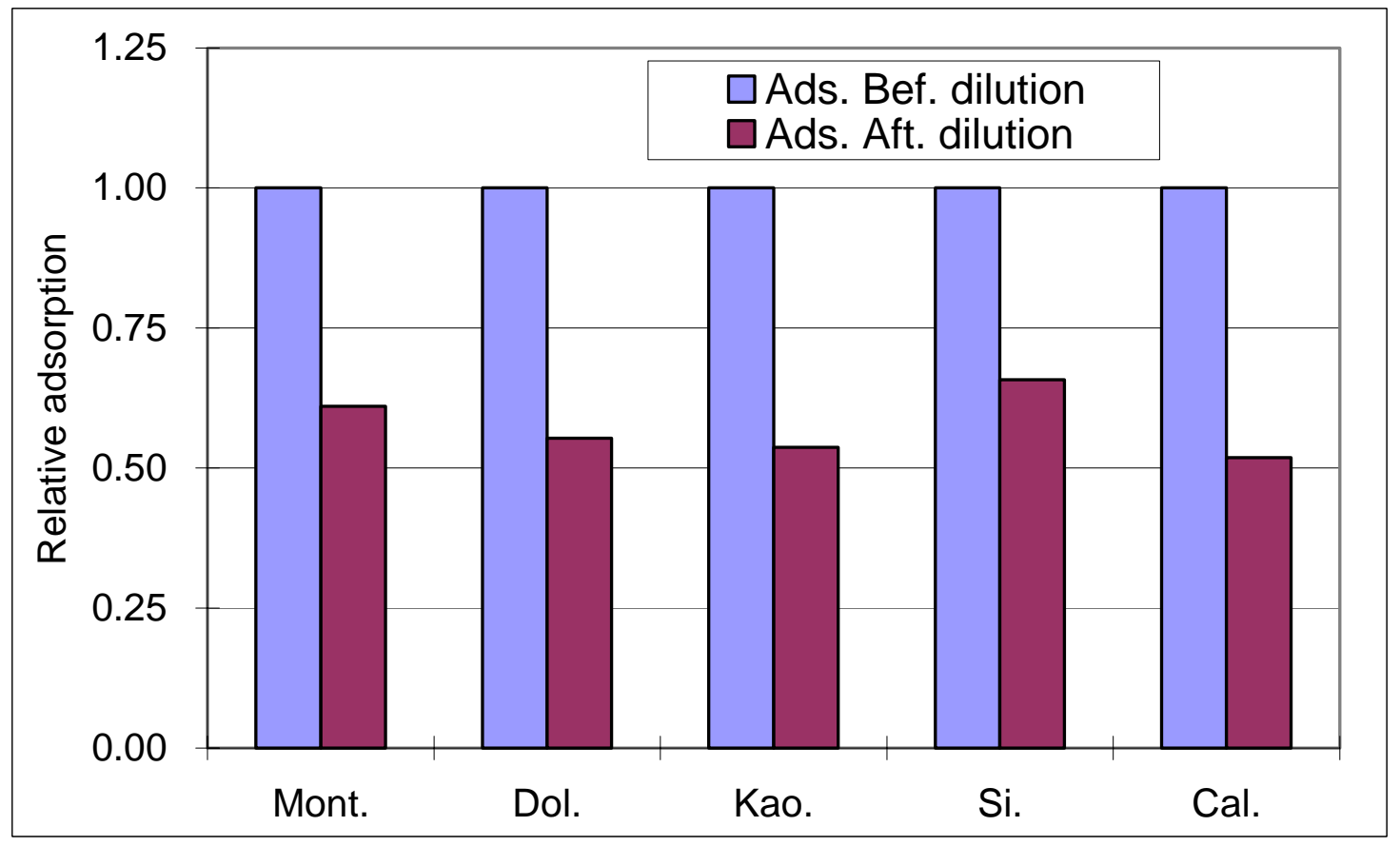

Fig. 3-8. Comparison of $\mathrm{CD}$ adsorption density onto five adsorbents. 


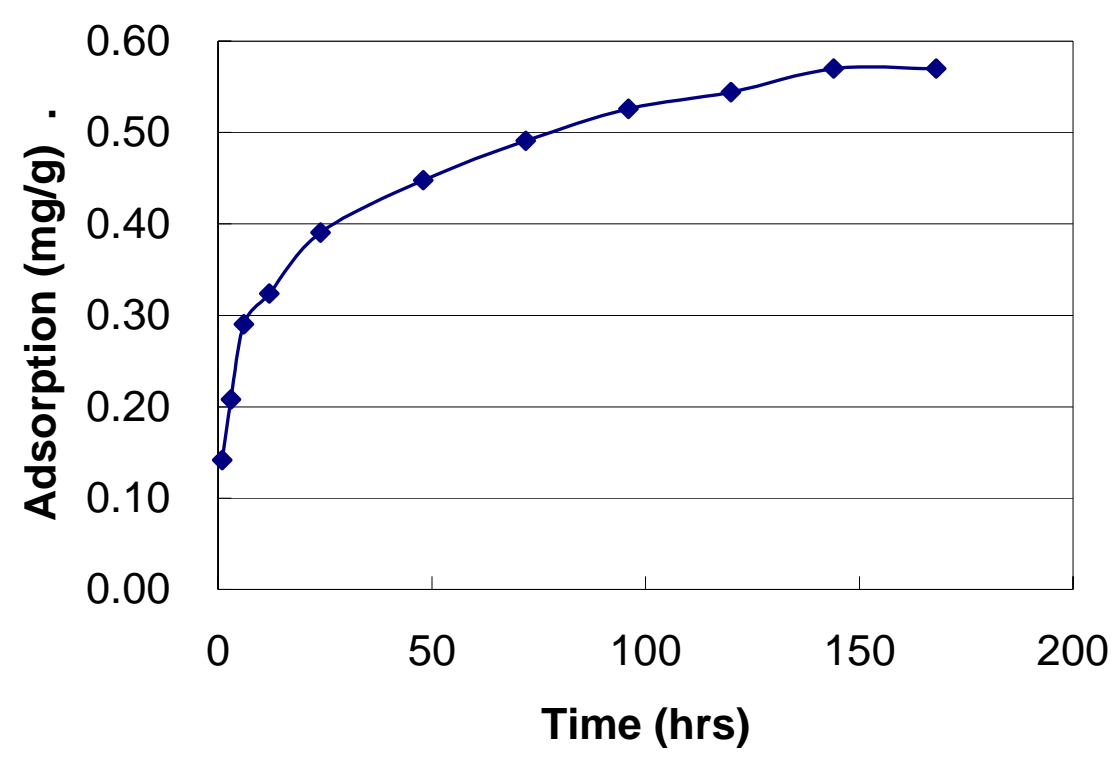

Fig. 3-9. A normal plot of CD adsorption density with time (Berea).

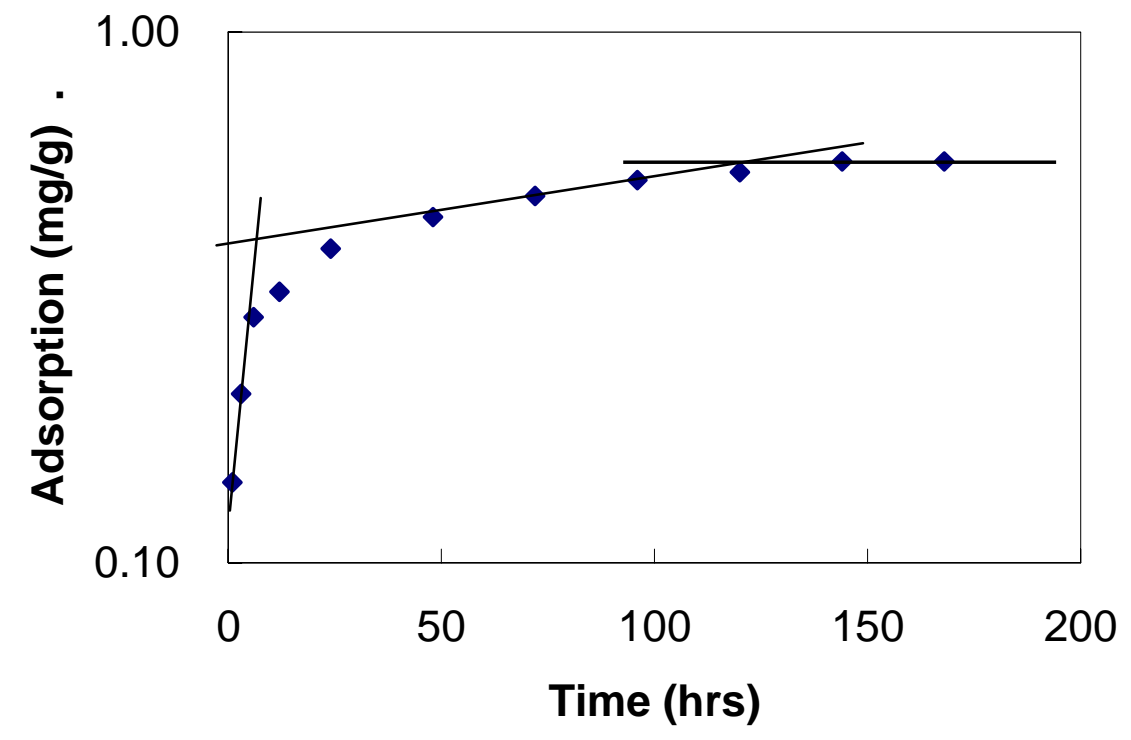

Fig. 3-10. A semilog plot of CD adsorption density with time (Berea). 


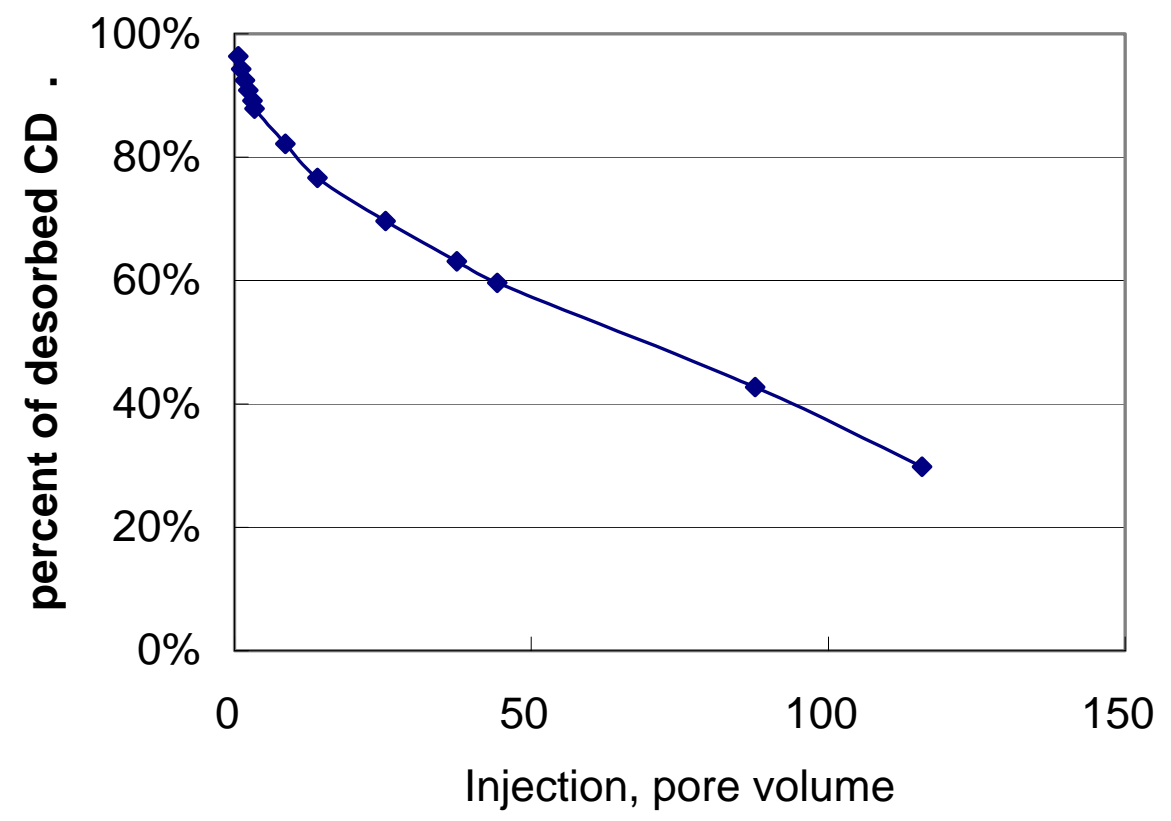

Fig. 3-11. Percent of CD desorbed from Berea sandstone vs. pore volume.

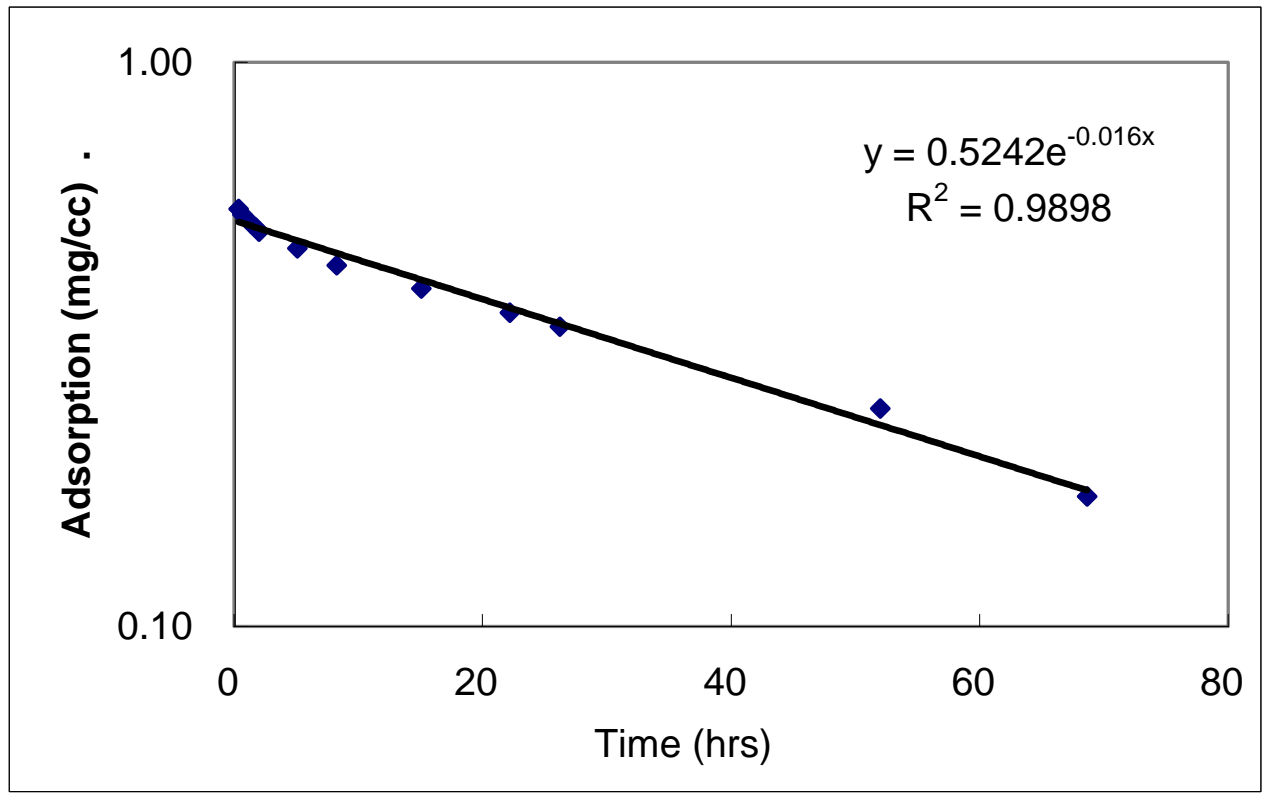

Fig. 3-12. CD desorption from Berea sandstone as a function of time. 


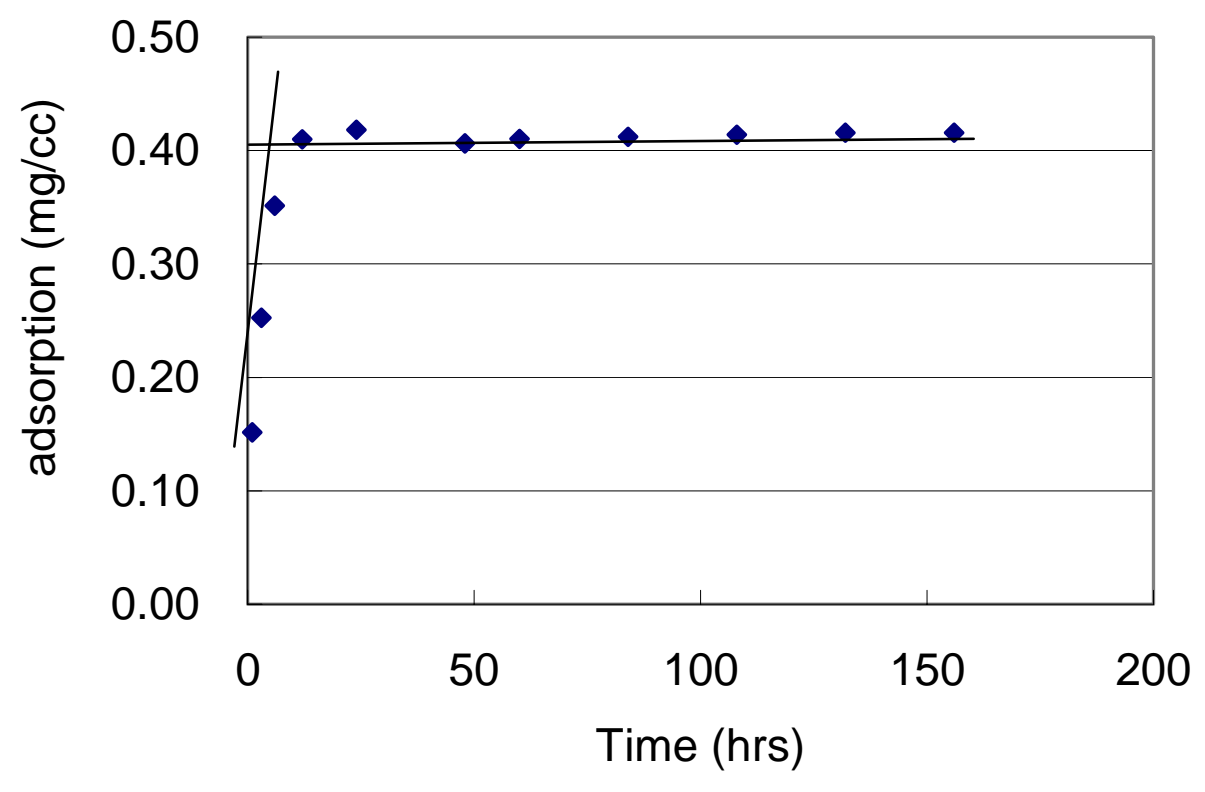

Fig. 3-13. A normal plot of $\mathrm{CD}$ adsorption density with time (limestone).

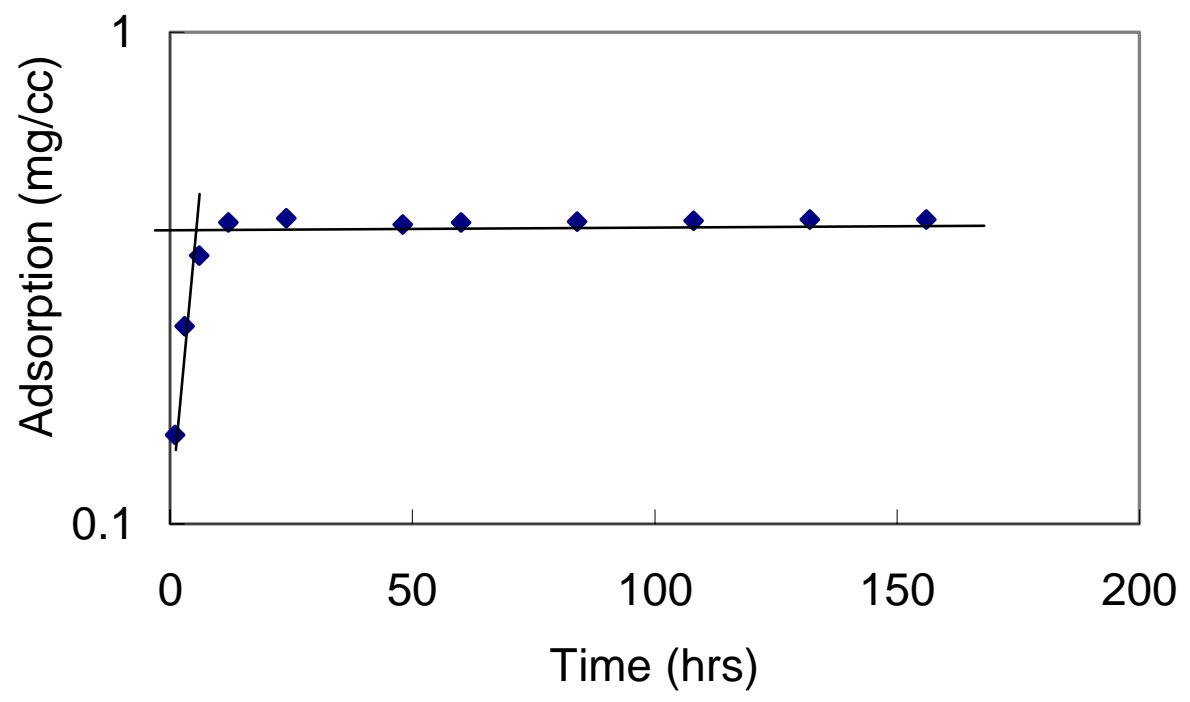

Fig. 3-14. A semilog plot of CD 1045 adsorption onto limestone. 


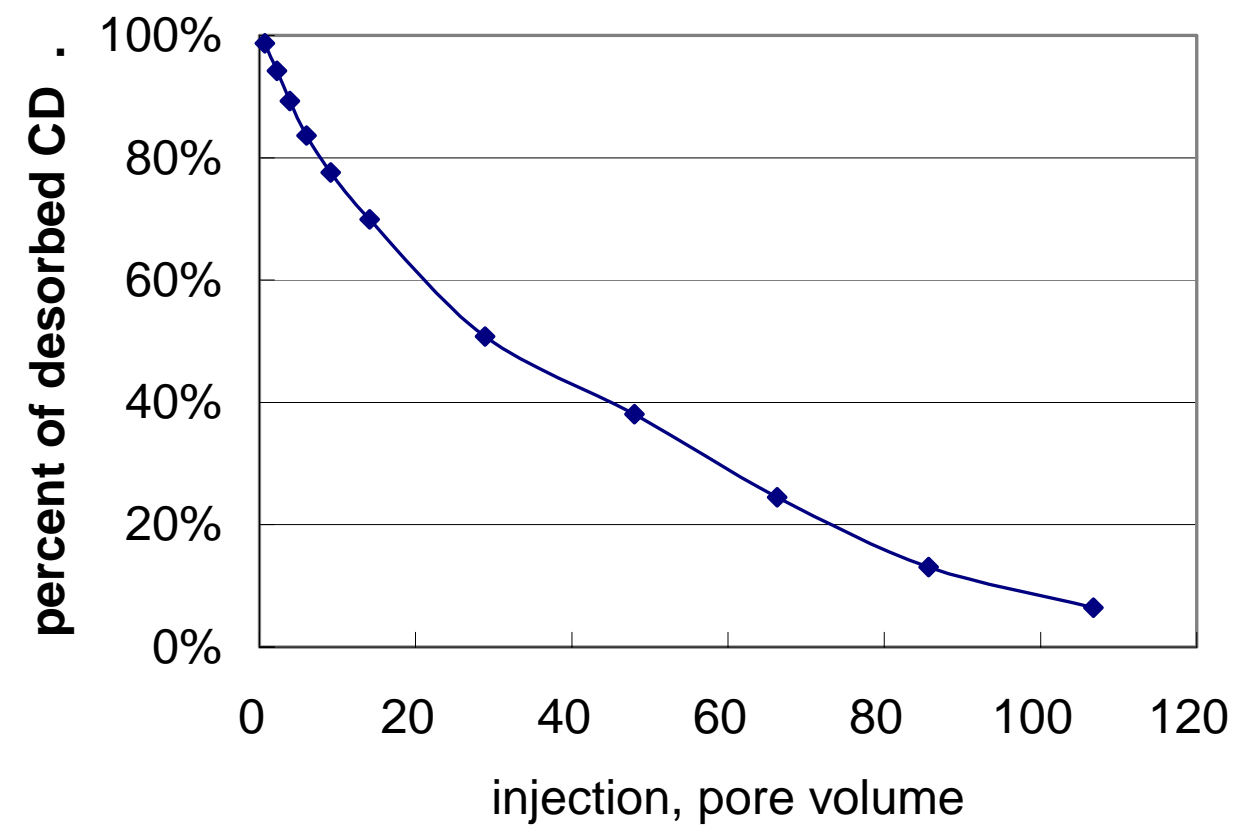

Fig. 3-15. CD desorption from limestone vs. injection pore volume.

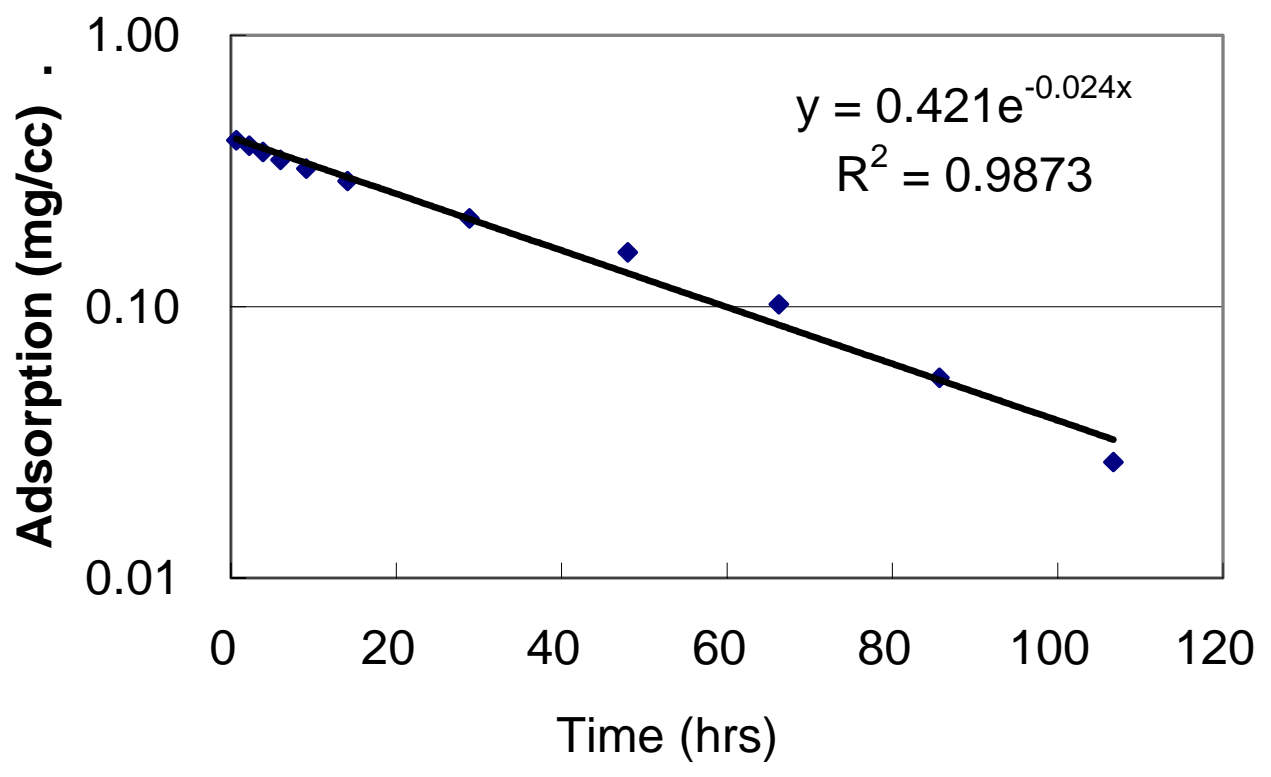

Fig. 3-16. CD desorption from limestone as a function of time (direct flow). 


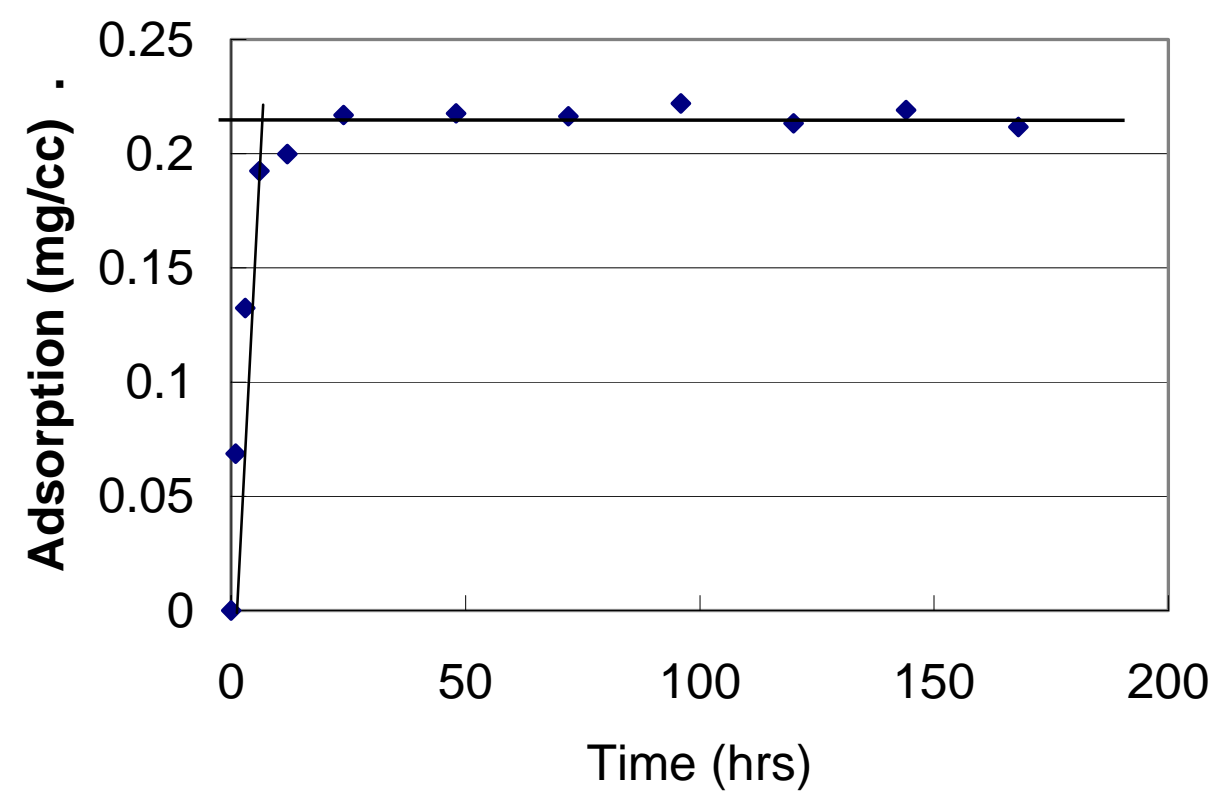

Fig. 3-17. A normal plot of CD adsorption onto dolomite as a function of time.

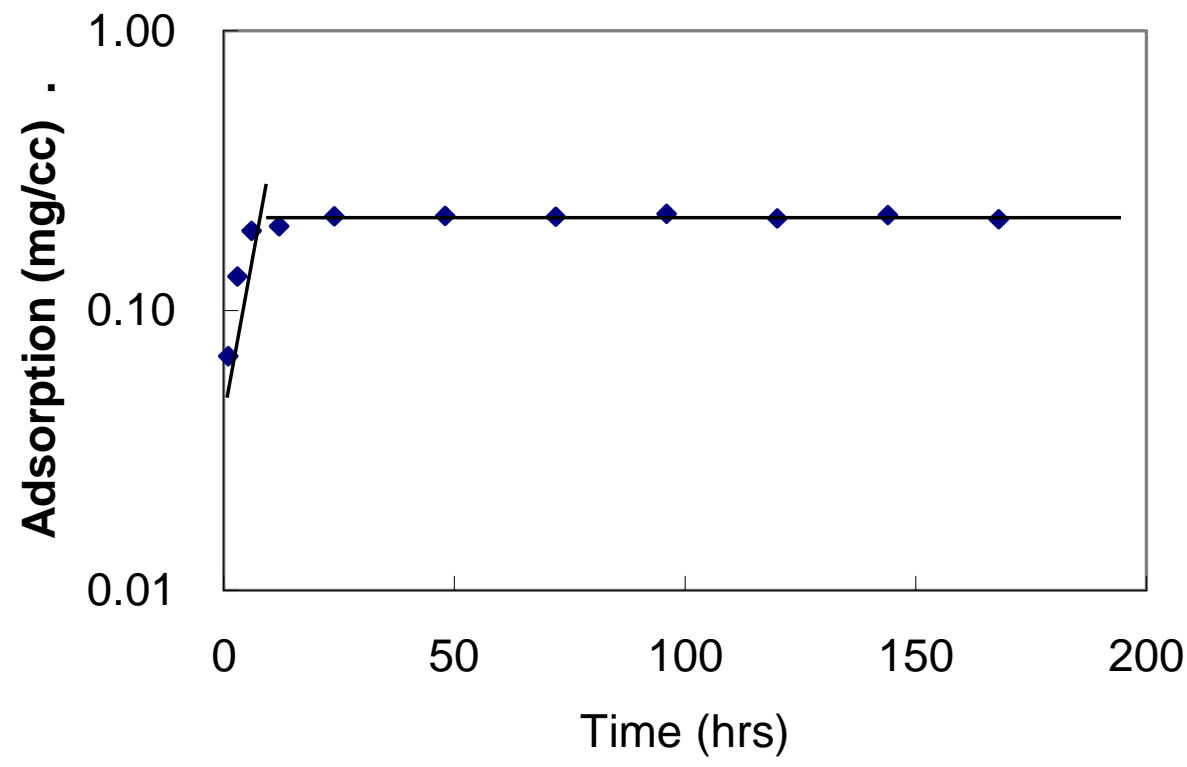

Fig. 3-18. A semiplot of CD adsorption onto dolomite as a function of time. 


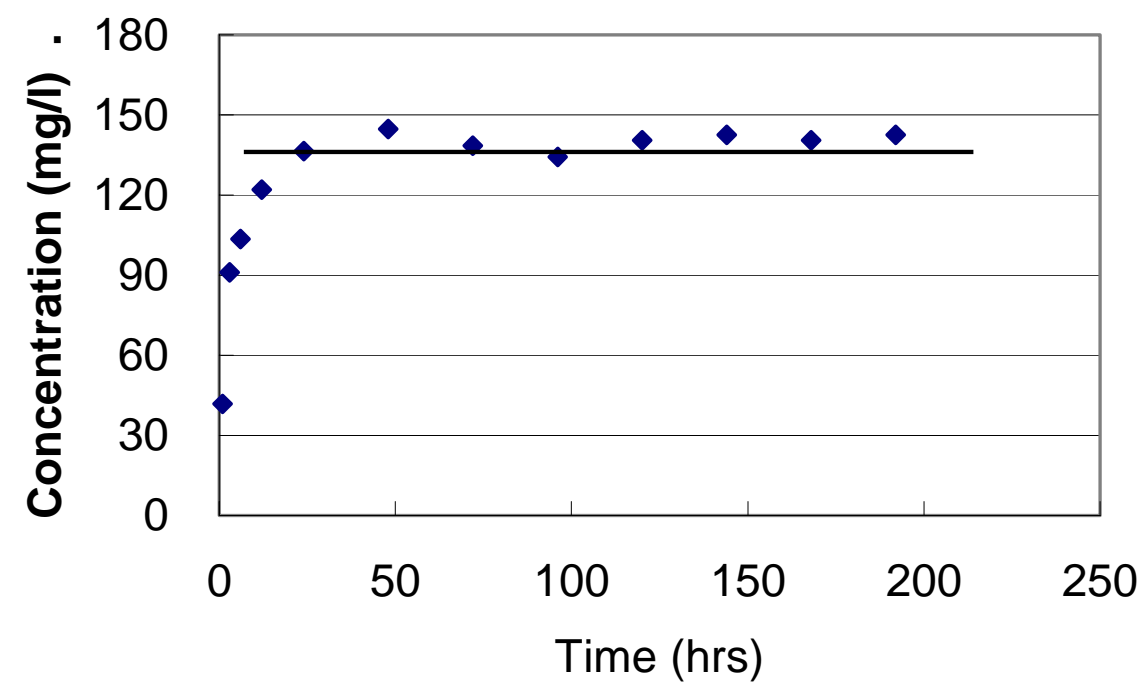

Fig. 3-19. CD concentration in solution as a function of time during its desorption from dolomite.

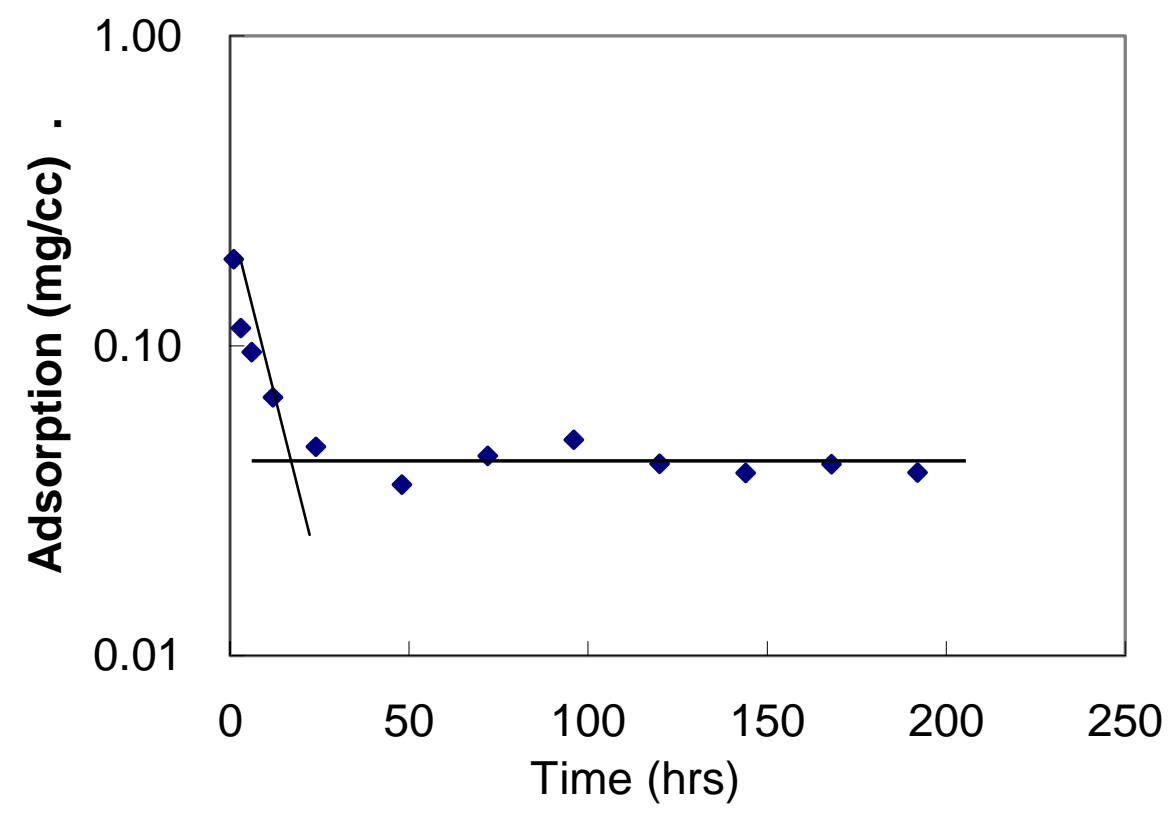

Fig. 3-20. A semi-log plot of CD desorption from dolomite as a function of time. 


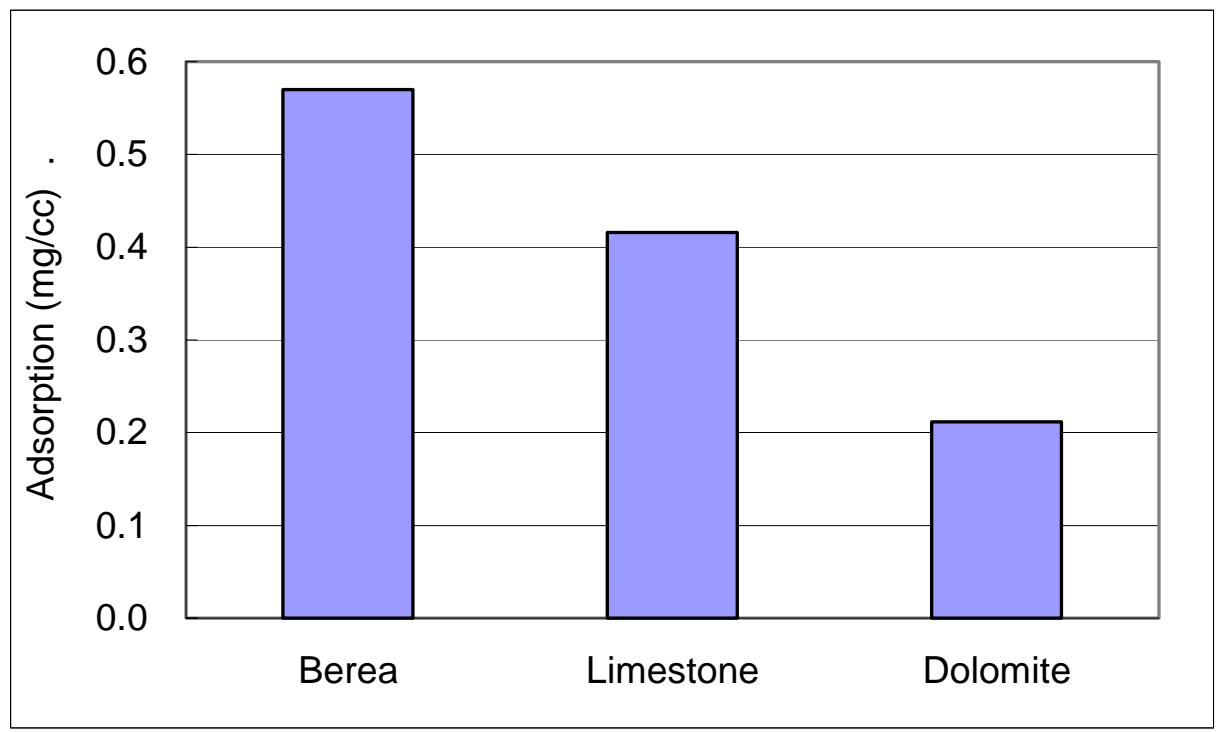

Fig. 3-21. Comparison of CD adsorption onto three porous media.

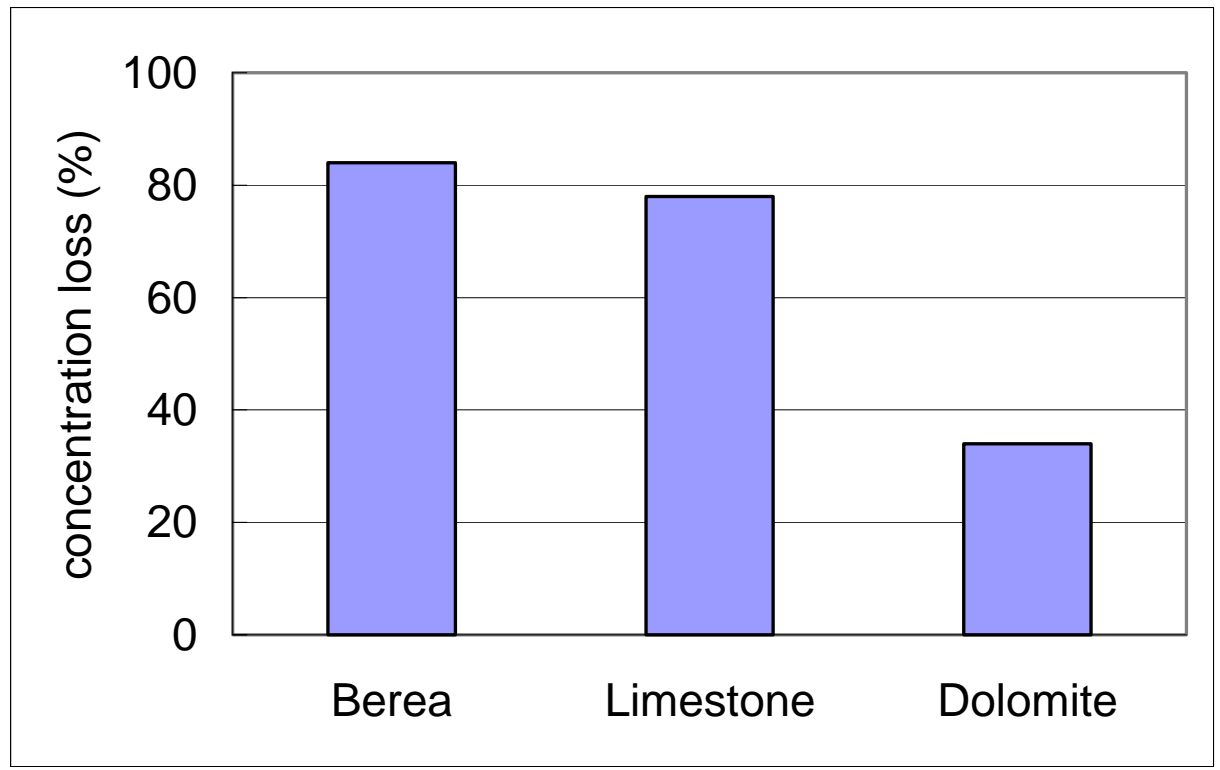

Fig. 3-22. Surfactant loss due to adsorption onto rocks. 


\title{
CHAPTER 4. COMPETITIVE ADSORPTION OF A HYBRID SURFACTANT SYSTEM ONTO FIVE MINERALS, BEREA SANDSTONE, AND LIMESTONE
}

\author{
Abstract \\ This chapter presents the adsorption/desorption results for a mixture of two surfactant systems \\ onto five minerals and two porous media. The systems are composed of Chaser International \\ CD1045 ${ }^{\mathrm{TM}}$ (CD) and a calcium lignosulfonate (CLS), Lignosite ${ }^{\circledR} 100$. Two series of experiments \\ were carried out: (1) static adsorption of CLS, CD, and CLS/CD mixtures onto five pure \\ minerals (silica, montmorillonite, kaolinite, dolomite, and calcite); (2) dynamic adsorption of \\ CLS, CD, and CLS/CD mixtures onto core samples of Berea sandstone and Indian limestone. All \\ experiments were performed at $40^{\circ} \mathrm{C}$ using a $2.0 \%$ brine solution with concentrations of 500 \\ $\mathrm{mg} / \mathrm{l}$ of $\mathrm{CD}$ and 5,000 $\mathrm{mg} / \mathrm{l}$ of CLS.
}

Static adsorption experiment results showed that: (1) CLS adsorption density onto silica is less than background noise (zero); on the other four minerals, adsorption density ranged from 0.5 to $10 \mathrm{mg}$ CLS per g of mineral; (2) CD adsorption density onto the five minerals ranged from 0.4 to $1.2 \mathrm{mg}$ CD per g of mineral; (3) CD adsorption density onto the five minerals decreased by 20 to $70 \%$ when mixed with CLS.

Dynamic adsorption experiment results showed that: (1) The times required to reach adsorption equilibrium for both CD and CLS were longer for Berea sandstone than for Indiana limestone and for both porous media, were much longer than those for the non-porous minerals; (2) Competitive adsorption generally took several days to reach equilibrium.

Stability and interfacial tension tests on both injected and effluent samples were performed. Results correlated well with the adsorption/desorption tests; for example, foaming capability was lost in some systems due to adsorption.

\section{Introduction}

In order to minimize good foaming surfactant adsorption, the system of a sacrificial agent, calcium lignosulfonate (CLS), and a good foaming agent, Chaser CD1045 ${ }^{\mathrm{TM}}$ (CD), was evaluated in earlier publications. ${ }^{1-10}$ This combination showed synergistic improvement, with 
adsorption experiment results showing that lignosulfonate could reduce surfactant loss and surfactant concentration for a foam that was rock type- and injection scheme-dependent.

This chapter reports results of a study that expands on our previous work by examining desorption with adsorption characteristics onto two rock types and in addition examining five pure minerals. This study includes:

1. CLS adsorption/desorption onto two rock types: sandstone and carbonate,

2. CLS adsorption/desorption onto five minerals common in reservoir rock,

3. CD adsorption/desorption onto two rock types: sandstone and carbonate,

4. CD adsorption/desorption onto several minerals common in reservoir rock, and

5. Competitive adsorption of CLS and CD onto two different rock types.

The objectives of this study were:

1. To identify CLS and CD propagation mechanisms through different porous media;

2. To understand factors controlling the adsorption/desorption of the co-surfactant system;

3. To optimize adsorption/desorption of surfactant and sacrificial agent concentration of the system.

This research will contribute to the development of a systematic approach for selecting and formulating surfactant systems with minimal adsorption levels.

\section{Experimental Procedures}

Chemicals. The CLS used in this study was Lignosite ${ }^{\circledR} 100$, which was obtained from the Georgia-Pacific Corporation. The product provided is a powder produced by sulfonation of softwood lignin. CD, the good foaming agent, is Chaser CD1045 ${ }^{\mathrm{TM}}$, which was identified as one of the best foaming agents in several earlier studies ${ }^{8-11}$ and was supplied by Chaser International as $46.7 \mathrm{wt} \%$ active aqueous solution. Dimidium Bromide-Disulphine Blue Indicator, used for anionic surfactant determination, supplied by BDH Laboratory Supplies, was used to detect CD as described in an earlier publication. ${ }^{2}$ HPLC grade chloroform containing approximately $0.75 \%$ ethanol as a preservative was used as part of the CD process. $2 \%(1.5 \mathrm{wt} \% \mathrm{NaCl}$ and $0.5 \mathrm{wt} \%$ $\mathrm{CaCl}_{2}$ ) synthetic brine was prepared and used in all solutions. 
Adsorbents. Five minerals common in oil reservoirs were used as adsorbents: silica, kaolinite, montmorillonite, calcite, and dolomite. These five minerals were described in details in Chapter 2. Two types of porous media were used: Berea sandstone and Indian limestone quarried cores. There were no visual fractures in these cores.

Analytical Method to Detect Surfactant Concentration. CLS is a colored solution, so a spectrophotometer was used directly to determine concentration. Concentration versus wavelength adsorption was linear at CLS concentrations below $400 \mathrm{mg} / \mathrm{l}$ at the maximum adsorption wavelength of $283 \mathrm{~nm}$. CD is a multi-component formulation and is colorless in solution. A colorimetric method was suggested by the manufacturer and used in this study. Since $\mathrm{CD}$ is colorless, an indicator solution of Dimidium Bromide-Disulphine blue was used. The absorbance of CD solution had an excellent absorbance peak at $523 \mathrm{~nm}$, which created minimal interference with the CLS absorption at $283 \mathrm{~nm}$ when mixed with CD in some of the tests. As with CLS, the linear region occurred at concentrations below $400 \mathrm{mg} / \mathrm{l}$. More details of the procedure to determine unknown concentrations of CLS and CD may be found in Chapters 2 and 3 and earlier publications. ${ }^{2,7}$

Mixture Interference on Concentration Determinations. Figures 4-1 and 4-2 show the influence of CD on CLS absorbance at $283 \mathrm{~nm}$ and CLS on CD absorbance at $523 \mathrm{~nm}$, respectively. CD has little effect on CLS at $283 \mathrm{~nm}$ in the range of 50-200 $\mathrm{mg} / 1$ of CD. In this study the concentration of CLS was generally about ten times that of CD, and thus when diluted for CLS determination CD was always less than $100 \mathrm{mg} / \mathrm{l}$.

Absorbance of CD with different concentrations of CLS at $520 \mathrm{~nm}$ was measured to determine the effect of CLS concentration on absorbance measurement results for CD in the hybrid CDCLS system. All samples were measured at room temperature. Figure 4-2 presents the absorbance measurement results of CD with different concentrations of CLS at $520 \mathrm{~nm}$. The influence of the presence of CLS on CD absorption at $523 \mathrm{~nm}$ increases with CLS concentration. For each series of CD concentrations with a constant concentration of CLS, the plot of CD concentration versus absorbance of wavelength $520 \mathrm{~nm}$ is linear (see Fig. 4-2), with the corresponding $\mathrm{R}^{2}$ better than 0.99 for each system. Their equations and corresponding $\mathrm{R}^{2}$ were 
shown in Table 4-1. These results were used in determining unknown CD concentration in mixed systems. The determining principles are shown as follows.

According to the fit equations listed in Table 4-1, Figures 4-3 and 4-4 was plotted. Figure 4-3 shows the relationship of fit line slope and CLS concentration. It can be seen that they have a good relationship and the fit equation is

$$
y=0.3096 x+2062.6\left(R^{2}=0.9951\right)
$$

Figure 4-4 shows that the relationship of the fit line intercept and CLS concentration, and it is also linear. The fit equation is:

$$
\mathrm{y}=0.0078 \mathrm{x}+53.4467 \quad\left(\mathrm{R}^{2}=0.9690\right)
$$

Because CD1045 has little effect on the CLS absorbance shown in Fig. 4-1, the CLS standard calibration curve without CD1045 was directly used to calculate CLS concentration. For CD1045 concentration calculation, new calibration equations were derived using Eqs. 4-1 and 42. For example, if the CLS concentration is $2000 \mathrm{mg} / \mathrm{l}$, the corresponding calibration equation can be determined using the following steps:

(1) Use Eq. $4-1$ to calculate slope: $y=0.3096 * 2000+2062.6=2681.8$

(2) Use Eq. 2 to calculate intercept: $y=0.0078 * 2000+53.4467=69.0467$, therefore,

(3) The standard calibration equation is: Conc. $=2681.8 *$ Abs. +69.0467

\section{Adsorption/Desorption Setup and Method}

Dynamic Methods. Two dynamic methods similar to those described in Chapter 2, circulation and flow-through experiments, were used to study surfactant adsorption/desorption onto porous media. The amount of surfactant adsorbed is expressed as the mass of CLS adsorbed per bulk volume of rock $\left(\mathrm{mg} / \mathrm{cm}^{3}\right)$.

Static Adsorption Method. Static experiments were performed to study surfactant adsorption onto five non-porous minerals. A specific volume of solution with a desired surfactant concentration was pipetted into a bottle. The bottle and the selected mineral were kept in a thermostatic bath to allow the solution to achieve thermal equilibrium. Then a weighed amount of mineral was poured into the bottle and vigorously agitated by hand for about a minute. This was then placed into a mechanical shaker and agitated continuously for $24 \mathrm{hrs}$ and then left 
undisturbed for another $48 \mathrm{hrs}$. A sample was removed and centrifuged at $2000 \mathrm{rpm}$ for $20 \mathrm{~min}$, and then the supernatant solutions were separated by decantation from the vial of the solids after gravity sedimentation. The concentration difference between the stock and the sample were used to evaluate the adsorption density.

Foam IFT and Stability Apparatus. The drop weight method of measuring the interfacial tension between two fluids consists of visually determining in a sapphire tube the number of drops falling or rising from a capillary tube while injecting a known volume of fluid at a measured temperature and pressure (Fig. 4-5). Stability is determined using the same sapphire visual cell (Fig. 4-5). In this study, the cell was first filled with the aqueous solution to be tested. The aqueous system was brought to the desired pressure by means of a positive displacement pump. The pressure difference between the $\mathrm{CO}_{2}$ tank and the oil/surfactant-solution tank was determined and brought to zero. At this point $\mathrm{CO}_{2}$ was allowed to flow from the capillary tube into the surfactant solution. The dense $\mathrm{CO}_{2}$ flowed upward through a needle at the lower end of the cell. Depending on the effectiveness of surfactants, the bubbles either formed a layer of foam-like dispersion at the top of the sapphire tube or coalesced into a clear layer of dense $\mathrm{CO}_{2}$. After a standard volume of $\mathrm{CO}_{2}$ was introduced, the pump was stopped and the stability of foam determined by measuring the foam layer thickness for 90 minutes. More details on a similar system can be found in earlier publications. ${ }^{12}$

\section{Results and Discussion}

Static adsorption onto five minerals. Static adsorption experiments were run to analyze the adsorption of CD, CLS and their mixture onto five minerals. For the system of CD and montmorillonite, the ratio of solid and liquid is 1:4 (3 g solid was put into $12 \mathrm{~g}$ solution); for all the other systems, the ratio of solid and liquid is $1: 3$. All experiments were performed at $40^{\circ} \mathrm{C}$, at ambient pressure, and using $2 \%$ brine solution. All surfactant concentrations were $500 \mathrm{mg} / \mathrm{l} \mathrm{CD}$ and 5,000mg/l CLS. The mixture was composed of $500 \mathrm{mg} / \mathrm{l} \mathrm{CD}$ and 5,000 mg/l CLS.

Figure 4-6 compares CD adsorption density onto five minerals for CD and mixture solutions. In each system, adsorption for the $\mathrm{CD}$ solution was greater than $\mathrm{CD}$ adsorption in the mixture. Therefore, adding CLS reduced CD adsorption on each adsorbent studied. Adsorption for CD solution in decreasing order was: montmorillonite > dolomite > kaolinite > silica > calcite; 
compared to the mixture where the order of CD adsorption was dolomite $>$ kaolinite $\sim$ silica $>$ montmorillonite > calcite. Figure 4-7 compares the percentage of decrease in adsorption in the mixture compared to CD alone. CLS reduces CD adsorption $20 \%$ to $70 \%$ for these five minerals. The relative reduction in CD adsorption due to the presence of CLS was significantly greater for the clays (montmorillonite and kaolinite) than for the other minerals, which may be related to their different mineral structures.

Figure 4-8 compares CLS adsorption density onto five minerals for pure CLS and the mixture solution. CLS adsorption onto silica was essentially zero, and the decreasing order of CLS onto the five minerals was the same for both systems: montmorillonite > kaolinite > dolomite > calcite > silica. CD has no obvious influence on CLS adsorption onto silica but reduces CLS adsorption onto the other four minerals. Figure 4-9 compares the results of CLS reduction onto four minerals when mixed with CD. CD reduced CLS adsorption from $10 \%$ to $48 \%$ for these systems. Opposite to the effect of CLS on CD, the effect of CD on CLS adsorption is less onto clay than onto the other minerals.

Dynamic sorption onto porous media. Circulation experiments were carried out to study the adsorption and desorption of CLS, CD, and a mixture onto Berea sandstone and Indiana limestone. Each circulation process took more than seven days at a circulation rate of 0.5 $\mathrm{cm}^{3} / \mathrm{min}$. Table 4-2 shows parameters of the two cores. Table 4-3 lists the experiment schedule in each core. Except between Schedule 2 and 3 in Table 4-3, about $400 \mathrm{ml}$ of tetrahydrofuran (THF) was injected through the core between tests to clean out all remaining surfactants. Again, the initial CD and CLS solution concentrations were $500 \mathrm{mg} / \mathrm{l}$ and 5,000mg/l, respectively, with the mixture containing $500 \mathrm{mg} / \mathrm{l} \mathrm{CD}$ and 5,000 $\mathrm{mg} / \mathrm{l} \mathrm{CLS}$.

Berea Sandstone. The results of three different CD injection schemes are shown in Figs. 4-10 and 4-11. Figure 4-10 gives the CD concentration change with time and Fig. 4-11 indicates the $\mathrm{CD}$ adsorption density increase versus times. The designation "Pure CD" refers to the injection of CD solution alone (Schedule 1 in Table 4-3). The designation "Preadsorb CLS" refers to the injection scenario of CD solution circulation (Schedule 3 in Table 4-3), following the injection of 
a CLS solution (Schedule 2 in Table 4-3). The designation of "Mixture" indicates the injection of the CD/CLS mixture with no presoak (Schedule 4 in Table 4-3).

The adsorption concentration of $\mathrm{CD}$ was initially difficult to distinguish for the three systems (Fig. 4-11). With time, the three deviate, with the order of increasing adsorption being "Mixture" $<$ "Preadsorb CLS" < "Pure CD". Comparing the final CD concentration for the three schemes, CLS increases the equilibrium concentration of $\mathrm{CD}$ in solution by interfering with the adsorption of $\mathrm{CD}$, thus increasing the probability of having sufficient surfactant concentration in solution to form foam (third photo in Fig. 4-29).

CLS Adsorption versus Time. Figures 4-12 and 4-13 compares the results of injecting "Pure CLS" into Berea sandstone (Schedule 2 in Table 4-3) to the injection of the CLS/CD "Mixture" into a Berea sandstone (Schedule 4 in Table 4-3). The adsorption of CLS onto Berea sandstone was still increasing after six days of circulation (Fig. 4-13). Adding CD reduced CLS adsorption onto sandstone by almost $50 \%$.

Competitive Adsorption/Desorption versus Time. To analyze the competitive adsorption of CD and CLS, their adsorption change with time during the circulation of the mixture was plotted in Fig. 4-14 using Schedule 4 in Table 4-3. The relative adsorption or ratio of CD to CLS is also shown in this figure. The relative adsorption increased greatly during the first few hours, after which both increased slowly at about a constant ratio.

After the circulation adsorption experiment was run for $216 \mathrm{hrs,} \mathrm{the} \mathrm{solution} \mathrm{used} \mathrm{for} \mathrm{circulation}$ was displaced by a surfactant-free $2 \%$ brine to observe the desorption process using the circulation. Figure 4-15 compares the CLS and CD concentration change in the circulation fluid versus time for two desorption experiments. In the second desorption the equilibrated solution is removed from the solution bottle and again a surfactant-free $2 \%$ brine is added. During the desorption process, CD and CLS concentration in the circulation fluid increased rapidly during the first few hours, because much of the initial surfactant concentration in the circulating fluid came from the CD and CLS solution left in the circulation system lines and free fluid in the pores of the core. After the CD and CLS in the bottle mixed well with the solution in the circulation 
line and pore spaces of the core, a further increase in concentration occurred from equilibration of fluid with the fluid in micropores, dead end pores, and surfactant absorbed on the surface.

Figure 4-16 compares CD adsorption and CLS desorption profiles during Schedule 3 in Table 43 for sandstone. Again CLS had been circulated to reach adsorption equilibrium and then during this step the CLS solution in the circulation bottle was changed to the CD solution. During this test CD adsorbed onto the sandstone and CLS desorbed off the sandstone. This trend decreased with time as the system approached equilibrium. CD did not completely replace CLS adsorbed onto the sandstone, thus reducing the total CD adsorbed compared to having no CLS present.

After the above $\mathrm{CD}$ adsorption process was completed, a desorption experiment (circulation) was performed by replacing the surfactant solution in the circulation bottle with surfactant-free $2 \%$ brine in the bottle, desorption phase of Schedule 3 in Table 4-3. Figure 4-17 shows the CD and CLS concentration change in the circulation bottle versus time. The CLS desorption curve has a similar shape but lower magnitude to that shown for CLS desorption during CD adsorption shown in Fig. 4-16. Comparing CD and CLS concentration curves versus time during desorption from the $2^{\text {nd }}$ desorption process after the CD/CLS mixed solution injection, Fig. 4-15, with desorption after the preadsorb CLS injection/CD injection, Fig. 4-17, shows a similar trend. A rapid increase in concentration during fluid dilution, followed by a slower change from desorption.

Comparison of CD and CLS Adsorption Using Different Injection Schemes. Figure 4-18 compares the adsorption results for three injection schemes. Figure 4-19 shows the relative adsorption for the three injection schemes assuming that CD or CLS adsorption is 100\% when pure CD or CLS is injected. The adsorption reduction in the mixed systems compared to pure component injection was about $30 \%$ and $45 \%$ for CD and CLS, respectively. The reduction in CD adsorption for the CLS preadsorbed- and mixture-injection schemes for CD were comparable. Because CLS desorbed from core and the free CLS solution in pore space of core can continue to propagate through reservoir, which aids in lowering CLS mass used as a sacrificial agent. 


\section{Indiana Limestone}

CD Adsorption versus Time. Figure 4-20 shows CD concentration change with time for three different injection schemes and Fig. 4-21 compares their corresponding adsorption changes with time. The adsorption equilibrium time for $\mathrm{CD}$ adsorption onto limestone was much faster than that of $\mathrm{CD}$ adsorption onto Berea sandstone, though it was slower than that of the static nonporous adsorption tests. However, adding CLS prolonged the equilibrium time whether preadsorb (Schedule 2/3 in Table 4-3) or mixture injection (Schedule 4 in Table 4-3) was used. Also, CLS reduced CD adsorption onto limestone by about 30\% (see Figs. 4-25 and 26).

CLS Adsorption versus Time. CLS concentration and adsorption changes with time were compared in Figs.4-22 and 4-23 when pure CLS solution and the mixture were circulated through the limestone. It can be seen that the equilibrium adsorption time of CLS for pure CLS solution is longer than that for the mixture. CD reduced CLS adsorption onto the limestone by about $25 \%$.

Competitive Adsorption/Desorption versus Time. Figure 4-24 compares CD and CLS adsorption profiles when a mixture was injected (Schedule 4 in Table 4-3). The relative adsorption is also shown in the figure. The relative adsorption increased quickly during the first few hours; followed by a slow decrease.

Comparison of CD and CLS Adsorption for Different Injection Schemes. Figure 4-25 compares the adsorption results of CD and CLS onto limestone and Fig. 4-26 compares their relative adsorption. Both preadsorption of CLS or injection of the mixture reduced CD adsorption by more than $30 \%$, and CD reduced CLS adsorption by about $22 \%$ when it was injected in a mixture. Similar to the effect of the injection scheme of CD adsorption onto Berea sandstone, preadsorbed and mixture injection had similar contributions to CD adsorption reduction. 
Foam Stability of the Effluent from Coreflooding Experiments. Table 4-4 summarizes CD and CLS circulation fluid concentration values after each adsorption scheme was terminated. Adsorption can reduce CD concentration in the flowing fluid from $500 \mathrm{mg} / \mathrm{l}$ to $81 \mathrm{mg} / \mathrm{l}$ or 108 $\mathrm{mg} / \mathrm{l}$ onto sandstone or limestone, respectively (Schedule 1 in Table 4-3). This reduction can cause a good foaming agent to lose the ability to form foam. Adding CLS in solution or presorbing CLS on Berea sandstone or limestone aids in maintaining CD concentration a higher level, even if CD adsorption is not eliminated with the addition of CLS or presoaking on adsorbents.

Foam IFT and Stability. $\mathrm{CO}_{2}$ foam IFT and stability were determined on solutions both before and after adsorption/desorption tests for the porous media. Effluent of the adsorption tests in the core in every case measured had a reduction of surfactant concentration (Table 4-4). Normally, IFT increases with a decrease of surface-active agents (surfactants). This is confirmed in comparing results of Tables 4-5 and 4-6 with Table 4-4 results for solution before and after flooding tests. Table 4-5 is the IFT for each system; this can be compared to a summary of the stability tests in Table 4-6. The IFT increased with the decrease of CD concentration (Table 4-4). Sufficient CD was adsorbed (Table 4-4) that the surfactant solution effluent from the sandstone or limestone core was unstable (Fig. 4-27). CLS was unstable both before and after contact with limestone or sandstone (Table 4-6 and third picture in Figs. 4-27). The CLS concentrations were $5000 \mathrm{mg} / \mathrm{l}$ before and $3693 \mathrm{mg} / \mathrm{l}$ after the limestone core tests.

After preflushing or mixing CLS with the CD brine solution, the loss of CD in the core decreased, resulting in higher concentrations of CD in the effluent. Instead of 81 and 108 in the sandstone and limestone effluent, respectively, it was 209 to 244 in the effluent, respectively. This indicates that in both the systems preflushed with CLS or injecting the mixture with CLS, the $\mathrm{CD}$ adsorbed on the core decreased (Table 4-4). In both cases the effluent had some foaming capability when mixed with CLS but was too weak when preflushed with CLS (Figs. 4-28 and 29). 


\section{RESULTS AND DISCUSSION}

The comparison of the pure minerals to sandstone and limestone indicate that the time to reach equilibrium is much shorter for the mineral in a powder form versus the pore media. This is thought to be due to the ease in which the surfactant comes in contact with the powder in a shaker versus surfaces in a porous media. In all cases for both CD and CLS, adsorption equilibrium occurred in less than one hour at the tested conditions for the pure minerals. In contrast in the porous media equilibrium required several hours for limestone and after 150 hours the sandstone appeared to be reaching a plateau. This is an indication that the adsorption kinetics is relatively fast in the porous media compared to dispersion and diffusion.

Understanding the rate differences between sandstone and limestone is part of an ongoing study in which it is believed that the slower rate of the sandstone is due to diffusion into dead end pores and micropores that are not significant in limestone. In the sandstone it appears that the presence of CLS does not decrease the rate or relative amount of CD adsorption initially (Fig. 415). This is during the initial stage when it is believed that dispersion is the principle mechanism of surfactant delivery to the surface of the core. At the later times diffusion becomes the major transport mechanism and CLS significantly reduces CD adsorption onto the core. This might be due to the higher adsorption of CLS adsorbing onto clay that is a principle constituent in the micropores.

Adsorption onto limestone is complete with in a few hours. The relative effect on adsorption of $\mathrm{CD}$ in the presence of CLS is less. Also, clay is not a significant component of the limestone and the adsorption of CD and CLS on calcite is similar.

IFT and stability of the fluid before and after injection were tested to quantify foaming ability of the solutions. The tests confirmed the reduction of foam stability due to the reduction of surfactant in solution.

\section{Conclusions}

1. Static adsorption experiment results show that:

a. CLS with an initial concentration of $5000 \mathrm{mg} / \mathrm{l}$ adsorption density onto silica is 
essentially zero; CLS adsorption density for the other four minerals ranges from 0.5 to 10 $\mathrm{mg} / \mathrm{g}$ on calcite, dolomite, kaolinite, and montmorillonite, respectively.

b. $\mathrm{CD}$ with an initial concentration of $500 \mathrm{mg} / \mathrm{l}$ adsorption density onto the five minerals ranged from 0.4 to $1.2 \mathrm{mg} / \mathrm{g}$ for calcite, silica, kaolinite, dolomite, and montmorillonite, respectively.

c. CD adsorption density onto five minerals decreased from 20 to $70 \%$ when $\mathrm{CD}$ was mixed with CLS. The reduction of CD adsorption onto clay was higher than that onto the other minerals.

2. Dynamic adsorption experiment results show that:

a. CD adsorption can be greatly reduced by pre-adsorbing CLS onto sandstone or limestone or by injecting a mixture of CD and CLS;

b. Adsorption equilibrium time of CD and CLS onto Berea sandstone is much longer than that onto Indiana limestone;

c. Competitive adsorption of CD and CLS generally took several days.

3. Foam stability and IFT show a decrease in foaming capability similar to that expected due to adsorption.

4. The results from this work are being used to develop optimum solution concentrations of CD and CLS for specific reservoir rock. This will be used to optimize foaming ability, product cost, and adsorption.

\section{References}

1. Syahputra, A.E., "Experimental Evaluation of Lignosulfonate as a Sacrificial Agent in $\mathrm{CO}_{2}$ Foam Flooding,” M.S., New Mexico Tech, Socorro, NM (1999).

2. Tsau, J.-S., Syahputra, A.E., and Grigg, R.B.: "Economic Evaluation of Surfactant Adsorption in $\mathrm{CO}_{2}$ Foam Application," paper SPE 59365 presented at the 2000 SPE/DOE Improved Oil Recovery Symposium, Tulsa, April 3-5.

3. Grigg, R.B., Tsau, J.-S., and Martin, F.D.: "Cost Reduction and Injectivity Improvements for CO2 Foams for Mobility Control," paper SPE 75178 presented at the 2002 SPE/DOE Improved Oil Recovery Symposium, Tulsa, April 13-17 
4. Syahputra, A.E., Tsau, J.-S., and Grigg, R.B.: "Laboratory Evaluation of Using Lignosulfonate and Surfactant Mixture in $\mathrm{CO}_{2}$ Flooding," paper SPE 59368 presented at the 2000 SPE/DOE Improved Oil Recovery Symposium, Tulsa, April 3-5.

5. Tsau, J.S., et al:: "Use of Sacrificial Agents in $\mathrm{CO}_{2}$ Foam Flooding Application," paper SPE 56609 presented at the 1999 SPE Annual Technical Conference and Exhibition, Houston, October 3-6.

6. Tsau, J.-S., Yaghoobi, H., and Grigg, R.B.: "Use of Mixed Surfactants to Improve Mobility Control in $\mathrm{CO}_{2}$ Flooding," paper SPE 39792 presented at the 1998 SPE Permian Basin Oil and Gas Recovery Conference, Midland, March 23-27.

7. Grigg, R.B., "Improved Efficiency of Miscible $\mathrm{CO}_{2}$ Floods and Enhanced Prospects of $\mathrm{CO}_{2}$ Flooding Heterogeneous Reservoirs," Final Report, Contract No. DE-FG26-97BC15047, U.S. DOE, (April 2001).

8. Tsau, J.-S. and Heller, J.P.: "Evaluation of Surfactants for $\mathrm{CO}_{2}$-Foam Mobility Control," paper SPE 24013 presented at the 1992 Permian Basin Oil and Gas Recovery Conference, Midland, March 18-20.

9. Tsau, J.S. et al.: " $\mathrm{CO}_{2}$ Foam Field Verification Pilot Test at EVGSAU: Phase IIIA: Surfactant Performance Characterization and Quality Assurance," paper SPE 27785 presented the 1994 SPE/DOE Ninth Symposium on Improve Oil Recovery, Tulsa, April 1721.

10. Tsau, J.-S. and Grigg, R.B.: "Assessment of Foam Properties and Effectiveness in Mobility Reduction for $\mathrm{CO}_{2}$-foam Floods," paper SPE 37221 presented at the 1997 SPE International Symposium on Oilfield Chemistry, Houston, Feb. 18-21.

11. Kuehne, D.L. et al.: "Evaluation of Surfactants for $\mathrm{CO}_{2}$ Mobility in Dolomite Reservoirs," paper SPE 24177 presented at the 1992 SPE/DOE Symposium on Enhanced Oil Recovery, Tulsa, April 22-24.

12. Lee, H.O., Heller, J.P., and Hoefer, A.M.W.: "Change in Apparent Viscosity of $\mathrm{CO}_{2}$ Foam with Rock Permeability," SPERE (Nov. 1991) 421-428. 
Table 4-1. Standard Curve Equations of CD with Different CLS Concentrations

\begin{tabular}{|c|c|c|c|}
\hline No. & CLS Concentration $(\mathrm{mg} / \mathrm{l})$ & Equation of Standard Curve & $\mathrm{R}^{2}$ Values \\
\hline 1 & 0 & $\mathrm{CONC}=2057.61^{*} \mathrm{ABS}+56.17$ & 0.9991 \\
\hline 2 & 100 & $\mathrm{CONC}=2136.49^{*} \mathrm{ABS}+51.80$ & 0.9987 \\
\hline 3 & 500 & $\mathrm{CONC}=2145.46^{*} \mathrm{ABS}+60.16$ & 0.9996 \\
\hline 4 & 1000 & $\mathrm{CONC}=2406.74^{*} \mathrm{ABS}+57.67$ & 0.9974 \\
\hline 5 & 5000 & $\mathrm{CONC}=3610.11^{*} \mathrm{ABS}+92.99$ & 0.9979 \\
\hline
\end{tabular}

Notes:

ABS refers to the absorbance value measured at $520 \mathrm{~nm}$ from Spectrophotometer (SPECTRONIC ${ }^{\circledR}$ Genesys $^{\mathrm{TM}}$ ) CONC. refers to the concentration of CD1045.

Table 4-2. Parameters of Berea Sandstone and Indiana Limestone

\begin{tabular}{|c|c|c|c|c|c|c|c|}
\hline Core & $\begin{array}{c}\text { Length, } \\
\mathrm{cm}\end{array}$ & $\begin{array}{c}\text { Diameter, } \\
\mathrm{cm}\end{array}$ & $\begin{array}{c}\text { Bulk vol., } \\
\mathrm{cm}^{3}\end{array}$ & $\begin{array}{c}\text { Weight, } \\
\mathrm{g}\end{array}$ & $\begin{array}{c}\text { Pore } \\
\text { Volume }\end{array}$ & $\begin{array}{c}\text { Porosity, } \\
\%\end{array}$ & $\begin{array}{c}\text { Perm., } \\
\text { md }\end{array}$ \\
\hline Sandstone & 6.20 & 3.81 & 70.69 & 142.21 & 11.86 & 16.78 & 224.12 \\
\hline Limestone & 7.90 & 3.81 & 90.07 & 210.23 & 6.64 & 7.32 & 21.89 \\
\hline
\end{tabular}

Table 4-3. Experiment Schedule of CLS, CD and Their Hybrid Adsorption/Desorption onto Berea Sandstones and Limestone

\begin{tabular}{|c|c|c|c|c|c|c|c|}
\hline \multirow{2}{*}{$\begin{array}{l}\text { Procedure } \\
\text { Schedule }\end{array}$} & Preflush & \multicolumn{2}{|c|}{ Adsorption } & \multicolumn{2}{c|}{ Desorption } & \multirow{2}{*}{$\begin{array}{c}\text { Clean } \\
\text { core }\end{array}$} & $\begin{array}{c}\text { Saturated } \\
\text { fluids }\end{array}$ \\
\cline { 3 - 8 } & Chemical & $\begin{array}{c}\mathrm{C}^{*}, \\
\mathrm{mg} / \mathrm{l}\end{array}$ & Method & Brine & & & \\
\hline 1 & $2 \%$ brine & CD & 500 & Open flow & $2 \%$ brine & THF* & $2 \%$ brine \\
\hline 2 & $2 \%$ brine & CLS & 5000 & - & - & - & - \\
\hline 3 & - & CD & 500 & Circulation & $2 \%$ brine & THF & $2 \%$ brine \\
\hline 4 & $2 \%$ brine & CD+CLS & $500 /$ & Circulation & $2 \%$ brine & THF & \\
& & & 5000 & & & & \\
\hline
\end{tabular}

Notes: "C" refers to chemical concentration.

"THF": $400 \mathrm{ml}$ of THF was injected.

Table 4-4. Equilibrium Concentration for Sandstone and Limestone with Starting Concentration of $500 \mathrm{mg} / \mathrm{l}$ of CD and $5000 \mathrm{mg} / \mathrm{l}$ of CLS

\begin{tabular}{|c|c|c|c|c|}
\hline Scheme & \multicolumn{2}{|c|}{ Sandstone } & \multicolumn{2}{c|}{ Limestone } \\
\hline & CD & CLS & CD & CLS \\
\hline CD or CLS & 81 & 3730 & 108 & 3693 \\
\hline CD/CLS Mixture & 209 & 4289 & 233 & 4024 \\
\hline Preadsorb CLS, then CD & 212 & 1389 & 244 & No data \\
\hline
\end{tabular}


Table 4-5. IFT values before and after Adsorption for Sandstone and Limestone with Starting Concentration of $500 \mathrm{mg} / \mathrm{l}$ of CD and $5000 \mathrm{mg} / \mathrm{l}$ of CLS

\begin{tabular}{|c|c|c|c|c|c|c|}
\hline IFT, $\mathrm{mN} / \mathrm{m}$ & \multirow{2}{*}{\multicolumn{2}{|c|}{$\begin{array}{l}\text { Before } \\
\text { Injection }\end{array}$}} & \multicolumn{4}{|c|}{ Effluent Stream } \\
\hline \multirow[t]{2}{*}{ Scheme } & & & \multicolumn{2}{|c|}{ Sandstone } & \multicolumn{2}{|c|}{ Limestone } \\
\hline & $\mathrm{CD}$ & CLS & $\mathrm{CD}$ & CLS & $\mathrm{CD}$ & CLS \\
\hline CD or CLS only & 4.8 & 16.6 & 9.6 & No data & 9.5 & No data \\
\hline CD/CLS Mixture & \multicolumn{2}{|c|}{7.2} & \multicolumn{2}{|c|}{18.2} & \multicolumn{2}{|c|}{15.7} \\
\hline Preflush CLS, then CD & 4.8 & 16.6 & & .3 & & 9.9 \\
\hline
\end{tabular}

Table 4-6. Stability before and after Adsorption for Sandstone and Limestone with Starting Concentration of $500 \mathrm{mg} / \mathrm{l}$ of CD and $5000 \mathrm{mg} / \mathrm{l}$ of CLS

\begin{tabular}{|c|c|c|c|c|c|c|}
\hline & \multirow{2}{*}{\multicolumn{2}{|c|}{$\begin{array}{l}\text { Before } \\
\text { Injection }\end{array}$}} & \multicolumn{4}{|c|}{ Effluent Stream } \\
\hline \multirow{2}{*}{ Scheme } & & & \multicolumn{2}{|c|}{ Sandstone } & \multicolumn{2}{|c|}{ Limestone } \\
\hline & $\mathrm{CD}$ & CLS & $\mathrm{CD}$ & CLS & $\mathrm{CD}$ & CLS \\
\hline CD or CLS only & $\begin{array}{l}\text { Stable } \\
\text { foam }\end{array}$ & $\begin{array}{l}\text { No } \\
\text { foam }\end{array}$ & No foam & $\begin{array}{l}\text { No } \\
\text { data }\end{array}$ & No foam & \begin{tabular}{|l|} 
No \\
data
\end{tabular} \\
\hline CD/CLS Mixture & \multicolumn{2}{|c|}{ Stable foam } & \multicolumn{2}{|l|}{ No foam } & \multicolumn{2}{|l|}{ No foam } \\
\hline $\begin{array}{l}\text { Preadsorb (preflush) CLS, } \\
\text { then CD }\end{array}$ & $\begin{array}{l}\text { Stable } \\
\text { foam }\end{array}$ & \begin{tabular}{|l|} 
No \\
Foam
\end{tabular} & \multicolumn{2}{|c|}{$\begin{array}{l}50 \% \text { foam loss in } \\
90 \mathrm{~min}\end{array}$} & \multicolumn{2}{|c|}{$\begin{array}{l}90 \% \text { foam loss in } \\
90 \text { min }\end{array}$} \\
\hline
\end{tabular}

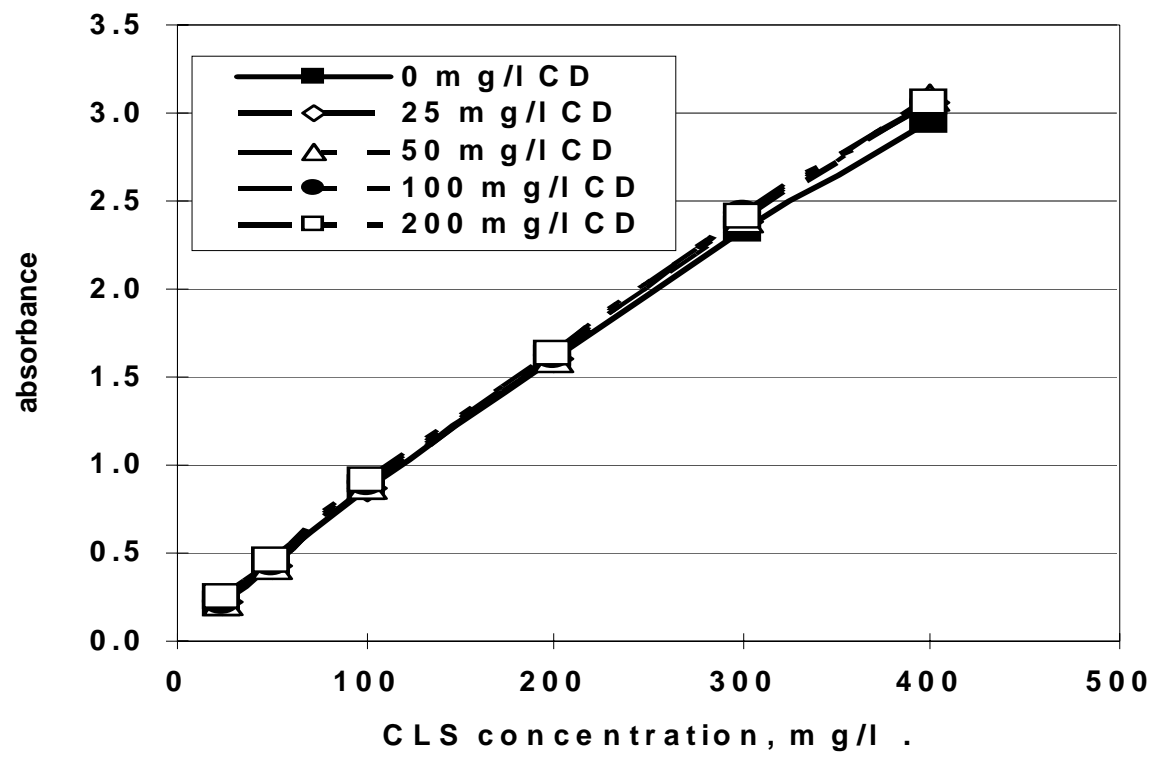

Fig. 4-1. Effect of CD on CLS absorbance at $283 \mathrm{~nm}$. 


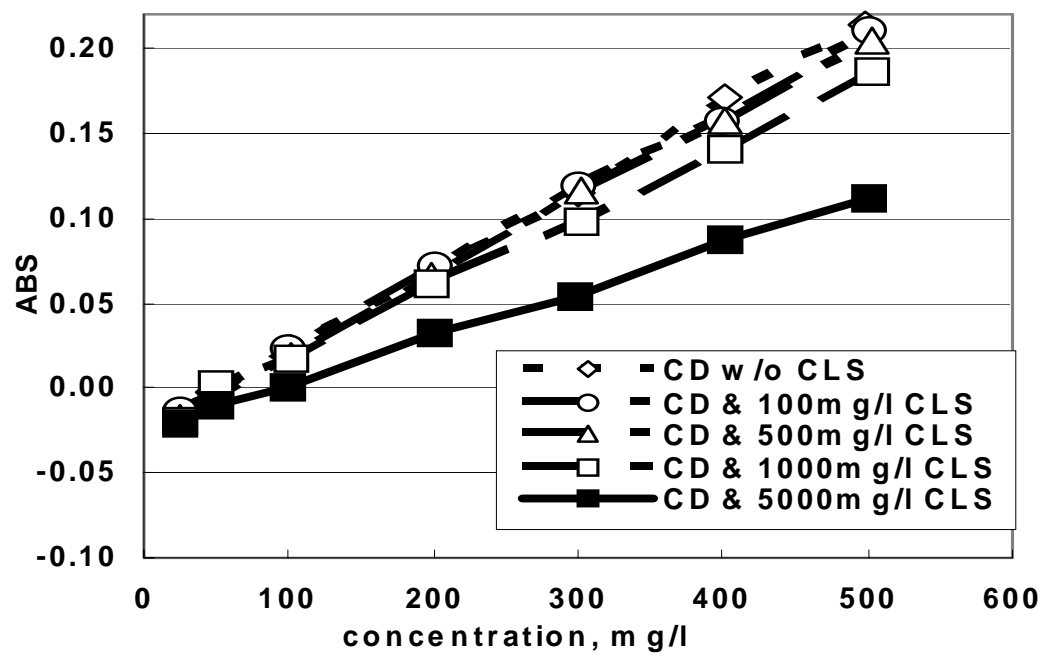

Fig. 4-2. CD standard curve at different concentration of CLS.

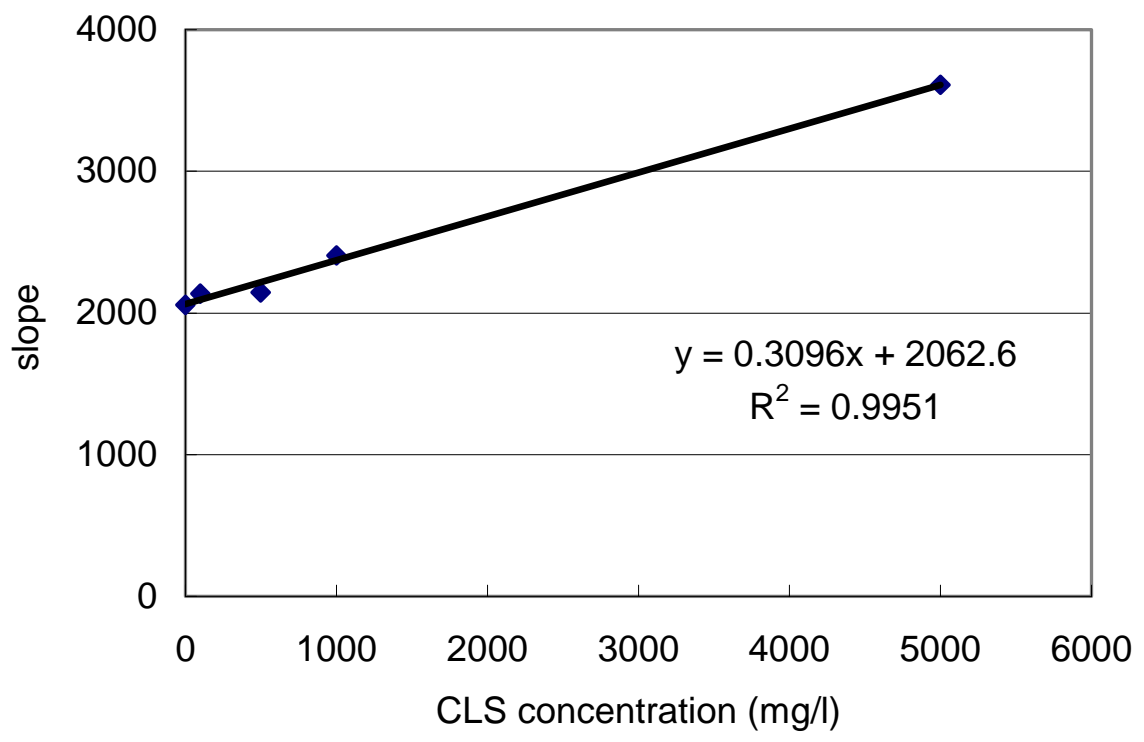

Fig.4-3. The relationship of CLS concentration and slope of fit equations. 


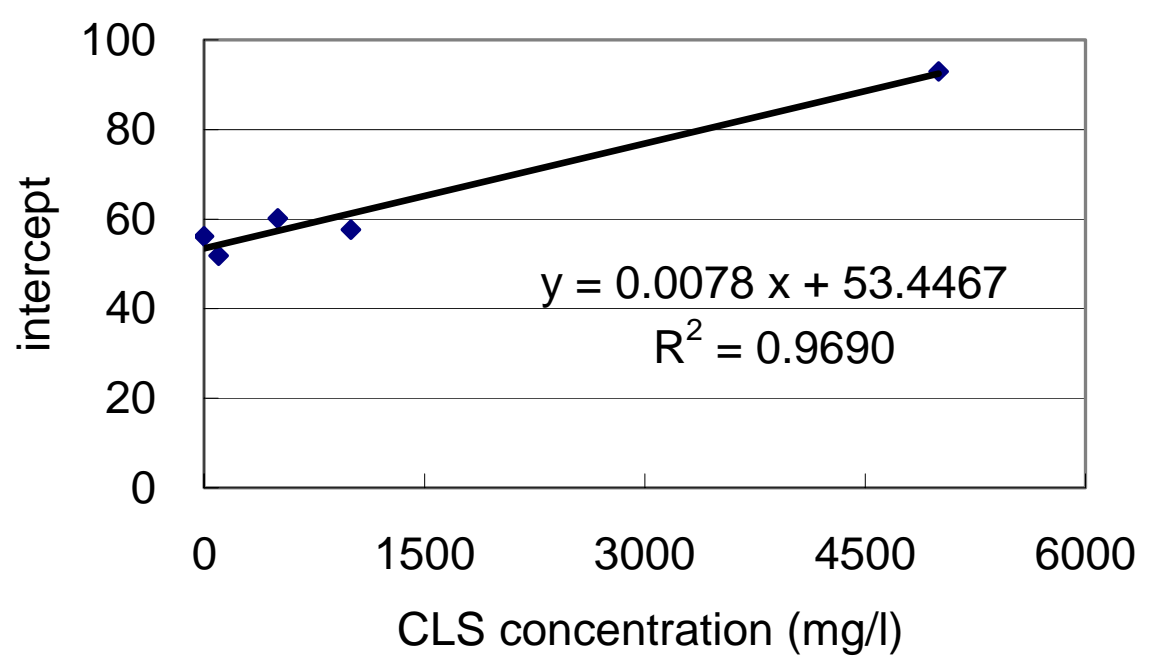

Fig.4-4. The relationship of CLS concentration and intercept of fit equations. 

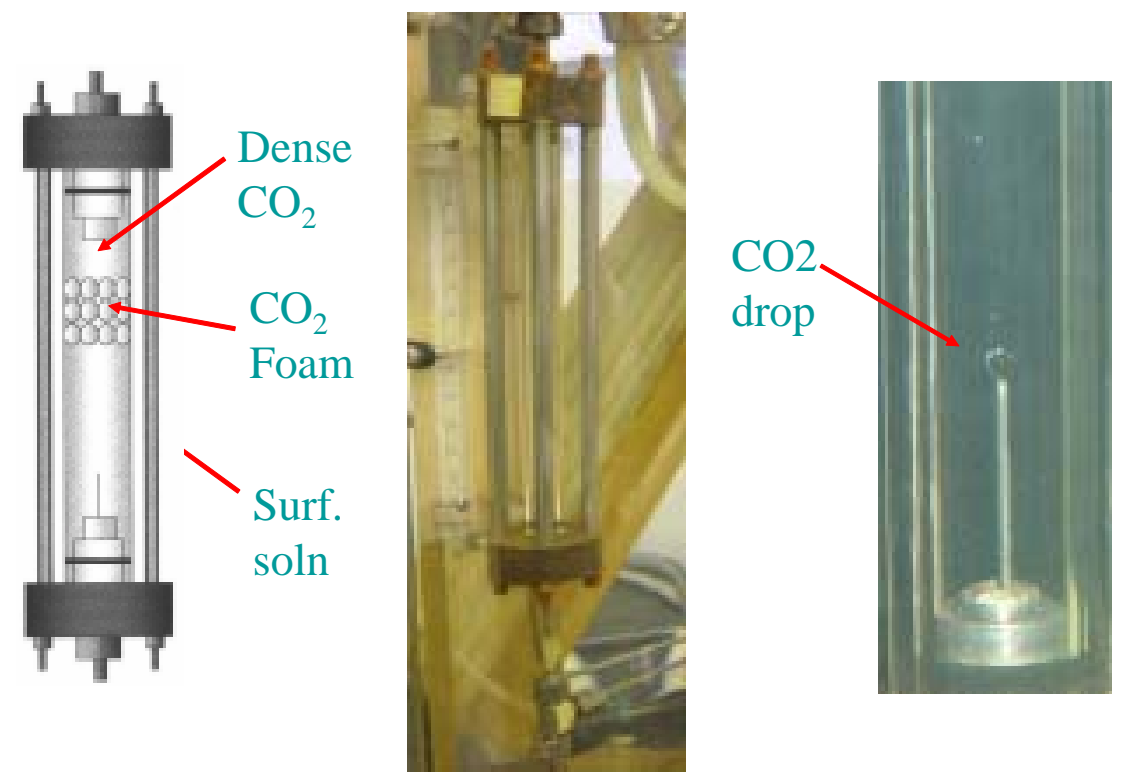

Fig. 4-5. The sapphire tube cell with capillary inlet.

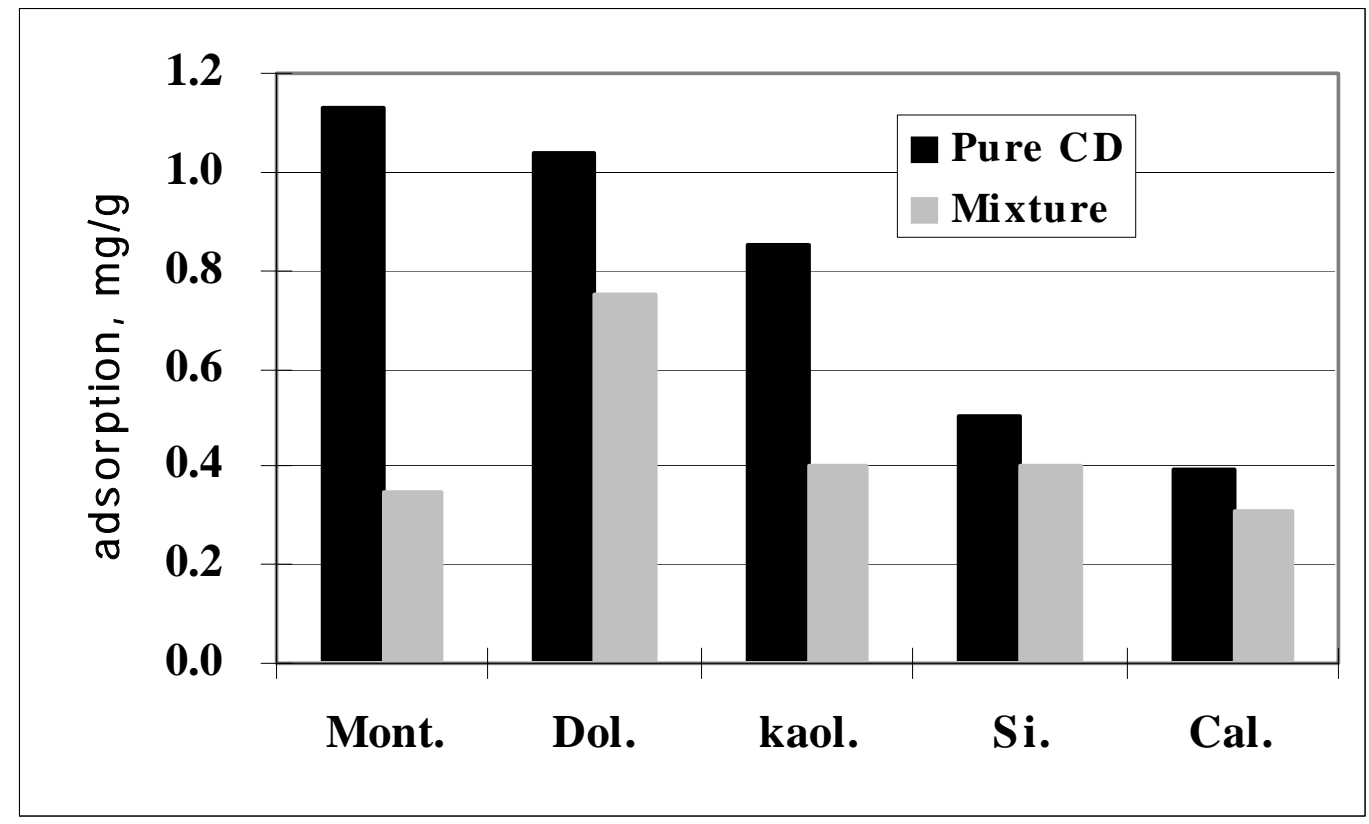

Fig. 4-6. CD adsorption density comparison for only $\mathrm{CD}$ and mixture (500 mg/l CD, 5,000 mg/l CLS, @ $40^{\circ} \mathrm{C}, 2 \%$ brine). 


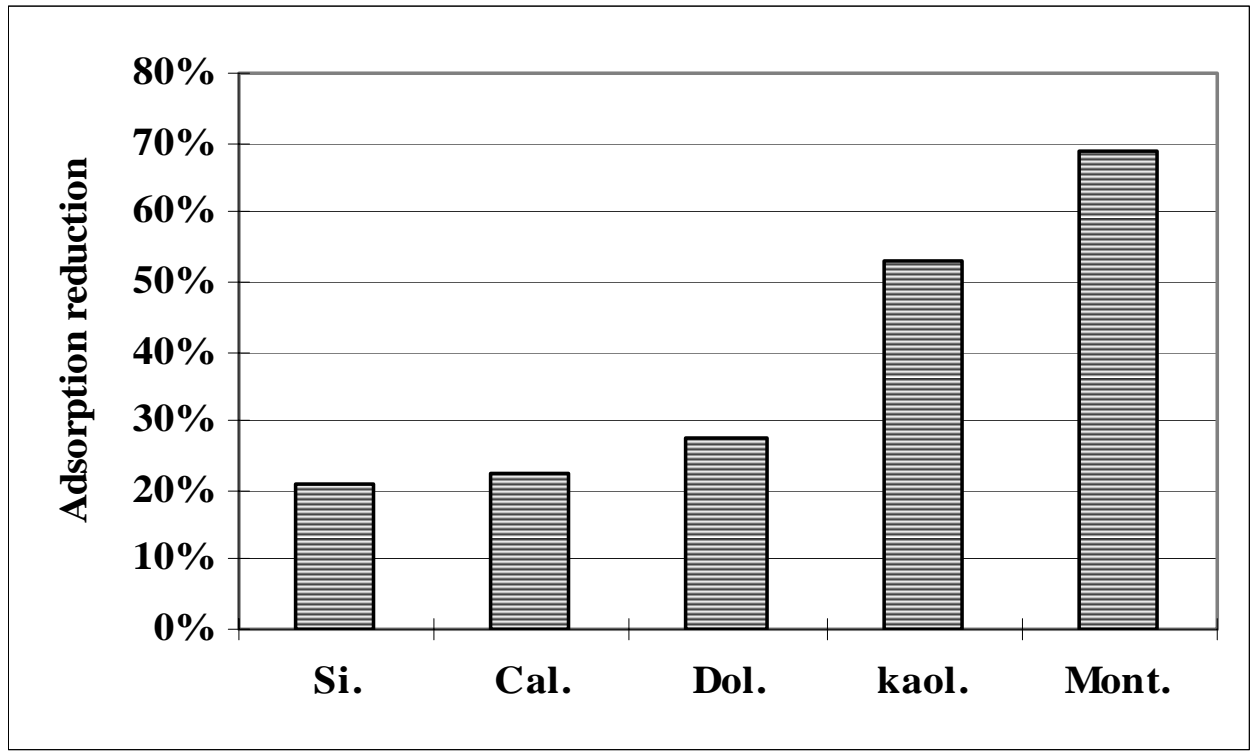

Fig. 4-7. CD adsorption reduction when mixing with CLS.

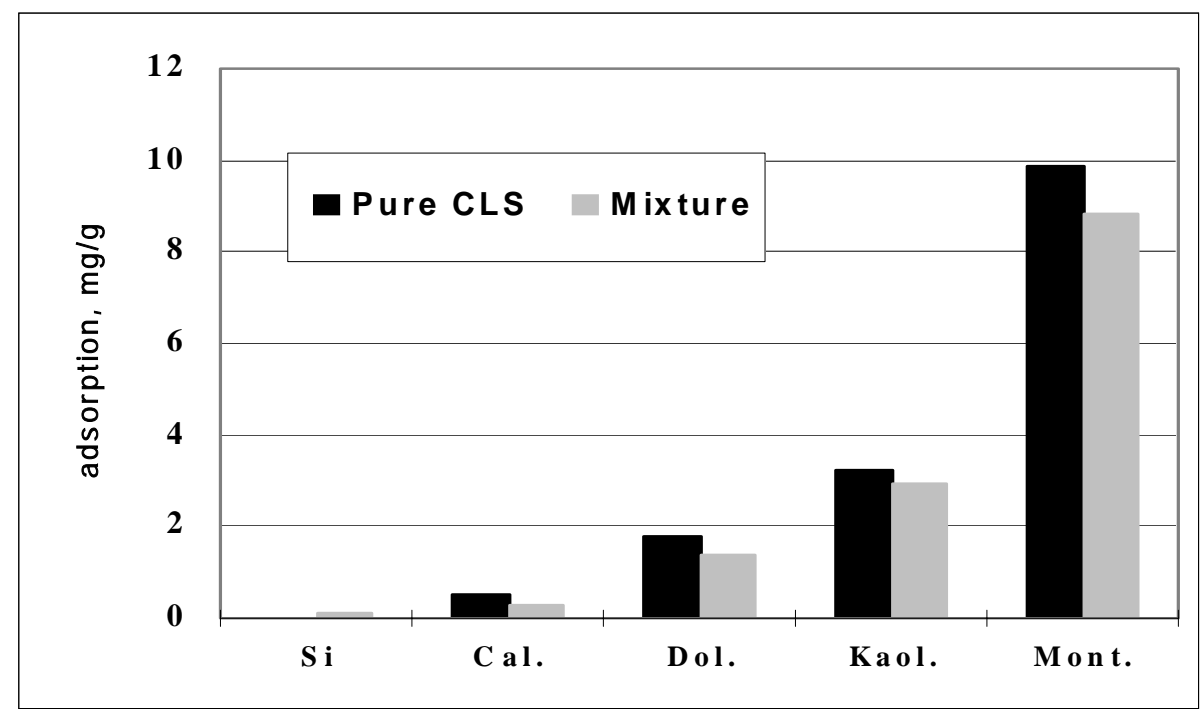

Fig. 4-8. CLS adsorption density comparison for CLS and mixture solution. 


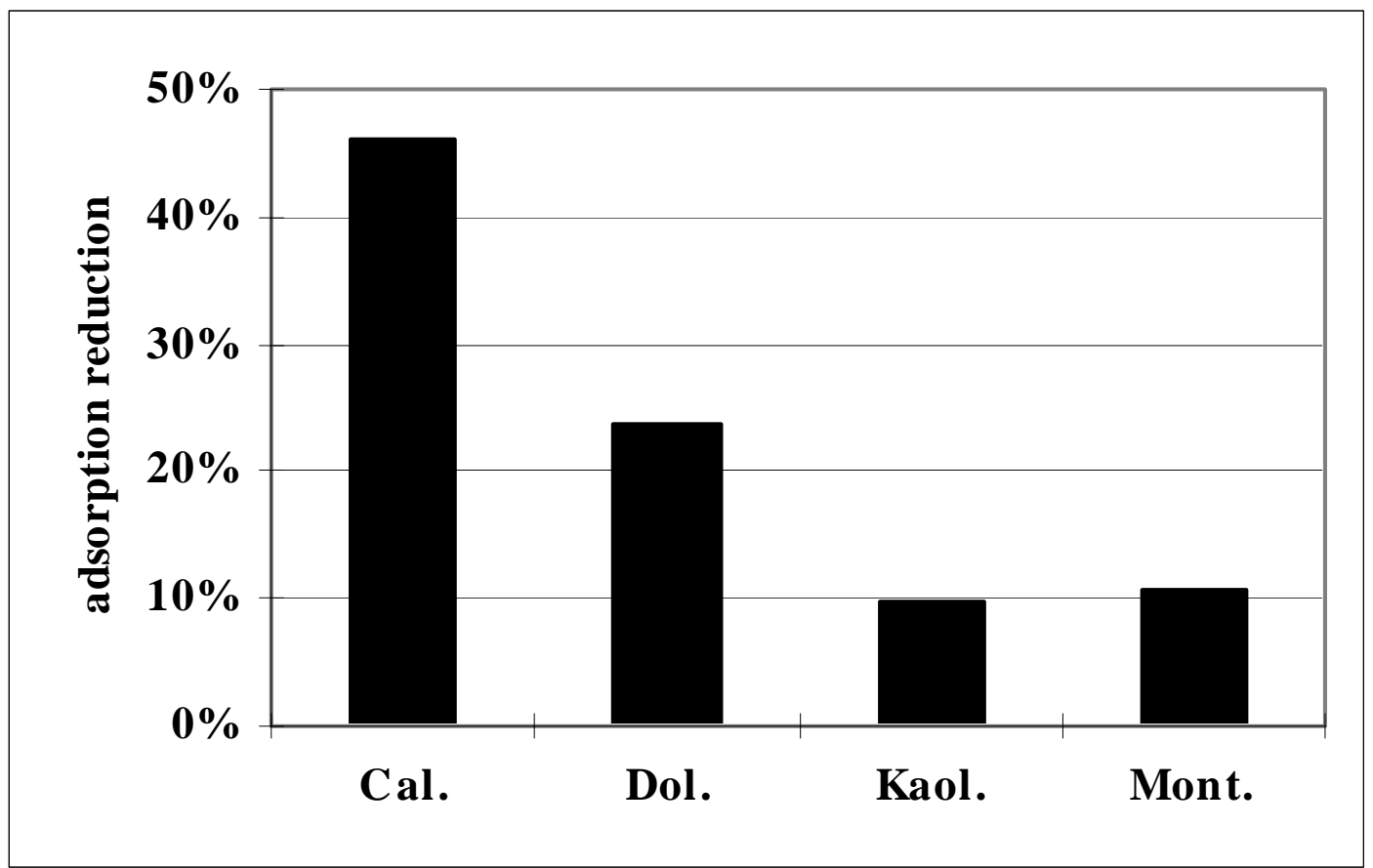

Fig. 4-9. CLS adsorption reduction when mixing with CD.

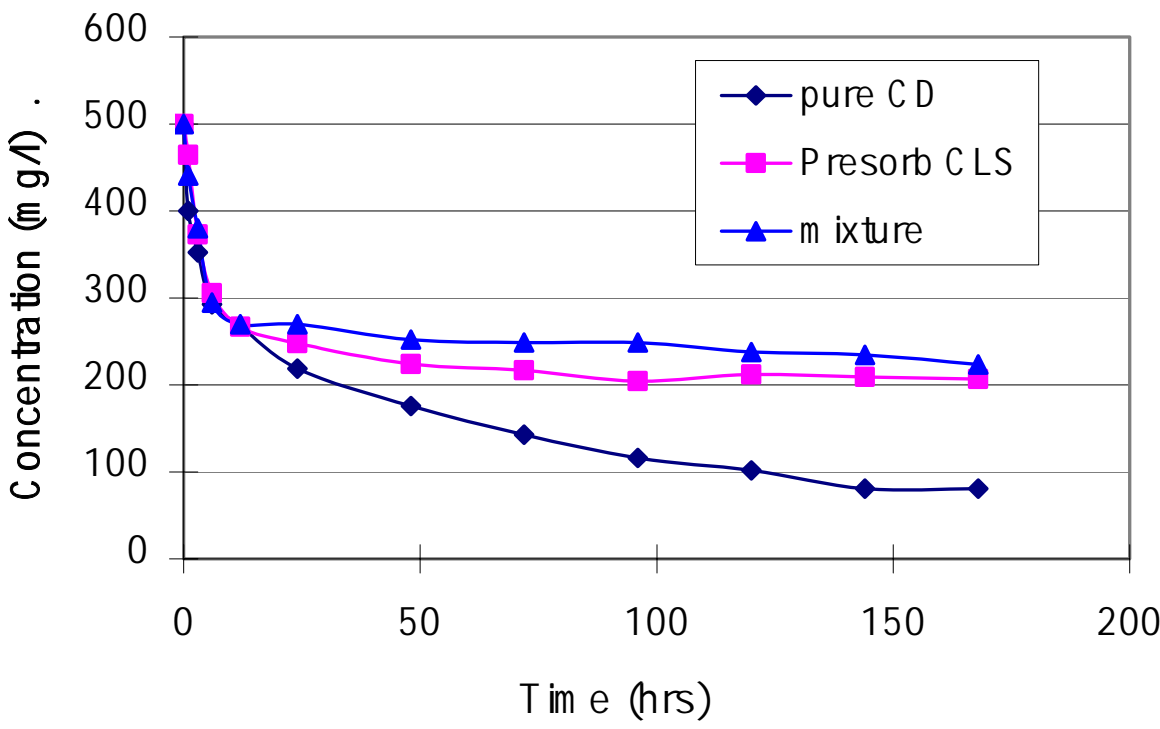

Fig. 4-10. CD concentration change versus time (sandstone). 


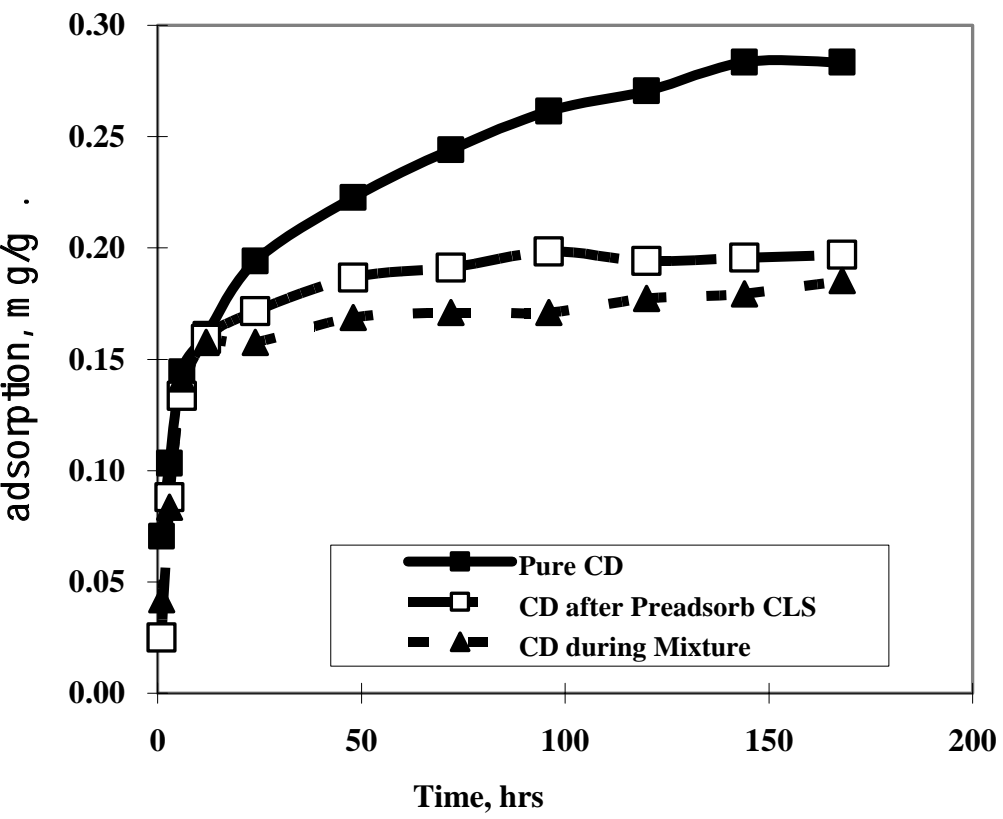

Fig. 4-11. CD adsorption onto sandstone versus time.

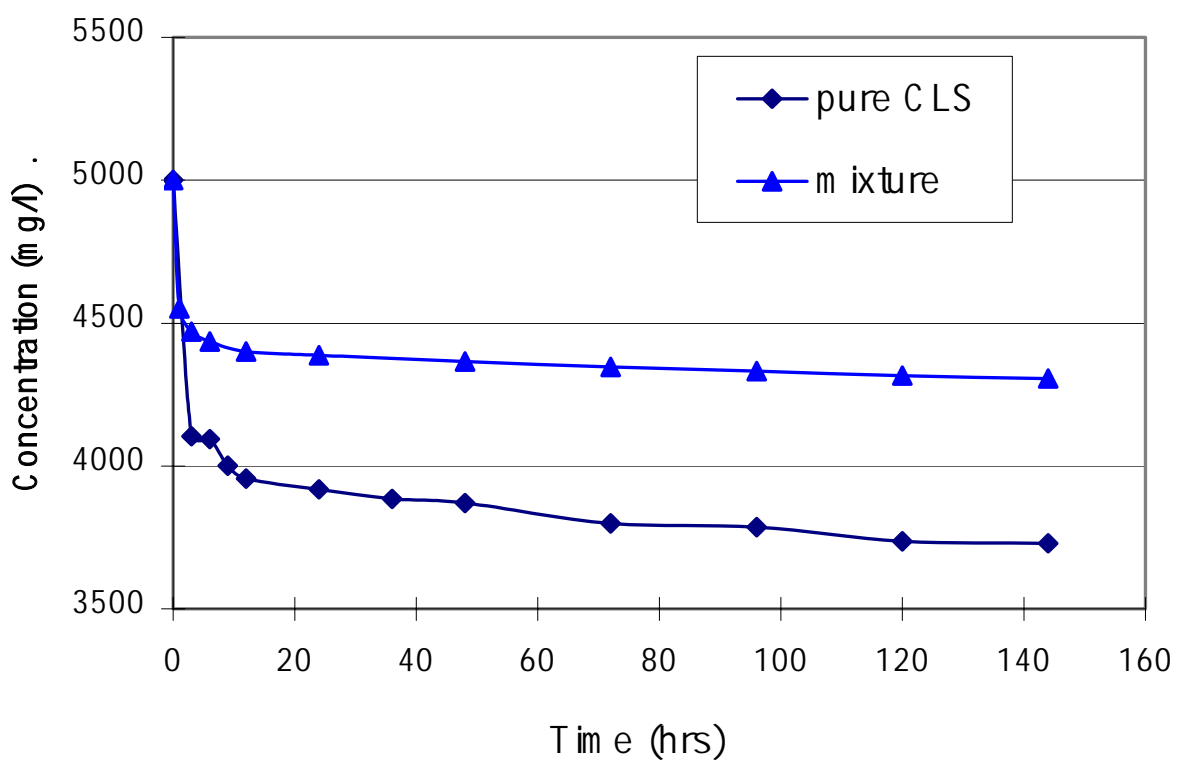

Fig. 4-12. CLS concentration change with time (Berea sandstone). 


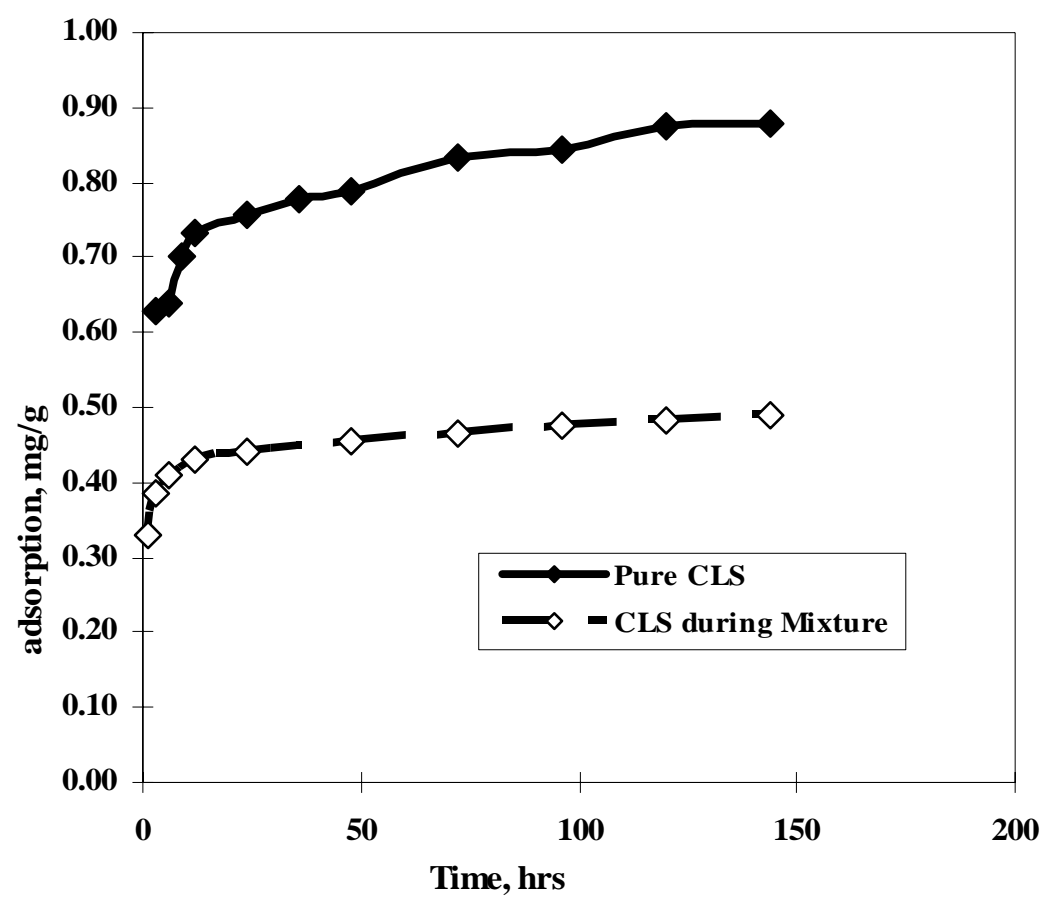

Fig. 4-13. CLS adsorption change with time onto sandstone.

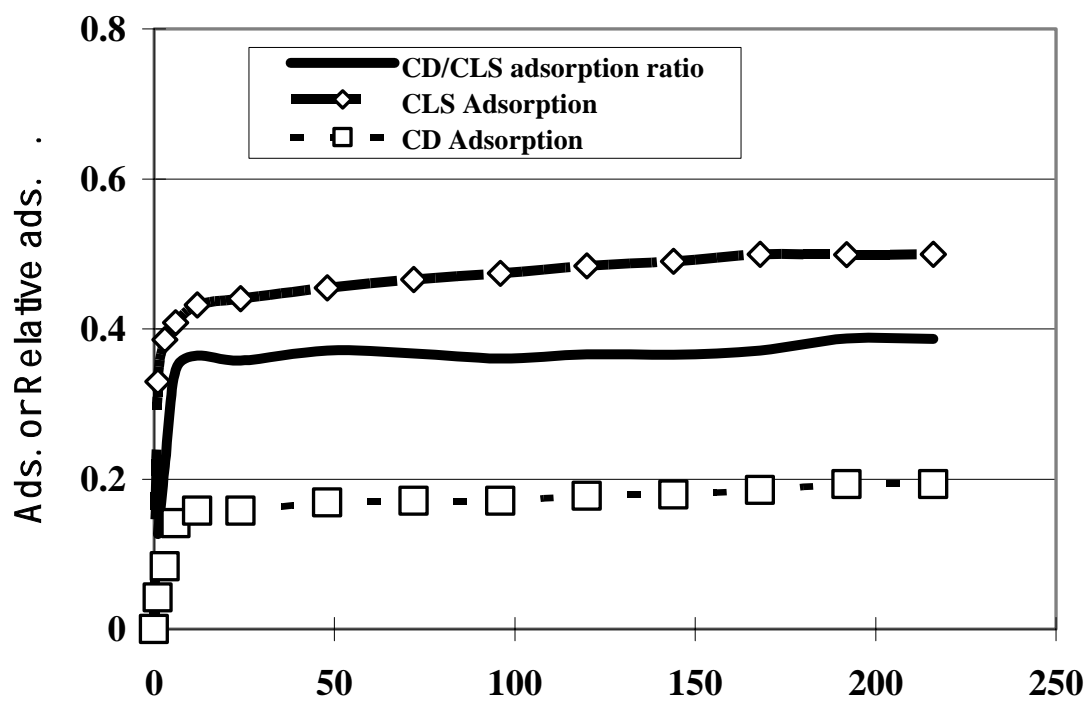

Time, hrs

Fig. 4-14. Adsorption profile comparison of CD and CLS during mixture injection. 


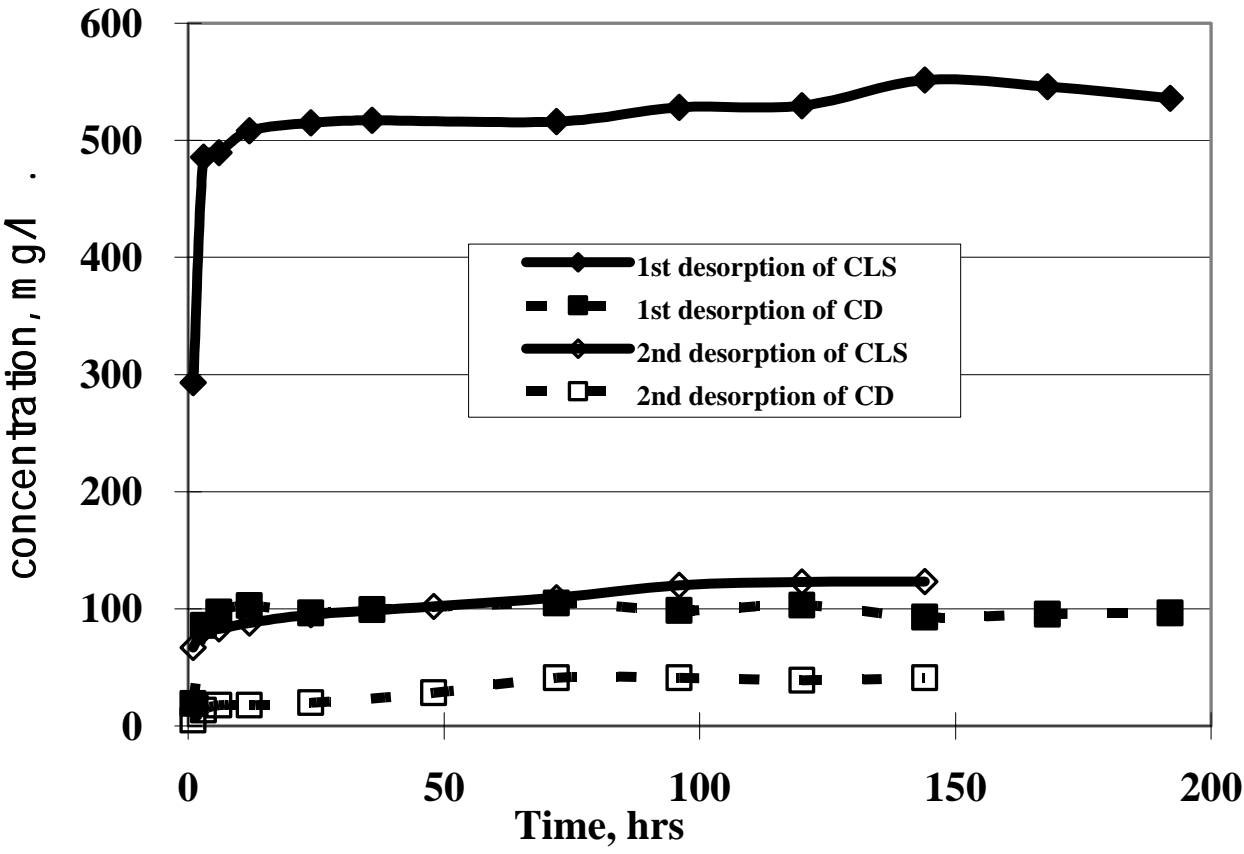

Fig. 4-15. Desorption profile comparison of CD and CLS after mixed injection.

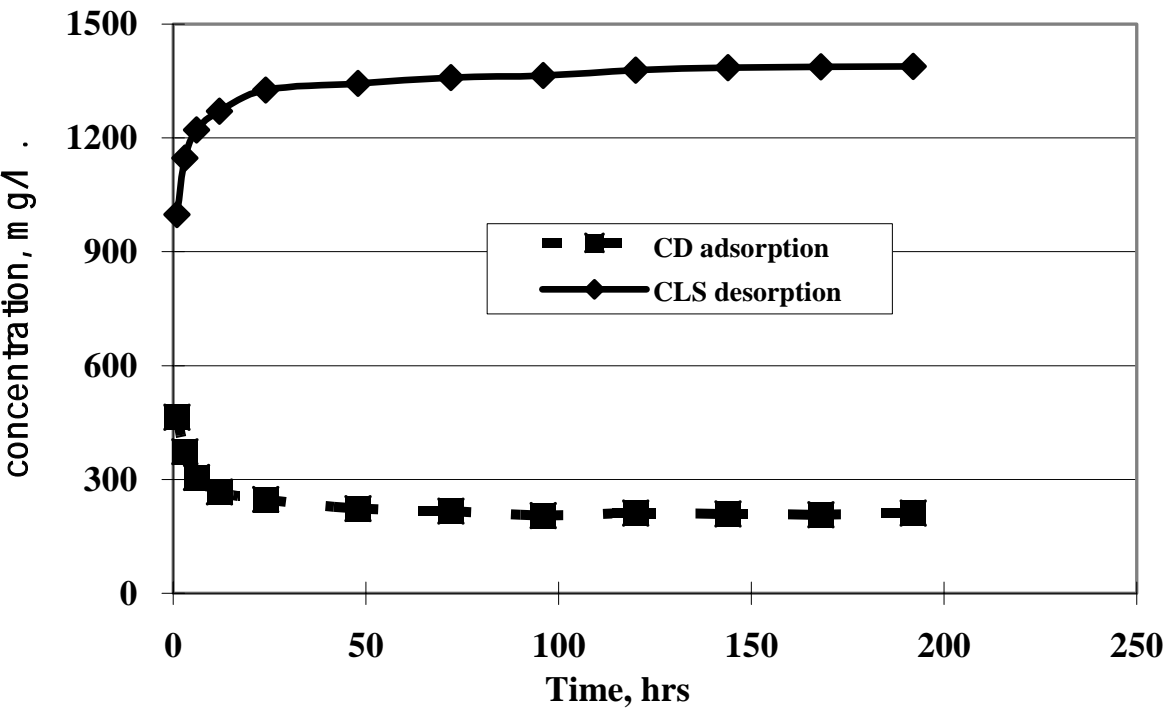

Fig. 4-16. CD adsorption and CLS desorption from Berea sandstone. 


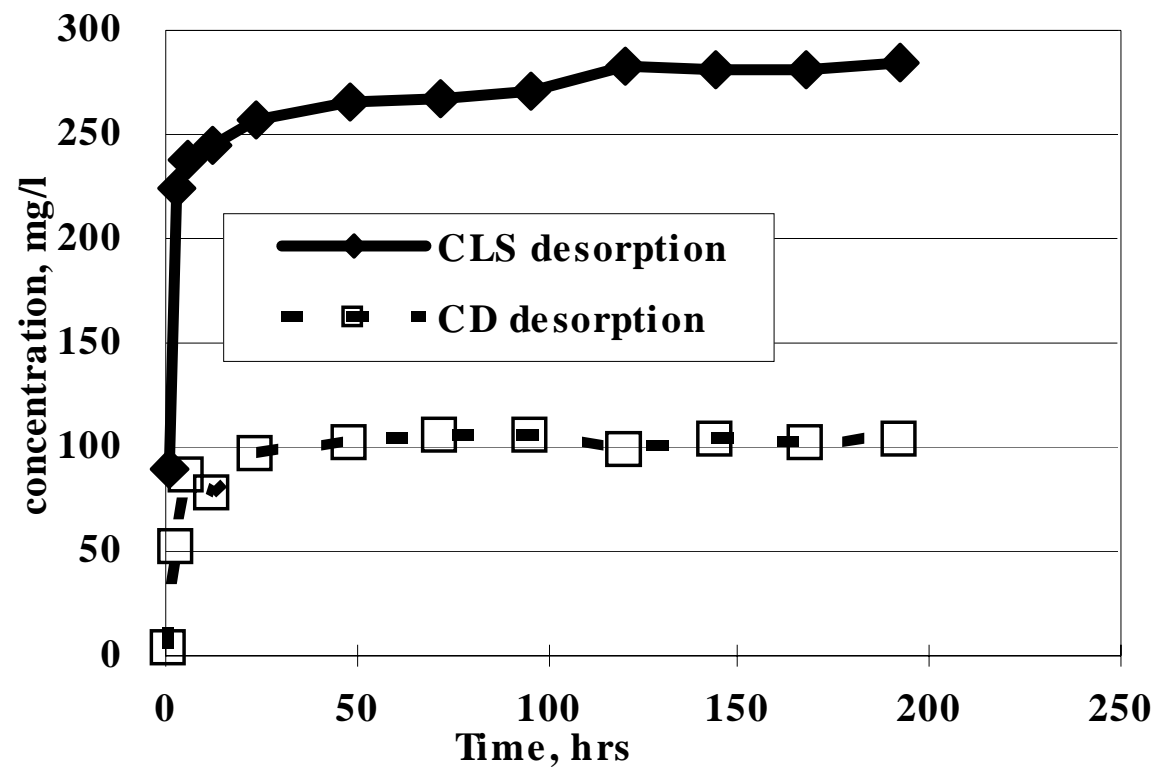

Fig. 4-17. CD and CLS desorption from Berea sandstone.

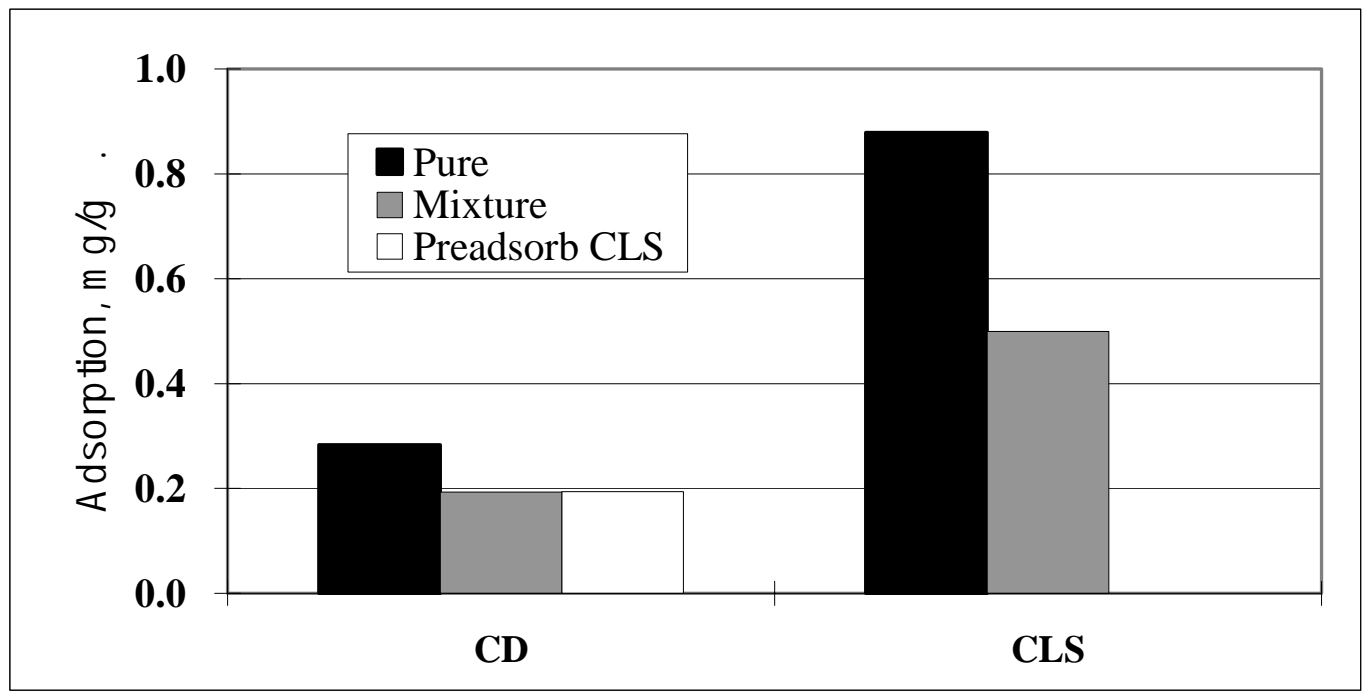

Fig. 4-18. Comparison of CD and CLS adsorption onto Berea sandstone for different schemes. 


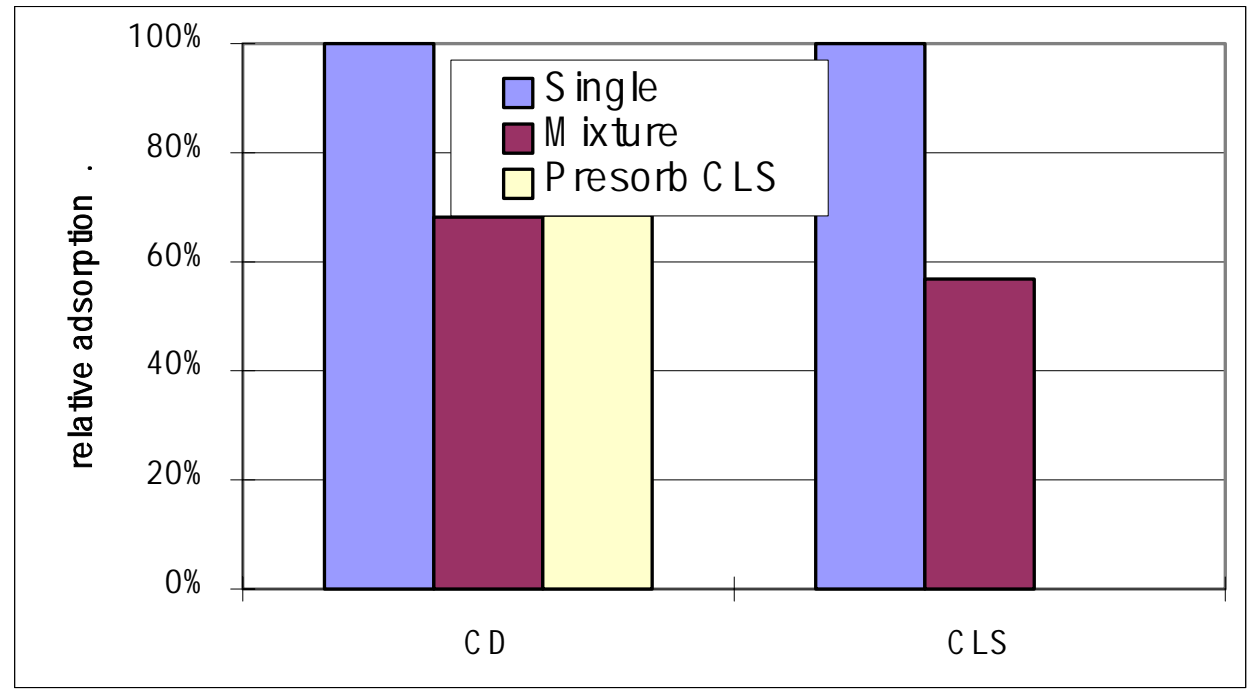

Fig. 4-19. Relative adsorption of CD and CLS onto Berea sandstone for different schemes.

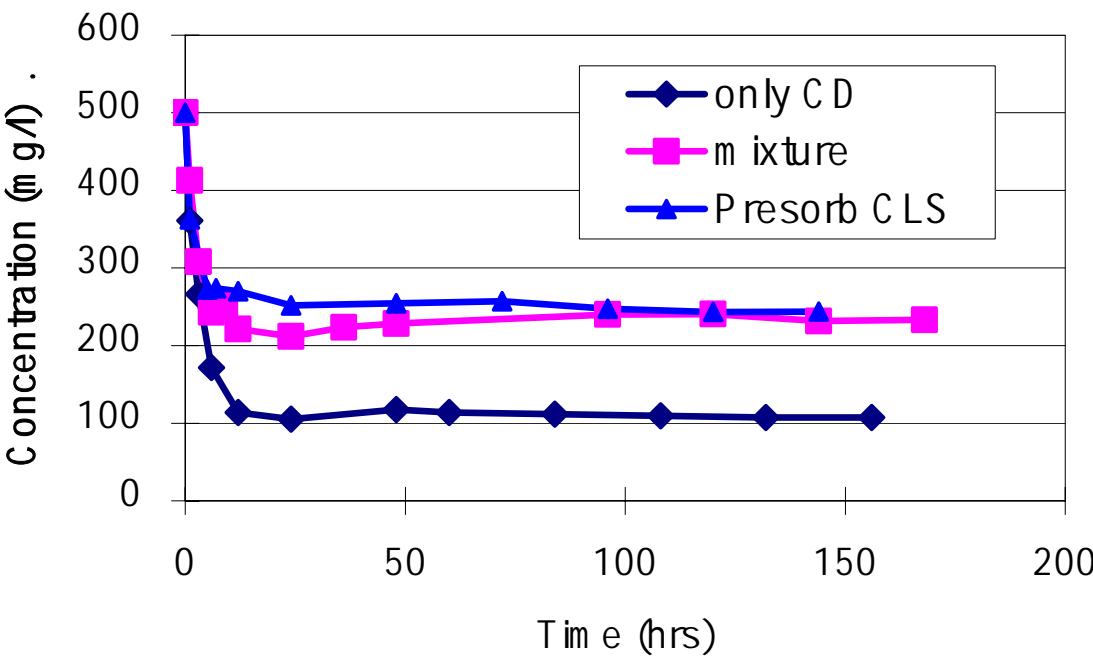

Fig. 4-20. CD concentration vs. time (limestone). 


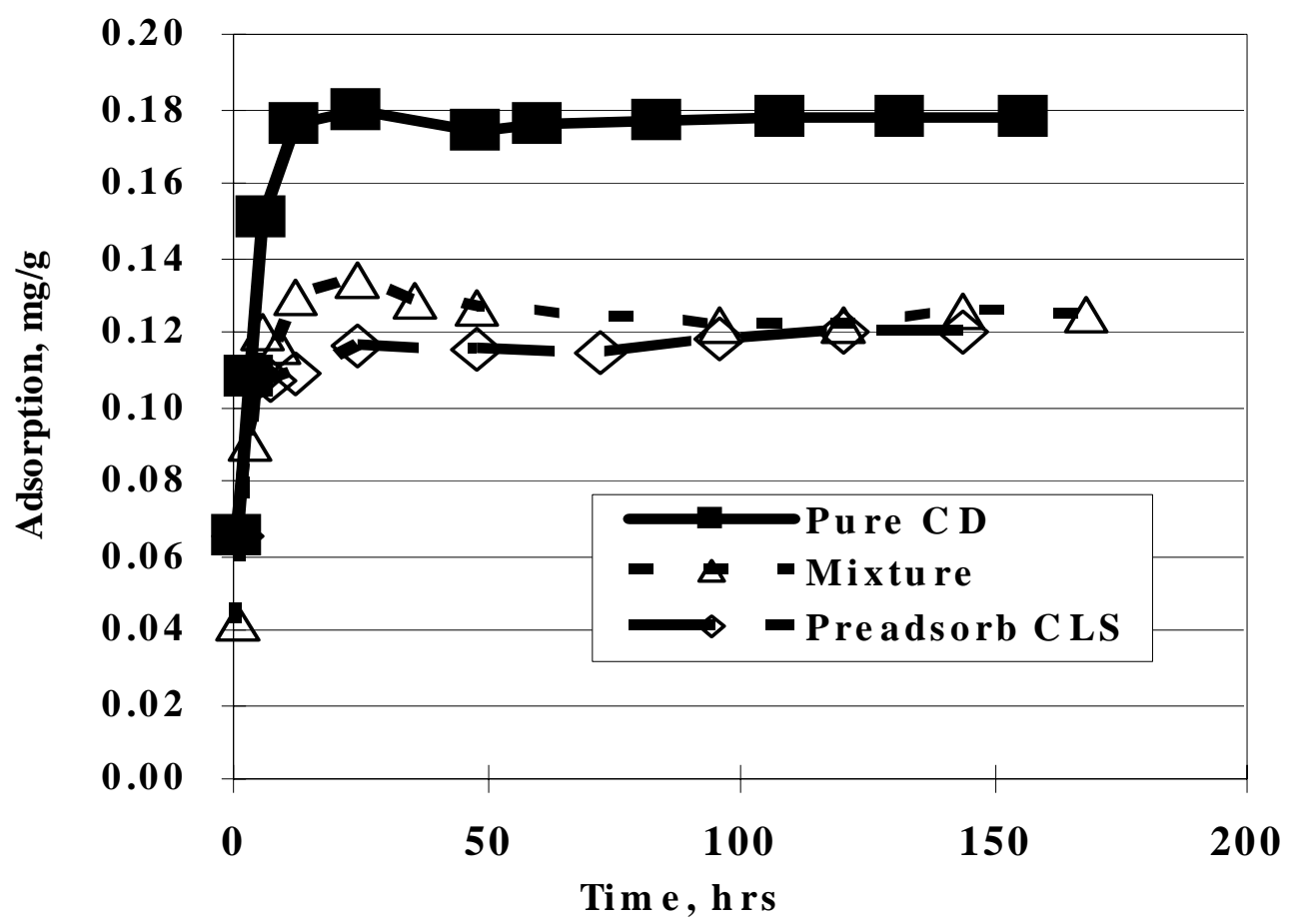

Fig. 4-21. CD adsorption vs. time onto limestone.

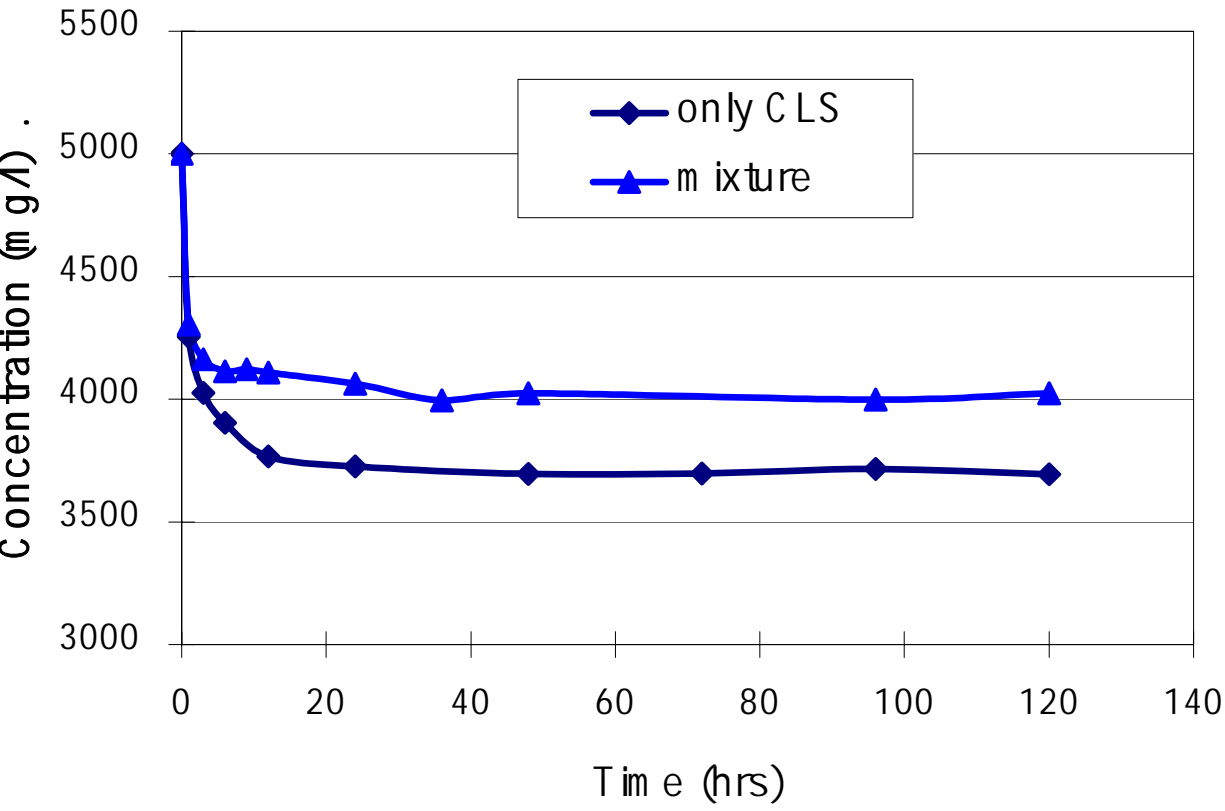

Fig. 4-22. CLS concentration vs. time (limestone). 


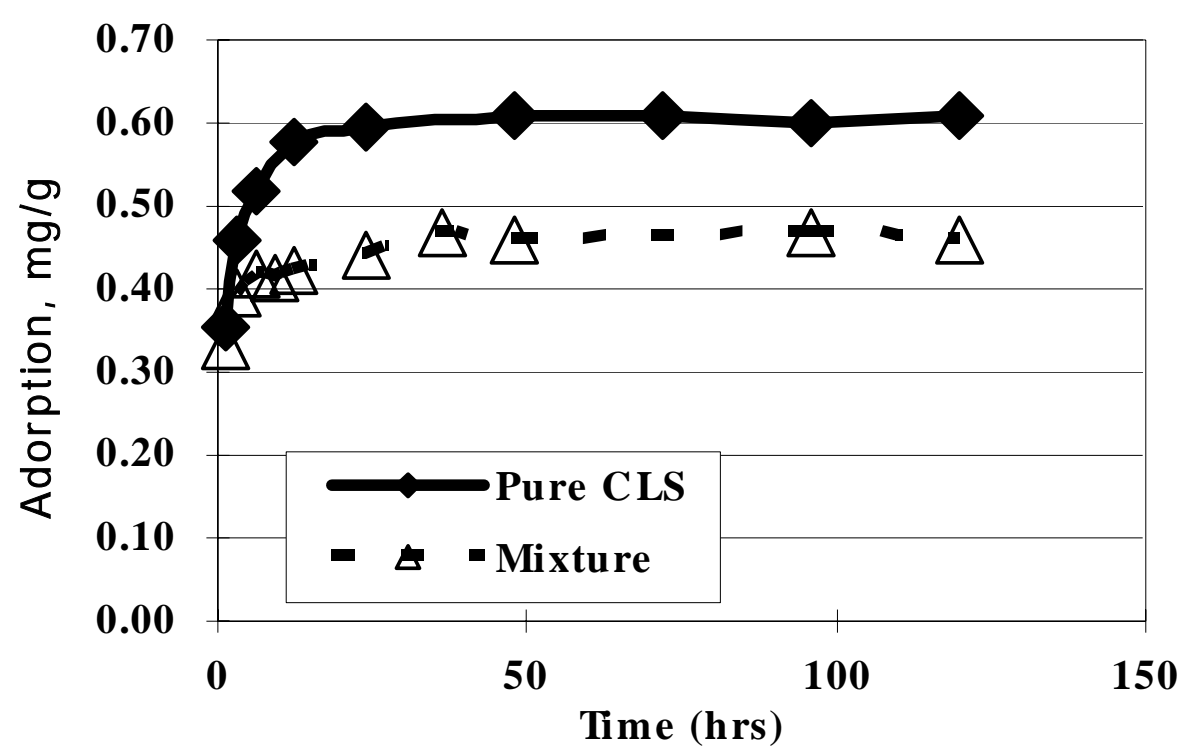

Fig. 4-23. CLS adsorption density vs. time onto limestone.

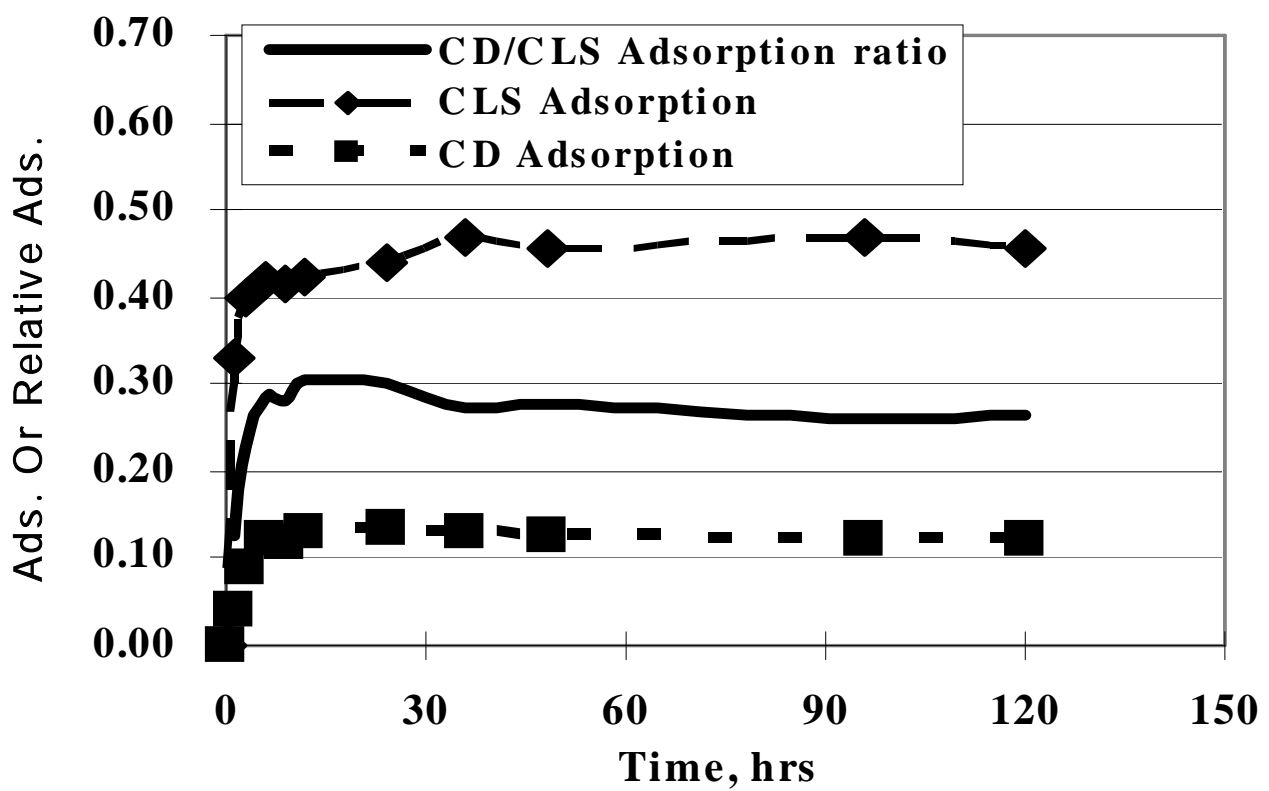

Fig. 4-24. Adsorption profile comparison of CD and CLS mixture on limestone. 


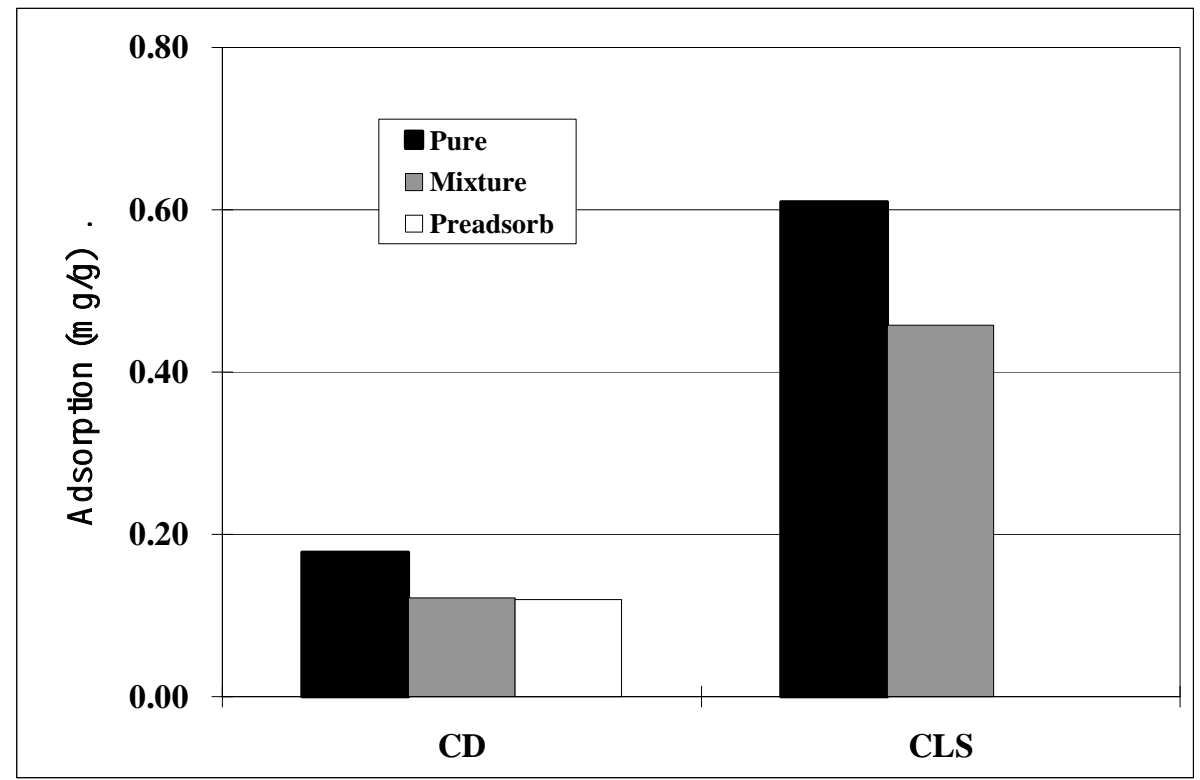

Fig. 4-25. Comparison of CD adsorption onto limestone using different schemes of injection.

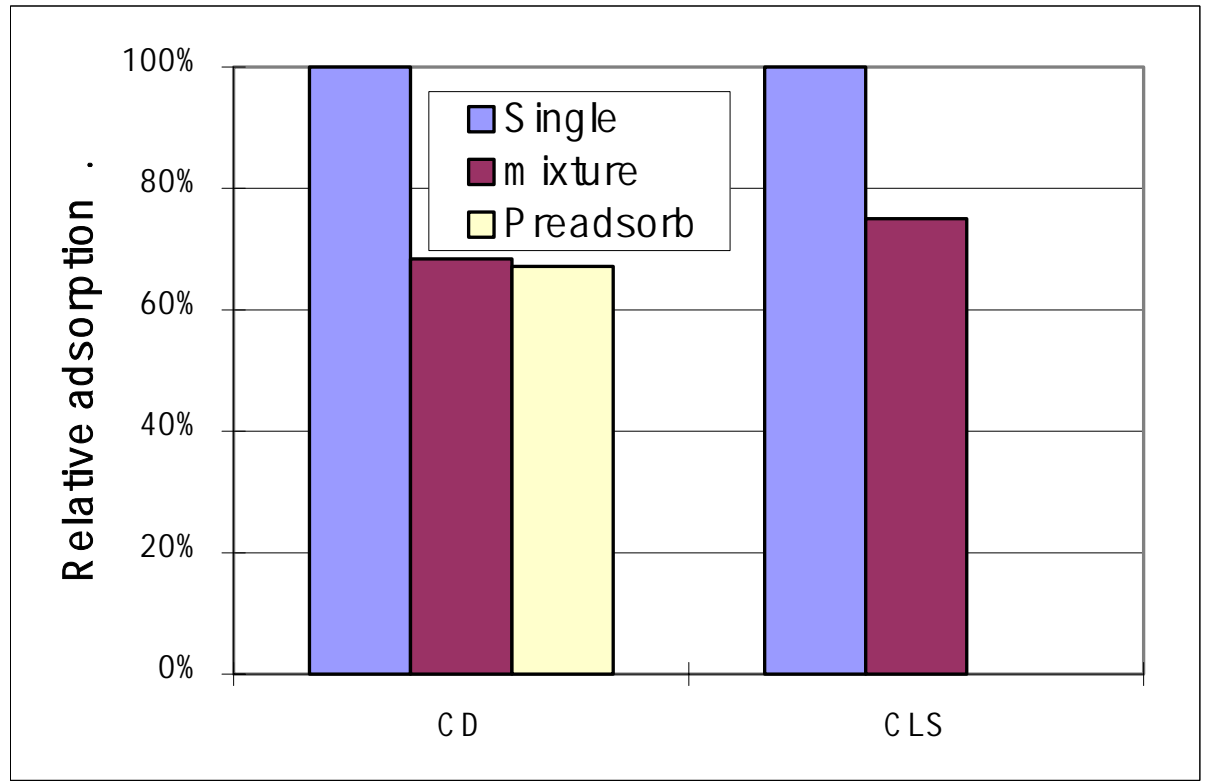

Fig.4-26. Relative adsorption of CD and CLS onto limestone. 

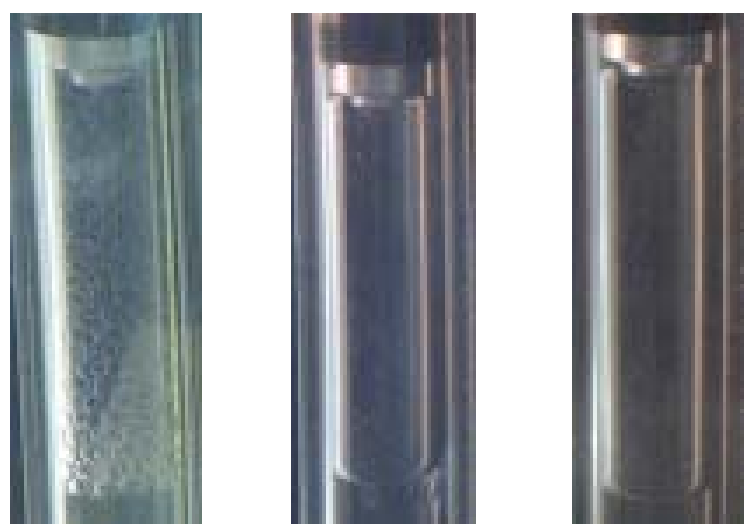

\section{$0.05 \%$ CD \\ Before \\ $0.05 \% \mathrm{CD}$ \\ 0.5\% CLS After 8 hour circulation Before}

Fig.4-27. Stability before and after adsorption in the limestone.
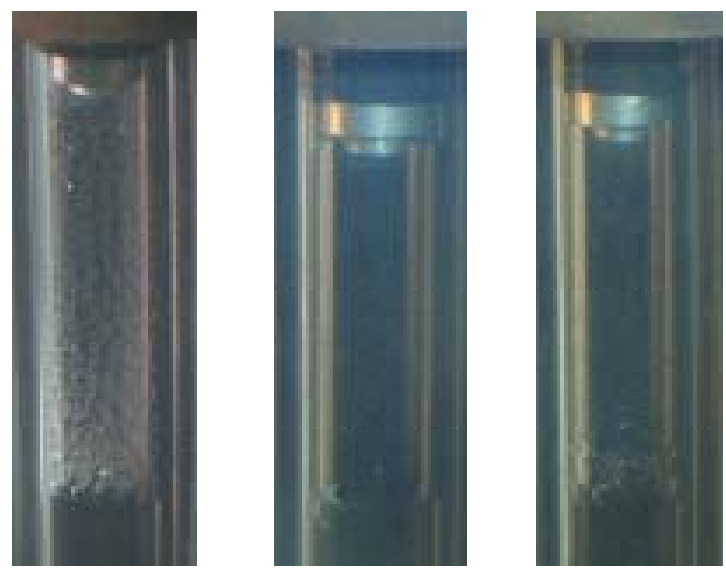
$0.5 \%$ CLS +
Preflush 0.5\% CLS
$0.5 \%$ CLS +
$0.05 \% \mathrm{CD}$
followed by $0.05 \% \mathrm{CD}$
$0.05 \% \mathrm{CD}$
After adsorption
After adsorption

Before adsorption

Fig.4-28. Stability before and after adsorption in the limestone for hybrid surfactant system. 

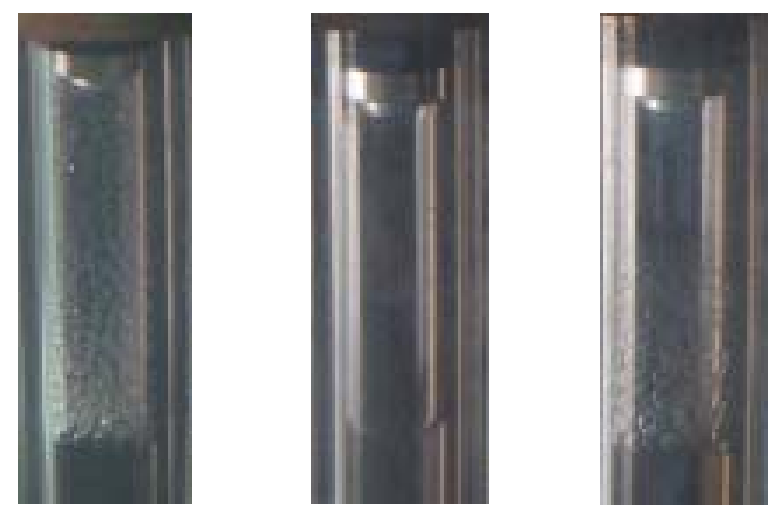

\section{5\% CLS + Preflush 0.5\% CLS $\quad 0.5 \%$ CLS + $0.05 \%$ CD followed by $0.05 \%$ CD $0.05 \%$ CD \\ Before adsorption After adsorption After adsorption}

Fig. 4-29. Stability before and after adsorption in the Berea sandstone for hybrid surfactant system. 


\title{
CHAPTER 5. INJECTIVITY LOSS: DEVELOPMENT OF GENERAL EQUATIONS TO QUANTIFY STRESS-SENSITIVITIES OF PERMEABILITY AND NON-DARCY COEFFICIENT
}

\begin{abstract}
Non-Darcy flow parameters, i.e., permeability, $\mathrm{k}$, and the non-Darcy coefficient, $\beta$, were investigated in 159 series of high pressure/high temperature/high velocity gas flooding experiments on five different rock samples under field reservoir conditions. The results of these experiments reconfirm and extend to new conditions that permeability decreases, while the nonDarcy flow coefficient increases with effective stress, $\sigma_{\text {eff; }}$ further, both are independent of shear stresses. Linear correlations bewteen non-Darcy flow parameters and effective stress have been developed in terms of nominal non-Darcy parameters, which are the non-Darcy parameters at zero effective stress. Stress-sensitivities, $s_{k}$ and $s_{\beta}$, each defined as the coefficient in the $k-\sigma_{\text {eff }}$ and $\beta-\sigma_{\text {eff }}$ linear correlation, are further found to be proportional to the nominal non-Darcy parameters regardless of rock property and reservoir conditions. General equations to quantify stress-sensitivities of permeability and non-Darcy coefficient have been developed in terms of nominal non-Darcy flow parameters. Using these general equations and correlations, the change of permeability and non-Darcy coefficient can be calculated under given reservoir conditions, which provides a way to estimate injectivity and productivity losses at the near-wellbore region.
\end{abstract}

\section{Introduction}

Reservoir performance changes when the reservoir pressure increases or decreases. This performance change is more severe in stress-sensitive formations such as the Mesa Verde formation in northwestern Colorado, ${ }^{1}$ the Spraberry formation of west Texas, ${ }^{2}$ and the Ekofisk reservoirs of North Sea. ${ }^{3}$ Lorenz $^{4}$ has performed a comprehensive review on stress-sensitive reservoirs.

There are a number of publications on the investigation of stress effects on permeability under Darcy flow conditions. ${ }^{5,6}$ However, it is well known that non-Darcy behavior also has significant influences on well performance, especially at the near-wellbore region in gas wells, both producers and injectors. ${ }^{7}$ Many papers have been published on the determination of non-Darcy 
flow parameters, i.e., permeability and the non-Darcy flow coefficient, the physical meaning of the non-Darcy flow coefficient, and the correlations between permeability and the non-Darcy flow coefficient. ${ }^{8,9}$ Some numerical simulations have included the non-Darcy effect. ${ }^{10}$ However, changes in the non-Darcy flow parameters with pressures - thus in the effective in-situ stresseshas heretofore not been considered. A better characterization and prediction of reservoir dynamics requires the correlation between non-Darcy flow parameters and effective stresses, and furthermore, the stress sensitivities of these parameters. ${ }^{11}$

Several studies have addressed the influence of overburden pressure on the non-Darcy flow coefficient. ${ }^{12,13}$ In one recent study, ${ }^{14}$ several correlations between non-Darcy flow parameters and effective stress were noted, indicating that permeability decreases while the non-Darcy flow coefficient increases linearly with effective stress. On the other hand, shear stress was shown to have negligible correlations with non-Darcy flow parameters. These conclusions were drawn from experiments on a specific rock, Dakota sandstone, under specific experimental conditions $\left(100^{\circ} \mathrm{F}, 500\right.$ psi outlet pressure $)$.

The applicability of these conclusions to different rocks under different formation temperatures and pore pressures needs further investigation. In addition, general formulas are needed to judge the stress-sensitivity of the non-Darcy flow parameters of any given rock, and further to predict the change of these parameters under given in-situ stress conditions. Based on the results of 159 series of high pressure/high temperature/high velocity (HP/HT/HV) gas flow experiments, this chapter presents the validation and extension of the previous conclusions; and the development of the general equations for calculating stress-sensitivity of any given rocks, and for predicting the non-Darcy flow parameters under given in-situ stress fields.

\section{Experimental}

\section{High Pressure / High Temperature / High Velocity Gas Flooding Experiments}

Field Conditions. In order to reflect the representative conditions of real-world fields in the experiments, a survey was conducted concerning the conditions of active $\mathrm{CO}_{2}$ flooding projects. From these surveys it was found that: ${ }^{15,16}$

1. Among the $75 \mathrm{US}$ active $\mathrm{CO}_{2}$ flooding projects surveyed, $61 \%$ of the $\mathrm{CO}_{2}$ floods are in formations at depths between 4000 and $6000 \mathrm{ft}$, with 5\% in formations deeper than $10000 \mathrm{ft}$. 
2. The majority $(71 \%)$ of the $\mathrm{CO}_{2}$ flooded formations have a temperature between $100^{\circ} \mathrm{F}$ and $150^{\circ} \mathrm{F}$, with $5 \%$ over $200^{\circ} \mathrm{F}$.

3. The majority (53\%) of the flooded formations have permeability less than $10 \mathrm{mD}$, while $33 \%$ are 10 to $100 \mathrm{mD}, 8 \%$ are 100 to $500 \mathrm{mD}$, and $5 \%$ are higher than $500 \mathrm{mD}$.

4. The majority $(60 \%)$ of the flooded formations have porosity fractions of 0.10 to $0.20,19 \%$ are 0.20 to $0.30,15 \%$ are 0.05 to 0.10 , and $5 \%$ are less than 0.05 .

5. Three representative $\mathrm{CO}_{2}$ flooding projects reviewed earlier ${ }^{15}$ have superficial velocity in the near-wellbore region from 21 to $1170 \mathrm{ft} / \mathrm{day}$.

Based on the above survey results and laboratory capability, five different rocks have been selected and tested under the experimental conditions shown in Table 5-1.

Development of Experimental Facilities, In order to fulfill the research objectives under the experimental conditions shown in Table 5-1, a previous HP/HT/HV gas flooding system was upgraded. ${ }^{15}$

The whole system was upgraded so that all manual operations were to be conducted in open space at room temperature. This included switches of all the valves, pressure gauges and meters, temperature meters, injection pump controller, displacement pump, and the gas source tank (Fig. 5-1). The injection pump was set in an outer (larger) air bath, in which the gas was to be heated to $80^{\circ} \mathrm{F}$ (the limit of the injection pump). The gas was designed to be pumped into an inner (smaller) air bath to be further heated to the desired higher temperature (with a limit of $200^{\circ} \mathrm{F}$ ), and then to flow through the inlet back pressure regulator (BPR), the core, the outlet BPR, and to the atmosphere. Thermal equilibrium of the whole system was assured by five thermocouples at different locations: (1) the cylinder of the injection pump, (2) the center of the inner air bath, (3) the inlet of the coreholder, (4) the middle of the coreholder, and (5) the outlet of the coreholder.

The test apparatus was designed, assembled, and calibrated for determining permeability and non-Darcy flow coefficient under varying conditions of overburden pressures $(0 \sim 10000 \mathrm{psi})$, pore pressure $(0 \sim 3000 \mathrm{psi})$, axial and radial stresses $(0 \sim 10000 \mathrm{psi})$, temperatures (room temperature $\left.\sim 200^{\circ} \mathrm{F}\right)$, and flow rates $\left(0 \sim 10000 \mathrm{cc} / \mathrm{hr}\right.$ at $80^{\circ} \mathrm{F}$ and $\left.2000 \mathrm{psi}\right)$. Digital devices 
were used to display the differential pressure and temperatures. Heating/cooling coils were installed to speed up thermal equilibrium. Venting/circulating fans were added for homogeneous distribution of temperature in the air baths. The equipment has been tested and calibrated to the rated pressures and temperatures to ensure safe operation. It has a more accurate data acquisition system and better thermal control than before.

\section{Core Sample Preparation}

Five core samples from five different rocks, i.e., tight Dakota sandstone (DSS201), low permeability Indiana limestone (IL301), high permeability Indiana limestone (IL302), low permeability Berea sandstone (BSSL301), and high permeability Berea sandstone (BSSH301), were prepared for the experiments. The samples were approximately 1-in. in diameter and 2-in. long. First these core samples were placed in a coreholder and cleaned using 5 10 pore volumes (PV) of tetrahydrofuran (THF) under a sufficient radial pressure to ensure that THF flowed through the core. Then these cores were dried in the oven at $150^{\circ} \mathrm{F}$ for 24 hours to remove any residual water and THF. Table 5-2 lists the specifications of the core samples used for the experimental work.

The core sample was then installed into a triaxial core holder capable of applying independent radial and axial pressures as well as pore pressures to the sample. The core sample was held within a nitrile rubber sleeve by confining or radial pressure. Independent axial pressure was applied with an axial loading valve. Inlet and outlet valves allowed fluids to be injected through the core sample. Details of the core assembly have been given elsewhere. ${ }^{15}$

\section{Pressurization}

First, the annulus around the rubber sleeve was filled with distilled water. Then, axial stress was applied up to about 500 psi. This eliminated any gap between the distribution plug and the core face, which could rupture the sleeve if axial pressure was applied first. Then radial stress was

applied to the pre-set value, and axial stress again was increased to the pre-set value. Finally pore pressure was applied to the core, and flooding process began.

Depressurization followed the opposite order. Incidentally, care must be taken in pressurization 
and depressurization so that radial stress is always several hundred psi higher than the maximum pore pressure in the core so as to prevent sleeve damage.

\section{Porosity Measurement}

Core porosity is an important parameter required for the proper analysis of the experimental work. A special attachment was fabricated and used to measure the porosity of the core samples. The test apparatus was initially flushed with THF and then flushed with nitrogen. Pressures in the inlet and outlet BPR's were set to 2000 psi and 500 psi, respectively. A calibration core was prepared from brass stock, and placed carefully in the coreholder. The assembly was then connected to the test apparatus. The calibration core was subjected to an initial pressure using nitrogen gas. All volumes were determined before the test. After stabilization, the source was disconnected and the valve to the air bottle of known volume was opened. Once the temperature was stabilized, the final pressure readings were taken. Using Boyle's law, the porosity of the brass calibration sample that had a hole and whose porosity was known was calculated. This process was repeated on all core samples to determine the porosity of each core sample, as given in Table 5-2.

\section{Gas Flooding Experiments}

Nitrogen $\left(\mathrm{N}_{2}\right)$ was used as the flooding fluid. The pressures of the inlet and outlet BPR's were set to desired values. Flooding fluid was supplied through an injection pump, which was placed in the outer air bath. The fluid was injected at a constant flow rate. Fluid flowed into the core as soon as the injection pump pressure overcame the pressure at the inlet BPR. The pressure at the outlet BPR was set to be lower than the pressure at the inlet BPR (Fig. 5-1).

After the core was installed and the coreholder assembly was completed, required axial and radial stresses were applied using a high-pressure displacement pump. Sufficient time, usually several hours, was allowed for the coreholder assembly and core to reach the equilibrium temperature. Nitrogen was programmed to flow from the injection pump at staged flow rates changing from $25 \mathrm{cc} / \mathrm{hr}$ to $10000 \mathrm{cc} / \mathrm{hr}$, at an inlet BPR pressure of $2000 \mathrm{psi}$ and temperature of $80^{\circ} \mathrm{F}$. 
During each test, the temperatures, the stresses and the BPR pressures were kept unchanged. The gas was flooded through the sample at different flow rates. At each flow rate, flooding continued until the flow reached equilibrium, indicated by a stable pressure drop between the two ends of the sample. When flow equilibrium was reached, the pressures and temperatures at the inlet and outlet of the sample and at the injection pump were measured. The inlet BPR pressure was held constant to control the pressure of the accumulator to enable determination of the constant mass flow rate at any given setting. The pressure difference between the inlet and outlet of the sample was also measured. During the flooding process, the temperatures at the inner and outer air baths were monitored to for any deviations from the isothermal process.

Upon completion of one flow rate, the injection pump went to the next flow rate; pressures and temperatures were measured at equilibrium under this new flow rate. This process continued until all scheduled flow rates were completed. As each series of scheduled flow rates were finished, new axial and radial pressures were set for the next in-situ stress field, and a new flooding series started, with flow rates from low to high. When all the planned combinations of axial and radial stresses were completed, the temperature was then changed for a similar set of series at another temperature. As each cycle of tests under different combinations of in-situ stress fields and temperatures was finished, repeat tests were conducted at the initial experimental conditions of the cycle to check for hysteresis. Once the planned experiments on one rock sample were completed, another rock sample was installed; and another cycle of tests began.

Due to the capacity limit of the injection pump, the flooding gas had to be refilled from time to time. After each refill, new thermal equilibrium was required and gas flooding continued, repeating the last flow rate.

In total, 159 sets of experiments were conducted at different combinations of in-situ stress fields, temperature, outlet/pore pressures, and core samples as summarized in Table 5-3. 


\section{Results and Discussion}

\section{Theory and Formulas for Data Process}

Determination of non-Darcy Gas Flow Parameters Forchheimer's theory ${ }^{17}$ is used to calculate the non-Darcy flow parameters, i.e., the permeability, $\mathrm{k}$, and the non-Darcy flow coefficient, $\beta$. According to Green et al., ${ }^{18}$ Forchheimer's equation can be expressed as:

$\left(-\frac{d p}{d l}\right)=\frac{\mu}{k} v+\beta \rho v^{2}$

Using the PVT relationships of real gases, Eq. 5-1 can be changed into: ${ }^{19}$

$$
\frac{M\left(p_{1}{ }^{2}-p_{2}{ }^{2}\right)}{2 z R T \mu l\left(\frac{W}{A}\right)}=\frac{1}{k}+\beta\left(\frac{W}{\mu A}\right)
$$

In Eq. 5-2, $\mathrm{M}$ and $\mathrm{R}$ are gas constants; 1 and $\mathrm{A}$ are known from sample geometry; $p_{1}$ and $p_{2}$ are the measured pressures at the inlet and outlet of the core sample at temperature, $\mathrm{T}$ under mass flow rate, W. Gas compressibility factor, $\mathrm{z}$ and viscosity, $\mu$ are available from flash test results or from other resources, such as the PVT simulator, for the specific gas at the known average pressure and temperature. In this study, $\mathrm{z}$ and $\mu$ for nitrogen flooding were calculated using Calsep Inc.'s phase behavior simulator PVTsim ${ }^{\mathrm{TM}}$ with the Peng-Robinson Equation of State option. $^{20}$ The following correlations between $\mathrm{z}, \mu$ and pressure, $\mathrm{p}$, at temperatures of $10 \tilde{0}^{\circ} \mathrm{F}$, $150^{\circ} \mathrm{F}$ and $200^{\circ} \mathrm{F}$ were used: ${ }^{15}$

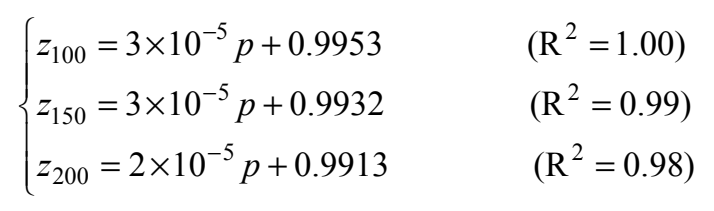

and

$$
\begin{cases}\mu_{100}=5 \times 10^{-5} p+0.5928 & \left(\mathrm{R}^{2}=1.00\right) \\ \mu_{150}=5 \times 10^{-5} p+0.5858 & \left(\mathrm{R}^{2}=1.00\right) \\ \mu_{200}=4 \times 10^{-5} p+0.5867 & \left(\mathrm{R}^{2}=1.00\right)\end{cases}
$$

Using Eqs. 5-3 and 5-4, the z-factor and the viscosity are calculated at each corresponding pressure and temperature. Values of viscosity where checked against experimental results ${ }^{21}$ and were found to be $2 \%$ to $4 \%$ high.

Now with $z$ and $\mu$ known, Eq. 5-2 has only two unknowns, $1 / \mathrm{k}$ and $\beta$, which is obviously similar 
to the following simple linear equation:

$$
Y=a X+b
$$

From Eq. 5-2, under constant temperature, T, the two unknowns, $1 / \mathrm{k}$ and $\beta$, can be determined from linear regression by the measured pressures at the inlet and outlet of the sample under two or more different flow rates.

Table 5-4 shows an example of the measured and calculated values for a test on Dakota sandstone under $100^{\circ} \mathrm{F}$. The calculation for the permeability, $\mathrm{k}$, and non-Darcy flow coefficient, $\square$, is shown in Fig. 5-2. Following the same procedure, permeability and non-Darcy flow coefficient of all the rest of the 159 experiments have been calculated, as shown in Table 5-5.

\section{Calculation of Stresses}

The stress sensitivity of the non-Darcy flow parameter results from two mechanisms: normal compaction (expansion) and shear deformation. Compaction is controlled by effective stresses; and deformation by shear stresses. ${ }^{22}$ Therefore, to investigate the stress-sensitivity of the nonDarcy flow parameter is to investigate the influences of these two stress components on the nonDarcy flow parameters.

Using the same equations as defined by Zeng, Grigg, and Ganda in a previous paper, ${ }^{14}$ the effective stress and the shear stress are calculated as:

$$
\left\{\begin{array}{l}
\sigma_{\text {eff }}=\frac{1}{3}\left(\sigma_{1 e f f}+\sigma_{2 e f f}+\sigma_{3 e f f}\right) \\
\tau=\frac{\sqrt{\left(\sigma_{1 e f f}-\sigma_{2 e f f}\right)^{2}+\left(\sigma_{2 e f f}-\sigma_{3 e f f}\right)^{2}+\left(\sigma_{3 e f f}-\sigma_{\text {leff }}\right)^{2}}}{3}
\end{array}\right.
$$

where $\sigma_{1 \text { eff }}, \sigma_{2 \text { eff }}$, and $\sigma_{3 \text { eff }}$ are the effective principal stresses, calculated as follows for the triaxial experiments in this study:

$$
\left\{\begin{array}{l}
\sigma_{\text {leff }}=\max \left\{\sigma_{a}-p_{c_{-} a v g}, \sigma_{r}-p_{c_{-} a v g}\right\} \\
\sigma_{2 e f f}=\sigma_{r}-p_{c_{-} a v g} \\
\sigma_{3 e f f}=\min \left\{\sigma_{a}-p_{c_{-} a v g}, \sigma_{r}-p_{c_{-} a v g}\right\}
\end{array}\right.
$$

where $\mathrm{p}_{\mathrm{c}_{-} \text {avg }}$ is the average core pressure that equals the average of the pore pressures correspond to the lowest and the highest flow rates in the same series of experiments. Values of the effective 
stress and the shear stress for each series of experiments were calculated and are summarized in Table 5-5.

\section{Stress-Sensitivity Analysis}

Based on the results shown in Table 5-5, stress-sensitivity of $\mathrm{k}$ and $\beta$ is analyzed in this section.

\section{Sensitivity to Effective Stress}

Using the results shown in Table 5-5, the sensitivity of non-Darcy flow parameters to effective normal was obtained as explained in the following sections.

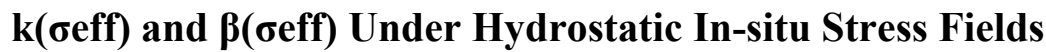

Some of the experiments were conducted under hydrostatic in-situ stress fields, as summarized in Table 5-5. Figures 5-3 and 5-4 show the influence of effective stress on permeability, $\mathrm{k}$, and nonDarcy flow coefficient, $\beta$, at three temperatures: $100^{\circ} \mathrm{F}, 150^{\circ} \mathrm{F}$ and $200^{\circ} \mathrm{F}$ in four different rocks.

It can be seen that permeability decreased in all four rocks, while the non-Darcy flow coefficient increased linearly with the effective stresses under all three temperatures. In addition, under the same effective stress, permeability decreased, while non-Darcy flow coefficient increased with temperature.

The above linear change of $\mathrm{k}$ and $\beta$ with $\sigma_{\text {eff }}$ are quantitatively correlated as follows:

(1) Dakota sandstone:

$\begin{cases}k_{100}=-4.48 \times 10^{-5} \sigma_{e f f}+3.47 & \left(\mathrm{R}^{2}=0.97\right) \\ k_{150}=-2.88 \times 10^{-5} \sigma_{e f f}+3.27 & \left(\mathrm{R}^{2}=0.99\right) \\ k_{200}=-3.26 \times 10^{-5} \sigma_{e f f}+3.21 & \left(\mathrm{R}^{2}=0.92\right)\end{cases}$
$\begin{cases}\beta_{100}=5.10 \times 10^{-3} \sigma_{e f f}+154.06 & \left(\mathrm{R}^{2}=0.95\right) \\ \beta_{150}=5.96 \times 10^{-3} \sigma_{e f f}+176.41 & \left(\mathrm{R}^{2}=0.95\right) \\ \beta_{200}=5.57 \times 10^{-3} \sigma_{e f f}+196.12 & \left(\mathrm{R}^{2}=0.99\right)\end{cases}$

(2) Low perm Indiana limestone 
$\begin{cases}k_{100}=-2.03 \times 10^{-4} \sigma_{e f f}+21.95 & \left(\mathrm{R}^{2}=0.75\right) \\ k_{150}=-2.22 \times 10^{-4} \sigma_{e f f}+19.80 & \left(\mathrm{R}^{2}=0.92\right) \\ k_{200}=-2.13 \times 10^{-4} \sigma_{e f f}+17.56 & \left(\mathrm{R}^{2}=0.96\right)\end{cases}$

$\begin{cases}\beta_{100}=5.39 \times 10^{-4} \sigma_{e f f}+34.93 & \left(\mathrm{R}^{2}=0.94\right) \\ \beta_{150}=7.29 \times 10^{-4} \sigma_{e f f}+39.76 & \left(\mathrm{R}^{2}=0.97\right) \\ \beta_{200}=6.14 \times 10^{-4} \sigma_{e f f}+43.54 & \left(\mathrm{R}^{2}=0.91\right)\end{cases}$

(3) Low perm Berea sandstone

$$
\begin{gathered}
\begin{cases}k_{100}=-1.99 \times 10^{-3} \sigma_{e f f}+216.77 & \left(\mathrm{R}^{2}=0.92\right) \\
k_{150}=-2.40 \times 10^{-3} \sigma_{\text {eff }}+199.25 & \left(\mathrm{R}^{2}=0.78\right) \\
k_{200}=-3.08 \times 10^{-3} \sigma_{\text {eff }}+180.25 & \left(\mathrm{R}^{2}=0.94\right)\end{cases} \\
\begin{cases}\beta_{100}=1.75 \times 10^{-5} \sigma_{e f f}+2.70 & \left(\mathrm{R}^{2}=0.43\right) \\
\beta_{150}=1.55 \times 10^{-5} \sigma_{e f f}+2.97 & \left(\mathrm{R}^{2}=0.28\right) \\
\beta_{200}=1.10 \times 10^{-5} \sigma_{\text {eff }}+3.29 & \left(\mathrm{R}^{2}=0.19\right)\end{cases}
\end{gathered}
$$

(4) High perm Berea sandstone

$$
\begin{aligned}
& \begin{cases}k_{100}=-3.05 \times 10^{-2} \sigma_{e f f}+1158.64 & \left(\mathrm{R}^{2}=0.78\right) \\
k_{150}=-1.91 \times 10^{-2} \sigma_{e f f}+826.13 & \left(\mathrm{R}^{2}=0.82\right) \\
k_{200}=-0.78 \times 10^{-2} \sigma_{e f f}+639.52 & \left(\mathrm{R}^{2}=0.20\right)\end{cases} \\
& \begin{cases}\beta_{100}=4.10 \times 10^{-5} \sigma_{e f f}+0.98 & \left(\mathrm{R}^{2}=0.95\right) \\
\beta_{150}=2.90 \times 10^{-5} \sigma_{e f f}+1.63 & \left(\mathrm{R}^{2}=0.99\right) \\
\beta_{200}=6.85 \times 10^{-5} \sigma_{e f f}+1.83 & \left(\mathrm{R}^{2}=0.33\right)\end{cases}
\end{aligned}
$$

From Eqs. 5-7, 5-9, 5-11 and 5-13, it can be seen that $\mathrm{k}-\sigma_{\text {eff }}$ correlations can be represented by the following general $\mathrm{k}\left(\sigma_{\text {eff }}\right)$ function:

$k=-s_{k} \sigma_{e f f}+k_{0}$

where $s_{k}$ is the sensitivity of permeability to effective stress, and $k_{0}$ is the nominal permeability corresponding to zero effective stress. Similarly, general $\beta\left(\sigma_{\text {eff }}\right)$ function can be expressed as:

$$
\beta=s_{\beta} \sigma_{e f f}+\beta_{0}
$$


where $s_{\beta}$ is the sensitivity of non-Darcy flow coefficient to effective stress, $\beta \beta_{0}$ is the nominal non-Darcy flow coefficient corresponding to zero effective stress.

\section{$k\left(\sigma_{\text {eff }}\right)$ and $\beta\left(\sigma_{\text {eff }}\right)$ under Differential In-Situ Stress Fields}

Under differential in-situ stress fields, the validity of the above results is very important because, as mentioned before, most reservoir formation rocks are under differential in-situ stress fields. To validate the above results under differential in-situ stress fields, experiments have been designed to measure non-Darcy flow parameters, $\mathrm{k}$ and $\beta$, at three temperatures, $100^{\circ} \mathrm{F}, 150^{\circ} \mathrm{F}$ and $200^{\circ} \mathrm{F}$. Figure $5-5$ shows the $\mathrm{k}-\sigma_{\text {eff }}$ and $\beta-\sigma_{\text {eff }}$ relations. Their quantitative correlations for Dakota sandstone are:

$$
\begin{cases}k_{100}=-3.67 \times 10^{-5} \sigma_{e f f}+3.37 & \left(\mathrm{R}^{2}=0.86\right) \\ k_{150}=-3.85 \times 10^{-5} \sigma_{e f f}+3.39 & \left(\mathrm{R}^{2}=0.74\right) \\ k_{200}=-3.61 \times 10^{-5} \sigma_{e f f}+3.25 & \left(\mathrm{R}^{2}=0.80\right)\end{cases}
$$

and

$$
\begin{cases}\beta_{100}=5.48 \times 10^{-3} \sigma_{e f f}+153.97 & \left(\mathrm{R}^{2}=0.90\right) \\ \beta_{150}=6.53 \times 10^{-3} \sigma_{e f f}+173.93 & \left(\mathrm{R}^{2}=0.88\right) \\ \beta_{200}=6.68 \times 10^{-3} \sigma_{e f f}+200.90 & \left(\mathrm{R}^{2}=0.92\right)\end{cases}
$$

Comparing Eq. 5-17 to its counterpart under hydrostatic in-situ stress fields, Eq. 5-7, it is adequate to say that $\mathrm{k}$ has similar correlations with $\sigma_{\mathrm{eff}}$ under both hydrostatic and differential insitu stress fields. The same conclusion is true to the $\beta-\sigma_{\text {eff }}$ correlation under these two in-situ stress fields.

\section{$k\left(\sigma_{\text {eff }}\right)$ and $\beta\left(\sigma_{\text {eff }}\right)$ under Different Reservoir Pressures.}

The previous experiments were all run under outlet BPR pressure of 500 psi. The effects of reservoir pressures on the above observations were investigated using experiments under different outlet BPR pressures. Figure 5-6 shows the comparison of experimental results under outlet pressures of $500 \mathrm{psi}$ and $1500 \mathrm{psi}$ at $100^{\circ} \mathrm{F}$. It can be seen that, under different outlet BPR 
pressures, i.e., different reservoir pressures, the linear trends for the change of $\mathrm{k}$ and $\beta$ against $\sigma_{\text {eff }}$ still hold true. Equations 5-19 and 5-20 show the quantitative correlations:

$\begin{cases}k_{500}=-2.62 \times 10^{-3} \sigma_{\text {eff }}+81.70 & \left(\mathrm{R}^{2}=0.68\right) \\ k_{1500}=-2.58 \times 10^{-3} \sigma_{e f f}+86.89 & \left(\mathrm{R}^{2}=0.78\right)\end{cases}$

and

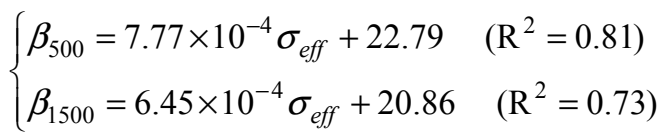

In addition, from Fig. 5-6, it is seen that, under the same effective stress, $\mathrm{k}$ increases while $\beta$ decreases with reservoir pressures. Comparing temperature influence on $\mathrm{k}$ and $\beta$ under the same effective stress, it is observed that the influence of reservoir pressure and temperature on $\mathrm{k}$ and $\beta$ are opposite.

\section{General Equations of Stress Sensitivity}

From Eqs. 5-7, 5-9, 5-11 and 5-13, it can be seen that the stress sensitivity $s_{k}$ increases with $\mathrm{k}_{0}$; a similar trend is found between $s_{\beta}$ and $\beta_{0}$ in Eqs. 5-8, 5-10, 5-12 and 5-14. Figure 5-7 shows the $\mathrm{k}_{0}-\mathrm{s}_{\mathrm{k}}$ curves and the $\beta_{0}-\mathrm{s}_{\beta}$ curves for different temperatures in different rocks. It is obvious that, regardless of the type of rock and testing temperature, distribution of all the $\left(\mathrm{k}_{0}, \mathrm{~s}_{\mathrm{k}}\right)$ points follow an orderly trend, as do the $\left(\beta_{0}, s_{\beta}\right)$ points. From these distributions, the general equations of stress sensitivity of non-Darcy flow parameters are obtained as follows:

$s_{k}=2 \times 10^{-8} k_{0}^{2}+5 \times 10^{-6} k_{0} \quad\left(\mathrm{R}^{2}=0.98\right)$

and

$s_{\beta}=3 \times 10^{-5} \beta_{0} \quad\left(\mathrm{R}^{2}=0.97\right)$

Using these two general equations, the stress sensitivity of a formation rock can be estimated when $\mathrm{k}_{0}$ and $\beta_{0}$ are known. Furthermore, with $\mathrm{s}_{\mathrm{k}}$ and $\mathrm{s}_{\beta}$ from Eqs. 5-21 and 5-22, change of the 
non-Darcy flow parameters due to change of reservoir pressure can be predicted using Eqs. 5-15 and 5-16.

\section{Sensitivity to Shear Stress}

$k(\tau)$ and $\beta(\tau)$ under Differential Temperatures. Previous work ${ }^{14}$ indicated that $k$ and $\beta$ are independent of shear stress at $100^{\circ} \mathrm{F}$. More experiments have been conducted under higher temperatures of $150^{\circ} \mathrm{F}$ and $200^{\circ} \mathrm{F}$. Figure 5-8 shows the $\tau-\mathrm{k}$ and $\tau-\beta$ curves under these three temperatures. Equations 5-23 and 5-24 show their correlations:

$$
\begin{cases}k_{100}=-8.06 \times 10^{-6} \tau+3.20 & \left(\mathrm{R}^{2}=0.01\right) \\ k_{150}=2.17 \times 10^{-5} \tau+3.15 & \left(\mathrm{R}^{2}=0.06\right) \\ k_{200}=1.05 \times 10^{-5} \tau+3.04 & \left(\mathrm{R}^{2}=0.02\right)\end{cases}
$$

and

$$
\begin{cases}\beta_{100}=-5.22 \times 10^{-4} \tau+182.18 & \left(\mathrm{R}^{2}=0.00\right) \\ \beta_{150}=3.61 \times 10^{-3} \tau+196.59 & \left(\mathrm{R}^{2}=0.02\right) \\ \beta_{200}=-3.01 \times 10^{-3} \tau+241.47 & \left(\mathrm{R}^{2}=0.04\right)\end{cases}
$$

The previous conclusion about the independence among $\mathrm{k}, \beta$ and $\tau$ is again confirmed here by more results. It is evidenced by the scattered distribution of $(\tau, \mathrm{k})$ points and $(\tau, \beta)$ points in Fig. $5-8$, and by the extremely low value of the coefficient of determination, $\mathrm{R}^{2}$, in Eqs. 5-23 and 524.

\section{$k(\tau)$ and $\beta(\tau)$ under Different Pore Pressures}

The independence of $\mathrm{k}, \beta$ on shear stress, $\tau$, is further examined with, and confirmed by, the results of experiments under pore pressures of 500 psi and 1500 psi. Figure 5-9 shows their graphic relationship, and Eqs. 5-25 and 5-26 the quantitative correlations.

$$
\left\{\begin{array}{l}
k_{500}=-3.86 \times 10^{-3} \tau+75.27 \quad\left(\mathrm{R}^{2}=0.34\right) \\
k_{1500}=-2.47 \times 10^{-3} \tau+78.65 \quad\left(\mathrm{R}^{2}=0.16\right)
\end{array}\right.
$$


and

$\left\{\begin{array}{l}\beta_{500}=1.18 \times 10^{-3} \tau+24.13 \quad\left(\mathrm{R}^{2}=0.22\right) \\ \beta_{1500}=2.30 \times 10^{-4} \tau+23.56 \quad\left(\mathrm{R}^{2}=0.03\right)\end{array}\right.$

\section{General Comments on Shear Stress Sensitivity}

The results of $\mathrm{k}(\tau)$ and $\beta(\tau)$ under different temperatures and different pore pressures in different rocks consistently confirm that non-Darcy flow parameters $\mathrm{k}$ and $\beta$ are independent of shear stresses. These results are consistent with previous observations. ${ }^{14}$

\section{Conclusions}

In summary, 159 series of high temperature, high pressure and high velocity gas flooding experiments have been conducted in five rocks to investigate the stress sensitivity of non-Darcy flow parameters. The tested rocks have petrophysical properties that represent many reservoir formations. The test conditions cover the majority of field in-situ stress fields, temperatures and gas production/injection flow rates. All the experiments gave consistent results which confirmed and extended previous conclusions observed in one single rock at a single temperature. Based on these experiments in this study, the following conclusions are derived:

1. Permeability, $\mathrm{k}$, decreases while non-Darcy flow coefficient, $\beta$, increases linearly with effective stresses.

2. General correlations, $\mathrm{k}\left(\sigma_{\text {eff }}\right)$ and $\beta\left(\sigma_{\text {eff }}\right)$ were derived in terms of stress sensitivity, $\mathrm{s}_{\mathrm{k}}$ and $\mathbf{s}_{\square}$, and nominal non-Darcy flow parameters, $\mathrm{k}_{0}$ and $\beta_{0}$. Influence of reservoir pressure change on $\mathrm{k}$ and $\beta$ can be predicted using these correlations.

3. Stress sensitivities, $s_{k}$ and $s_{\beta}$, of non-Darcy flow parameters, $k$ and $\beta$, are proportional to the nominal permeability, $\mathrm{k}_{0}$, and nominal non-Darcy flow coefficient, $\beta_{0}$, regardless of rock properties, in-situ stress fields, and temperatures. General stress sensitivity equations have been developed and can be used to estimate the stress sensitivities of non-Darcy flow parameters under given conditions of the reservoir. 
4. Under the same effective stress, permeability increases with reservoir pressures and decreases with reservoir temperatures; non-Darcy flow coefficient changes in an opposite manner under these two factors.

5. Shear stress does not have significant correlation with permeability and non-Darcy flow coefficient, indicating that $\mathrm{k}$ and $\beta$ are not sensitive to the change of shear stresses.

\section{Nomenclature}

$$
\begin{array}{ll}
\mathrm{a} & =\text { dummy variable } \\
\mathrm{A} & =\text { cross-sectional area of the sample, } \mathrm{cm}^{2} \\
\mathrm{~b} & =\text { dummy variable } \\
& =\beta \text { in figure legends } \\
\text {-dp/dl } & =\text { pressure gradient, atm } / \mathrm{cm} \\
\mathrm{k} & =\text { permeability, Darcy }(\mathrm{mD} \text { in correlations) } \\
k_{0} & =\text { nominal permeability at zero effective stress, } \mathrm{mD} \\
k_{100} & =\text { permeability at } 100^{\circ} \mathrm{F}, \mathrm{mD} \\
k_{150} & =\text { permeability at } 150^{\circ} \mathrm{F}, \mathrm{mD} \\
k_{200} & =\text { permeability at } 200^{\circ} \mathrm{F}, \mathrm{mD} \\
k_{500} & =\text { permeability at } 500 \mathrm{psi} \text { pore pressure, } \mathrm{mD} \\
k_{1500} & =\text { permeability at } 1500 \mathrm{psi} \text { pore pressure, } \mathrm{mD} \\
1 & =\text { sample length, cm } \\
\mathrm{M} & =\text { molecular weight, } \mathrm{g} / \mathrm{mole} \\
\mathrm{p}_{\mathrm{p}} & =\text { injection pump pressure, atm } \\
\mathrm{p}_{1} & =\text { pressure at core inlet, atm } \\
\mathrm{p}_{2} & =\text { pressure at core outlet, atm } \\
\mathrm{p}_{\mathrm{c} \_ \text {avg }} & =\text { average core pressure, } \mathrm{psi} \\
\mathrm{p}_{\mathrm{in}} & =\text { inlet BPR pressure, } \mathrm{psi} \\
\mathrm{p}_{\text {out }} & =\text { outlet BPR pressure, } \mathrm{psi} \\
\mathrm{Q}_{\mathrm{p}} & =\text { volumetric flow rate, cc/s } \\
\mathrm{R} & =\text { universal gas constant, } 82.06 \text { atm- } \mathrm{cm}{ }^{3} / \mathrm{g}-\mathrm{mole}-\mathrm{K} \\
\mathrm{R}^{2} & =\text { coefficient of determination } \\
s_{k} & =\text { stress-sensitivity of permeability, } \mathrm{mD} / \mathrm{psi} \\
s_{\square} & =\text { stress-sensitivity of non-Darcy flow coefficient, } 10^{6} / \mathrm{cm}-\mathrm{psi}
\end{array}
$$




$$
\begin{aligned}
& \mathrm{T}=\text { temperature, } \mathrm{K} \text { or } \mathrm{F} \\
& \mathrm{v}=\text { superficial velocity, } \mathrm{cm} / \mathrm{s} \\
& \mathrm{W}=\text { mass flow rate, } \mathrm{g} / \mathrm{s} \\
& \mathrm{X}=\text { dummy variable } \\
& \mathrm{Y} \quad=\text { dummy variable } \\
& \mathrm{z}=\text { gas compressibility factor } \\
& \mathrm{z}_{\mathrm{c}} \quad=\text { gas compressibility factor in the core } \\
& \mathrm{z}_{100}=\text { gas compressibility factor at } 100^{\circ} \mathrm{F} \\
& \mathrm{Z}_{150} \quad=\text { gas compressibility factor at } 150^{\circ} \mathrm{F} \\
& \mathrm{Z}_{200}=\text { gas compressibility factor at } 200^{\circ} \mathrm{F} \\
& \beta=\text { non-Darcy flow coefficient, atm- } \mathrm{s}^{2} / \mathrm{g}\left(=10^{6} 1 / \mathrm{cm}\right) \\
& \beta_{0} \quad=\text { nominal non-Darcy flow coefficient at zero effective stress, atm- } \mathrm{s}^{2} / \mathrm{g}\left(=10^{6} 1 / \mathrm{cm}\right) \\
& \beta_{100}=\text { non-Darcy flow coefficient at } 100^{\circ} \mathrm{F}, \mathrm{atm}-\mathrm{s}^{2} / \mathrm{g}\left(=10^{6} 1 / \mathrm{cm}\right) \\
& \beta_{150}=\text { non-Darcy flow coefficient at } 150^{\circ} \mathrm{F}, \text { atm- } \mathrm{s}^{2} / \mathrm{g}\left(=10^{6} 1 / \mathrm{cm}\right) \\
& \beta_{200}=\text { non-Darcy flow coefficient at } 200^{\circ} \mathrm{F}, \text { atm }-\mathrm{s}^{2} / \mathrm{g}\left(=10^{6} 1 / \mathrm{cm}\right) \\
& \beta_{500}=\text { non-Darcy flow coefficient at } 500 \mathrm{psi} \text { pore pressure, atm- } \mathrm{s}^{2} / \mathrm{g}\left(=10^{6} 1 / \mathrm{cm}\right) \\
& \beta_{1500}=\text { non-Darcy flow coefficient at } 1500 \mathrm{psi} \text { pore pressure, atm- } \mathrm{s}^{2} / \mathrm{g}\left(=10^{6} 1 / \mathrm{cm}\right) \\
& \mu \quad=\text { fluid viscosity, } \mathrm{cp} \\
& \mu_{10 \tilde{0}} \quad=\text { fluid viscosity at } 100^{\circ} \mathrm{F}, \mathrm{cp} \\
& \mu_{150} \quad=\text { fluid viscosity at } 150^{\circ} \mathrm{F}, \mathrm{cp} \\
& \mu_{20 \tilde{0}} \quad=\text { fluid viscosity at } 200^{\circ} \mathrm{F}, \mathrm{cp} \\
& \rho \quad=\text { fluid density, } \mathrm{g} / \mathrm{cm}^{3} \\
& \rho_{\mathrm{c}} \quad \quad \quad \text { fluid density in the sample, } \mathrm{g} / \mathrm{cm}^{3} \\
& \rho_{\mathrm{p}} \quad=\text { fluid density in the injection pump, } \mathrm{g} / \mathrm{cm}^{3} \\
& \sigma_{\text {leff, }}=\text { effective maximum principal stress, psi } \\
& \sigma_{2 \text { eff, }} \quad=\text { effective medium principal stress, psi } \\
& \sigma_{3 \text { eff }}=\text { effective minimum principal stress, psi } \\
& \sigma_{\mathrm{a}} \quad=\text { axial stress, } \mathrm{psi} \\
& \sigma_{\text {eff }} \quad=\text { average effective stress, } \mathrm{psi} \\
& \sigma_{\mathrm{r}} \quad=\text { radial stress, } \mathrm{psi} \\
& \tau \quad=\text { average shear stress, psi }
\end{aligned}
$$




\section{References}

1. Warpinski, N,R.: "Hydraulic Fracturing in Tight, Fissured Media," J. Pet. Tech., Feb. 1991, 146-152.

2. Schechter, D.S., McDonald, P., Sheffield, T., Baker, R.: "Reservoir Characterization and $\mathrm{CO}_{2}$ Pilot Design in the Naturally Fractured Spraberry Trend Area," Paper SPE 35469 presented at the SPE Permian Basin Oil and Gas Recovery Conference, Midland, 27-29 March 1996.

3. Teufel, L.E. and Farrell, H.E.: "Interrelationship between In-Situ Stresses, Natural Fractures, and Reservoir Permeability Anisotropy: A Case Study of the Ekofisk Field, North Sea," Proc. ISRM Conference on Fractured and Jointed Rock, Lake Tahoe, 1992.

4. Lorenz, J.C.: "Stress-Sensitive Reservoirs," J. Pet. Tech., Jan. 1999, 61-63.

5. Gray, D.H., Fatt, I. and Bergamini, G.: "The Effect of Stress on Permeability of Sandstone Cores," SPEJ, June 1963, 95-100.

6. Bai, M., Meng, F. Roegiers, J.-C., and Green, S.: "Improved Determination of StressDependent Permeability for Anisotropic Formations," Paper SPE/ISRM 78188 presented at the SPE / ISRM Rock Mechanics Conference, Irving, 20-23 October 2002.

7. Wattenbarger, R.A.: "Chapter 35 Well Performance Equations," Petroleum Engineering Handbook (Ed. Bradley), Society of Petroleum Engineers, Richardson, TX, USA, 1988.

8. Firoozabadi, A. and Katz, D.L.: "An Analysis of High-Velocity Gas Flow through Porous Media," J. Pet. Tech., Feb. 1979.

9. Grigg, R.B. and Hwang, M.K.: "High Velocity Gas Flow Effects in Porous Gas-Water System," Paper SPE 39978 presented at the 1998 SPE Gas Technology Symposium, Calgary, March 15-18.

10. Ewing, R. E., Lazarov, R.D., Lyons, S.L., Papavassiliou, D.V., Pasciak, J. and Qin, G.: "Numerical Well Model for Non-Darcy Flow through Isotropic Porous Media," Computational Geosciences, 1999, 185-204.

11. Chin, L.Y., Raghavan, R., Thomas, L.K.: "Fully Coupled Geomechanics and Fluid-Flow Analysis of Wells with Stress-Dependent Permeability," Paper SPE 58968 presented at the 1998 SPE International Conference and Exhibition, Beijing, Nov. 2-6.

12. Tiss, M. and Evans, R. D.: "Measurement and Correlation of Non-Darcy Flow Coefficient in Consolidated Porous Media,” J. Pet. Sci. Eng. 1989, 3, 19-33. 
13. Avila, C.E. and Evans, R.D.: "The Effect of Temperature and Overburden Pressure upon the non-Darcy Flow coefficient in Porous Media," Proc. 27 $7^{\text {th }}$ U.S. Symp. Rock Mech., Univ. Alabama, 1985, 623-634.

14. Zeng, Z., Grigg, R. and Ganda, S.: "Experimental Study of Overburden and Stress on NonDarcy Gas Flow in Dakota Sandstone," Paper SPE 84069 presented at 2003 SPE Annual Technical Conference and Exhibition, Denver, Oct. 5-8.

15. Grigg, R.B.: "Improving $\mathrm{CO}_{2}$ Efficiency for Recovering Oil in Heterogeneous Reservoirs," annual technical progress report, DOE Contract No. DE-FG26-01BC15364, (Oct. 2003)

16. Jarrell, P.M., Fox, C.E., Stein, M. H. and Webb, S.L.: Practical Aspects of $\mathrm{CO}_{2}$ Flooding, SPE Monograph Volume 22, Society of Petroleum Engineers, Richardson, TX, (2002).

17. Forchheimer, P.: “Wasserbewegung durch Boden,” Zeit. Ver. Deutsch. Ing., 45, 1901, 17811788.

18. Green, L., Jr. and Duwez, P.: "Fluid Flow Through Porous Metals," J. Appl. Mech., March 1951, 39-45.

19. Katz, D.L. Cornell, D., Kobayashi, R. Poettman, F.H., Vary, J.A., Elenbaas, J.R. and Weinaug, C.F.: Handbook of Natural Gas Engineering, McGraw-Hill Book Co., Inc., New York City, 1959.

20. PVTsim version 13, 2003, Calsep A/S, GI. Lundtoftevej 1C, DK-2800 Lyngby, Denmark.

21. Golubev, I.F.: Visocity of Gases and Gas Mixtures a Handbook, Moskva 1959, Translated from Russian by Kondor, R., 1970.

22. Jaeger, J.C. and Cook, N.G.W.: Fundamentals of Rock Mechanics, Third Edition, Chapman and Hall, London, 1979, 17-30. 
Table 5-1. Selected Experimental Conditions

\begin{tabular}{|l|l|}
\hline Parameter & Value \\
\hline Overburden pressure & $2000 \sim 10000 \mathrm{psi}$ \\
\hline Axial stress & $2000 \sim 10000 \mathrm{psi}$ \\
\hline Radial stress & $2000 \sim 10000 \mathrm{psi}$ \\
\hline Inlet BPR $\backslash$ Accumulator pressure & $2000 \sim 2500 \mathrm{psi}$ \\
\hline Outlet BPR pressure & $500 \sim 1500 \mathrm{psi}$ \\
\hline Temperature & $100 \sim 200 \mathrm{~F}$ \\
\hline Flow rate at pump $\left(80^{\circ} \mathrm{F}, 2000 \mathrm{psi}\right)$ & $25 \sim 10000 \mathrm{cc} /$ hour \\
\hline Superficial velocity in core & $4 \sim 6775 \mathrm{ft} /$ day \\
\hline Sample size & 1 -in diameter by 2-in length \\
\hline Sample permeability & $1 \sim 1000 \mathrm{mD}$ \\
\hline
\end{tabular}

Table 5-2. Sample Specifications

\begin{tabular}{|l|l|l|l|l|l|}
\hline Rock & Sample & $\begin{array}{l}\text { Diameter, } \\
\text { in }\end{array}$ & $\begin{array}{l}\text { Length, } \\
\text { in }\end{array}$ & Porosity & $\begin{array}{l}\text { Permeability, } \\
\mathrm{mD}\end{array}$ \\
\hline $\begin{array}{l}\text { Tight Dakota } \\
\text { sandstone }\end{array}$ & DSS201 & 1.00 & 2.00 & 0.14 & $<10$ \\
\hline $\begin{array}{l}\text { Low perm Indiana } \\
\text { limestone }\end{array}$ & IL301 & 0.99 & 2.07 & 0.15 & $10 \sim 50$ \\
\hline $\begin{array}{l}\text { High perm Indiana } \\
\text { limestone }\end{array}$ & IL302 & 0.99 & 2.04 & 0.27 & $50 \sim 100$ \\
\hline $\begin{array}{l}\text { Low perm Berea } \\
\text { sandstone }\end{array}$ & BSSL301 & 0.99 & 1.98 & 0.18 & $100 \sim 500$ \\
\hline $\begin{array}{l}\text { High perm Berea } \\
\text { sandstone }\end{array}$ & BSSH301 & 0.98 & 2.13 & 0.23 & $>500$ \\
\hline
\end{tabular}

Table 5-3. Summary of Test Conditions for Each Sample

\begin{tabular}{|l|l|l|l|l|l|}
\hline Sample & $\mathbf{T},{ }^{\boldsymbol{}} \mathbf{F}$ & Stress, $\mathbf{p s i}$ & $\mathbf{p}_{\text {in, }}, \mathbf{p s i}$ & $\mathbf{p}_{\text {out }}$ psi & Tests \\
\hline DSS201 & 100,150200 & $2000 \sim 10000$ & 2000 & 500 & 78 \\
\hline BSSL301 & 100,150200 & $2000 \sim 10000$ & 2000 & 500 & 15 \\
\hline BSSH301 & 100,150200 & $2000 \sim 10000$ & 2000 & 500 & 15 \\
\hline IL301 & $100,150,200$ & $2000 \sim 10000$ & 2000 & 500 & 15 \\
\hline IL302 & 100 & $2000 \sim 10000$ & 2500 & 1500 & 36 \\
\hline Total & \multicolumn{3}{|l}{} & 159 \\
\hline
\end{tabular}


Table 5-4. Example of Measured and Calculated Values

\begin{tabular}{|r|r|r|r|r|r|r|r|r|r|r|}
\hline $\mathrm{Q}_{\mathrm{p}}$ & $\mathrm{P}_{1}$ & $\mathrm{P}_{2}$ & $\mathrm{P}_{\mathrm{p}}$ & $\rho_{\mathrm{p}}$ & $\mathrm{w}$ & $\rho_{\mathrm{c}}$ & $\mathrm{z}_{\mathrm{c}}$ & $\mu_{\mathrm{c}}$ & $\mathrm{X}$ & $\mathrm{Y}$ \\
\hline $\mathrm{cc} / \mathrm{hr}$ & $\mathrm{psi}$ & $\mathrm{psi}$ & $\mathrm{Psi}$ & $\mathrm{g} / \mathrm{cm}^{3}$ & $\mathrm{~g} / \mathrm{hr}$ & $\mathrm{g} / \mathrm{cm}^{3}$ & & $\mathrm{cp}$ & $100 / \mathrm{cm}$ & $1 /$ Darcy \\
\hline $\mathbf{2 5}$ & 490 & 488 & 2004 & 0.1442 & 3.60 & 0.0364 & 1.0026 & 0.0186 & 0.01 & 293.55 \\
\hline $\mathbf{5 0}$ & 492 & 488 & 2003 & 0.1441 & 7.21 & 0.0365 & 1.0026 & 0.0186 & 0.02 & 292.99 \\
\hline $\mathbf{1 0 0}$ & 497 & 488 & 2001 & 0.1440 & 14.40 & 0.0367 & 1.0026 & 0.0186 & 0.04 & 287.47 \\
\hline $\mathbf{2 0 0}$ & 507 & 490 & 2002 & 0.1440 & 28.81 & 0.0372 & 1.0027 & 0.0186 & 0.08 & 295.86 \\
\hline $\mathbf{4 0 0}$ & 530 & 493 & 2003 & 0.1441 & 57.64 & 0.0381 & 1.0028 & 0.0186 & 0.17 & 318.16 \\
\hline $\mathbf{6 0 0}$ & 553 & 495 & 2004 & 0.1442 & 86.50 & 0.0390 & 1.0029 & 0.0186 & 0.25 & 341.15 \\
\hline $\mathbf{8 0 0}$ & 575 & 495 & 2004 & 0.1442 & 115.34 & 0.0398 & 1.0030 & 0.0186 & 0.34 & 361.51 \\
\hline $\mathbf{1 0 0 0}$ & 599 & 496 & 2004 & 0.1442 & 144.17 & 0.0408 & 1.0032 & 0.0187 & 0.42 & 381.22 \\
\hline $\mathbf{1 5 0 0}$ & 665 & 500 & 2004 & 0.1442 & 216.26 & 0.0434 & 1.0036 & 0.0187 & 0.63 & 431.15 \\
\hline $\mathbf{2 0 0 0}$ & 727 & 500 & 2004 & 0.1442 & 288.35 & 0.0457 & 1.0039 & 0.0187 & 0.84 & 468.45 \\
\hline $\mathbf{2 5 0 0}$ & 796 & 501 & 2003 & 0.1441 & 360.26 & 0.0482 & 1.0043 & 0.0188 & 1.05 & 513.75 \\
\hline $\mathbf{3 0 0 0}$ & 865 & 502 & 2002 & 0.1440 & 432.09 & 0.0508 & 1.0046 & 0.0188 & 1.26 & 554.11 \\
\hline $\mathbf{3 5 0 0}$ & 934 & 503 & 2002 & 0.1440 & 504.11 & 0.0534 & 1.0050 & 0.0189 & 1.46 & 591.29 \\
\hline $\mathbf{4 0 0 0}$ & 1003 & 503 & 2001 & 0.1440 & 575.83 & 0.0560 & 1.0054 & 0.0189 & 1.67 & 627.77 \\
\hline $\mathbf{4 5 0 0}$ & 1072 & 504 & 2002 & 0.1440 & 648.14 & 0.0585 & 1.0058 & 0.0189 & 1.88 & 661.36 \\
\hline $\mathbf{5 0 0 0}$ & 1139 & 504 & 2001 & 0.1440 & 719.79 & 0.0610 & 1.0061 & 0.0190 & 2.08 & 692.39 \\
\hline $\mathbf{6 0 0 0}$ & 1276 & 505 & 2002 & 0.1440 & 864.18 & 0.0661 & 1.0068 & 0.0191 & 2.48 & 755.26 \\
\hline $\mathbf{7 0 0 0}$ & 1411 & 506 & 2002 & 0.1440 & 1008.21 & 0.0711 & 1.0076 & 0.0191 & 2.89 & 813.91 \\
\hline $\mathbf{8 0 0 0}$ & 1546 & 506 & 2001 & 0.1440 & 1151.67 & 0.0760 & 1.0083 & 0.0192 & 3.28 & 872.25 \\
\hline $\mathbf{9 0 0 0}$ & 1678 & 507 & 2001 & 0.1440 & 1295.63 & 0.0809 & 1.0090 & 0.0193 & 3.68 & 925.17 \\
\hline $\mathbf{1 0 0 0 0}$ & 1808 & 507 & 2002 & 0.1440 & 1440.31 & 0.0856 & 1.0097 & 0.0194 & 4.07 & 975.13 \\
\hline
\end{tabular}

Table 5-5. Summary of $k$ and $\beta$ of All the Tests

\begin{tabular}{|r|c|c|c|c|c|c|c|c|c|c|}
\hline Test & Core & $\mathbf{T}$ & $\mathbf{p}_{\text {in }}$ & $\mathbf{p}_{\text {out }}$ & $\boldsymbol{\sigma}_{\mathbf{a}}$ & $\boldsymbol{\sigma}_{\mathbf{r}}$ & $\boldsymbol{\sigma}_{\text {eff }}$ & $\boldsymbol{\tau}$ & $\mathbf{k}$ & $\boldsymbol{\beta}$ \\
\hline & & ${ }^{\circ} \mathbf{F}$ & $\mathbf{p s i}$ & $\mathbf{p s i}$ & $\mathbf{p s i}$ & $\mathbf{p s i}$ & $\mathbf{p s i}$ & $\mathbf{p s i}$ & $\mathbf{m D}$ & $\mathbf{1 0} / \mathbf{c m}$ \\
\hline 1 & DSS201 & 100 & 2000 & 500 & 2000 & 2000 & 1192.99 & 0.00 & 3.44 & 155.51 \\
\hline 2 & DSS201 & 100 & 2000 & 500 & 3000 & 3000 & 2179.71 & 0.00 & 3.36 & 165.13 \\
\hline 3 & DSS201 & 100 & 2000 & 500 & 4000 & 4000 & 3176.70 & 0.00 & 3.29 & 175.70 \\
\hline 4 & DSS201 & 100 & 2000 & 500 & 5000 & 5000 & 4177.93 & 0.00 & 3.27 & 175.84 \\
\hline 5 & DSS201 & 100 & 2000 & 500 & 6000 & 6000 & 5167.93 & 0.00 & 3.26 & 183.14 \\
\hline 6 & DSS201 & 100 & 2000 & 500 & 7000 & 7000 & 6163.67 & 0.00 & 3.16 & 189.36 \\
\hline 7 & DSS201 & 100 & 2000 & 500 & 8000 & 8000 & 7159.66 & 0.00 & 3.13 & 192.59 \\
\hline 8 & DSS201 & 100 & 2000 & 500 & 9000 & 9000 & 8161.65 & 0.00 & 3.11 & 194.43 \\
\hline 9 & DSS201 & 100 & 2000 & 500 & 10000 & 10000 & 9161.14 & 0.00 & 3.07 & 195.94 \\
\hline & & & & & & & & & & \\
\hline 10 & DSS201 & 100 & 2000 & 500 & 2000 & 3000 & 1846.63 & 471.40 & 3.29 & 164.26 \\
\hline 11 & DSS201 & 100 & 2000 & 500 & 2000 & 4000 & 2522.53 & 942.81 & 3.29 & 170.99 \\
\hline 12 & DSS201 & 100 & 2000 & 500 & 2000 & 5000 & 3177.47 & 1414.21 & 3.22 & 175.20 \\
\hline 13 & DSS201 & 100 & 2000 & 500 & 2000 & 6000 & 3851.85 & 1885.62 & 3.24 & 176.03 \\
\hline
\end{tabular}




\begin{tabular}{|c|c|c|c|c|c|c|c|c|c|c|}
\hline Test & Core & $\mathbf{T}$ & $p_{\text {in }}$ & $p_{\text {out }}$ & $\sigma_{a}$ & $\sigma_{\mathrm{r}}$ & $\sigma_{\text {eff }}$ & $\tau$ & $\mathbf{k}$ & $\beta$ \\
\hline & & ${ }^{\circ} \mathbf{F}$ & psi & psi & psi & psi & psi & psi & $\mathrm{mD}$ & $10 \% / \mathrm{cm}$ \\
\hline 14 & DSS201 & 100 & 2000 & 500 & 2000 & 7000 & 4505.52 & 2357.02 & 3.12 & 179.43 \\
\hline 15 & DSS201 & 100 & 2000 & 500 & 2000 & 8000 & 5181.42 & 2828.43 & 3.22 & 180.63 \\
\hline 16 & DSS201 & 100 & 2000 & 500 & 2000 & 9000 & 5843.58 & 3299.83 & 3.15 & 184.38 \\
\hline 17 & DSS201 & 100 & 2000 & 500 & 2000 & 10000 & 6506.74 & 3771.24 & 3.11 & 186.95 \\
\hline 18 & DSS201 & 100 & 2000 & 500 & 4000 & 2000 & 1873.10 & 942.81 & 3.33 & 162.91 \\
\hline 19 & DSS201 & 100 & 2000 & 500 & 4000 & 6000 & 4514.75 & 942.81 & 3.19 & 184.41 \\
\hline 20 & DSS201 & 100 & 2000 & 500 & 4000 & 8000 & 5849.32 & 1885.62 & 3.17 & 186.90 \\
\hline 21 & DSS201 & 100 & 2000 & 500 & 4000 & 10000 & 7176.42 & 2828.43 & 3.14 & 192.61 \\
\hline 22 & DSS201 & 100 & 2000 & 500 & 6000 & 2000 & 2549.50 & 1885.62 & 3.30 & 163.74 \\
\hline 23 & DSS201 & 100 & 2000 & 500 & 6000 & 4000 & 3862.32 & 942.81 & 3.21 & 178.38 \\
\hline 24 & DSS201 & 100 & 2000 & 500 & 6000 & 8000 & 6511.23 & 942.81 & 3.10 & 191.01 \\
\hline 25 & DSS201 & 100 & 2000 & 500 & 6000 & 10000 & 7848.30 & 1885.62 & 3.10 & 192.78 \\
\hline 26 & DSS201 & 100 & 2000 & 500 & 8000 & 2000 & 3216.41 & 2828.43 & 3.29 & 166.46 \\
\hline 27 & DSS201 & 100 & 2000 & 500 & 8000 & 4000 & 4529.46 & 1885.62 & 3.20 & 180.79 \\
\hline 28 & DSS201 & 100 & 2000 & 500 & 8000 & 6000 & 5862.79 & 942.81 & 3.17 & 189.90 \\
\hline 29 & DSS201 & 100 & 2000 & 500 & 8000 & 10000 & 8521.19 & 942.81 & 3.08 & 199.46 \\
\hline 30 & DSS201 & 100 & 2000 & 500 & 10000 & 2000 & 3892.55 & 3771.24 & 3.24 & 165.67 \\
\hline 31 & DSS201 & 100 & 2000 & 500 & 10000 & 4000 & 5205.63 & 2828.43 & 3.16 & 185.53 \\
\hline 32 & DSS201 & 100 & 2000 & 500 & 10000 & 6000 & 6530.95 & 1885.62 & 3.12 & 192.32 \\
\hline 33 & DSS201 & 100 & 2000 & 500 & 10000 & 8000 & 7856.27 & 942.81 & 3.07 & 198.00 \\
\hline 34 & DSS201 & 150 & 2000 & 500 & 2000 & 2000 & 1179.01 & 0.00 & 3.25 & 177.87 \\
\hline 35 & DSS201 & 150 & 2000 & 500 & 3000 & 3000 & & 0.00 & 3.20 & 186.94 \\
\hline 36 & DSS201 & 150 & 2000 & 500 & 4000 & 4000 & 3147.99 & 0.00 & 3.18 & 197.98 \\
\hline 37 & DSS201 & 150 & 2000 & 500 & 5000 & 5000 & 4142.98 & 0.00 & 3.16 & 206.69 \\
\hline 38 & DSS201 & 150 & 2000 & 500 & 6000 & 6000 & 5140.98 & 0.00 & 3.13 & 210.74 \\
\hline 39 & DSS201 & 150 & 2000 & 500 & 7000 & 7000 & 6137.97 & 0.00 & 3.10 & 213.03 \\
\hline 40 & DSS201 & 150 & 2000 & 500 & 8000 & 8000 & 7132.96 & 0.00 & 3.07 & 220.29 \\
\hline 41 & DSS201 & 150 & 2000 & 500 & 9000 & 9000 & 8129.21 & 0.00 & 3.04 & 223.38 \\
\hline 42 & DSS201 & 150 & 2000 & 500 & 10000 & 10000 & 9126.20 & 0.00 & 3.01 & 226.72 \\
\hline 43 & DSS201 & 150 & 2000 & 500 & 2000 & 4000 & 2487.57 & 942.81 & 3.20 & 190.74 \\
\hline 44 & DSS201 & 150 & 2000 & 500 & 2000 & 6000 & 3815.65 & 1885.62 & 3.20 & 204.47 \\
\hline 45 & DSS201 & 150 & 2000 & 500 & 2000 & 8000 & 5141.36 & 2828.43 & 3.19 & 210.03 \\
\hline 46 & DSS201 & 150 & 2000 & 500 & 2000 & 10000 & 6469.07 & 3771.24 & 3.16 & 217.99 \\
\hline 47 & DSS201 & 150 & 2000 & 500 & 4000 & 2000 & 1830.91 & 942.81 & 3.26 & 103.28 \\
\hline 48 & DSS201 & 150 & 2000 & 500 & 4000 & 6000 & 4479.57 & 942.81 & 3.17 & 206.14 \\
\hline 49 & DSS201 & 150 & 2000 & 500 & 4000 & 8000 & 5809.65 & 1885.62 & 3.15 & 209.80 \\
\hline 50 & DSS201 & 150 & 2000 & 500 & 4000 & 10000 & 7137.23 & 2828.43 & 3.12 & 216.22 \\
\hline 51 & DSS201 & 150 & 2000 & 500 & 6000 & 2000 & 2494.86 & 1885.62 & 3.34 & 191.32 \\
\hline 52 & DSS201 & 150 & 2000 & 500 & 6000 & 4000 & 3822.69 & 942.81 & 3.27 & 197.28 \\
\hline 53 & DSS201 & 150 & 2000 & 500 & 6000 & 8000 & 6481.84 & 942.81 & 3.21 & 205.36 \\
\hline 54 & DSS201 & 150 & 2000 & 500 & 6000 & 10000 & 7799.40 & 1885.62 & 3.07 & 222.68 \\
\hline
\end{tabular}




\begin{tabular}{|c|c|c|c|c|c|c|c|c|c|c|}
\hline Test & Core & $\mathbf{T}$ & $p_{\text {in }}$ & $p_{\text {out }}$ & $\sigma_{a}$ & $\sigma_{\mathrm{r}}$ & $\sigma_{\text {eff }}$ & $\tau$ & $\mathbf{k}$ & $\beta$ \\
\hline & & ${ }^{\circ} \mathbf{F}$ & psi & psi & psi & psi & psi & psi & $\mathrm{mD}$ & $10 \% / \mathrm{cm}$ \\
\hline 55 & DSS201 & 150 & 2000 & 500 & 8000 & 2000 & 3160.53 & 2828.43 & 3.32 & 192.60 \\
\hline 56 & DSS201 & 150 & 2000 & 500 & 8000 & 4000 & 4484.10 & 1885.62 & 3.25 & 202.90 \\
\hline 57 & DSS201 & 150 & 2000 & 500 & 8000 & 6000 & 5807.41 & 942.81 & 3.16 & 215.15 \\
\hline & & & & & & & & & & \\
\hline 58 & DSS201 & 150 & 2000 & 500 & 8000 & 10000 & 8461.80 & 942.81 & 3.04 & 228.47 \\
\hline 59 & DSS201 & 150 & 2000 & 500 & 10000 & 2000 & 3826.44 & 3771.24 & 3.29 & 192.82 \\
\hline 60 & DSS201 & 150 & 2000 & 500 & 10000 & 4000 & 5148.01 & 2828.43 & 3.22 & 205.83 \\
\hline 61 & DSS201 & 150 & 2000 & 500 & 10000 & 6000 & 6467.82 & 1885.62 & 3.13 & 222.93 \\
\hline 62 & DSS201 & 150 & 2000 & 500 & 10000 & 8000 & 7793.12 & 942.81 & 3.03 & 231.87 \\
\hline 63 & DSS201 & 200 & 2000 & 500 & 2000 & 2000 & 986.25 & 0.00 & 3.21 & 202.25 \\
\hline 64 & DSS201 & 200 & 2000 & 500 & 4000 & 4000 & 3103.75 & 0.00 & 3.07 & 210.77 \\
\hline 65 & DSS201 & 200 & 2000 & 500 & 6000 & 6000 & 5093.75 & 0.00 & 3.02 & 227.09 \\
\hline 66 & DSS201 & 200 & 2000 & 500 & 10000 & 10000 & 9079.25 & 0.00 & 2.93 & 245.98 \\
\hline 67 & DSS201 & 200 & 2000 & 500 & 2000 & 6000 & 3752.92 & 1885.62 & 3.05 & 221.65 \\
\hline 68 & DSS201 & 200 & 2000 & 500 & 2000 & 10000 & 6413.33 & 3771.24 & 2.95 & 237.85 \\
\hline 69 & DSS201 & 200 & 2000 & 500 & 4000 & 2000 & 1755.92 & 942.81 & 3.18 & 213.11 \\
\hline 70 & DSS201 & 200 & 2000 & 500 & 4000 & 6000 & 4417.33 & 942.81 & 3.08 & 234.84 \\
\hline 71 & DSS201 & 200 & 2000 & 500 & 4000 & 10000 & 7073.00 & 2828.43 & 3.02 & 244.20 \\
\hline 72 & DSS201 & 200 & 2000 & 500 & 6000 & 2000 & 2425.83 & 1885.62 & 3.19 & 217.75 \\
\hline 73 & DSS201 & 200 & 2000 & 500 & 6000 & 10000 & 7738.42 & 1885.62 & 3.02 & 253.41 \\
\hline 74 & DSS201 & 200 & 2000 & 500 & 8000 & 2000 & 3086.00 & 2828.43 & 3.19 & 227.30 \\
\hline 75 & DSS201 & 200 & 2000 & 500 & 8000 & 6000 & 5750.42 & 942.81 & 2.99 & 234.14 \\
\hline 76 & DSS201 & 200 & 2000 & 500 & 8000 & 10000 & 8395.08 & 942.81 & 2.96 & 258.16 \\
\hline 77 & DSS201 & 200 & 2000 & 500 & 10000 & 2000 & 3750.67 & 3771.24 & 3.14 & 223.53 \\
\hline 78 & DSS201 & 200 & 2000 & 500 & 10000 & 8000 & 7733.42 & 942.81 & 2.97 & 260.76 \\
\hline 79 & BSSL301 & 100 & 2000 & 500 & 2000 & 2000 & 1450.40 & 0.00 & 215.55 & 2.80 \\
\hline 80 & BSSL301 & 100 & 2000 & 500 & 4000 & 4000 & 3451.95 & 0.00 & 207.20 & 2.67 \\
\hline 81 & BSSL301 & 100 & 2000 & 500 & 6000 & 6000 & 5448.35 & 0.00 & 207.23 & 2.76 \\
\hline 82 & BSSL301 & 100 & 2000 & 500 & 8000 & 8000 & 7456.18 & 0.00 & 200.80 & 2.86 \\
\hline 83 & BSSL301 & 100 & 2000 & 500 & 10000 & 10000 & 9452.85 & 0.00 & 198.85 & 2.88 \\
\hline 84 & BSSL301 & 150 & 2000 & 500 & 2000 & 2000 & 1448.80 & 0.00 & 196.49 & 3.02 \\
\hline 85 & BSSL301 & 150 & 2000 & 500 & 4000 & 4000 & 3451.28 & 0.00 & 185.89 & 2.95 \\
\hline 86 & BSSL301 & 150 & 2000 & 500 & 6000 & 6000 & 5448.82 & 0.00 & 191.61 & 3.03 \\
\hline 87 & BSSL301 & 150 & 2000 & 500 & 8000 & 8000 & 7448.53 & 0.00 & 183.03 & 3.20 \\
\hline 88 & BSSL301 & 150 & 2000 & 500 & 10000 & 10000 & 9446.16 & 0.00 & 173.97 & 3.05 \\
\hline 89 & BSSL301 & 200 & 2000 & 500 & 2000 & 2000 & 1419.93 & 0.00 & 176.43 & 3.31 \\
\hline 90 & BSSL301 & 200 & 2000 & 500 & 4000 & 4000 & 3414.32 & 0.00 & 171.24 & 3.29 \\
\hline 91 & BSSL301 & 200 & 2000 & 500 & 6000 & 6000 & 5414.09 & 0.00 & 161.77 & 3.33 \\
\hline
\end{tabular}




\begin{tabular}{|c|c|c|c|c|c|c|c|c|c|c|}
\hline Test & Core & $\mathbf{T}$ & $\mathbf{p}_{\text {in }}$ & pout & $\sigma_{a}$ & $\sigma_{\mathbf{r}}$ & $\sigma_{\text {eff }}$ & $\tau$ & $\mathbf{k}$ & $\beta$ \\
\hline & & ${ }^{\circ} \mathbf{F}$ & psi & psi & psi & psi & psi & psi & mD & $10^{6} / \mathrm{cm}$ \\
\hline 92 & BSSL301 & 200 & 2000 & 500 & 8000 & 8000 & 7408.27 & 0.00 & 154.36 & 3.49 \\
\hline 93 & BSSL301 & 200 & 2000 & 500 & 10000 & 10000 & 9403.93 & 0.00 & 154.15 & 3.32 \\
\hline 94 & BSSH301 & 100 & 2000 & 500 & 2000 & 2000 & 1463.25 & 0.00 & 1079.45 & 1.02 \\
\hline 95 & BSSH301 & 100 & 2000 & 500 & 4000 & 4000 & 3463.09 & 0.00 & 1133.79 & 1.12 \\
\hline 96 & BSSH301 & 100 & 2000 & 500 & 6000 & 6000 & 5462.74 & 0.00 & 943.40 & 1.25 \\
\hline 97 & BSSH301 & 100 & 2000 & 500 & 8000 & 8000 & 7463.08 & 0.00 & 924.13 & 1.30 \\
\hline 98 & BSSH301 & 100 & 2000 & 500 & 10000 & 10000 & 9462.88 & 0.00 & 879.28 & 1.34 \\
\hline & & & & & & & & & & \\
\hline 99 & BSSH301 & 150 & 2000 & 500 & 2000 & 2000 & 1450.81 & 0.00 & 834.10 & 1.67 \\
\hline 100 & BSSH301 & 150 & 2000 & 500 & 4000 & 4000 & 3449.35 & 0.00 & 726.90 & 1.73 \\
\hline 101 & BSSH301 & 150 & 2000 & 500 & 6000 & 6000 & 5448.88 & 0.00 & 702.25 & 1.78 \\
\hline 102 & BSSH301 & 150 & 2000 & 500 & 8000 & 8000 & 7448.26 & 0.00 & 679.86 & 1.83 \\
\hline 103 & BSSH301 & 150 & 2000 & 500 & 10000 & 10000 & 9447.50 & 0.00 & 666.40 & 1.91 \\
\hline 104 & BSSH301 & 200 & 2000 & 500 & 2000 & 2000 & 1454.05 & 0.00 & 607.94 & 2.27 \\
\hline 105 & BSSH301 & 200 & 2000 & 500 & 4000 & 4000 & 3452.77 & 0.00 & 682.31 & 1.57 \\
\hline 106 & BSSH301 & 200 & 2000 & 500 & 6000 & 6000 & 5455.38 & 0.00 & 529.38 & 2.27 \\
\hline 107 & BSSH301 & 200 & 2000 & 500 & 8000 & 8000 & 7455.55 & 0.00 & 586.89 & 2.38 \\
\hline 108 & BSSH301 & 200 & 2000 & 500 & 10000 & 10000 & 9454.63 & 0.00 & 577.27 & 2.55 \\
\hline 109 & IL301 & 100 & 2000 & 500 & 2000 & 2000 & 1342.47 & 0.00 & 22.14 & 36.00 \\
\hline 110 & IL301 & 100 & 2000 & 500 & 4000 & 4000 & 3337.40 & 0.00 & 20.82 & 36.61 \\
\hline 111 & IL301 & 100 & 2000 & 500 & 6000 & 6000 & 5333.93 & 0.00 & 20.66 & 37.55 \\
\hline 112 & IL301 & 100 & 2000 & 500 & 8000 & 8000 & 7332.42 & 0.00 & 20.34 & 38.38 \\
\hline 113 & IL301 & 100 & 2000 & 500 & 10000 & 10000 & 9328.20 & 0.00 & 20.35 & 40.50 \\
\hline 114 & IL301 & 150 & 2000 & 500 & 2000 & 2000 & 1376.93 & 0.00 & 19.71 & 40.84 \\
\hline 115 & IL301 & 150 & 2000 & 500 & 4000 & 4000 & 3372.43 & 0.00 & 18.93 & 42.45 \\
\hline 116 & IL301 & 150 & 2000 & 500 & 6000 & 6000 & 5369.91 & 0.00 & 18.36 & 43.44 \\
\hline 117 & IL301 & 150 & 2000 & 500 & 8000 & 8000 & 7367.16 & 0.00 & 18.11 & 44.59 \\
\hline 118 & IL301 & 150 & 2000 & 500 & 10000 & 10000 & 9344.64 & 0.00 & 17.91 & 47.04 \\
\hline 119 & IL301 & 200 & 2000 & 500 & 2000 & 2000 & 1333.37 & 0.00 & 17.20 & 43.74 \\
\hline 120 & IL301 & 200 & 2000 & 500 & 4000 & 4000 & 3347.38 & 0.00 & 16.81 & 45.80 \\
\hline 121 & IL301 & 200 & 2000 & 500 & 6000 & 6000 & 5345.40 & 0.00 & 16.58 & 47.74 \\
\hline 122 & IL301 & 200 & 2000 & 500 & 8000 & 8000 & 7317.38 & 0.00 & 16.13 & 48.09 \\
\hline 123 & IL301 & 200 & 2000 & 500 & 10000 & 10000 & 9314.62 & 0.00 & 15.42 & 48.71 \\
\hline 124 & IL302 & 100 & 2000 & 500 & 1500 & 2000 & 1239.49 & 235.70 & 74.84 & 25.07 \\
\hline 125 & IL302 & 100 & 2000 & 500 & 1000 & 2000 & 1073.57 & 471.40 & 74.23 & 24.26 \\
\hline 126 & IL302 & 100 & 2000 & 500 & 1000 & 4000 & 2406.40 & 1414.21 & 75.52 & 24.31 \\
\hline
\end{tabular}




\begin{tabular}{|c|c|c|c|c|c|c|c|c|c|c|}
\hline Test & Core & $\mathbf{T}$ & $p_{\text {in }}$ & pout $_{\text {out }}$ & $\sigma_{a}$ & $\sigma_{\mathrm{r}}$ & $\sigma_{\text {eff }}$ & $\tau$ & $\mathbf{k}$ & $\beta$ \\
\hline & & ${ }^{\circ} \mathbf{F}$ & psi & psi & psi & psi & psi & psi & $\mathrm{mD}$ & $10 \% / \mathrm{cm}$ \\
\hline 127 & IL302 & 100 & 2000 & 500 & 1000 & 6000 & 3740.25 & 2357.02 & 78.22 & 24.41 \\
\hline 128 & IL302 & 100 & 2000 & 500 & 1000 & 8000 & 5039.09 & 3299.83 & 66.91 & 26.57 \\
\hline 129 & IL302 & 100 & 2000 & 500 & 1000 & 10000 & 6205.66 & 4242.64 & 93.91 & 42.14 \\
\hline 130 & IL302 & 100 & 2000 & 500 & 2000 & 4000 & 2737.64 & 942.81 & 72.75 & 25.42 \\
\hline & & & & & & & & & & \\
\hline 131 & IL302 & 100 & 2000 & 500 & 2000 & 6000 & 4073.85 & 1885.62 & 78.25 & 24.67 \\
\hline 132 & IL302 & 100 & 2000 & 500 & 2000 & 8000 & 5373.24 & 2828.43 & 62.59 & 25.52 \\
\hline 133 & IL302 & 100 & 2000 & 500 & 2000 & 10000 & 6699.44 & 3771.24 & 56.23 & 28.82 \\
\hline 134 & IL302 & 100 & 2000 & 500 & 3500 & 4000 & 3236.75 & 235.70 & 75.70 & 25.89 \\
\hline 135 & IL302 & 100 & 2000 & 500 & 3000 & 6000 & 4403.56 & 1414.21 & 73.02 & 25.74 \\
\hline 136 & IL302 & 100 & 2000 & 500 & 3000 & 8000 & 5708.25 & 2357.02 & 69.16 & 26.76 \\
\hline 137 & IL302 & 100 & 2000 & 500 & 3000 & 10000 & 7033.33 & 3299.83 & 61.20 & 29.12 \\
\hline 138 & IL302 & 100 & 2000 & 500 & 4000 & 6000 & 4732.64 & 942.81 & 62.60 & 18.48 \\
\hline 139 & IL302 & 100 & 2000 & 500 & 4000 & 8000 & 6040.58 & 1885.62 & 68.01 & 27.16 \\
\hline 140 & IL302 & 100 & 2000 & 500 & 5000 & 10000 & 7701.24 & 2357.02 & 59.96 & 28.93 \\
\hline 141 & IL302 & 100 & 2000 & 500 & 5000 & 8000 & 6374.41 & 1414.21 & 67.34 & 27.30 \\
\hline 142 & IL302 & 100 & 2000 & 500 & 5000 & 10000 & 7711.49 & 2357.02 & 61.64 & 29.65 \\
\hline 143 & IL302 & 100 & 2000 & 500 & 6000 & 8000 & 6706.01 & 942.81 & 68.01 & 27.76 \\
\hline 144 & IL302 & 100 & 2000 & 500 & 6000 & 10000 & 8044.58 & 1885.62 & 58.89 & 29.67 \\
\hline 145 & IL302 & 100 & 2500 & 1500 & 2500 & 6000 & 3253.10 & 1649.92 & 76.12 & 21.93 \\
\hline 146 & IL302 & 100 & 2500 & 1500 & 2000 & 6000 & 3086.68 & 1885.62 & 81.49 & 22.18 \\
\hline 147 & IL302 & 100 & 2500 & 1500 & 2000 & 8000 & 4406.71 & 2828.43 & 63.67 & 24.00 \\
\hline 148 & IL302 & 100 & 2500 & 1500 & 2000 & 10000 & 5742.51 & 3771.24 & 72.29 & 24.52 \\
\hline 149 & IL302 & 100 & 2500 & 1500 & 2000 & 6000 & 3085.03 & 1885.62 & 78.13 & 23.32 \\
\hline 150 & IL302 & 100 & 2500 & 1500 & 2000 & 6000 & 3089.10 & 1885.62 & 79.42 & 22.73 \\
\hline 151 & IL302 & 100 & 2000 & 1500 & 3000 & 8000 & 4743.67 & 2357.02 & 72.58 & 24.51 \\
\hline 152 & IL302 & 100 & 2500 & 1500 & 3000 & 10000 & 6077.75 & 3299.83 & 71.08 & 24.66 \\
\hline 153 & IL302 & 100 & 2500 & 1500 & 4000 & 6000 & 3755.07 & 942.81 & 81.21 & 23.66 \\
\hline 154 & IL302 & 100 & 2500 & 1500 & 4000 & 8000 & 5082.00 & 1885.62 & 71.62 & 24.74 \\
\hline 155 & IL302 & 100 & 2500 & 1500 & 4000 & 10000 & 6412.03 & 2828.43 & 70.91 & 24.71 \\
\hline 156 & IL302 & 100 & 2500 & 1500 & 5000 & 8000 & 5410.16 & 1414.21 & 71.35 & 24.90 \\
\hline 157 & IL302 & 100 & 2500 & 1500 & 5000 & 10000 & 6742.45 & 2357.02 & 70.94 & 24.74 \\
\hline 158 & IL302 & 100 & 2500 & 1500 & 6000 & 8000 & 5744.29 & 942.81 & 69.95 & 25.38 \\
\hline 159 & IL302 & 100 & 2500 & 1500 & 6000 & 10000 & 7085.92 & 1885.62 & 70.35 & 24.81 \\
\hline
\end{tabular}




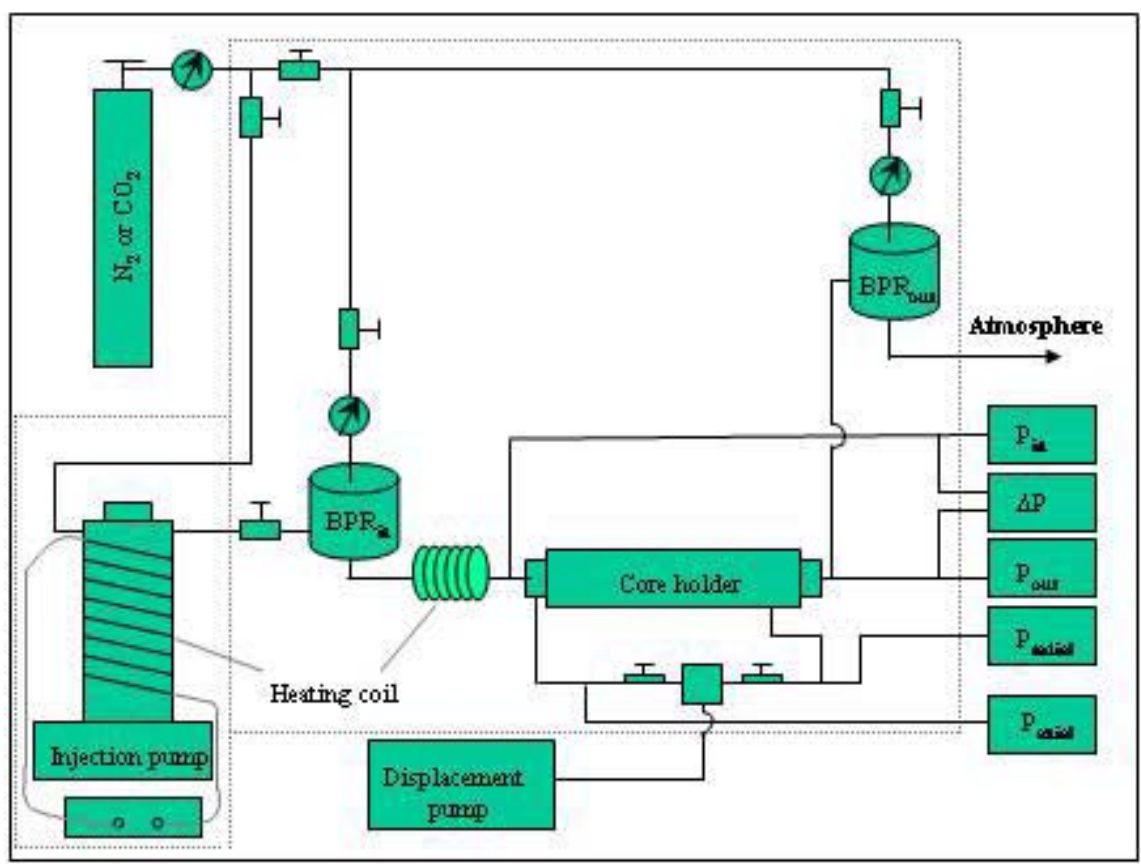

Fig. 5-1. Setup of the experiment.

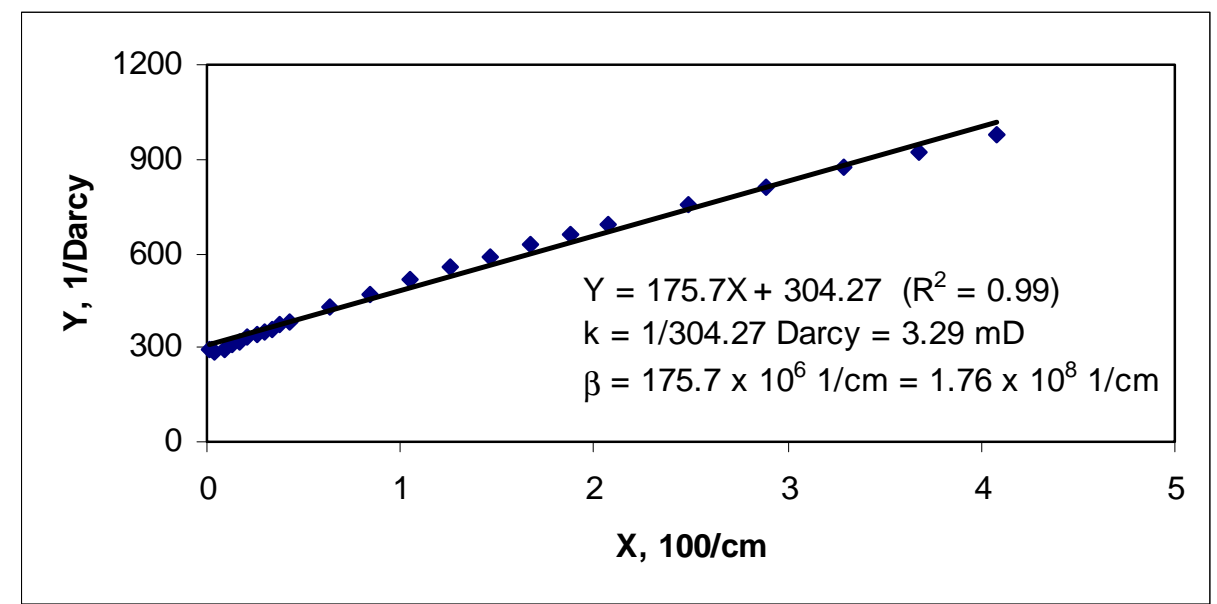

Fig. 5-2. Example of determining $\mathrm{k}$ and $\beta$ from a non-Darcy flow experiment. 


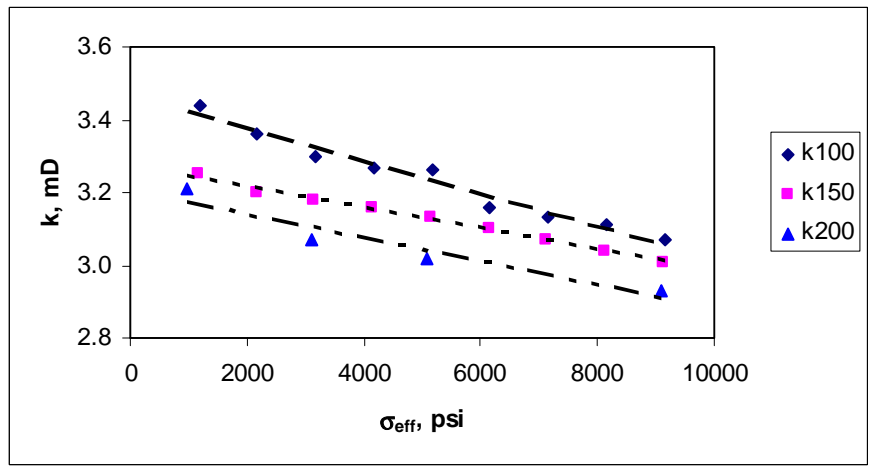

3(a) DSS201

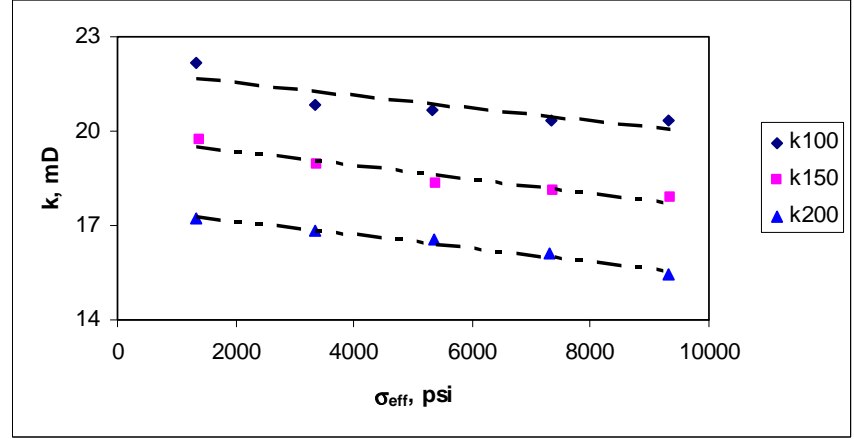

3(b) IL301

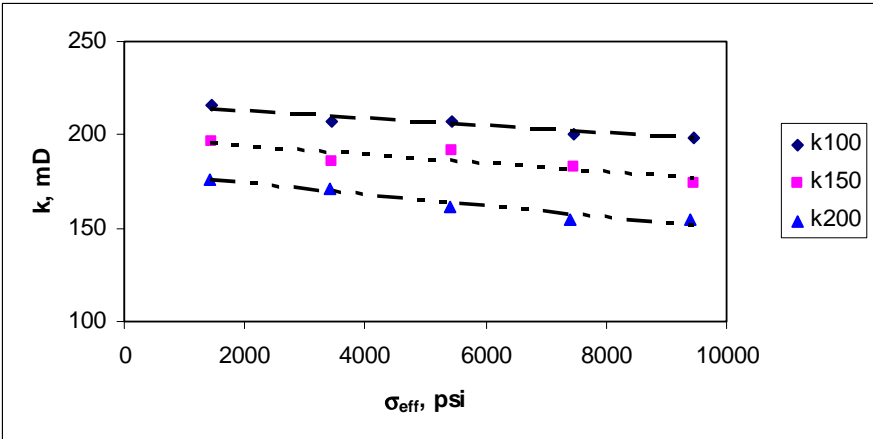

3(c) BSSL301

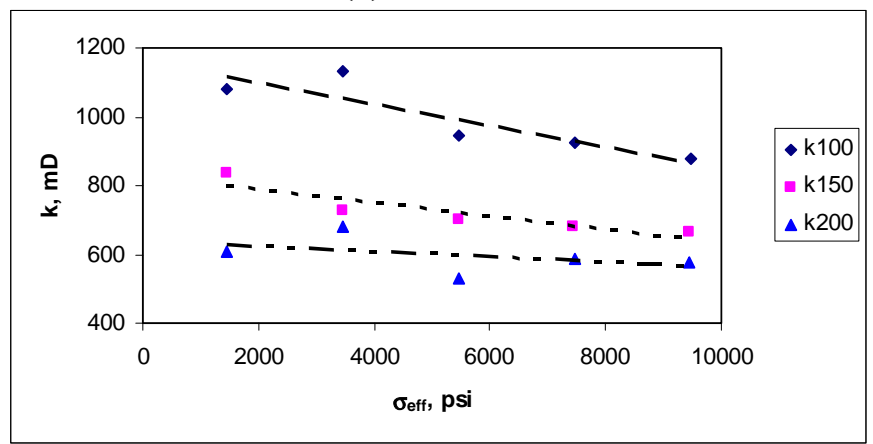

\section{3(d) BSSH301}

Fig. 5-3. Effective stress vs. permeability relations under hydrostatic in-situ stress field at $100^{\circ} \mathrm{F}$, $150^{\circ} \mathrm{F}$ and $200^{\circ} \mathrm{F}$ in four samples. 


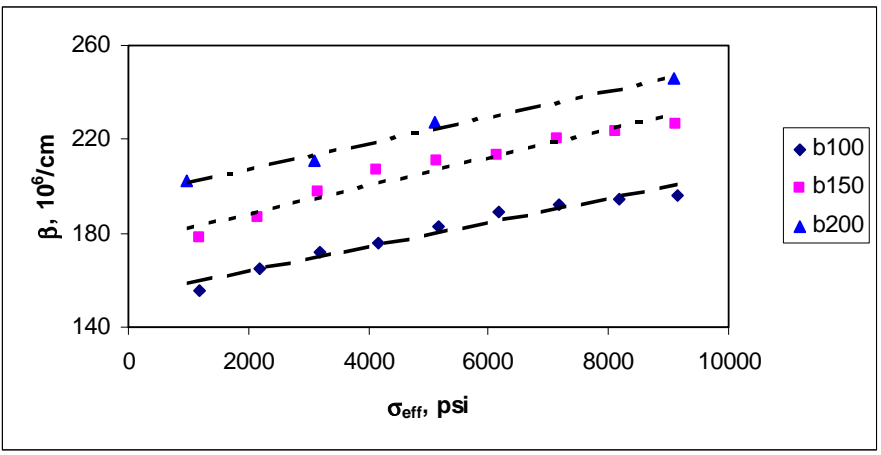

4(a) DSS201

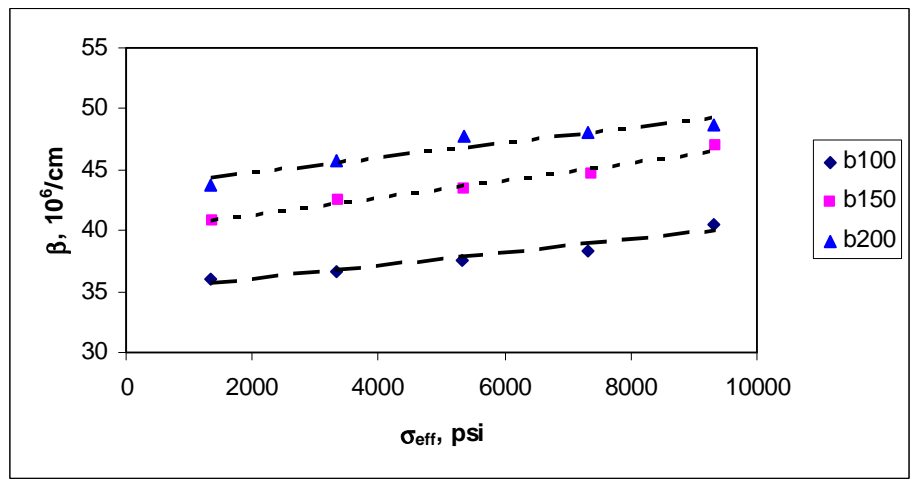

4(b) IL301

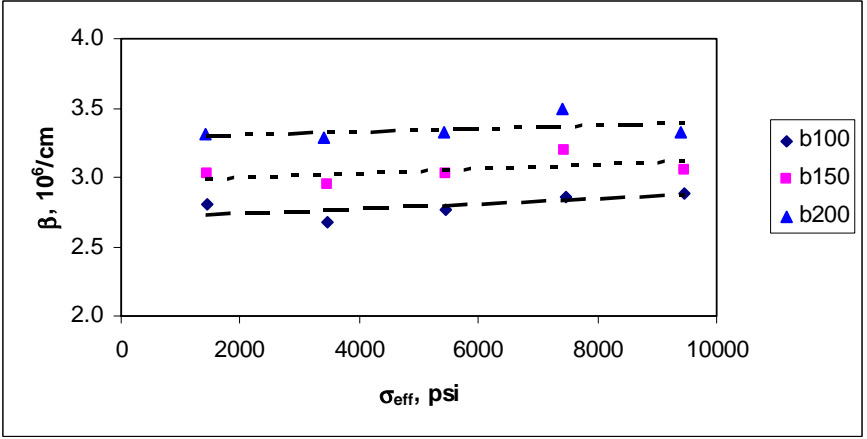

4(c) BSSL301

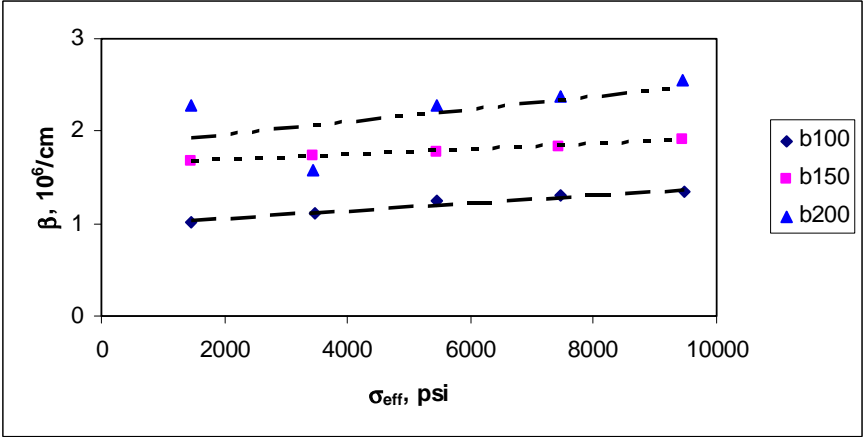

4(d) BSSH301

Fig. 5-4. Effective stress vs. non-Darcy flow coefficient relations under hydrostatic in-situ stress field at $100^{\circ} \mathrm{F}, 150^{\circ} \mathrm{F}$ and $200^{\circ} \mathrm{F}$ in four samples. 


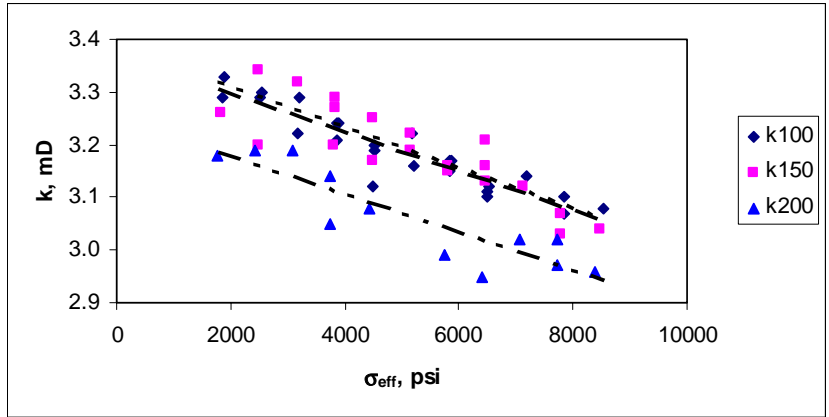

5(a) $\sigma_{\text {eff }}$ vs. $k$

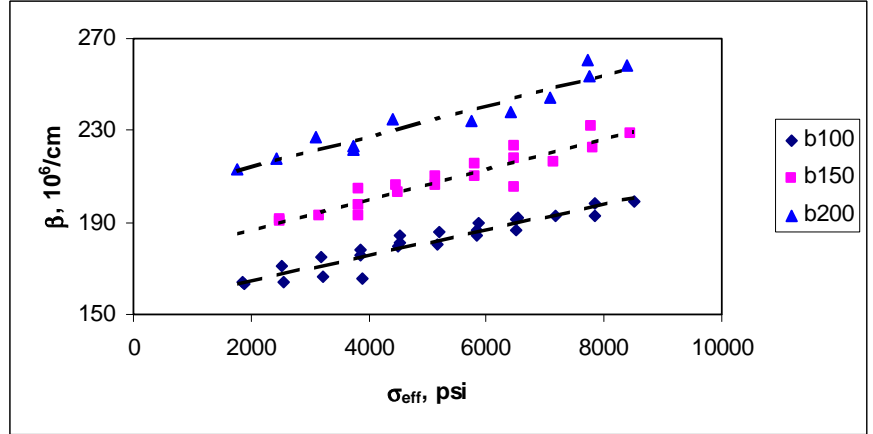

5(b) $\sigma_{\text {eff }}$ Vs. $\beta$

Fig. 5-5. $\mathrm{k}\left(\sigma_{\text {eff }}\right)$ and $\beta\left(\sigma_{\text {eff }}\right)$ relations under differential in-situ stress fields at three temperatures in Dakota sandstone.

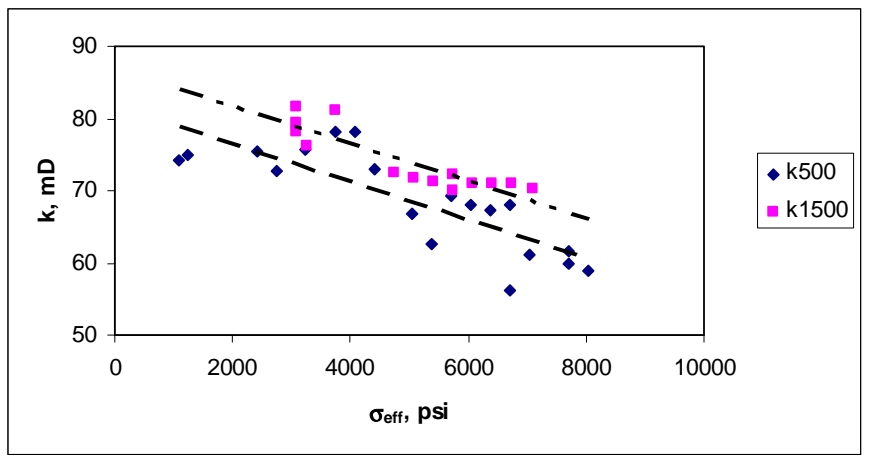

6(a) $\sigma_{\text {eff }}$ vs. $k$

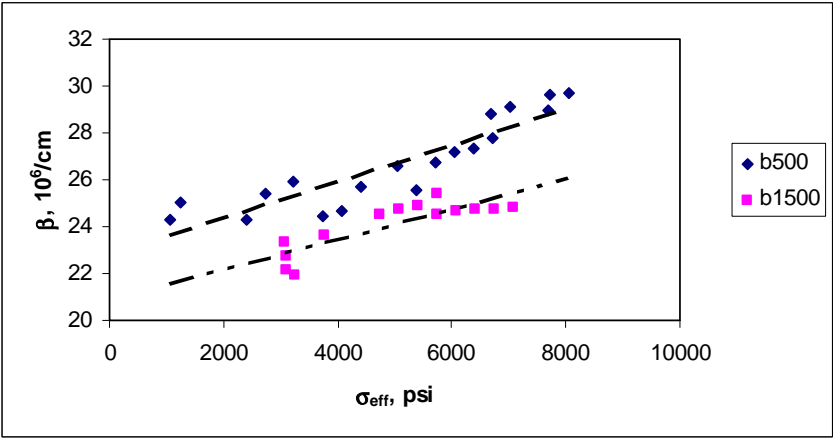

6(b) $\sigma_{\text {eff }}$ Vs. $\beta$

Fig. 5-6. $\sigma_{\text {eff }}-\mathrm{k}$ and $\sigma_{\text {eff }}-\beta$ relations under different reservoir pressures at $100^{\circ} \mathrm{F}$ in high-perm Indian limestone. 


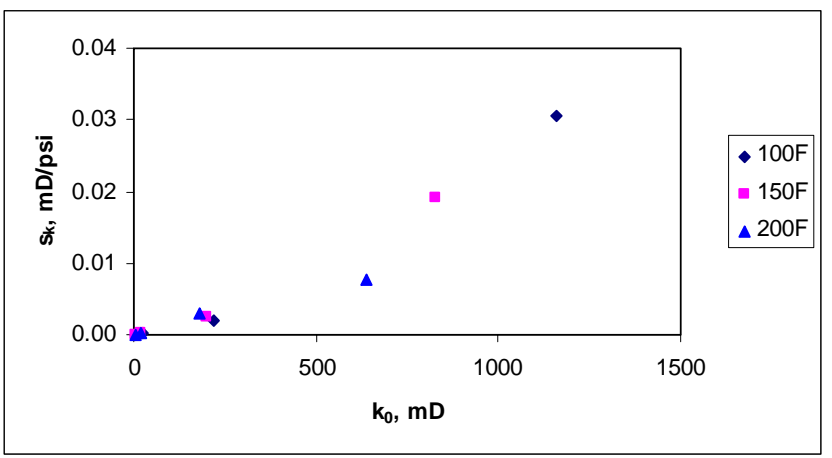

7(a) $k_{0}$ vs. $s_{k}$

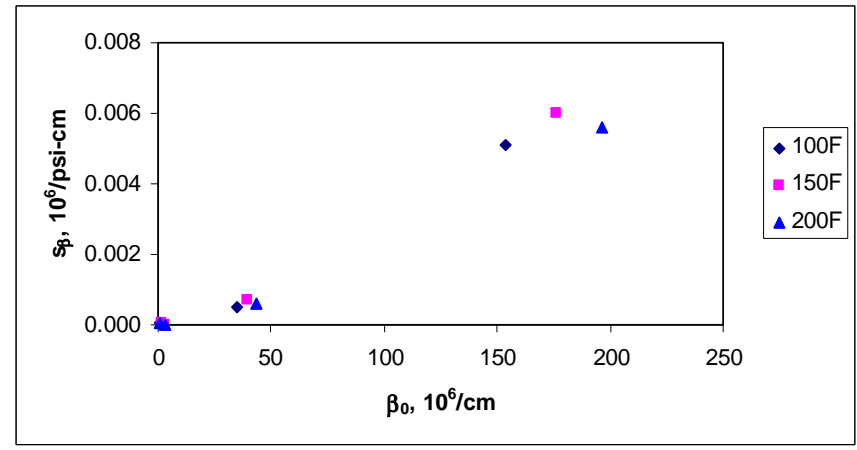

7(b) $\beta_{0}$ vs. $s_{\beta}$

Fig. 5-7. Relations between stress sensitivities and their nominal non-Darcy flow parameters.

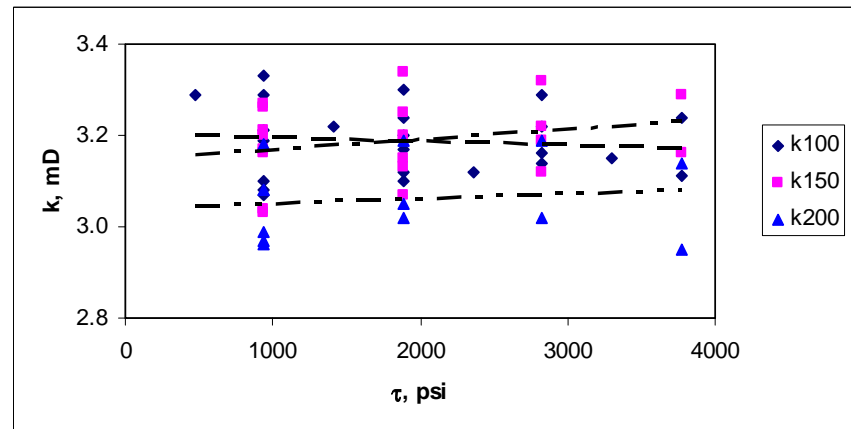

(a) $\tau$ vs. $\mathrm{k}$

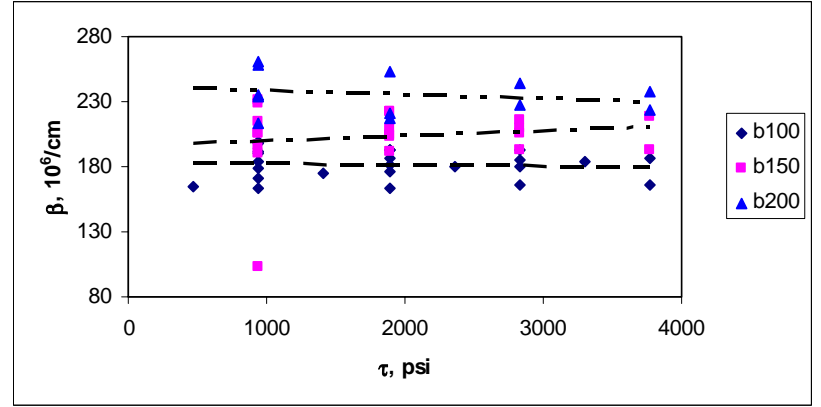

(b) $\tau$ vs. $\beta$

Fig. 5-8. $\tau-\mathrm{k}$ and $\tau-\beta$ curves under temperatures of $100^{\circ} \mathrm{F}, 150^{\circ} \mathrm{F}$ and $200^{\circ} \mathrm{F}$ in Dakota sandstone: widely scattered distribution of the points indicates poor correlation. 


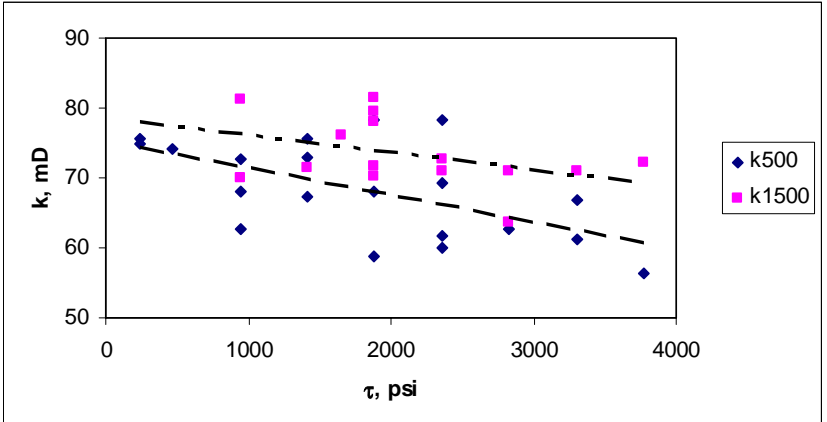

9(a) $\tau$ vs. $k$

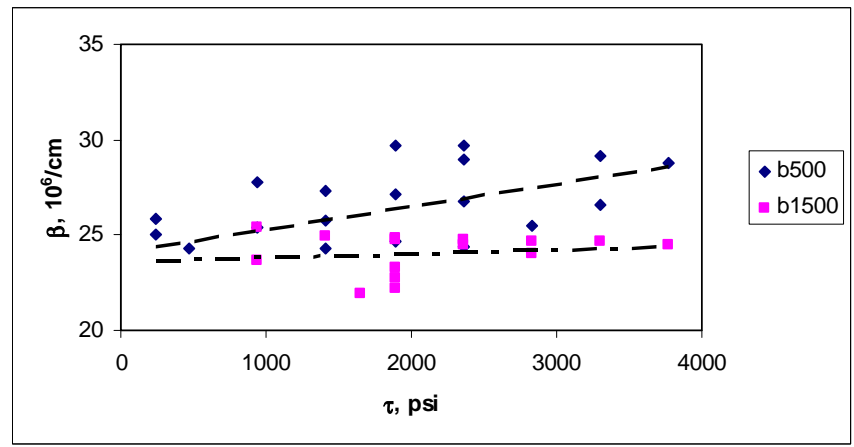

9(b) $\tau$ vs. $\beta$

Fig. 5-9. $\tau-\mathrm{k}$ and $\tau-\beta$ curves under outlet BPR pressures of $500 \mathrm{psi}$ and $1500 \mathrm{psi}$ in high-perm Indiana limestone: widely scattered distribution of the points indicates poor correlation. 


\title{
CHAPTER 6. INJECTIVITY LOSS: COMPARISON OF NON-DARCY FLOW OF $\mathrm{CO}_{2}$ AND N $\mathrm{N}_{2}$ IN A CARBONATE ROCK
}

\author{
Abstract \\ Chapter 6 presents the non-Darcy behavior results of carbon dioxide $\left(\mathrm{CO}_{2}\right)$ compared to the \\ previous work using nitrogen $\left(\mathrm{N}_{2}\right)$ and is based on 85 series of high-velocity gas flooding \\ experiments under high-pressure and high-temperature. It was found that pore pressure has more \\ influence on permeability in $\mathrm{CO}_{2}$ flooding than it does $\mathrm{k}(\tau)$ and $\beta(\tau)$ under differential \\ temperatures in $\mathrm{N}_{2}$ flooding. In contrast, temperature has definite and consistent influence on \\ both permeability and non-Darcy flow coefficient in $\mathrm{N}_{2}$ flooding, but the same influence in $\mathrm{CO}_{2}$ \\ flooding was not observed. The mechanism behind these differences is attributed to physical \\ property differences of the two gases. Much of the work was near the $\mathrm{CO}_{2}$ critical point or liquid \\ regions. Other anomalies are attributed to thermal effects caused by expansion cooling of the \\ $\mathrm{CO}_{2}$. Field data indicates that this phenomenon could be responsible for productivity loses in \\ high flow rate $\mathrm{CO}_{2}$ wells. Accordingly, attention should be paid to avoid flowing $\mathrm{CO}_{2}$ at \\ conditions near its critical point.
}

\section{Introduction}

The study of carbon dioxide $\left(\mathrm{CO}_{2}\right)$ flow behavior in porous media is stimulated by two motivations: to increase oil production from matured reservoirs by using improved oil recovery via $\mathrm{CO}_{2}$ flooding ${ }^{1}$ and to reduce $\mathrm{CO}_{2}$ emissions to the atmosphere by storing it in geological formations via $\mathrm{CO}_{2}$ sequestration. ${ }^{2}$

An accurate characterization of the flow behavior of $\mathrm{CO}_{2}$ in the targeted rock formations is essential to the success of both $\mathrm{CO}_{2}$ flooding and/or $\mathrm{CO}_{2}$ sequestration. Loss of injectivity and productivity in $\mathrm{CO}_{2}$ flooding has been observed in field operations. ${ }^{3}$ Example field data show superficial velocity of $\mathrm{CO}_{2}$ at the near wellbore region varying from $21 \mathrm{ft} /$ day to $1170 \mathrm{ft} / \mathrm{day}^{4}$ For a typical low permeability Indiana limestone at $100^{\circ} \mathrm{F}$ and 1500 psi, the higher velocity corresponds to a Forchheimer number of 3.11, much higher than the proposed critical value of 0.1 for significant non-Darcy effect. ${ }^{5}$ This suggestion that, in some cases, non-Darcy behavior contributes to reduced injectivity and productivity. 
Measurement of permeability and the non-Darcy flow coefficient is needed for the proper quantification of non-Darcy effects. Typically, these values are measured using nitrogen $\left(\mathrm{N}_{2}\right)$ or other inert gases, similar to the measurement of permeability under Darcy flow conditions. ${ }^{6}$ In many other studies, the properties of the gas were not even mentioned. ${ }^{7}$

The non-Darcy flow coefficient has been determined to be not only a property of the rock, but also a property of the fluid, based on non-Darcy flow experiments using air, $\mathrm{N}_{2}$, oxygen and helium. ${ }^{8}$ This leads to a question: how does the non-Darcy flow behavior of $\mathrm{CO}_{2}$ differ from that of $\mathrm{N}_{2}$ ? This chapter presents results of the experimental investigation on this question, the analysis of the mechanism for the difference, and a discussion on the field significance of these observations.

\section{Experimental}

\section{$\mathrm{N}_{2}$ Flooding}

Non-Darcy flow experiments were conducted using a high-pressure, high-temperature, high-flow velocity gas flooding device. Details of the equipment, experimental procedures and data process method about $\mathrm{N}_{2}$ flooding experiments are given elsewhere., ${ }^{4,910}$ This study focuses on modifications for $\mathrm{CO}_{2}$ flooding.

\section{$\mathrm{CO}_{2}$ Flooding}

Because of the special gas properties (phase behavior) of $\mathrm{CO}_{2}$, modifications were made to the equipment, experimental procedures, and the data processing.

Equipment Modifications. First, $\mathrm{CO}_{2}$ was heated in the storage tank up to $85^{\circ} \mathrm{F}$ before refilling the injection pump. The reason for this modification was to have the injection pump at a lower temperature, thus refilling with a liquid and saving refill time. The $\mathrm{CO}_{2}$ heating was realized by winding a heating belt around the bottom of tank. Temperature was monitored using a Type-T Thermalcouple Thermometer.

Next, a constant refill rate of $200 \mathrm{cc} / \mathrm{hr}$ was used to minimize the cooling effect from gas 
expansion. During refill, the cylinder temperature of the injection pump was monitored. A lower temperature was maintained in the injection pump (receiving vessel) to ensure that liquid $\mathrm{CO}_{2}$ and not gaseous $\mathrm{CO}_{2}$ was being transferred.

A mini-thermal heating/cooling system was then added by winding circulating tubing around the cylinder of the injection pump and connecting to a thermally-controlled lead pump. This miniheating/cooling system improved the temperature stability of the injection pump $\mathrm{CO}_{2}$ cylinder.

Finally, the exhaust $\mathrm{CO}_{2}$ was released to a hot water filled bucket, instead of into the atmosphere. This step was to avoid "plugging-explosion" at the outlet tubing due to cooling-induced freezing.

Modifications for Experimental Procedures. The major modifications for the flooding process were to monitor the temperatures at the inlet and outlet of the coreholder and to restore thermal equilibrium of the system to the pre-set temperature after each flow rate. During the interval of thermal re-equilibrium, $\mathrm{CO}_{2}$ was allowed to flow through the core sample at a low flow rate (100 $\mathrm{cc} / \mathrm{hr})$.

Modifications for Data Processing. $\mathrm{CO}_{2}$ properties (density, viscosity and z-factor) were calculated at the injection pump and inside the core were calculated using a commercial PVT simulator with the Peng-Robinson-78 Equation of State. ${ }^{11}$ Pressure and temperature of $\mathrm{CO}_{2}$ in the core were the average of the respective values at the inlet and outlet of the coreholder.

Non-Darcy $\mathrm{N}_{2}$ flooding and $\mathrm{CO}_{2}$ flooding experiments were conducted on a low-permeability (IL301) and a high-permeability Indiana limestone (IL302). The flow rate at the injection pump ranged from $25 \mathrm{cc} / \mathrm{hr}$ to $10000 \mathrm{cc} / \mathrm{hr}$. Three different temperatures $-100^{\circ} \mathrm{F}, 150^{\circ} \mathrm{F}$ and $200^{\circ} \mathrm{F}$-were applied to the coreholder. Three core outlet pressures-500 psi, $1000 \mathrm{psi}$ and $1500 \mathrm{psi}$-were used with corresponding inlet (accumulator) pressures - $2000 \mathrm{psi}, 2000 \mathrm{psi}$, and $2500 \mathrm{psi}-$ respectively. Overburden pressures varied from 2000 psi to 10000 psi with radial and axial directions each independently adjustable. In total, 85 series of flooding experiment were completed for this study, as shown in Table 6-1. 
Effective stresses, instead of overburden, were used to represent the in-situ stress influence. The effective stress in a specific direction was defined as the total in-situ stress in that direction minus the pore pressure. ${ }^{9}$ The average effective stress was defined as the average of the three principal effective stresses. Average shear stress was used to address the influence of shear deformation and was calculated using the octahedral shear stress formula. ${ }^{9}$

Modified Forchheimer's equation ${ }^{12,13}$

$\frac{M\left(p_{1}{ }^{2}-p_{2}^{2}\right)}{2 z R T \mu l\left(\frac{W}{A}\right)}=\frac{1}{k}+\beta\left(\frac{W}{\mu A}\right)$

was used to calculate permeability, $\mathrm{k}$, and non-Darcy flow coefficient, $\beta$, as detailed elsewhere. ${ }^{9,10}$ By denoting $[\mathrm{W} /(\mu \mathrm{A})]$ as $\mathrm{X}$, and $\left\{\mathrm{M}\left(\mathrm{p}_{1}{ }^{2}-\mathrm{p}_{2}{ }^{2}\right) /[2 \mathrm{zRTml}(\mathrm{W} / \mathrm{A})]\right\}$ as $\mathrm{Y}$, Eq. (1) becomes:

$Y=\frac{1}{k}+\beta X$

Using a series of calculated $(\mathrm{X}, \mathrm{Y})$ at different flow rates, $\mathrm{k}$ and $\beta$ are determined from the intercept and the slope of the regressed trend line. Figure 6-1 shows the distribution of (X, Y) and the trend lines for the calculation of $\mathrm{k}$ and $\beta$ for $\mathrm{N}_{2}$ and $\mathrm{CO}_{2}$ flooding under effective stress of about 3300 psi. The trend line equation for $\mathrm{N}_{2}$ flooding points is:

$\mathrm{Y}_{\mathrm{N} 2}=36.91 \mathrm{X}+49.35 \quad\left(\mathrm{R}^{2}=0.99\right)$

According to Eq. (3), the non-Darcy flow parameters are:

$$
\begin{aligned}
& \mathrm{k}_{\mathrm{N} 2}=1 / 49.35 \text { Darcy }=20.26 \mathrm{mD}, \text { and } \\
& \beta_{\mathrm{N} 2}=36.91 \times 10^{6} \mathrm{~cm}^{-1} .
\end{aligned}
$$

Similarly, the trend line for the $\mathrm{CO}_{2}$ flooding points is

$$
\mathrm{Y}_{\mathrm{CO} 2}=39.66 \mathrm{X}+79.02 \quad\left(\mathrm{R}^{2}=0.95\right)
$$

Permeability and non-Darcy flow coefficient corresponding to Eq. (4) are:

$$
\begin{aligned}
& \mathrm{k}_{\mathrm{CO} 2}=1 / 79.02 \text { Darcy }=12.65 \mathrm{mD}, \text { and } \\
& \beta_{\mathrm{CO} 2}=39.66 \times 10^{6} \mathrm{~cm}^{-1} .
\end{aligned}
$$

All the $\mathrm{N}_{2}$ and $\mathrm{CO}_{2}$ flooding experiments under different pressures, temperatures, and in-situ stress fields were processed, as demonstration in the previous cases. Table 6-1 summarizes the calculated results for each series. 


\section{Comparisons of Tested Results from $\mathrm{N}_{2^{-}}$and $\mathrm{CO}_{2^{-}}$Flooding}

Independence of Shear Stresses. Figure 6-1 shows that under the same effective stress, permeability and non-Darcy flow coefficient calculated from $\mathrm{N}_{2}$ and $\mathrm{CO}_{2}$ flooding appear to be different, with $\mathrm{k}_{\mathrm{CO} 2}$ being lower than $\mathrm{k}_{\mathrm{N} 2}$. As shown in Table 6-1, the $\mathrm{N}_{2}$ flooding experiments were run under hydrostatic in-situ stress fields, while $\mathrm{CO}_{2}$ flooding experiments were conducted under differential in-situ stress fields. Before the difference of $\mathrm{k}_{\mathrm{N} 2}$ and $\mathrm{k}_{\mathrm{CO} 2}$ can be attributed to different gas properties, it is necessary to eliminate the contribution of the differences in the insitu stress fields.

Using the shear stress and the calculated non-Darcy parameters of $\mathrm{CO}_{2}$ flooding at $100^{\circ} \mathrm{F}$ in sample IL301, the shear stress influences on $\mathrm{k}_{\mathrm{CO} 2}$ and $\beta_{\mathrm{CO} 2}$ are shown in Fig. 6-2. The scattered distribution of the $\left(\tau, \mathrm{k}_{\mathrm{CO} 2}\right)$ and $\left(\tau, \beta_{\mathrm{CO} 2}\right)$ points in Fig. 6-2 indicates poor $\tau-\mathrm{k}_{\mathrm{CO} 2}$ and $\tau-\beta_{\mathrm{CO} 2}$ correlations. This is further confirmed by the very small value of coefficients of determination, $\mathrm{R}_{\mathrm{k}}{ }^{2}$ and $\mathrm{R}_{\beta}{ }^{2}$, in the following $\mathrm{k}_{\mathrm{CO} 2}(\tau)$ and $\beta_{\mathrm{CO} 2}(\tau)$ regressions, Eq. (5):

$$
\begin{cases}k_{C O 2}=0.0002 \tau+12.49 & \left(\mathrm{R}_{\mathrm{k}}^{2}=0.13\right) \\ \beta_{\mathrm{CO} 2}=-0.0002 \tau+40.28 & \left(\mathrm{R}_{\beta}^{2}=0.01\right)\end{cases}
$$

The above analysis indicates that $\mathrm{k}$ and $\beta$ are independent of shear stress in $\mathrm{CO}_{2}$ flooding, similar to that in $\mathrm{N}_{2}$ flooding. 9,10

Due to this shear stress independence, the difference between $\mathrm{k}_{\mathrm{N} 2}, \beta_{\mathrm{N} 2}$ and $\mathrm{k}_{\mathrm{CO} 2}, \beta_{\mathrm{CO} 2}$ seem to be due to the differences of the gas properties between $\mathrm{N}_{2}$ and $\mathrm{CO}_{2}$.

Results under the Same Effective Stress. Another question arising from Fig. 6-1 is whether the difference between $\mathrm{k}_{\mathrm{N} 2}$ and $\mathrm{k}_{\mathrm{CO} 2}$ is a general phenomenon or a specific case. To answer this question, another comparison was made between the $\mathrm{N}_{2}$ flooding and $\mathrm{CO}_{2}$ flooding experiments under similar effective stresses: $\sigma_{\mathrm{eff}}=5300$ psi, as shown in Fig. 6-3. The regression equations for the $\mathrm{N}_{2}$ - and $\mathrm{CO}_{2}$-flooding experimental data are:

$$
\begin{aligned}
& \mathrm{Y}_{\mathrm{N} 2}=38.31 \mathrm{X}+48.40 \quad\left(\mathrm{R}^{2}=0.99\right) \\
& \mathrm{Y}_{\mathrm{CO} 2}=41.36 \mathrm{X}+77.52 \quad\left(\mathrm{R}^{2}=0.96\right)
\end{aligned}
$$

From this, $\mathrm{k}$ and $\beta$ are calculated as follows:

$$
\mathrm{k}_{\mathrm{N} 2}=1 / 48.40 \text { Darcy }=20.66 \mathrm{mD}
$$




$$
\beta_{\mathrm{N} 2}=38.31 \times 10^{6} \mathrm{~cm}^{-1}
$$

and

$$
\begin{aligned}
& \mathrm{k}_{\mathrm{CO} 2}=1 / 77.52 \text { Darcy }=12.90 \mathrm{mD} \\
& \beta_{\mathrm{CO} 2}=41.36 \times 10^{6} \mathrm{~cm}^{-1}
\end{aligned}
$$

Using Eq. (1) the observation from Fig. 6-1 is repeated here: there is a significant difference between $\mathrm{k}_{\mathrm{N} 2}$ and $\mathrm{k}_{\mathrm{CO} 2}$, and a small difference between $\beta_{\mathrm{N} 2}$ and $\beta_{\mathrm{CO} 2}$.

From close examination of the data in Figs. 6-1 and 6-3, the $\mathrm{N}_{2}$ data fits a straight line much better than does the $\mathrm{CO}_{2}$ data. Both sets have some curvature, but the $\mathrm{CO}_{2}$ data is much more pronounced. Each data set is a better fit to a Forchheimer type equation with a third term. ${ }^{7,12}$ When modified in a similar manner as Eq. (1) the form is:

$$
\frac{M\left(p_{1}{ }^{2}-p_{2}{ }^{2}\right)}{2 z R T \mu l\left(\frac{W}{A}\right)}=\frac{1}{k}+\beta\left(\frac{W}{\mu A}\right)+\gamma \mu\left(\frac{W}{\mu A}\right)^{2}
$$

When the mass flow rate in Eq. (8) approaches zero a value for $1 / \mathrm{k}$ is found. Permeability determinations using Eq. (8) for $\mathrm{N}_{2}$ and $\mathrm{CO}_{2}$ compare better than using Eq. (1). At $\sigma_{\text {eff }}=5300$ psi, $\mathrm{k}$ is $23.49 \mathrm{mD}$ and $20.73 \mathrm{mD}$, respectively, both with $\mathrm{R}^{2}$ above 0.99 .

Another area of possible data discrepancies is that the values for $\mathrm{z}$ and $\mu$ used in this work were

calculated and not experimental data. ${ }^{11}$ At temperatures and pressures of this study these values are within a few percent for $\mathrm{N}_{2}$, but can be off by as much as $100 \%$ for $\mathrm{CO}_{2} .100^{\circ} \mathrm{F}$ is near enough to the critical point of $\mathrm{CO}_{2}$ to cause significant problems in calculating physical properties of $\mathrm{CO}_{2}$. Future work will include recalculation of these data using measured properties for $\mathrm{N}_{2}$ and $\mathrm{CO}_{2}$ to ensure that differences are not due to incorrect values of physical properties. Other problems related to working near the critical point of $\mathrm{CO}_{2}$ are discussed in following sections.

\section{Results under Different Effective Stresses}

A more general comparison is made between $\mathrm{k}_{\mathrm{N} 2}$ and $\beta_{\mathrm{N} 2}$ with $\mathrm{k}_{\mathrm{CO} 2}$ and $\beta_{\mathrm{CO} 2}$, as shown in Fig. 64. From Fig. 6-4, it is clear that $\mathrm{k}_{\mathrm{CO} 2}$ is consistently smaller than $\mathrm{k}_{\mathrm{N} 2}$ under different effective stresses, with a difference of about $30 \%$ at the nominal permeability that corresponds to zero effective stress. The difference between $\beta_{\mathrm{N} 2}$ and $\beta_{\mathrm{CO} 2}$ is not significant: quantitatively less than 
$5 \%$ at nominal non-Darcy flow coefficient, corresponding to zero effective stress.

Results under Different Pore Pressures. Pore pressure is found to have increased $\mathrm{k}_{\mathrm{N} 2}$ and decreased $\beta_{\mathrm{N} 2}$ in $\mathrm{N}_{2}$ flooding experiments on a high-permeability limestone, (IL302). ${ }^{10}$ These results are used here to compare with the pore pressure effect on $\mathrm{k}_{\mathrm{CO} 2}$ and $\beta_{\mathrm{CO} 2}$ tested in low permeability Indiana limestone, as shown in Figs. 6-5 and 6-6.

Because the tests were conducted on two different rocks, IL301 and IL302, the comparison focuses on the trend and the relative change of the parameters. Comparing Fig. 6-5(a) with Fig. $6-5(\mathrm{~b})$, it is obvious that pore pressure has a more significant influence on $\mathrm{k}_{\mathrm{CO} 2}$ than on $\mathrm{k}_{\mathrm{N} 2}$. On the other hand, the pore pressure effect on $\beta_{\mathrm{CO} 2}$ does not have a significant difference from that on $\beta_{\mathrm{N} 2}$, as shown in Fig. 6-6. A quantitative comparison better demonstrates the pore pressure effect. The correlations between $\mathrm{k}, \beta$ and effective stress in Figs. 6-5 and 6 are as follows:

$$
\begin{aligned}
& \begin{cases}\mathrm{k}_{500 \_\mathrm{N} 2}=-2.62 \times 10^{-3} \sigma_{e f f}+81.70 & \left(R^{2}=0.68\right) \\
\mathrm{k}_{1500 \_\mathrm{N} 2}=-2.58 \times 10^{-3} \sigma_{\text {eff }}+86.39 & \left(R^{2}=0.78\right)\end{cases} \\
& \begin{cases}\mathrm{k}_{500 \_\mathrm{CO} 2}=-3.0 \times 10^{-4} \sigma_{\text {eff }}+14.15 & \left(R^{2}=0.62\right) \\
\mathrm{k}_{1500 \_\mathrm{CO} 2}=-5.0 \times 10^{-4} \sigma_{\text {eff }}+25.51 & \left(R^{2}=0.85\right)\end{cases}
\end{aligned}
$$

and

$$
\begin{gathered}
\begin{cases}\beta_{500 \_\mathrm{N} 2}=7.77 \times 10^{-4} \sigma_{e f f}+22.79 & \left(R^{2}=0.81\right) \\
\beta_{1500 \_\mathrm{N} 2}=6.45 \times 10^{-4} \sigma_{e f f}+20.86 & \left(R^{2}=0.73\right)\end{cases} \\
\begin{cases}\beta_{500 \_\mathrm{CO} 2}=8.0 \times 10^{-4} \sigma_{e f f}+36.45 & \left(R^{2}=0.48\right) \\
\beta_{1500 \_\mathrm{CO} 2}=1.5 \times 10^{-3} \sigma_{e f f}+31.67 & \left(R^{2}=0.43\right)\end{cases}
\end{gathered}
$$

Using the nominal parameters, which correspond to zero effective stress, the relative changes of $\mathrm{k}_{\mathrm{N} 2}$ and $\mathrm{k}_{\mathrm{CO} 2}, \beta_{\mathrm{N} 2}$ and $\beta_{\mathrm{CO} 2}$ under different pore pressures are calculated using the following formula:

$$
\left\{\begin{array}{l}
\Delta k=\frac{k_{1500}-k_{500}}{k_{500}} \\
\Delta \beta=\frac{\beta_{1500}-\beta_{500}}{\beta_{500}}
\end{array}\right.
$$

The results of the relative changes are shown in Table 6-2. Negative values indicate a decrease. It is clear that pore pressure has a large influence on permeability in $\mathrm{CO}_{2}$ flooding. 
Results under Different Temperatures. A consistent decrease in $\mathrm{k}$ and increase in $\beta$, with temperature has been observed in $\mathrm{N}_{2}$ flooding tests. ${ }^{10}$ These trends are less significant in $\mathrm{CO}_{2}$ flooding experiments, as compared in Figs. 6-7 and 8.

\section{Brief Summary of the Comparisons}

From the above comparison, it is seen that:

(1) Gas properties have a significant influence on permeability, and a minor influence on the non-Darcy flow coefficient. Permeability measured under $\mathrm{N}_{2}$ flooding appears higher than that under $\mathrm{CO}_{2}$ flooding.

(2) Pore pressures have a more significant influence on permeability in $\mathrm{CO}_{2}$ flooding than they have in $\mathrm{N}_{2}$ flooding.

(3) Temperature effects on permeability and the non-Darcy flow coefficient are more significant and better defined in $\mathrm{N}_{2}$ flooding than in $\mathrm{CO}_{2}$ flooding.

(4) Non-Darcy flow parameters are independent of shear stresses in both $\mathrm{N}_{2}$ flooding and $\mathrm{CO}_{2}$ flooding.

\section{Results and Discussion}

\section{Localized Cooling Effect}

There are many differences between the properties of $\mathrm{N}_{2}$ and $\mathrm{CO}_{2}$. From experimental observation, the most obvious difference is their thermal equilibrium stability. In the $\mathrm{N}_{2}$ flooding experiments, the system had a stable thermal equilibrium: once the temperature reached the preset value, even at the highest flow rate $(10000 \mathrm{cc} / \mathrm{hr})$ in the experiment series, the temperature remained constant, as demonstrated in Fig. 6-9. In contrast, the $\mathrm{CO}_{2}$ flooding experiments had very unstable thermal equilibrium due to the Joule-Thomson cooling effect during fluid expansion caused by the high density gradients in the regions tested. Temperatures in the system cooled as the flow rate increased. When the cooling effect was small, the system temperature remained constant.

Depending on the pore pressure and temperature of the system, the cooling effect started at 
different flow rate levels. In the case of $100^{\circ} \mathrm{F}$ temperature and 500 psi pore pressure, the cooling effect started at about $1000 \mathrm{cc} / \mathrm{hr}$, and could not be controllable at and above $5000 \mathrm{cc} / \mathrm{hr}$, as shown in Fig. 6-10.

On further examination of the results shown in Fig. 6-10 and other experimental results, it was found that when the pressure anywhere within the core was close to $1000 \mathrm{psi}$, the temperature decreased drastically and the thermal equilibrium was lost. This was confirmed in a $\mathrm{CO}_{2}$ flooding experiment with outlet pressure at 1000 psi, as shown in Fig. 6-11.

Recalling that the critical point of $\mathrm{CO}_{2}$ is about $1081 \mathrm{psi}$ and $88^{\circ} \mathrm{F},{ }^{14}$ it is obvious that when the pressure at either the inlet or outlet is close to critical conditions or the saturation curve the Joule-Thomson cooling can be a significant factor. At some point, the thermal equilibrium of the system could not be maintained.

For a similar reason, the cooling effect never occurred in the $\mathrm{N}_{2}$ flooding experiments because the critical point of $\mathrm{N}_{2}$ is 493 psi and $-233^{\circ} \mathrm{F},{ }^{14}$ well out of the range of pressures and temperatures in the testing system.

\section{Multiphase Flow}

Figure 6-10 shows that the temperature at the outlet decreased from $100^{\circ} \mathrm{F}$ to $87^{\circ} \mathrm{F}$ while the pressure at the inlet increased from 940 psi to 1044 psi. This is close to the critical point conditions of $\mathrm{CO}_{2}$. Considering the unstable status of the thermal equilibrium in the system, it is very likely that somewhere between the inlet and outlet, liquid $\mathrm{CO}_{2}$ formed with the possibility of multiphase flow occurring. A direct result would be reduction of the relative permeability.

\section{Significance to Field Operations}

The above observation and interpretation of $\mathrm{CO}_{2}$ flow behavior has significance for field operations in $\mathrm{CO}_{2}$ flooding and sequestration. Because the cooling effect starts at very low flow rates when the pore pressure is close to the critical pressure of $\mathrm{CO}_{2}$, attention should be paid to avoid flowing $\mathrm{CO}_{2}$ in this pressure/temperature region. Once the cooling effect starts, it will build on itself by increasing the pressure gradient and developing multiphase flow, and could 
eventually contribute to killing the well. This could be a mechanism that contributes to injectivity and productivity loses in many $\mathrm{CO}_{2}$ flooding wells. Figure 6-12 shows such an example. Plotted are bottomhole pressure and temperature results from production during the puff cycle of a Huff'n'Puff $\mathrm{CO}_{2}$ project. As the bottomhole conditions approached the $\mathrm{CO}_{2}$ critical point conditions, the pressure and temperature accelerated downward. The production stream composition was about $95 \% \mathrm{CO}_{2}$ with a few percent light hydrocarbons and a relatively small volume of free water. The cooling effect enhanced the reduction of the bottomhole pressure. As the bottomhole temperature returned to reservoir conditions, fluid production and bottomhole pressure did not increase as might have been expected. There appeared to be permanent damage.

\section{Conclusions}

Based on 85 series of laboratory experiments, comparisons between the non-Darcy flow behaviors of $\mathrm{N}_{2}$ and $\mathrm{CO}_{2}$ flooding under high-pressure/high temperature revealed that non-Darcy flow parameters in $\mathrm{CO}_{2}$ flooding are different from that in $\mathrm{N}_{2}$ flooding. Mechanisms for the differences have been analyzed, and significance to field operations discussed. In summary, the following conclusions were obtained:

1. Non-Darcy flow parameters measured in $\mathrm{CO}_{2}$ flooding are different from those in $\mathrm{N}_{2}$ flooding while the non-Darcy flow coefficient in $\mathrm{CO}_{2}$ flooding is slightly higher than that in $\mathrm{N}_{2}$ flooding.

2. Pore pressure has an obvious influence on permeability in $\mathrm{CO}_{2}$ flooding; a similar influence on permeability in $\mathrm{N}_{2}$ flooding is less obvious.

3. Temperature shows clear and consistent influence on non-Darcy flow parameters in $\mathrm{N}_{2}$ flooding, but its influence in $\mathrm{CO}_{2}$ flooding is less pronounced.

4. Some difference in non-Darcy flow behavior between $\mathrm{CO}_{2}$ and $\mathrm{N}_{2}$ is attributed to a cooling effect in $\mathrm{CO}_{2}$ flooding. As temperature and pressure within the core approaches critical point conditions of $\mathrm{CO}_{2}$, the cooling effect enhances. Multiphase flow may occur within the core.

5. This cooling effect and the resulting multiphase flow could be responsible for the loss of injectivity and productivity in some $\mathrm{CO}_{2}$ wells. Care should be paid to avoid flowing $\mathrm{CO}_{2}$ at conditions close to the critical point. 


\section{Nomenclature}

\section{English Symbols}

A $=$ cross-sectional area of the sample, $\mathrm{cm}^{2}$

$\mathrm{b} \quad=\beta$ in figure legends

$\mathrm{k} \quad=$ permeability, Darcy $(\mathrm{mD}$ in correlations)

$1=$ sample length, $\mathrm{cm}$

$\mathrm{M}=$ molecular weight, $\mathrm{g} /$ mole

$\mathrm{p}=$ pressure, atm or $\mathrm{psi}$

$\mathrm{P}_{\mathrm{BH}} \quad=$ pressure, bottomhole, psig

$\mathrm{Q}=$ volume flow rate, $\mathrm{cc} / \mathrm{hr}$

$\mathrm{R}=$ universal gas constant, $82.06 \mathrm{~atm}-\mathrm{cm}^{3} / \mathrm{g}-\mathrm{mole}-\mathrm{K}$

$\mathrm{R}^{2} \quad=$ coefficient of determination

$\mathrm{T} \quad=$ temperature, ${ }^{\circ} \mathrm{K}$ or ${ }^{\circ} \mathrm{F}$

$\mathrm{T}_{\mathrm{BH}}=$ temperature, Bottomhole, ${ }^{\circ} \mathrm{F}$

$\mathrm{W}=$ mass flow rate, $\mathrm{g} / \mathrm{s}$

$\mathrm{X}=$ dummy variable

$\mathrm{Y} \quad=$ dummy variable

$\mathrm{Z}=$ gas compressibility factor

\section{Greek Symbols}

$\beta=$ non-Darcy flow coefficient, atm- $\mathrm{s}^{2} / \mathrm{g}\left(=10^{6} 1 / \mathrm{cm}\right)$

$\gamma \quad=$ Forchheimer equation constant

$\Delta \quad=$ relative change

$\mu \quad=$ fluid viscosity, $\mathrm{cp}$

$\sigma \quad=$ average normal stress, psi

$\tau=$ average shear stress, psi

\section{Subscripts}

$1=$ inlet

$2=$ outlet

$100=$ temperature of $100^{\circ} \mathrm{F}$

$150=$ temperature of $150^{\circ} \mathrm{F}$

$200=$ temperature of $200^{\circ} \mathrm{F}$ 


$$
\begin{array}{ll}
500 & =\text { pore pressure of } 500 \mathrm{psi} \\
1500 & =\text { pore pressure of } 1500 \mathrm{psi} \\
\mathrm{a} & =\text { axial } \\
\mathrm{r} & =\text { radial } \\
\text { eff } & =\text { effective } \\
\mathrm{CO}_{2} & =\mathrm{CO}_{2} \\
\mathrm{~N}_{2} & =\mathrm{N}_{2}
\end{array}
$$

\section{References}

1. Jarrell, P.M., Fox, C.E., Stein, M. H. and Webb, S.L.: Practical Aspects of $\mathrm{CO}_{2}$ Flooding, SPE Monograph Volume 22, Society of Petroleum Engineers, Richardson, TX, USA, 2002.

2. U.S. Department of Energy: Carbon Sequestration Research and Development, Springfield, VA, USA, (Dec. 1999).

3. Rogers, J.D. and Grigg, R.B.: "A Literature Analysis of the WAG Injectivity Abnormalities in the $\mathrm{CO}_{2}$ Process," SPERE, (Oct. 2001) 5(5), 375.

4. Grigg, R.B.: "Improving $\mathrm{CO}_{2}$ Efficiency For Recovering Oil in Heterogeneous Reservoirs," Annual Technical Progress Report DOE Contract No. DE-FG26-01BC15364 (Oct. 2003).

5. Zeng, Z. and Grigg, R.B.: "A Criterion for Non-Darcy Flow in Porous Media," manuscript submitted to Transport in Porous Media.

6. Tiab, D. and Donaldson, E.C.: Petrophysics-Theory and Practice of Measuring Reservoir Rock and Fluid Transport Properties, Gulf Publishing Company, Houston, Texas, (1996).

7. Firoozabadi, A. and Katz, D.L.: "An Analysis of High-Velocity Gas Flow through Porous Media," J. Pet. Tech., (Feb. 1979), 211.

8. Tiss, M. and Evans, R. D.: "Measurement and Correlation of Non-Darcy Flow Coefficient in Consolidated Porous Media," J. Pet. Sci. Eng. (1989), 3, 19-33.

9. Zeng, Z., Grigg, R. and Ganda, S.: "Experimental Study of Overburden and Stress on NonDarcy Gas Flow in Dakota Sandstone," Paper SPE 84069 presented at 2003 SPE Annual Technical Conference and Exhibition, Denver, Oct. 5-8.

10. Zeng, Z., Grigg, R. B. and Gupta, D.B.: "Laboratory Investigation of Stress-Sensitivity of Non-Darcy Gas Flow Parameters," Paper SPE 89431 prepared for presentation at the 2004 SPE/DOE Fourteenth Symposium on Improved Oil Recovery, Tulsa, April 17-21. 
11. PVTsim version 13, 2003, Calsep A/S, GI. Lundtoftevej 1C, DK-2800 Lyngby, Denmark.

12. Forchheimer, P.: “Wasserbewegung durch Boden," Zeit. Ver. Deutsch. Ing., 45, 1901, 17811788.

13. Green, L., Jr. and Duwez, P.: "Fluid Flow through Porous Metals," J. Appl. Mech., (March 1951), 39-45.

14. Ahmed, T.: Hydrocarbon Phase Behavior, Gulf Publishing Company, Houston, Texas, (1989).

Table 6-1. Experiments and Calculated Values

\begin{tabular}{|c|c|c|c|c|c|c|c|c|c|c|c|}
\hline Test & Gas & Rock & $T$ & $P_{\text {in }}$ & $\mathbf{P}_{\text {out }}$ & $\sigma_{a}$ & $\sigma_{\mathrm{r}}$ & $\sigma_{\text {eff }}$ & $\mathbf{T}$ & k & $\tau$ \\
\hline & & & $\mathbf{F}$ & psi & psi & psi & psi & psi & Psi & md & $10^{6} / \mathrm{cm}$ \\
\hline 1 & $\mathrm{~N} 2$ & IL301 & 100 & 2000 & 500 & 2000 & 2000 & 1342.47 & 0.00 & 22.14 & 36.00 \\
\hline 2 & $\mathrm{~N} 2$ & IL301 & 100 & 2000 & 500 & 4000 & 4000 & 3337.40 & 0.00 & 20.82 & 36.61 \\
\hline 3 & N2 & IL301 & 100 & 2000 & 500 & 6000 & 6000 & 5333.93 & 0.00 & 20.66 & 37.55 \\
\hline 4 & $\mathrm{~N} 2$ & IL301 & 100 & 2000 & 500 & 8000 & 8000 & 7332.42 & 0.00 & 20.34 & 38.38 \\
\hline 5 & $\mathrm{~N} 2$ & IL301 & 100 & 2000 & 500 & 10000 & 10000 & 9328.20 & 0.00 & 20.35 & 40.50 \\
\hline & & & & & & & & & & & \\
\hline 6 & $\mathrm{~N} 2$ & IL301 & 150 & 2000 & 500 & 2000 & 2000 & 1376.93 & 0.00 & 19.71 & 40.84 \\
\hline 7 & $\mathrm{~N} 2$ & IL301 & 150 & 2000 & 500 & 4000 & 4000 & 3372.43 & 0.00 & 18.93 & 42.45 \\
\hline 8 & $\mathrm{~N} 2$ & IL301 & 150 & 2000 & 500 & 6000 & 6000 & 5369.91 & 0.00 & 18.36 & 43.44 \\
\hline 9 & $\mathrm{~N} 2$ & IL301 & 150 & 2000 & 500 & 8000 & 8000 & 7367.16 & 0.00 & 18.11 & 44.59 \\
\hline 10 & $\mathrm{~N} 2$ & IL301 & 150 & 2000 & 500 & 10000 & 10000 & 9344.64 & 0.00 & 17.91 & 47.04 \\
\hline & & & & & & & & & & & \\
\hline 11 & $\mathrm{~N} 2$ & IL301 & 200 & 2000 & 500 & 2000 & 2000 & 1333.37 & 0.00 & 17.20 & 43.74 \\
\hline 12 & $\mathrm{~N} 2$ & IL301 & 200 & 2000 & 500 & 4000 & 4000 & 3347.38 & 0.00 & 16.81 & 45.80 \\
\hline 13 & $\mathrm{~N} 2$ & IL301 & 200 & 2000 & 500 & 6000 & 6000 & 5345.40 & 0.00 & 16.58 & 47.74 \\
\hline 14 & $\mathrm{~N} 2$ & IL301 & 200 & 2000 & 500 & 8000 & 8000 & 7317.38 & 0.00 & 16.13 & 48.09 \\
\hline 15 & $\mathrm{~N} 2$ & IL301 & 200 & 2000 & 500 & 10000 & 10000 & 9314.62 & 0.00 & 15.42 & 48.71 \\
\hline & & & & & & & & & & & \\
\hline 16 & $\mathrm{~N} 2$ & IL302 & 100 & 2000 & 500 & 1500 & 2000 & 1239.49 & 235.70 & 74.84 & 25.07 \\
\hline 17 & $\mathrm{~N} 2$ & IL302 & 100 & 2000 & 500 & 1000 & 2000 & 1073.57 & 471.40 & 74.23 & 24.26 \\
\hline 18 & $\mathrm{~N} 2$ & IL302 & 100 & 2000 & 500 & 1000 & 4000 & 2406.40 & 1414.21 & 75.52 & 24.31 \\
\hline 19 & $\mathrm{~N} 2$ & IL302 & 100 & 2000 & 500 & 1000 & 6000 & 3740.25 & 2357.02 & 78.22 & 24.41 \\
\hline 20 & $\mathrm{~N} 2$ & IL302 & 100 & 2000 & 500 & 1000 & 8000 & 5039.09 & 3299.83 & 66.91 & 26.57 \\
\hline 21 & $\mathrm{~N} 2$ & IL302 & 100 & 2000 & 500 & 1000 & 10000 & 6205.66 & 4242.64 & 93.91 & 42.14 \\
\hline 22 & $\mathrm{~N} 2$ & IL302 & 100 & 2000 & 500 & 2000 & 4000 & 2737.64 & 942.81 & 72.75 & 25.42 \\
\hline 23 & $\mathrm{~N} 2$ & IL302 & 100 & 2000 & 500 & 2000 & 6000 & 4073.85 & 1885.62 & 78.25 & 24.67 \\
\hline 24 & $\mathrm{~N} 2$ & IL302 & 100 & 2000 & 500 & 2000 & 8000 & 5373.24 & 2828.43 & 62.59 & 25.52 \\
\hline 25 & $\mathrm{~N} 2$ & IL302 & 100 & 2000 & 500 & 2000 & 10000 & 6699.44 & 3771.24 & 56.23 & 28.82 \\
\hline 26 & $\mathrm{~N} 2$ & IL302 & 100 & 2000 & 500 & 3500 & 4000 & 3236.75 & 235.70 & 75.70 & 25.89 \\
\hline 27 & $\mathrm{~N} 2$ & IL302 & 100 & 2000 & 500 & 3000 & 6000 & 4403.56 & 1414.21 & 73.02 & 25.74 \\
\hline 28 & $\mathrm{~N} 2$ & IL302 & 100 & 2000 & 500 & 3000 & 8000 & 5708.25 & 2357.02 & 69.16 & 26.76 \\
\hline 29 & $\mathrm{~N} 2$ & IL302 & 100 & 2000 & 500 & 3000 & 10000 & 7033.33 & 3299.83 & 61.20 & 29.12 \\
\hline 30 & $\mathrm{~N} 2$ & IL302 & 100 & 2000 & 500 & 4000 & 6000 & 4732.64 & 942.81 & 62.60 & 18.48 \\
\hline 31 & $\mathrm{~N} 2$ & IL302 & 100 & 2000 & 500 & 4000 & 8000 & 6040.58 & 1885.62 & 68.01 & 27.16 \\
\hline 32 & $\mathrm{~N} 2$ & IL302 & 100 & 2000 & 500 & 5000 & 10000 & 7701.24 & 2357.02 & 59.96 & 28.93 \\
\hline 33 & $\mathrm{~N} 2$ & IL302 & 100 & 2000 & 500 & 5000 & 8000 & 6374.41 & 1414.21 & 67.34 & 27.30 \\
\hline 34 & $\mathrm{~N} 2$ & IL302 & 100 & 2000 & 500 & 5000 & 10000 & 7711.49 & 2357.02 & 61.64 & 29.65 \\
\hline 35 & $\mathrm{~N} 2$ & IL302 & 100 & 2000 & 500 & 6000 & 8000 & 6706.01 & 942.81 & 68.01 & 27.76 \\
\hline 36 & $\mathrm{~N} 2$ & IL302 & 100 & 2000 & 500 & 6000 & 10000 & 8044.58 & 1885.62 & 58.89 & 29.67 \\
\hline 37 & $\mathrm{~N} 2$ & IL302 & 100 & 2000 & 1500 & 2500 & 6000 & 3253.10 & 1649.92 & 76.12 & 21.93 \\
\hline 38 & $\mathrm{~N} 2$ & IL302 & 100 & 2000 & 1500 & 2000 & 6000 & 3086.68 & 1885.62 & 81.49 & 22.18 \\
\hline 39 & $\mathrm{~N} 2$ & IL302 & 100 & 2000 & 1500 & 2000 & 8000 & 4406.71 & 2828.43 & 63.67 & 24.00 \\
\hline 40 & $\mathrm{~N} 2$ & IL302 & 100 & 2000 & 1500 & 2000 & 10000 & 5742.51 & 3771.24 & 72.29 & 24.52 \\
\hline 41 & $\mathrm{~N} 2$ & IL302 & 100 & 2000 & 1500 & 2000 & 6000 & 3085.03 & 1885.62 & 78.13 & 23.32 \\
\hline 42 & $\mathrm{~N} 2$ & IL302 & 100 & 2000 & 1500 & 2000 & 6000 & 3089.10 & 1885.62 & 79.42 & 22.73 \\
\hline 43 & $\mathrm{~N} 2$ & IL302 & 100 & 2000 & 1500 & 3000 & 8000 & 4743.67 & 2357.02 & 72.58 & 24.51 \\
\hline 44 & $\mathrm{~N} 2$ & IL302 & 100 & 2000 & 1500 & 3000 & 10000 & 6077.75 & 3299.83 & 71.08 & 24.66 \\
\hline
\end{tabular}




\begin{tabular}{|c|c|c|c|c|c|c|c|c|c|c|c|}
\hline Test & Gas & Rock & $T$ & $P_{\text {in }}$ & $\mathbf{P}_{\text {out }}$ & $\sigma_{a}$ & $\sigma_{r}$ & $\sigma_{\text {eff }}$ & $\mathbf{T}$ & $\mathbf{k}$ & $\tau$ \\
\hline & & & $\mathbf{F}$ & psi & psi & psi & psi & psi & Psi & md & $10^{6} / \mathrm{cm}$ \\
\hline 45 & N2 & IL302 & 100 & 2000 & 1500 & 4000 & 6000 & 3755.07 & 942.81 & 81.21 & 23.66 \\
\hline 46 & N2 & IL302 & 100 & 2000 & 1500 & 4000 & 8000 & 5082.00 & 1885.62 & 71.62 & 24.74 \\
\hline 47 & N2 & IL302 & 100 & 2000 & 1500 & 4000 & 10000 & 6412.03 & 2828.43 & 70.91 & 24.71 \\
\hline 48 & N2 & IL302 & 100 & 2000 & 1500 & 5000 & 8000 & 5410.16 & 1414.21 & 71.35 & 24.90 \\
\hline 49 & N2 & IL302 & 100 & 2000 & 1500 & 5000 & 10000 & 6742.45 & 2357.02 & 70.94 & 24.74 \\
\hline 50 & N2 & IL302 & 100 & 2000 & 1500 & 6000 & 8000 & 5744.29 & 942.81 & 69.95 & 25.38 \\
\hline 51 & $\mathrm{~N} 2$ & IL302 & 100 & 2000 & 1500 & 6000 & 10000 & 7085.92 & 1885.62 & 70.35 & 24.81 \\
\hline 52 & $\mathrm{CO} 2$ & IL301 & 100 & 2500 & 1500 & 6000 & 2000 & 1690.26 & 1885.62 & 25.01 & 33.44 \\
\hline 53 & $\mathrm{CO} 2$ & IL301 & 100 & 2000 & 1500 & 6000 & 3000 & 2372.83 & 1414.21 & 24.01 & 39.69 \\
\hline 54 & $\mathrm{CO} 2$ & IL301 & 100 & 2000 & 1500 & 6000 & 4000 & 3015.64 & 942.81 & 23.67 & 39.24 \\
\hline 55 & $\mathrm{CO} 2$ & IL301 & 100 & 2000 & 1500 & 8000 & 2000 & 2370.82 & 2828.43 & 24.24 & 34.11 \\
\hline 56 & $\mathrm{CO} 2$ & IL301 & 100 & 2000 & 1500 & 8000 & 4000 & 3668.63 & 1885.62 & 23.94 & 35.96 \\
\hline 57 & $\mathrm{CO} 2$ & IL301 & 100 & 2000 & 1500 & 8000 & 6000 & 5005.68 & 942.81 & 23.64 & 40.80 \\
\hline 58 & $\mathrm{CO} 2$ & IL301 & 100 & 2000 & 1500 & 10000 & 2000 & 2988.75 & 3771.24 & 24.36 & 33.80 \\
\hline 59 & $\mathrm{CO} 2$ & IL301 & 100 & 2000 & 1500 & 10000 & 4000 & 4324.82 & 2828.43 & 23.02 & 33.19 \\
\hline 60 & $\mathrm{CO} 2$ & IL301 & 100 & 2000 & 1500 & 10000 & 6000 & 5671.59 & 1885.62 & 22.71 & 38.83 \\
\hline 61 & $\mathrm{CO} 2$ & IL301 & 100 & 2000 & 1500 & 10000 & 8000 & 6927.64 & 942.81 & 22.01 & 44.06 \\
\hline 62 & $\mathrm{CO} 2$ & IL301 & 100 & 2000 & 500 & 4000 & 2000 & 2033.51 & 942.81 & 13.61 & 38.28 \\
\hline 63 & $\mathrm{CO} 2$ & IL301 & 100 & 2500 & 500 & 6000 & 2000 & 2698.99 & 1885.62 & 13.33 & 37.91 \\
\hline 64 & $\mathrm{CO} 2$ & IL301 & 100 & 2000 & 500 & 6000 & 3000 & 3363.29 & 1414.21 & 12.65 & 39.66 \\
\hline 65 & $\mathrm{CO} 2$ & IL301 & 100 & 2000 & 500 & 6000 & 4000 & 4025.94 & 942.81 & 12.48 & 41.95 \\
\hline 66 & $\mathrm{CO} 2$ & IL301 & 100 & 2000 & 500 & 8000 & 2000 & 3364.87 & 2828.43 & 13.77 & 36.82 \\
\hline 67 & $\mathrm{CO} 2$ & IL301 & 100 & 2000 & 500 & 8000 & 4000 & 4690.68 & 1885.62 & 12.69 & 39.71 \\
\hline 68 & $\mathrm{CO} 2$ & IL301 & 100 & 2000 & 500 & 8000 & 6000 & 6028.68 & 942.81 & 12.17 & 41.64 \\
\hline 69 & $\mathrm{CO} 2$ & IL301 & 100 & 2000 & 500 & 10000 & 2000 & 4028.23 & 3771.24 & 13.09 & 40.91 \\
\hline 70 & $\mathrm{CO} 2$ & IL301 & 100 & 2000 & 500 & 10000 & 4000 & 5360.57 & 2828.43 & 12.90 & 41.36 \\
\hline 71 & $\mathrm{CO} 2$ & IL301 & 100 & 2000 & 500 & 10000 & 6000 & 6695.83 & 1885.62 & 12.21 & 40.91 \\
\hline 72 & $\mathrm{CO} 2$ & IL301 & 150 & 2000 & 500 & 6000 & 2000 & 2675.36 & 1885.62 & 14.27 & 43.17 \\
\hline 73 & $\mathrm{CO} 2$ & IL301 & 150 & 2000 & 500 & 6000 & 3000 & 3344.93 & 1414.21 & 12.50 & 44.68 \\
\hline 74 & $\mathrm{CO} 2$ & IL301 & 150 & 2000 & 500 & 6000 & 4000 & 4011.56 & 942.81 & 13.78 & 42.77 \\
\hline 75 & $\mathrm{CO} 2$ & IL301 & 150 & 2000 & 500 & 8000 & 2000 & 3377.19 & 2828.43 & 14.61 & 37.06 \\
\hline 76 & $\mathrm{CO} 2$ & IL301 & 150 & 2000 & 500 & 8000 & 4000 & 4702.69 & 1885.62 & 14.43 & 42.72 \\
\hline 77 & $\mathrm{CO} 2$ & IL301 & 150 & 2000 & 500 & 8000 & 6000 & 6000.59 & 942.81 & 12.62 & 46.04 \\
\hline & & & & & & & & & & & \\
\hline 78 & $\mathrm{CO} 2$ & IL301 & 200 & 2000 & 500 & 6000 & 2000 & 2665.67 & 1885.62 & 15.43 & 50.15 \\
\hline 79 & $\mathrm{CO} 2$ & IL301 & 200 & 2000 & 500 & 6000 & 3000 & 3333.50 & 1414.21 & 14.53 & 47.60 \\
\hline 80 & $\mathrm{CO} 2$ & IL301 & 200 & 2000 & 500 & 6000 & 4000 & 3991.74 & 942.81 & 13.73 & 50.31 \\
\hline 81 & $\mathrm{CO} 2$ & IL301 & 200 & 2000 & 500 & 8000 & 2000 & 3321.42 & 2828.43 & 14.31 & 50.74 \\
\hline 82 & $\mathrm{CO} 2$ & IL301 & 200 & 2000 & 500 & 8000 & 4000 & 4654.80 & 1885.62 & 13.56 & 45.27 \\
\hline 83 & $\mathrm{CO} 2$ & IL301 & 200 & 2000 & 500 & 8000 & 6000 & 5996.75 & 942.81 & 12.66 & 48.08 \\
\hline & & & & & & & & \multirow{2}{*}{\multicolumn{4}{|c|}{ Incomplete due to cooling effect. }} \\
\hline 84 & $\mathrm{CO}_{2}$ & IL301 & 100 & 2000 & 1000 & 4000 & 2000 & & & & \\
\hline 85 & $\mathrm{CO}_{2}$ & IL301 & 100 & 2000 & 1000 & 6000 & 3000 & \multicolumn{4}{|c|}{ Incomplete due to cooling effect. } \\
\hline
\end{tabular}

Table 6-2. Relative Change of $k$ and $\beta$ under Different Pore Pressures

\begin{tabular}{|c|c|c|}
\hline Parameter & Gas & Relative change, \% \\
\hline \multirow{2}{*}{$\mathrm{N}$} & $\mathrm{N}_{2}$ & $6.35 \%$ \\
\cline { 2 - 3 } & $\mathrm{CO}_{2}$ & $80.28 \%$ \\
\hline \multirow{3}{*}{$\beta$} & $\mathrm{N}_{2}$ & $-8.47 \%$ \\
\cline { 2 - 3 } & $\mathrm{CO}_{2}$ & $-11.13 \%$ \\
\hline
\end{tabular}




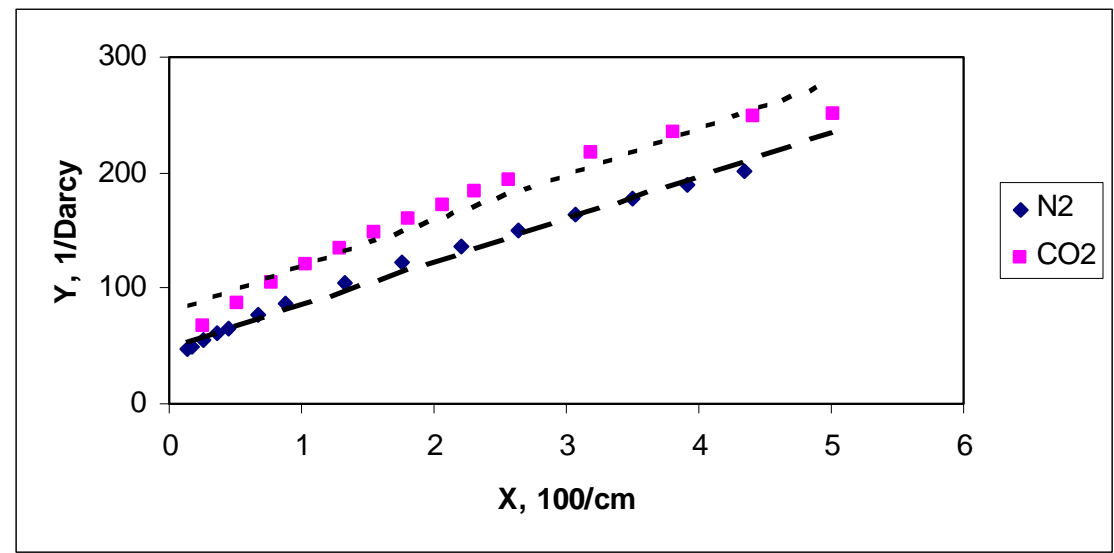

Fig. 6-1. Comparison of trend lines in $\mathrm{N}_{2}$ and $\mathrm{CO}_{2}$ flooding through low permeability Indiana limestone under effective stress of about 3300 psi.

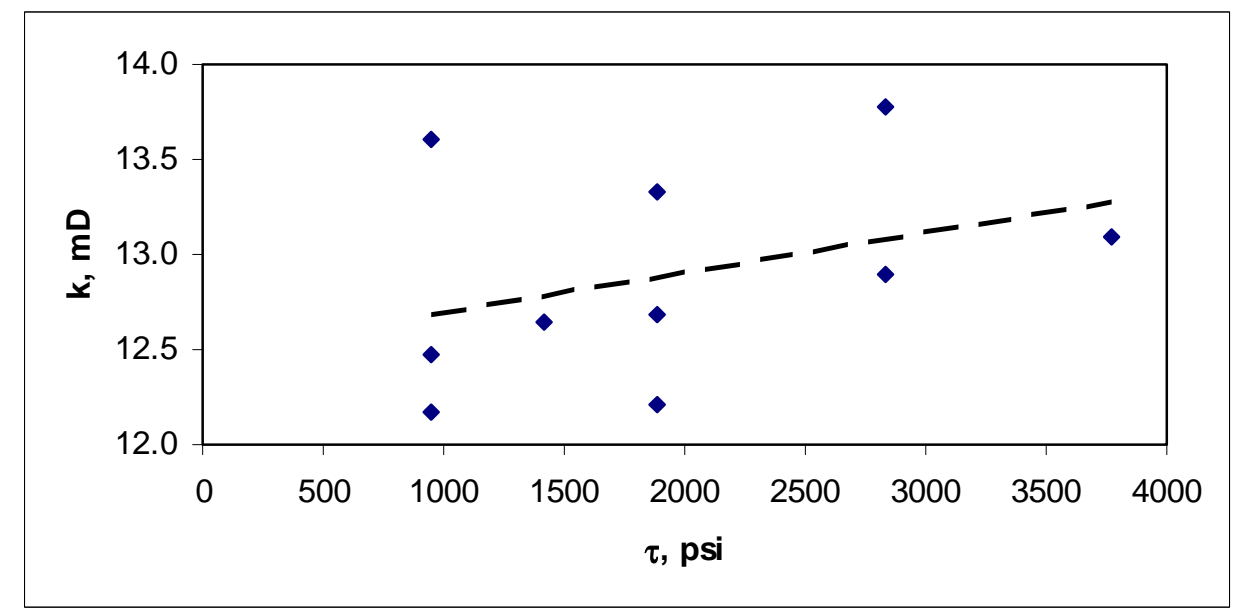

2(a). $\tau$ vs. $k$

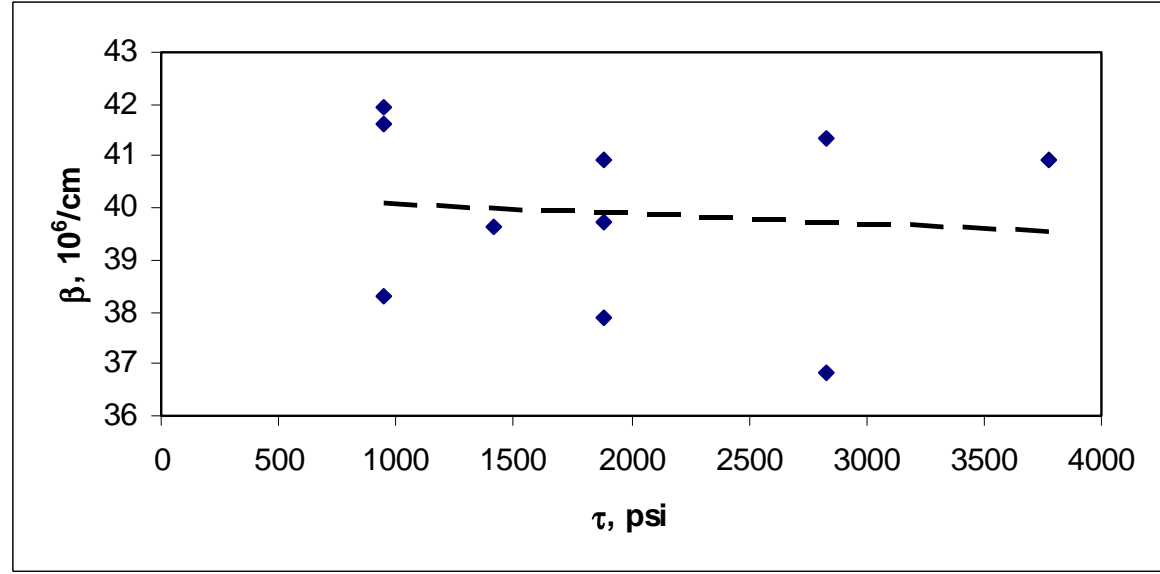

2(b). $\tau$ vs. $\beta$

Fig. 6-2 Relationship between shear stress and non-Darcy flow parameters in IL301 at temperature of $100^{\circ} \mathrm{F}$ : (a) permeability, (b) non-Darcy flow coefficient. 


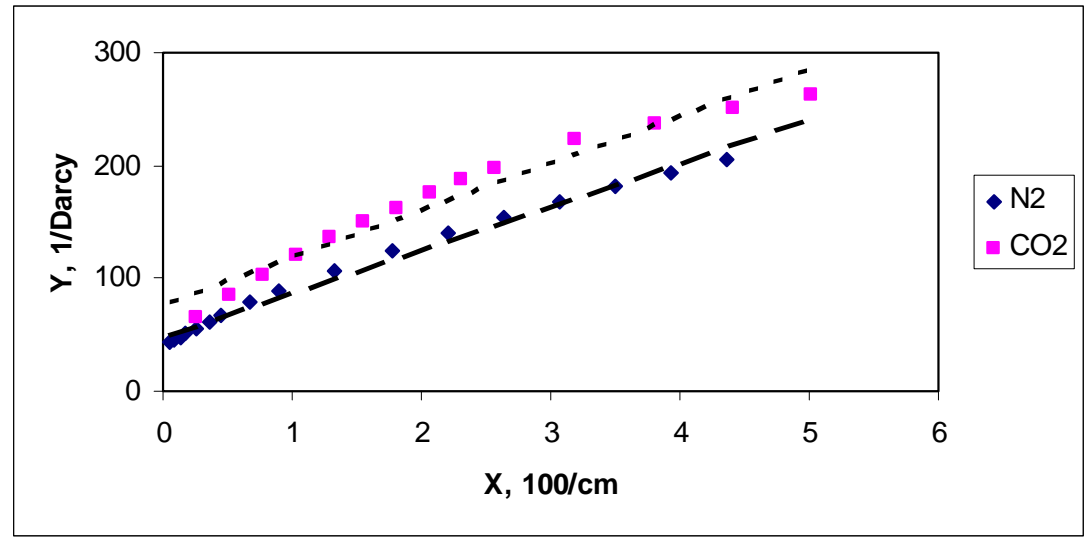

Fig. 6-3. Comparison of trend lines in $\mathrm{N}_{2}$ and $\mathrm{CO}_{2}$ flooding in IL301 under effective stress of about 5300 psi.

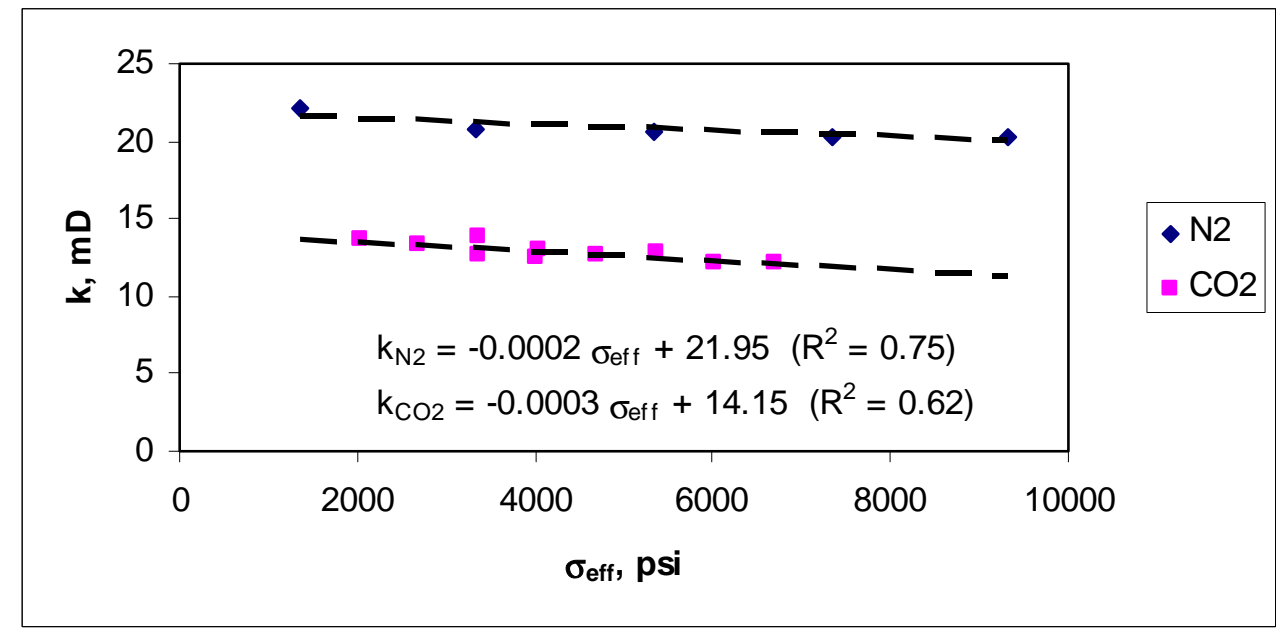

4(a). $\mathrm{k}_{\mathrm{N} 2}$ vs. $\mathrm{k}_{\mathrm{CO} 2}$

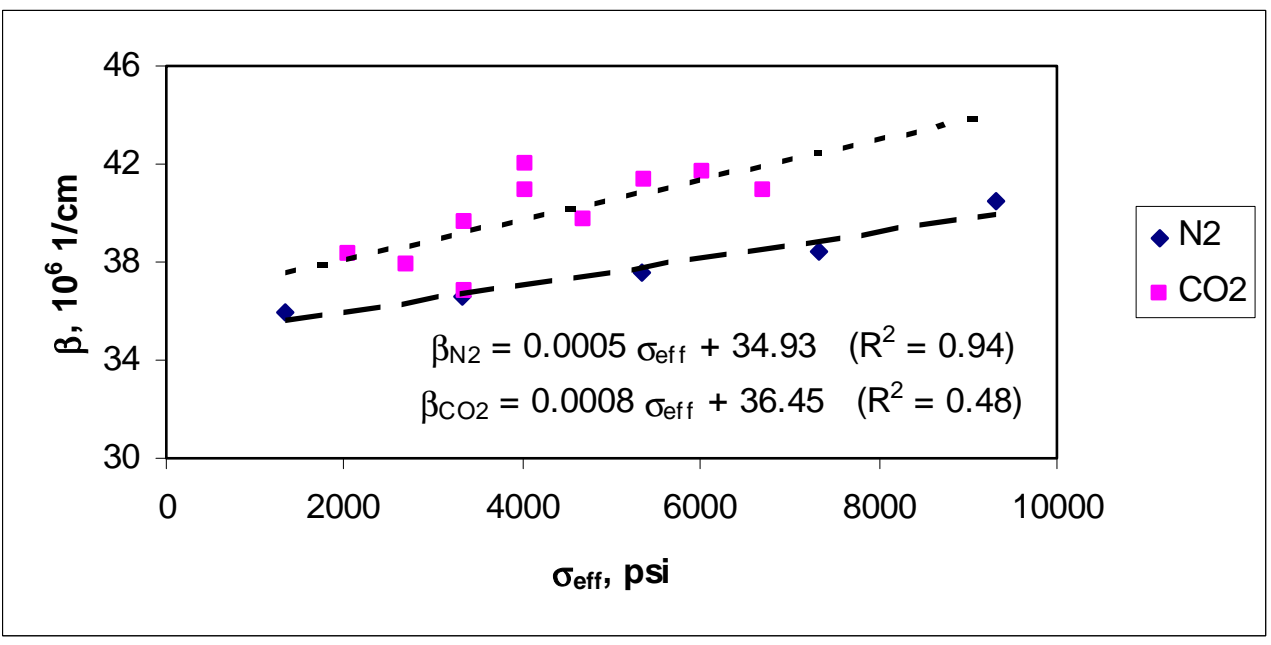

4(b) $\beta_{\mathrm{N} 2}$ vs. $\beta_{\mathrm{CO} 2}$

Fig. 6-4. Comparison of $\left(\mathrm{k}_{\mathrm{N} 2}, \beta_{\mathrm{N} 2}\right)$ and $\left(\mathrm{k}_{\mathrm{CO} 2}, \beta_{\mathrm{CO} 2}\right)$ under different effective stresses. 


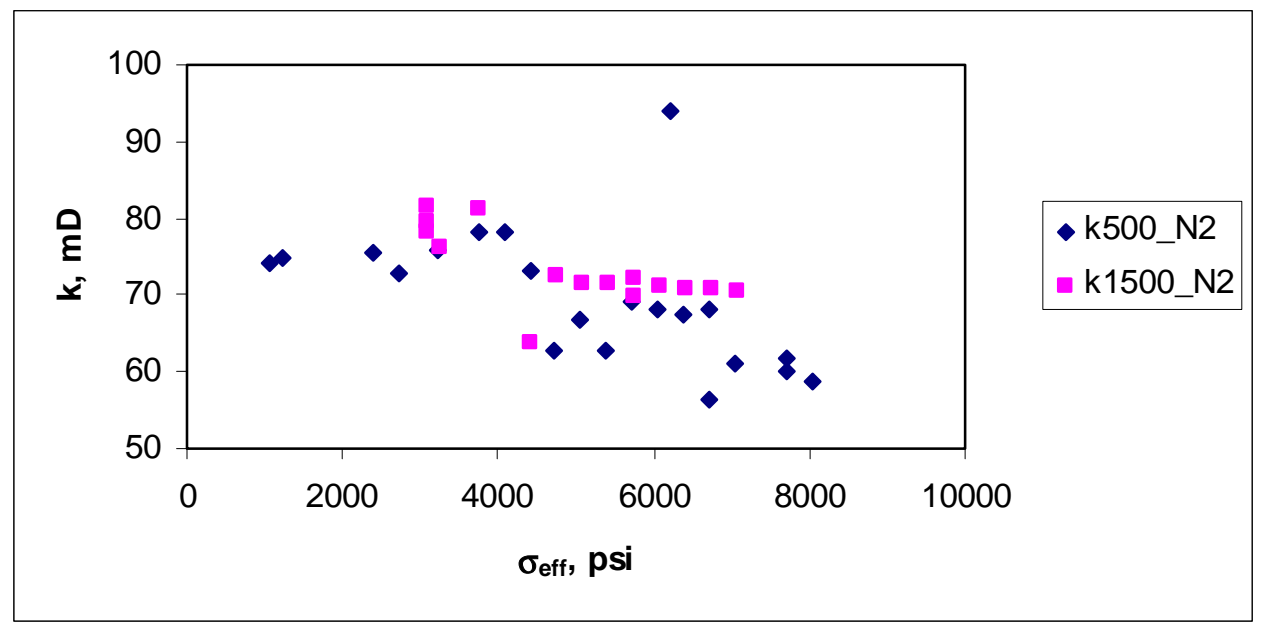

5(a). K500_N2 VS. K1500_N2

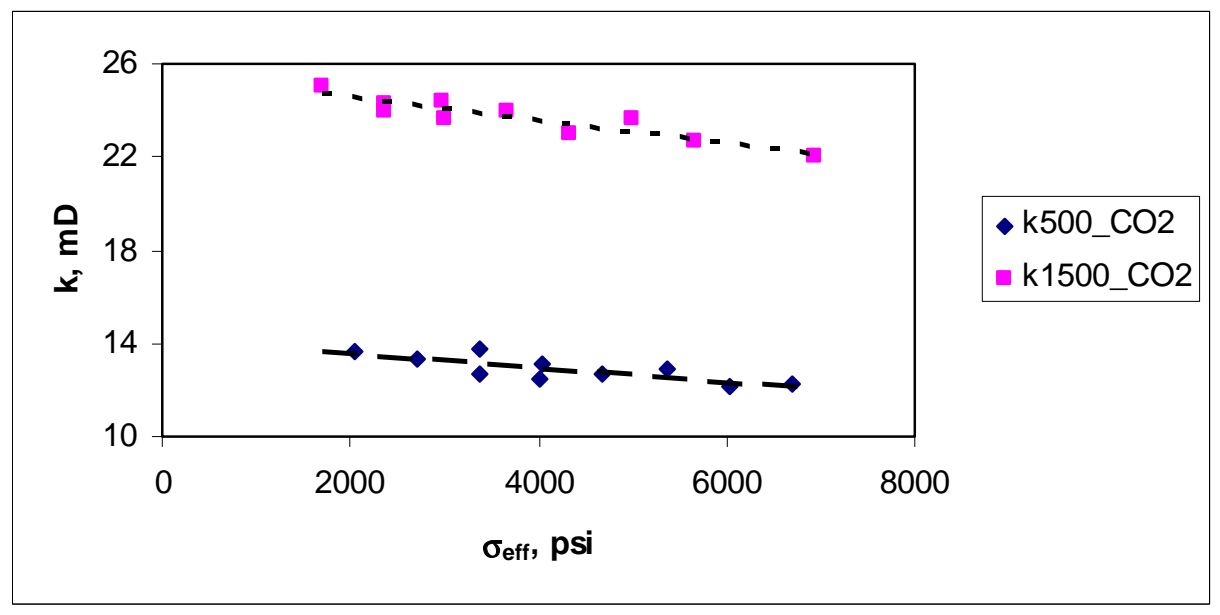

5(b) k500_CO2 VS. K1500_CO2

Fig. 6-5. Comparison of pore pressure effects on permeability in $\mathrm{N}_{2}$ and $\mathrm{CO}_{2}$ flooding. 


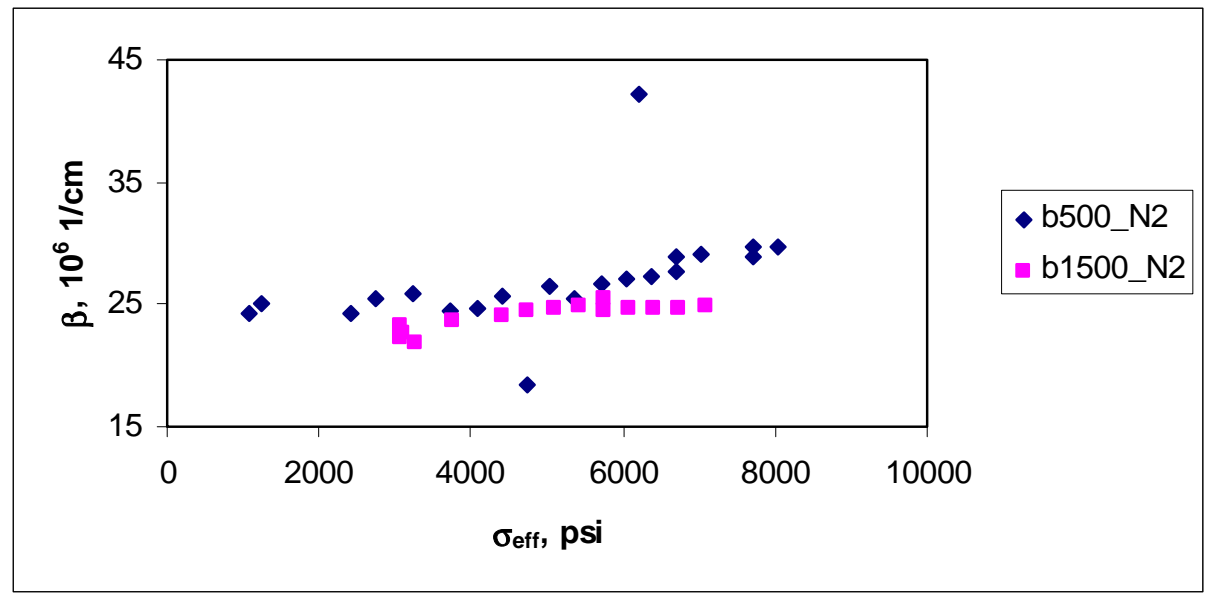

6(a). $\beta_{500 \_N 2}$ VS. $\beta_{1500 \_N 2}$

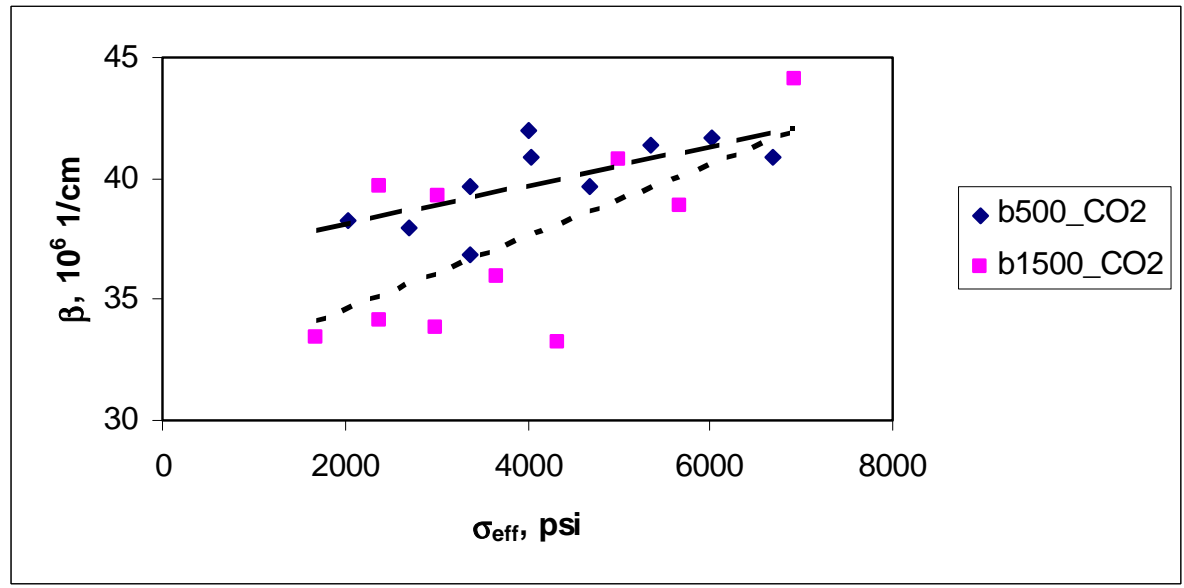

6(b). $\beta_{500 \_C O 2}$ VS. $\beta_{1500 \_C O 2}$

Fig. 6-6. Comparison of pore pressure effects on non-Darcy flow coefficient in $\mathrm{N}_{2}$ and $\mathrm{CO}_{2}$ flooding. 


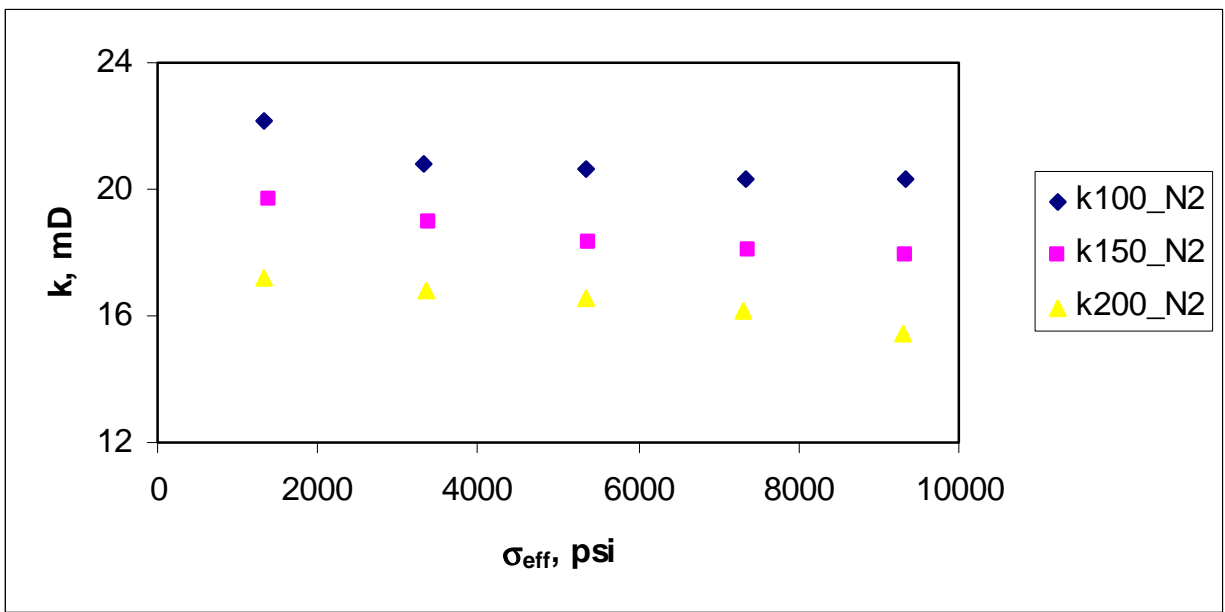

7(a). $k_{\mathrm{N} 2}$ under different temperatures

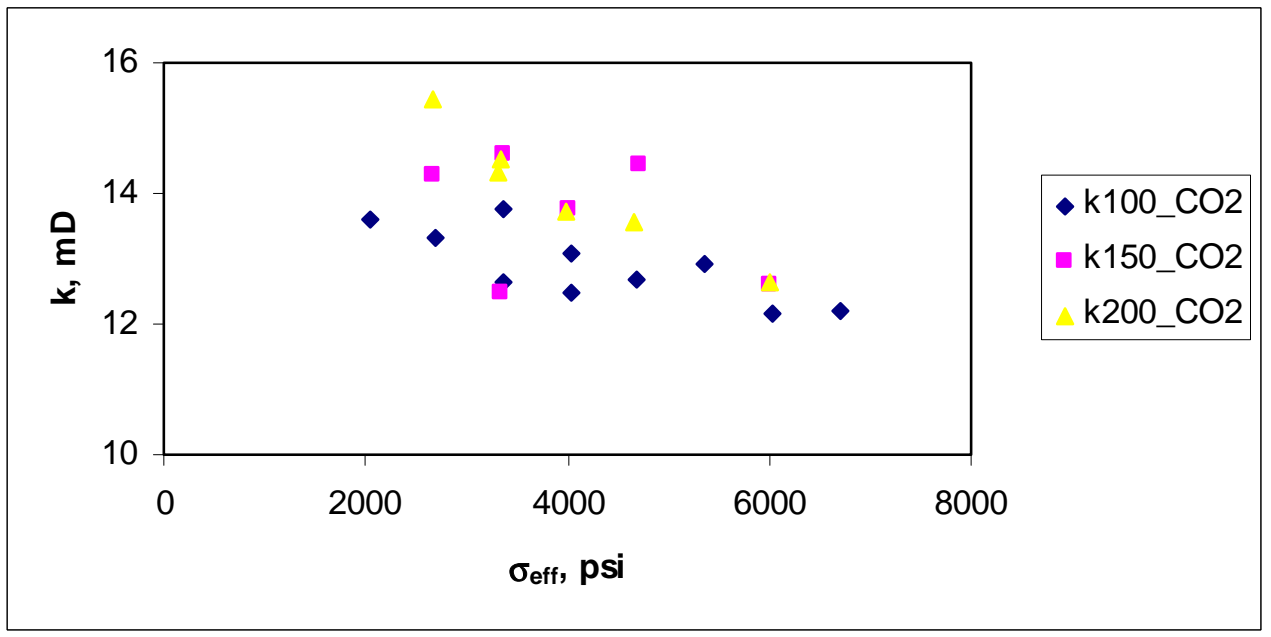

7(b). $\mathrm{k}_{\mathrm{CO} 2}$ under different temperatures

Fig. 6-7. Comparison of temperature effects on permeability in $\mathrm{N}_{2}$ and $\mathrm{CO}_{2}$ flooding. 


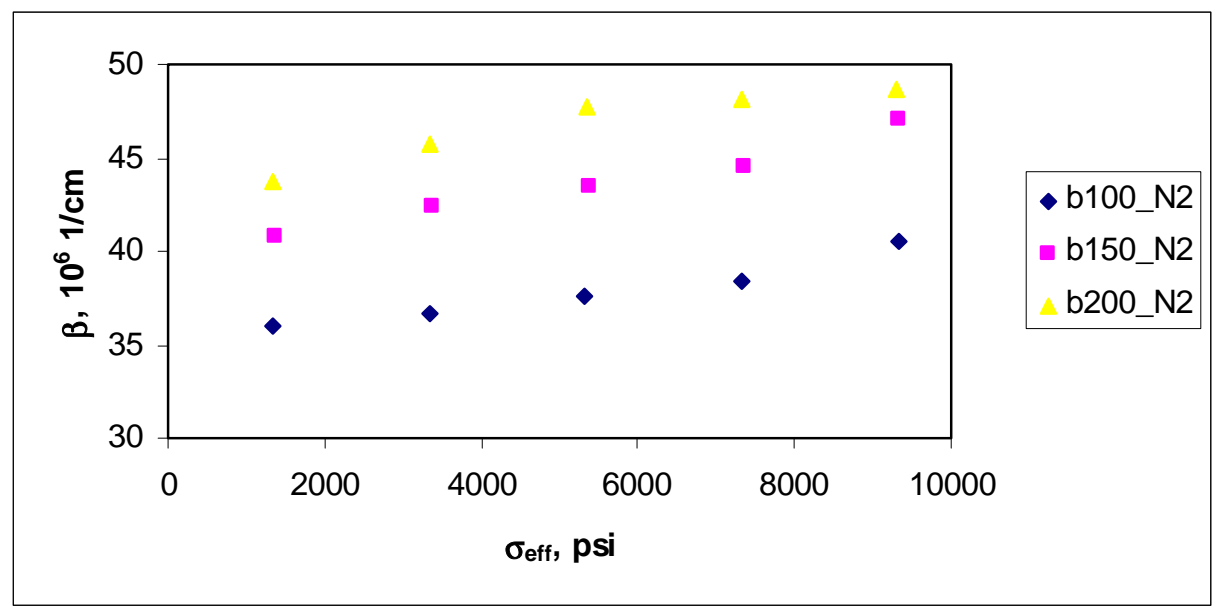

8(a). $\beta_{\mathrm{N} 2}$ under different temperatures

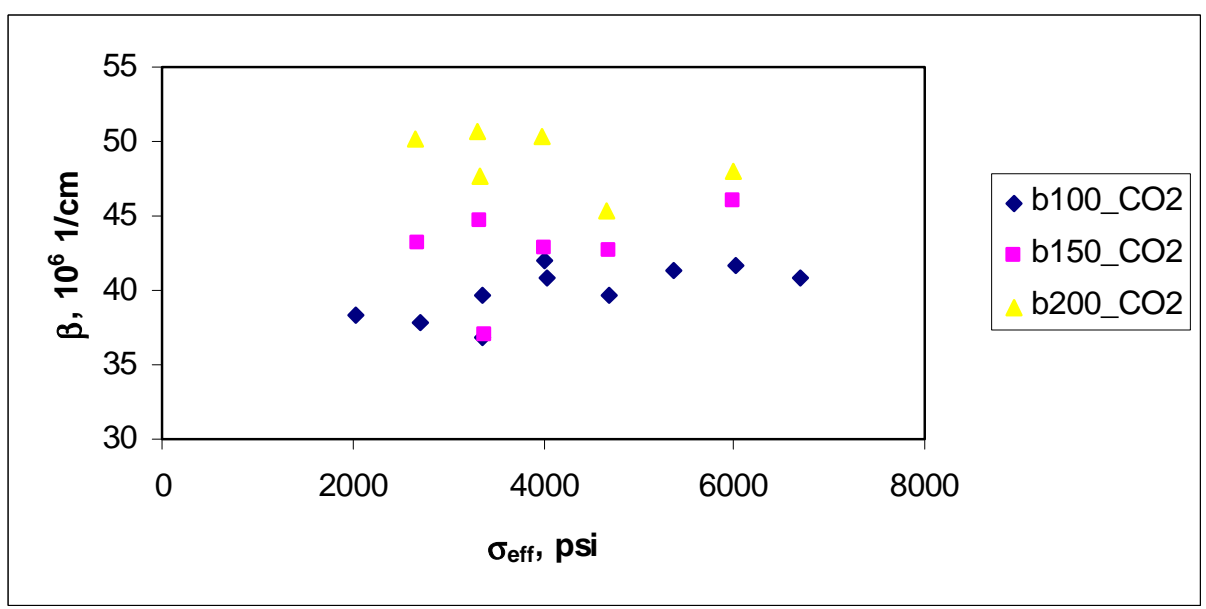

8(b). $\beta_{\mathrm{CO} 2}$ under different temperatures

Fig. 6-8. Comparison of temperature effects on non-Darcy flow coefficient in $\mathrm{N}_{2}$ and $\mathrm{CO}_{2}$ flooding. 


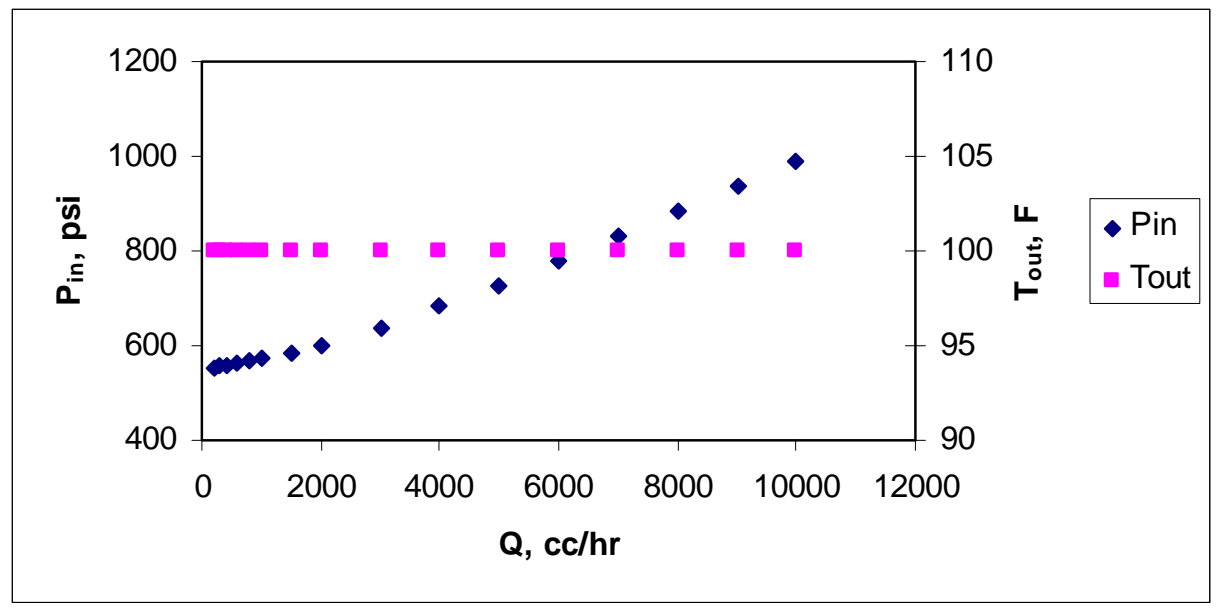

Fig. 6-9. Stable thermal equilibrium in $\mathrm{N}_{2}$ flooding experiments in test $\mathrm{N}_{2} \_100 \_4000 \_4000 \_500$ in IL301: no change of temperature with the increase of flow rate and inlet pressure.

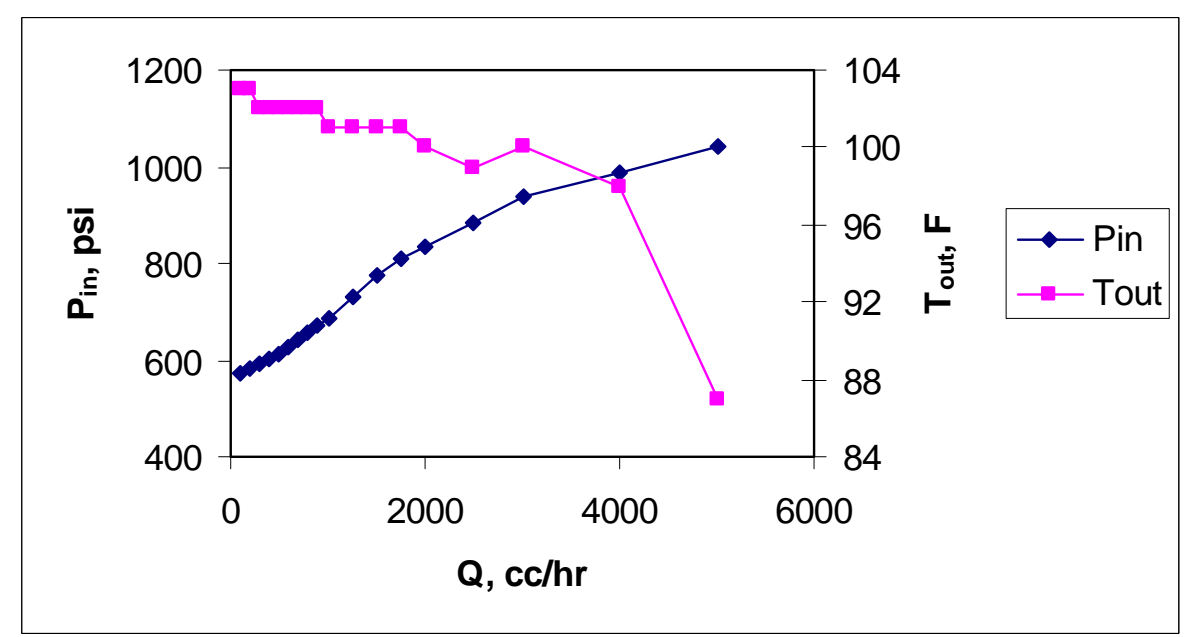

Fig. 6-10

Fig. 6-10. Unstable thermal equilibrium in $\mathrm{CO}_{2}$ flooding experiments in test $\mathrm{CO}_{2}$ 100_4000_2000_500 in IL301: temperature decreases rapidly with increase of flow rate and inlet pressure. 


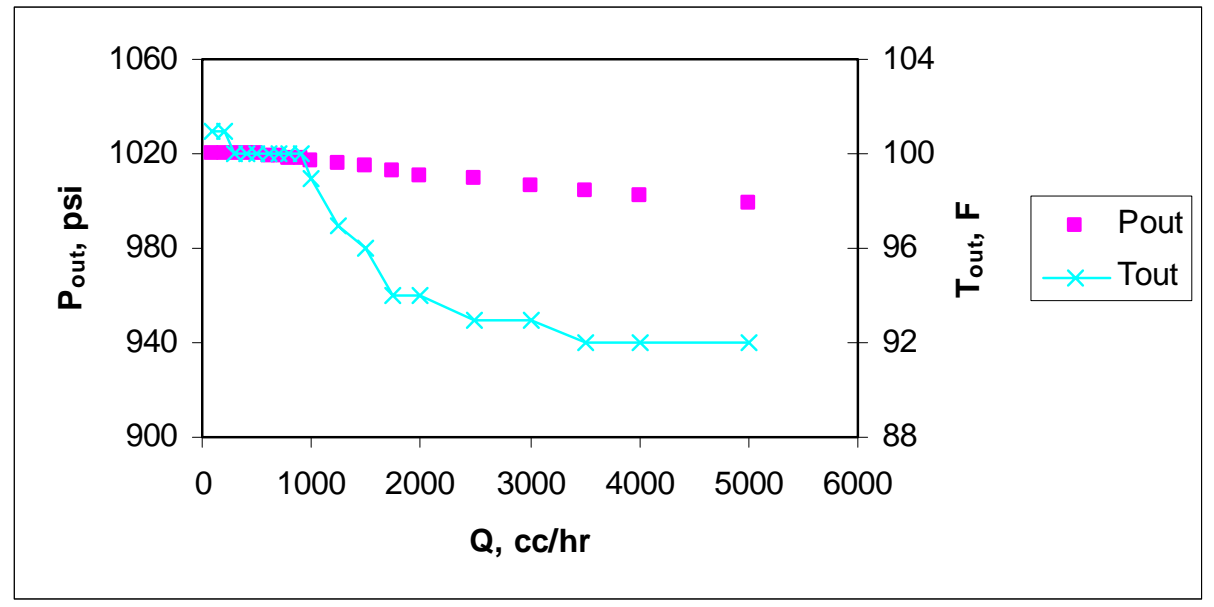

Fig. 6-11. Unstable thermal equilibrium in $\mathrm{CO}_{2}$ flooding experiments in test $\mathrm{CO}_{2}$ 100_6000_3000_1000 in IL301: temperature started decreasing rapidly at relatively low flow rate (outlet pressure was set at about $1000 \mathrm{psi}$ ).

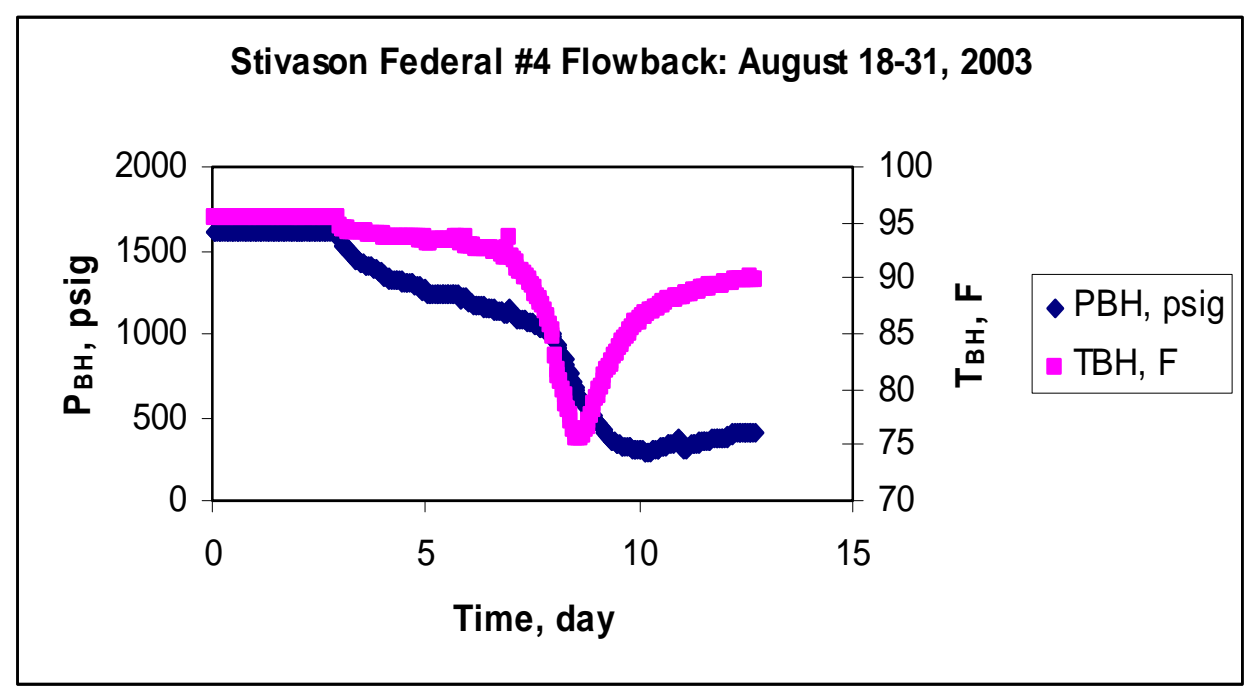

Fig. 6-12. Cooling effect started when bottomhole conditions approached the critical conditions of $\mathrm{CO}_{2}$. Bottomhole pressure did not recover. 